The USGS Groundwater Resources Program

Synoptic Water-Level Measurements of the Upper Floridan Aquifer in Florida and Parts of Georgia, South Carolina, and Alabama, May-June 2010

Data Series 639

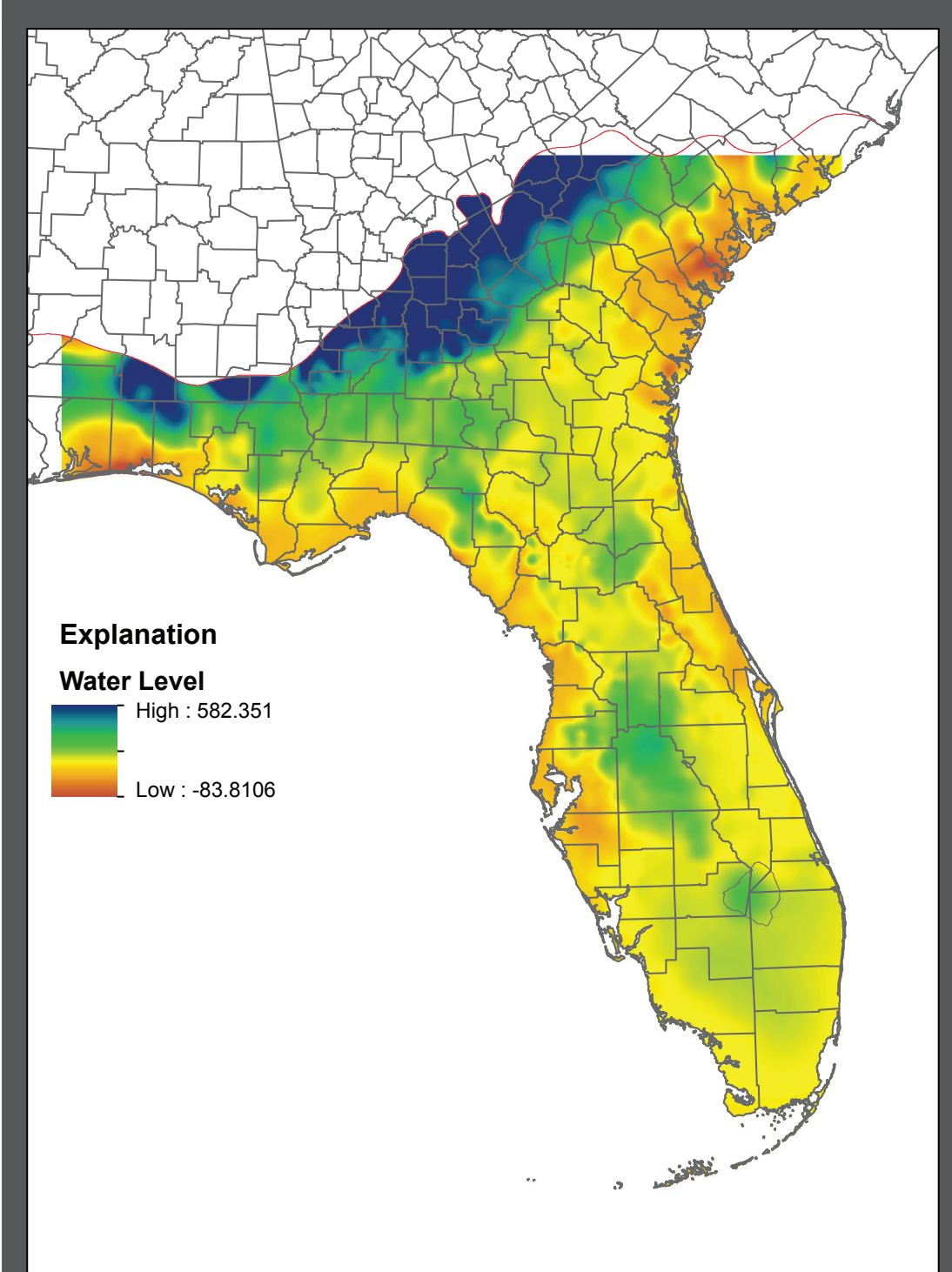




\section{Synoptic Water-Level Measurements of the Upper Floridan Aquifer in Florida and Parts of Georgia, South Carolina, and Alabama, May-June 2010}

By Sandra L. Kinnaman

The USGS Groundwater Resources Program

Data Series 639

U.S. Department of the Interior

U.S. Geological Survey 
U.S. Department of the Interior

KEN SALAZAR, Secretary

\section{U.S. Geological Survey}

Marcia K. McNutt, Director

U.S. Geological Survey, Reston, Virginia: 2012

For more information on the USGS - the Federal source for science about the Earth, its natural and living resources, natural hazards, and the environment, visit http://www.usgs.gov or call 1-888-ASK-USGS

For an overview of USGS information products, including maps, imagery, and publications,

visit http://www.usgs.gov/pubprod

To order this and other USGS information products, visit http://store.usgs.gov

Any use of trade, product, or firm names is for descriptive purposes only and does not imply endorsement by the U.S. Government.

Although this report is in the public domain, permission must be secured from the individual copyright owners to reproduce any copyrighted materials contained within this report.

Suggested citation:

Kinnaman, S.L., 2012, Synoptic Water-Level Measurements of the Upper Floridan Aquifer in Florida and Parts of Georgia, South Carolina, and Alabama, May-June 2010, Data Series 639, 107 p 


\section{Acknowledgments}

Water-level measurements were made by or in cooperation with personnel of the Alachua County Environmental Protection Department, Florida; Florida Geological Survey; Geological Survey of Alabama; Northwest Florida Water Management District; St. Johns River Water Management District; South Carolina Department of Natural Resources; South Carolina Department of Health and Environmental Control; South Florida Water Management District; Southwest Florida Water Management District; Suwannee River Water Management District; Tampa Bay Water, Florida; U.S. Geological Survey Florida Water Science Center; U.S. Geological Survey Georgia Water Science Center; and the U.S. Geological Survey South Carolina Water Science Center. The Georgia Environmental Protection Division Agricultural Permitting Unit and U.S. Department of Agriculture Natural Resources Conservation Service also provided valuable support. 


\section{Contents}

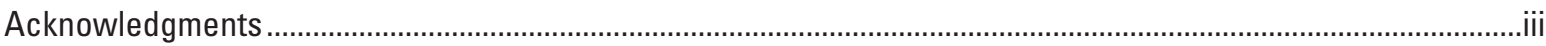

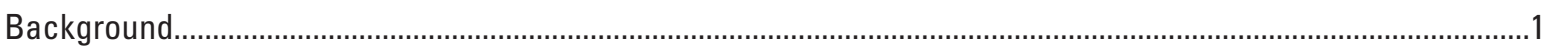

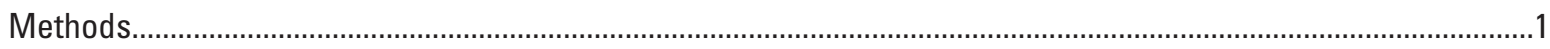

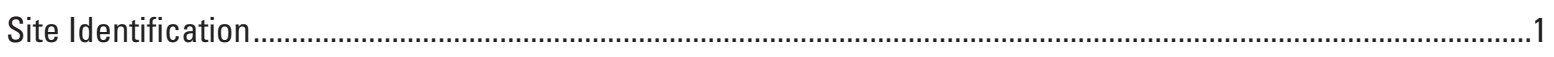

References Cited.

\section{Tables}

Table 1. Water-level measurements in Florida and parts of Georgia, South Carolina, and Alabama,

May-June 2010.

\section{Conversion Factors}

\begin{tabular}{ccc}
\hline Multiply & By & To obtain \\
\hline & Length & \\
\hline foot $(\mathrm{ft})$ & 0.3048 & meter $(\mathrm{m})$ \\
\hline
\end{tabular}

Vertical coordinate information is referenced to the National Geodetic Vertical Datum of 1929 (NGVD 29).

Altitude, as used in this report, refers to distance above the vertical datum.

\section{Abbreviations and Acronyms}

$\begin{array}{ll}\text { ACEPD } & \text { Alachua County Environmental Protection Department } \\ \text { ALWSC } & \text { Alabama Water Science Center } \\ \text { FGS } & \text { Florida Geological Survey } \\ \text { FLWSC } & \text { Florida Water Science Center } \\ \text { GAWSC } & \text { Georgia Water Science Center } \\ \text { GSA } & \text { Geological Survey of Alabama } \\ \text { GWSI } & \text { Groundwater Site-Inventory System } \\ \text { NWFWMD } & \text { Northwest Florida Water Management District } \\ \text { NWIS } & \text { National Water Information System } \\ \text { SCWSC } & \text { South Carolina Water Science Center } \\ \text { SFWMD } & \text { South Florida Water Management District } \\ \text { SRWMD } & \text { Suwannee River Water Management District } \\ \text { USGS } & \text { U.S. Geological Survey }\end{array}$




\title{
Synoptic Water-Level Measurements of the Upper Floridan Aquifer in Florida and Parts of Georgia, South Carolina, and Alabama, May-June 2010
}

\author{
By Sandra L. Kinnaman
}

\section{Background}

Water levels for the Upper Floridan aquifer were measured throughout Florida and in parts of Georgia, South Carolina, and Alabama in May-June 2010.

These measurements were compiled for the U.S. Geological Survey (USGS) Floridan Aquifer System Groundwater Availability Study and conducted as part of the USGS Groundwater Resources Program. Data were collected by personnel from the USGS Florida Water Science Center, Georgia Water Science Center, South Carolina Water Science Center and several state and county agencies in Florida, Georgia, South Carolina, and Alabama using standard techniques. Data collected by USGS personnel are stored in the USGS National Water Information System (NWIS), Groundwater Site-Inventory System (GWSI). Furnished records from cooperators are stored in NWIS/GWSI when possible, but are available from the source agency.

This report consists of synoptic water-level altitudes measured in the Upper Floridan aquifer in Florida and parts of Georgia, South Carolina, and Alabama in May-June 2010. These data were compiled for the regional USGS Floridan Aquifer System Groundwater Availability Study, accessed August 2011, at http://fl.water.usgs.gov/FASWAM/, that was developed as part of the USGS Groundwater Resources Program, Regional Groundwater Studies to improve the understanding of groundwater availability in major aquifers across the Nation, accessed August 2011, at http://water.usgs.gov/ogw/gwrp/ activities/regional.html.

\section{Methods}

This report presents a compilation of the 1,753 water-level measurements collected using standard techniques and methods (Cunningham and Schalk, 2011) by the USGS and several federal, state, and county agencies in Florida, Georgia, South Carolina, and Alabama. The data set consists of 1,266 sites measured in Florida, 428 in Georgia, 45 in South Carolina, and 14 in Alabama (table 1). The data were used to develop a regional potentiometric surface map of the Upper Floridan aquifer in May-June 2010 (Kinnaman and Dixon, 2011). Previous synoptic measurements and regional potentiometric maps of the Upper Floridan aquifer were prepared for May 1980 (Johnston and others, 1981) and May 1985

(Bush and others, 1986) as part of the USGS Floridan Regional Aquifer System Analysis study.

\section{Site Identification}

Sites and corresponding water-level altitudes are identified by either a unique USGS site identification number that has been established in the NWIS or local unique site identifier as provided by the source agency. Completion of the metadata and field verification of the well location and attributes for all of the furnished record was beyond the scope of the study. Therefore, the furnished data are aggregated and documented in this report rather than permanently archived in NWIS. Inquiries about specific measurements should be directed to the source agency that furnished the record.

\section{References Cited}

Bush, P.W., Barr, G.L., Clarke, J.S., and Johnston, R.H., 1986, Potentiometric surface of the Upper Floridan aquifer in Florida and in parts of Georgia, South Carolina, and Alabama, May 1985: U.S. Geological Survey Water-Resources Investigations Report 86-4316, 1 pl.

Cunningham, W.L., and Schalk, C.W., comps., 2011, Groundwater technical procedures of the U.S.

Geological Survey: U.S. Geological Survey Techniques and Methods 1-A1, $151 \mathrm{p}$.

Johnston, R.H., Healy, H.G., and Hayes, L.R., 1981, Potentiometric surface of the Upper Floridan aquifer in Florida and in parts of Georgia, South Carolina, and Alabama, May 1980: U.S. Geological Survey Open-File Report 81-486, 1 pl.

Kinnaman, S.L., and Dixon, J.F., 2011, Potentiometric surface of the Upper Floridan aquifer in Florida and in parts of Georgia, South Carolina, and Alabama, May-June 2010: U.S. Geological Survey Scientific Investigations Map 3182, 1 pl. (Also available at http://pubs.usgs.gov/sim/3182.) 
Table 1. Water-level measurements in Florida and parts of Georgia, South Carolina, and Alabama, May-June 2010

[NGVD 29, National Geodetic Vertical Datum of 1929. Abbreviations for agency database: ACEPD, Alachua County Environmental Protection Department; FGS, Florida Geological Survey; GSA, Geological Survey of Alabama; NWFWMD, Northwest Florida Water Management District; SFWMD, South Florida Water Management District; SRWMD, Suwannee River Water Management District; USGSALWSC, U.S. Geological Survey Alabama Water Science Center; USGS-FLWSC, U.S. Geological Survey Florida Water Science Center; GAWSC, U.S. Geological Survey Georgia Water Science Center; SCWSC, U.S. Geological Survey South Carolina Water Science Center. Abbreviations for Station names: BLVD., boulevard; CO, county; CR, county road; E, east; HS, high school; IFAS, Institute of Food and Agricultural Services; LK, lake; MW, monitoring well; N, north; NE, northeast; NE, northeast; NF, national forest; NW, northwest; PK, park; R., river; RD., road; SE, southeast; SE, southeast; SR, state road; ST., street; TWR, tower; UFA, Upper Floridan aquifer; - - not available; \#, number; “, inches]

\begin{tabular}{|c|c|c|c|c|c|c|c|c|c|c|}
\hline State & $\begin{array}{c}\text { Agency } \\
\text { database }\end{array}$ & Station name & $\begin{array}{c}\text { USGS site } \\
\text { identification } \\
\text { number }\end{array}$ & $\begin{array}{l}\text { Local agency } \\
\text { unique site } \\
\text { identifier }\end{array}$ & County & $\begin{array}{l}\text { Latitude } \\
\text { (degree } \\
\text { minutes } \\
\text { and } \\
\text { seconds) } \\
\text { [DDMMSS] }\end{array}$ & $\begin{array}{l}\text { Longitude } \\
\text { (degree } \\
\text { minutes } \\
\text { and } \\
\text { seconds) } \\
\text { ] [DDMMSS] }\end{array}$ & $\begin{array}{c}\text { Date of } \\
\text { measure- } \\
\text { ment }\end{array}$ & $\begin{array}{c}\text { Water } \\
\text { level } \\
\text { altitude } \\
\text { (feet } \\
\text { above } \\
\text { NGVD 29) }\end{array}$ & Remarks \\
\hline $\mathrm{AL}$ & GSA & V-04 (HOU-1) & 310132085024001 & - & Houston & 310134 & 850238 & $5 / 21 / 2010$ & 112 & \\
\hline $\mathrm{AL}$ & USGS-ALWSC & AA 1-USGS 310105086314401 & 310105086314401 & - & Covington & 310104 & 863145 & $5 / 20 / 2010$ & 164 & \\
\hline $\mathrm{AL}$ & USGS-ALWSC & CC 5-USGS 310221086185201 & 310221086185201 & - & Covington & 310225 & 861854 & $5 / 20 / 2010$ & 216 & \\
\hline $\mathrm{AL}$ & USGS-ALWSC & Mathis & 310017085063401 & - & Houston & 310017 & 850634 & $5 / 21 / 2010$ & 125 & \\
\hline $\mathrm{AL}$ & USGS-ALWSC & Q 77 & 310910086504001 & - & Escambia & 310915 & 865043 & $5 / 20 / 2010$ & 128 & \\
\hline $\mathrm{AL}$ & USGS-ALWSC & Q 9-USGS 310446085451501 & 310446085451501 & - & Geneva & 310442 & 854507 & $5 / 21 / 2010$ & 203 & \\
\hline $\mathrm{AL}$ & USGS-ALWSC & R 1-USGS 310539085232601 & 310539085232601 & - & Houston & 310223 & 852538 & $5 / 21 / 2010$ & 163 & \\
\hline $\mathrm{AL}$ & USGS-ALWSC & S 10-USGS 310153085225901 & 310153085225901 & - & Houston & 310152 & 852257 & $5 / 21 / 2010$ & 138 & \\
\hline $\mathrm{AL}$ & USGS-ALWSC & S 8-USGS 310208085194001 & 310208085194001 & - & Houston & 310218 & 851927 & $5 / 21 / 2010$ & 131 & \\
\hline $\mathrm{AL}$ & USGS-ALWSC & T 10-USGS 310329085145401 & 310329085145401 & - & Houston & 310326 & 851454 & $5 / 21 / 2010$ & 133 & \\
\hline $\mathrm{AL}$ & USGS-ALWSC & T 9-USGS 310143086012701 & 310143086012701 & - & Geneva & 310143 & 860127 & $5 / 20 / 2010$ & 122 & \\
\hline $\mathrm{AL}$ & USGS-ALWSC & U 8-USGS 310325085090301 & 310325085090301 & - & Houston & 310313 & 850903 & $5 / 21 / 2010$ & 144 & \\
\hline AL & USGS-ALWSC & X 4-USGS 305945085535901 & 305945085535901 & - & Geneva & 305940 & 855401 & $5 / 21 / 2010$ & 91 & \\
\hline $\mathrm{AL}$ & USGS-ALWSC & Z 4-USGS 310151086363301 & 310151086363301 & - & Covington & 310150 & 863629 & $5 / 20 / 2010$ & 193 & \\
\hline FL & ACEPD & Alcorn & - & - & Alachua & 294004 & 822732 & $5 / 19 / 2010$ & 45 & \\
\hline FL & ACEPD & Asbell & 292850082323001 & - & Alachua & 292850 & 823230 & $5 / 17 / 2010$ & 44 & \\
\hline FL & ACEPD & Batey & 293133082311201 & - & Alachua & 293133 & 823113 & $5 / 17 / 2010$ & 43 & \\
\hline FL & ACEPD & Bickmeyer & - & - & Alachua & 293614 & 820458 & $5 / 17 / 2010$ & 75 & \\
\hline FL & ACEPD & Bliss & - & - & Alachua & 294819 & 822211 & $5 / 18 / 2010$ & 44 & $\begin{array}{l}\text { SRWMD site iden- } \\
\text { tification number } \\
\text {-081912004 }\end{array}$ \\
\hline FL & ACEPD & Blue Springs Staff Gauge & 02322350 & - & Gilchrist & 294948 & 824059 & $5 / 19 / 2010$ & 25 & \\
\hline FL & ACEPD & Broadway & - & - & Alachua & 295216 & 822310 & $5 / 18 / 2010$ & 45 & \\
\hline FL & ACEPD & Butt & - & - & Gilchrist & 295039 & 824235 & $5 / 17 / 2010$ & 23 & \\
\hline
\end{tabular}


Table 1. Water-level measurements in Florida and parts of Georgia, South Carolina, and Alabama, May-June 2010.—Continued

[NGVD 29, National Geodetic Vertical Datum of 1929. Abbreviations for agency database: ACEPD, Alachua County Environmental Protection Department; FGS, Florida Geological Survey; GSA, Geological Survey of Alabama; NWFWMD, Northwest Florida Water Management District; SFWMD, South Florida Water Management District; SRWMD, Suwannee River Water Management District; USGSALWSC, U.S. Geological Survey Alabama Water Science Center; USGS-FLWSC, U.S. Geological Survey Florida Water Science Center; GAWSC, U.S. Geological Survey Georgia Water Science Center; SCWSC, U.S. Geological Survey South Carolina Water Science Center. Abbreviations for Station names: BLVD., boulevard; CO, county; CR, county road; E, east; HS, high school; IFAS, Institute of Food and Agricultural Services; LK, lake; MW, monitoring well; N, north; NE, northeast; NE, northeast; NF, national forest; NW, northwest; PK, park; R., river; RD., road; SE, southeast; SE, southeast; SR, state road; ST., street; TWR, tower; UFA, Upper Floridan aquifer; - , not available; \#, number; “, inches]

\begin{tabular}{|c|c|c|c|c|c|c|c|c|c|c|}
\hline State & $\begin{array}{c}\text { Agency } \\
\text { database }\end{array}$ & Station name & $\begin{array}{c}\text { USGS site } \\
\text { identification } \\
\text { number }\end{array}$ & $\begin{array}{l}\text { Local agency } \\
\text { unique site } \\
\text { identifier }\end{array}$ & County & $\begin{array}{l}\text { Latitude } \\
\text { (degree } \\
\text { minutes } \\
\text { and } \\
\text { seconds) } \\
\text { [DDMMSS] }\end{array}$ & $\begin{array}{l}\text { Longitude } \\
\text { (degree } \\
\text { minutes } \\
\text { and } \\
\text { seconds) } \\
\text { [DDMMSS] }\end{array}$ & $\begin{array}{c}\text { Date of } \\
\text { measure- } \\
\text { ment }\end{array}$ & $\begin{array}{c}\text { Water } \\
\text { level } \\
\text { altitude } \\
\text { (feet } \\
\text { above } \\
\text { NGVD 29) }\end{array}$ & Remarks \\
\hline FL & ACEPD & $\begin{array}{l}\text { Camp Kulaqua (River Ranch } \\
\text { Well) }\end{array}$ & 295100082350701 & - & Alachua & 295100 & 823507 & $5 / 19 / 2010$ & 33 & \\
\hline FL & ACEPD & Church of God by Faith (G-13) & - & - & Alachua & 294043 & 821523 & $5 / 18 / 2010$ & 57 & \\
\hline FL & ACEPD & City of Waldo (G-34) & 294726082101001 & - & Alachua & 294726 & 821010 & $5 / 2 / 2010$ & 69 & \\
\hline FL & ACEPD & Clark & - & - & Alachua & 294322 & 823055 & $5 / 18 / 2010$ & 37 & \\
\hline FL & ACEPD & Copeland Park (G-5) & - & - & Alachua & 294040 & 821438 & $5 / 21 / 2010$ & 59 & \\
\hline FL & ACEPD & Dollar General & - & - & Alachua & 294612 & 823225 & $5 / 19 / 2010$ & 35 & \\
\hline FL & ACEPD & DOT FL-15 & - & - & Alachua & 294329 & 821621 & $5 / 18 / 2010$ & 55 & \\
\hline FL & ACEPD & DOT FL-28 & - & - & Alachua & 294212 & 821641 & $5 / 18 / 2010$ & 48 & \\
\hline FL & ACEPD & Fahning (Taylor) & - & - & Alachua & 292907 & 820941 & $5 / 17 / 2010$ & 55 & \\
\hline FL & ACEPD & FHP Station (G-26) & 294241082211601 & - & Alachua & 294241 & 822116 & $5 / 18 / 2010$ & 45 & \\
\hline FL & ACEPD & Garrett & - & - & Alachua & 294009 & 822137 & $5 / 19 / 2010$ & 47 & \\
\hline FL & ACEPD & $\begin{array}{l}\text { Gilchrist Co. NE Recycling } \\
\text { Center }\end{array}$ & - & - & Gilchrist & 294641 & 824421 & $5 / 20 / 2010$ & 41 & \\
\hline FL & ACEPD & Gilchrist NE & - & - & Gilchrist & 294831 & 824427 & $5 / 20 / 2010$ & 33 & \\
\hline FL & ACEPD & Gilchrist SE & - & - & Gilchrist & 293816 & 824121 & $5 / 18 / 2010$ & 41 & \\
\hline FL & ACEPD & Ginnie Springs & 295010082420101 & - & Gilchrist & 295010 & 824201 & $5 / 18 / 2010$ & 23 & \\
\hline FL & ACEPD & Gorenberg & - & - & Alachua & 293746 & 822555 & $5 / 17 / 2010$ & 43 & \\
\hline FL & ACEPD & Gresham & - & - & Alachua & 293955 & 823128 & $5 / 18 / 2010$ & 39 & \\
\hline FL & ACEPD & Grimes (Wilkes) & - & - & Alachua & 294336 & 821510 & $5 / 17 / 2010$ & 60 & \\
\hline FL & ACEPD & Hall-Manship & 295246082393101 & - & Columbia & 295246 & 823931 & $5 / 20 / 2010$ & 32 & \\
\hline FL & ACEPD & High Springs Sprayfield & - & - & Alachua & 294737 & 823728 & $5 / 19 / 2010$ & 31 & \\
\hline FL & ACEPD & Hines & 293946082334301 & - & Alachua & 293946 & 823343 & $5 / 18 / 2010$ & 39 & \\
\hline FL & ACEPD & Hodge & - & - & Gilchrist & 294337 & 824103 & $5 / 17 / 2010$ & 33 & \\
\hline FL & ACEPD & Hogle & - & - & Alachua & 294119 & 822925 & $5 / 18 / 2010$ & 42 & \\
\hline
\end{tabular}


Table 1. Water-level measurements in Florida and parts of Georgia, South Carolina, and Alabama, May-June 2010.—Continued

[NGVD 29, National Geodetic Vertical Datum of 1929. Abbreviations for agency database: ACEPD, Alachua County Environmental Protection Department; FGS, Florida Geological Survey; GSA, Geological Survey of Alabama; NWFWMD, Northwest Florida Water Management District; SFWMD, South Florida Water Management District; SRWMD, Suwannee River Water Management District; USGSALWSC, U.S. Geological Survey Alabama Water Science Center; USGS-FLWSC, U.S. Geological Survey Florida Water Science Center; GAWSC, U.S. Geological Survey Georgia Water Science Center; SCWSC, U.S. Geological Survey South Carolina Water Science Center. Abbreviations for Station names: BLVD., boulevard; CO, county; CR, county road; E, east; HS, high school; IFAS, Institute of Food and Agricultural Services; LK, lake; MW, monitoring well; N, north; NE, northeast; NE, northeast; NF, national forest; NW, northwest; PK, park; R., river; RD., road; SE, southeast; SE, southeast; SR, state road; ST., street; TWR, tower; UFA, Upper Floridan aquifer; - , not available; \#, number; “, inches]

\begin{tabular}{|c|c|c|c|c|c|c|c|c|c|c|}
\hline State & $\begin{array}{l}\text { Agency } \\
\text { database }\end{array}$ & Station name & $\begin{array}{c}\text { USGS site } \\
\text { identification } \\
\text { number }\end{array}$ & $\begin{array}{l}\text { Local agency } \\
\text { unique site } \\
\text { identifier }\end{array}$ & County & $\begin{array}{c}\text { Latitude } \\
\text { (degree } \\
\text { minutes } \\
\text { and } \\
\text { seconds) } \\
\text { [DDMMSS] }\end{array}$ & $\begin{array}{l}\text { Longitude } \\
\text { (degree } \\
\text { minutes } \\
\text { and } \\
\text { seconds) } \\
\text { ] [DDMMSS] }\end{array}$ & $\begin{array}{c}\text { Date of } \\
\text { measure- } \\
\text { ment }\end{array}$ & $\begin{array}{c}\text { Water } \\
\text { level } \\
\text { altitude } \\
\text { (feet } \\
\text { above } \\
\text { NGVD 29) }\end{array}$ & Remarks \\
\hline FL & ACEPD & Holliman & - & - & Alachua & 293545 & 822539 & $5 / 17 / 2010$ & 44 & \\
\hline FL & ACEPD & Joan Pocklington & 295259082315701 & - & Alachua & 295259 & 823157 & $5 / 18 / 2010$ & 34 & \\
\hline FL & ACEPD & Jones & 293537082385701 & - & Alachua & 293538 & 823856 & $5 / 17 / 2010$ & 42 & $\begin{array}{l}\text { SRWMD Site iden- } \\
\text { tification number } \\
-101719001\end{array}$ \\
\hline FL & ACEPD & Julie Pocklington & 294359082345001 & - & Alachua & 294359 & 823450 & $5 / 18 / 2010$ & 35 & \\
\hline FL & ACEPD & July & - & - & Columbia & 295455 & 823831 & $5 / 20 / 2010$ & 33 & \\
\hline FL & ACEPD & Karst Environmental & - & - & Gilchrist & 294853 & 824241 & $5 / 17 / 2010$ & 26 & \\
\hline FL & ACEPD & Lander & 294805082355901 & - & Alachua & 294805 & 823559 & $5 / 19 / 2010$ & 34 & \\
\hline FL & ACEPD & LeClaire & 294745082502301 & 一 & Gilchrist & 294745 & 825023 & $5 / 20 / 2010$ & 31 & \\
\hline FL & ACEPD & Loncala North & - & - & Gilchrist & 294821 & 824028 & $5 / 20 / 2010$ & 28 & \\
\hline FL & ACEPD & Loncala Office & 294620082365701 & - & Alachua & 294620 & 823657 & $5 / 18 / 2010$ & 33 & \\
\hline FL & ACEPD & Loncala Ranch & 294422082390801 & - & Alachua & 294422 & 823908 & $5 / 8 / 2010$ & 33 & \\
\hline FL & ACEPD & Loncala South & - & - & Gilchrist & 294419 & 824027 & $5 / 18 / 2010$ & 33 & \\
\hline FL & ACEPD & Majors & - & - & Alachua & 293830 & 822626 & $5 / 17 / 2010$ & 43 & \\
\hline FL & ACEPD & Mcalhaney & - & - & Columbia & 295257 & 823645 & $5 / 20 / 2010$ & 34 & \\
\hline FL & ACEPD & McDilda & - & - & Alachua & 293847 & 823657 & $5 / 17 / 2010$ & 38 & \\
\hline FL & ACEPD & $\begin{array}{l}\text { McGurn Investments } \\
\quad(\text { HRS,G-06) }\end{array}$ & 294005082184901 & - & Alachua & 294005 & 821849 & $5 / 19 / 2010$ & 46 & \\
\hline FL & ACEPD & Milne & - & - & Alachua & 295144 & 823443 & $5 / 19 / 2010$ & 33 & \\
\hline FL & ACEPD & MW 1-F & 294629082181301 & - & Alachua & 294628 & 821808 & $5 / 19 / 2010$ & 58 & \\
\hline FL & ACEPD & MW 2-F & 294454082185601 & 一 & Alachua & 294454 & 821856 & $5 / 19 / 2010$ & 48 & \\
\hline FL & ACEPD & MW 4-F & - & - & Alachua & 294241 & 821822 & $5 / 19 / 2010$ & 32 & \\
\hline FL & ACEPD & NE-2D (G-24) & - & - & Alachua & 294255 & 821437 & $5 / 21 / 2010$ & 63 & \\
\hline FL & ACEPD & Newberry Cemetary & 293813082370201 & - & Alachua & 293813 & 823702 & $5 / 17 / 2010$ & 39 & \\
\hline
\end{tabular}


Table 1. Water-level measurements in Florida and parts of Georgia, South Carolina, and Alabama, May-June 2010.-Continued

[NGVD 29, National Geodetic Vertical Datum of 1929. Abbreviations for agency database: ACEPD, Alachua County Environmental Protection Department; FGS, Florida Geological Survey; GSA, Geological Survey of Alabama; NWFWMD, Northwest Florida Water Management District; SFWMD, South Florida Water Management District; SRWMD, Suwannee River Water Management District; USGSALWSC, U.S. Geological Survey Alabama Water Science Center; USGS-FLWSC, U.S. Geological Survey Florida Water Science Center; GAWSC, U.S. Geological Survey Georgia Water Science Center; SCWSC, U.S. Geological Survey South Carolina Water Science Center. Abbreviations for Station names: BLVD., boulevard; CO, county; CR, county road; E, east; HS, high school; IFAS, Institute of Food and Agricultural Services; LK, lake; MW, monitoring well; N, north; NE, northeast; NE, northeast; NF, national forest; NW, northwest; PK, park; R., river; RD., road; SE, southeast; SE, southeast; SR, state road; ST., street; TWR, tower; UFA, Upper Floridan aquifer; - , not available; \#, number; “, inches]

\begin{tabular}{|c|c|c|c|c|c|c|c|c|c|c|}
\hline State & $\begin{array}{c}\text { Agency } \\
\text { database }\end{array}$ & Station name & $\begin{array}{c}\text { USGS site } \\
\text { identification } \\
\text { number }\end{array}$ & $\begin{array}{l}\text { Local agency } \\
\text { unique site } \\
\text { identifier }\end{array}$ & County & $\begin{array}{l}\text { Latitude } \\
\text { (degree } \\
\text { minutes } \\
\text { and } \\
\text { seconds) } \\
\text { [DDMMSS] }\end{array}$ & $\begin{array}{l}\text { Longitude } \\
\text { (degree } \\
\text { minutes } \\
\text { and } \\
\text { seconds) } \\
\text { [DDMMSS] }\end{array}$ & $\begin{array}{c}\text { Date of } \\
\text { measure- } \\
\text { ment }\end{array}$ & $\begin{array}{c}\text { Water } \\
\text { level } \\
\text { altitude } \\
\text { (feet } \\
\text { above } \\
\text { NGVD 29) }\end{array}$ & Remarks \\
\hline FL & ACEPD & Newmans & 294415082170701 & - & Alachua & 294417 & 821707 & $5 / 18 / 2010$ & 49 & \\
\hline FL & ACEPD & Olson & 294631082060501 & - & Alachua & 294631 & 820605 & $5 / 17 / 2010$ & 79 & \\
\hline FL & ACEPD & Poe Springs & 02322140 & - & Alachua & 294932 & 823856 & $5 / 19 / 2010$ & 27 & \\
\hline FL & ACEPD & Poe Springs Production & 294922082391301 & - & Alachua & 294922 & 823913 & $5 / 19 / 2010$ & 27 & \\
\hline FL & ACEPD & PUGH (Kerr) & - & - & Alachua & 294746 & 823250 & $5 / 19 / 2010$ & 34 & \\
\hline FL & ACEPD & PW8 & - & - & Alachua & 294028 & 823610 & $5 / 18 / 2010$ & 36 & \\
\hline FL & ACEPD & Raulerson & - & - & Gilchrist & 294739 & 824439 & $5 / 20 / 2010$ & 31 & \\
\hline FL & ACEPD & Rauseo & - & - & Gilchrist & 295334 & 824811 & $5 / 20 / 2010$ & 55 & \\
\hline FL & ACEPD & Stavely & - & - & Gilchrist & 294334 & 824353 & $5 / 17 / 2010$ & 42 & \\
\hline FL & ACEPD & Sulek & 295453082421001 & - & Columbia & 295453 & 824210 & $5 / 20 / 2010$ & 31 & \\
\hline FL & ACEPD & Tipton & 293310082304001 & - & Alachua & 293310 & 823040 & $5 / 17 / 2010$ & 42 & \\
\hline FL & ACEPD & TREEO Center & - & - & Alachua & 293713 & 822454 & $5 / 17 / 2010$ & 46 & \\
\hline FL & ACEPD & Truluck & 293730082335401 & - & Alachua & 293730 & 823354 & $5 / 18 / 2010$ & 39 & $\begin{array}{l}\text { SRWMD Site iden- } \\
\text { tification number } \\
-101713003\end{array}$ \\
\hline FL & ACEPD & United Methodist Church & - & - & Alachua & 293919 & 821924 & $5 / 19 / 2010$ & 46 & \\
\hline FL & ACEPD & VISA \#1 & 294119082290401 & - & Alachua & 294120 & 822902 & $5 / 18 / 2010$ & 42 & \\
\hline FL & ACEPD & VISA \#2 & 294028082245301 & - & Alachua & 294028 & 822453 & $5 / 19 / 2010$ & 46 & \\
\hline FL & ACEPD & VISA \#3 & 294011082260401 & - & Alachua & 294028 & 822447 & $5 / 19 / 2010$ & 46 & \\
\hline FL & ACEPD & VISA \#4 & - & - & Alachua & 293803 & 822326 & $5 / 17 / 2010$ & 49 & \\
\hline FL & ACEPD & Waters (G-17) & - & - & Alachua & 294746 & 821839 & $5 / 18 / 2010$ & 56 & \\
\hline FL & ACEPD & Weiss & 295346082460101 & - & Gilchrist & 295346 & 824601 & $5 / 20 / 2010$ & 16 & \\
\hline FL & ACEPD & Willis & 295515082260101 & - & Alachua & 295515 & 822601 & $5 / 18 / 2010$ & 48 & \\
\hline FL & ACEPD & Wood & 294148082315501 & - & Alachua & 294148 & 823155 & $5 / 18 / 2010$ & 44 & \\
\hline
\end{tabular}


Table 1. Water-level measurements in Florida and parts of Georgia, South Carolina, and Alabama, May-June 2010.—Continued

[NGVD 29, National Geodetic Vertical Datum of 1929. Abbreviations for agency database: ACEPD, Alachua County Environmental Protection Department; FGS, Florida Geological Survey; GSA, Geological Survey of Alabama; NWFWMD, Northwest Florida Water Management District; SFWMD, South Florida Water Management District; SRWMD, Suwannee River Water Management District; USGSALWSC, U.S. Geological Survey Alabama Water Science Center; USGS-FLWSC, U.S. Geological Survey Florida Water Science Center; GAWSC, U.S. Geological Survey Georgia Water Science Center; SCWSC, U.S. Geological Survey South Carolina Water Science Center. Abbreviations for Station names: BLVD., boulevard; CO, county; CR, county road; E, east; HS, high school; IFAS, Institute of Food and Agricultural Services; LK, lake; MW, monitoring well; N, north; NE, northeast; NE, northeast; NF, national forest; NW, northwest; PK, park; R., river; RD., road; SE, southeast; SE, southeast; SR, state road; ST., street; TWR, tower; UFA, Upper Floridan aquifer; - , not available; \#, number; “, inches]

\begin{tabular}{|c|c|c|c|c|c|c|c|c|c|c|}
\hline State & $\begin{array}{c}\text { Agency } \\
\text { database }\end{array}$ & Station name & $\begin{array}{c}\text { USGS site } \\
\text { identification } \\
\text { number }\end{array}$ & $\begin{array}{l}\text { Local agency } \\
\text { unique site } \\
\text { identifier }\end{array}$ & County & $\begin{array}{l}\text { Latitude } \\
\text { (degree } \\
\text { minutes } \\
\text { and } \\
\text { seconds) } \\
\text { [DDMMSS] }\end{array}$ & $\begin{array}{l}\text { Longitude } \\
\text { (degree } \\
\text { minutes } \\
\text { and } \\
\text { seconds) } \\
\text { [DDMMSS] }\end{array}$ & $\begin{array}{c}\text { Date of } \\
\text { measure- } \\
\text { ment }\end{array}$ & $\begin{array}{c}\text { Water } \\
\text { level } \\
\text { altitude } \\
\text { (feet } \\
\text { above } \\
\text { NGVD 29) }\end{array}$ & Remarks \\
\hline FL & ACEPD & $\begin{array}{l}\text { WUFT Transmission Tower } \\
\text { (G-30) }\end{array}$ & 294235082234101 & - & Alachua & 294235 & 822341 & $5 / 18 / 2010$ & 46 & \\
\hline FL & ACEPD & Yates & - & - & Alachua & 294443 & 823135 & $5 / 18 / 2010$ & 36 & \\
\hline FL & FGS & BYRD DAIRY MW \#11 & - & 51319007 & Lafayette & 300231 & 830322 & $5 / 19 / 2010$ & 22 & $\begin{array}{l}\text { SRWMD Site iden- } \\
\text { tification number } \\
-051319007\end{array}$ \\
\hline FL & FGS & Clemons, Betty & - & 61313006 & Lafayette & 295748 & 825824 & $5 / 26 / 2010$ & 27 & $\begin{array}{l}\text { SRWMD Site iden- } \\
\text { tification number } \\
-061313006\end{array}$ \\
\hline FL & FGS & DOF-LIVE OAK W/C & - & 21316007 & Suwannee & 301838 & 830113 & $5 / 20 / 2010$ & 39 & $\begin{array}{l}\text { SRWMD Site iden- } \\
\text { tification number } \\
-021316007\end{array}$ \\
\hline FL & FGS & EDWARDS CE 1 & - & 31331002 & Suwannee & 301117 & 830307 & $5 / 18 / 2010$ & 32 & $\begin{array}{l}\text { SRWMD Site iden- } \\
\text { tification number } \\
-031331002\end{array}$ \\
\hline FL & FGS & Gwinn, Terry Donnell & - & 41317001 & Suwannee & 300818 & 830242 & $5 / 17 / 2010$ & 28 & $\begin{array}{l}\text { SRWMD Site iden- } \\
\text { tification number } \\
-041317001\end{array}$ \\
\hline FL & FGS & Hicks, Doris L. & - & 41533003 & Suwannee & 300526 & 824909 & $5 / 28 / 2010$ & 33 & $\begin{array}{l}\text { SRWMD Site iden- } \\
\text { tification number } \\
-041533003\end{array}$ \\
\hline FL & FGS & $\begin{array}{l}\text { ICHETUCKNEE STATE } \\
\text { PARK MW \#7 }\end{array}$ & - & 61514002 & Suwannee & 295744 & 824706 & $5 / 19 / 2010$ & 17 & $\begin{array}{l}\text { SRWMD Site iden- } \\
\text { tification number } \\
-061514002\end{array}$ \\
\hline FL & FGS & IFAS MW\#3 JOHN TAYLOR & - & 31101006 & Suwannee & 301512 & 831034 & $5 / 25 / 2010$ & 37 & $\begin{array}{l}\text { SRWMD Site iden- } \\
\text { tification number } \\
-031101006\end{array}$ \\
\hline
\end{tabular}


Table 1. Water-level measurements in Florida and parts of Georgia, South Carolina, and Alabama, May-June 2010.—Continued

[NGVD 29, National Geodetic Vertical Datum of 1929. Abbreviations for agency database: ACEPD, Alachua County Environmental Protection Department; FGS, Florida Geological Survey; GSA, Geological Survey of Alabama; NWFWMD, Northwest Florida Water Management District; SFWMD, South Florida Water Management District; SRWMD, Suwannee River Water Management District; USGSALWSC, U.S. Geological Survey Alabama Water Science Center; USGS-FLWSC, U.S. Geological Survey Florida Water Science Center; GAWSC, U.S. Geological Survey Georgia Water Science Center; SCWSC, U.S. Geological Survey South Carolina Water Science Center. Abbreviations for Station names: BLVD., boulevard; CO, county; CR, county road; E, east; HS, high school; IFAS, Institute of Food and Agricultural Services; LK, lake; MW, monitoring well; N, north; NE, northeast; NE, northeast; NF, national forest; NW, northwest; PK, park; R., river; RD., road; SE, southeast; SE, southeast; SR, state road; ST., street; TWR, tower; UFA, Upper Floridan aquifer; - , not available; \#, number; “, inches]

\begin{tabular}{|c|c|c|c|c|c|c|c|c|c|c|}
\hline State & $\begin{array}{l}\text { Agency } \\
\text { database }\end{array}$ & Station name & $\begin{array}{c}\text { USGS site } \\
\text { identification } \\
\text { number }\end{array}$ & $\begin{array}{l}\text { Local agency } \\
\text { unique site } \\
\text { identifier }\end{array}$ & County & $\begin{array}{l}\text { Latitude } \\
\text { (degree } \\
\text { minutes } \\
\text { and } \\
\text { seconds) } \\
\text { [DDMMSS] }\end{array}$ & $\begin{array}{l}\text { Longitude } \\
\text { (degree } \\
\text { minutes } \\
\text { and } \\
\text { seconds) } \\
\text { [DDMMSS] }\end{array}$ & $\begin{array}{c}\text { Date of } \\
\text { measure- } \\
\text { ment }\end{array}$ & $\begin{array}{c}\text { Water } \\
\text { level } \\
\text { altitude } \\
\text { (feet } \\
\text { above } \\
\text { NGVD 29) }\end{array}$ & Remarks \\
\hline FL & FGS & $\begin{array}{l}\text { LAFAYETTE CO COMM- } \\
\quad \text { VISA } 2\end{array}$ & - & 51203002 & Lafayette & 300444 & 830601 & $6 / 4 / 2010$ & 22 & $\begin{array}{l}\text { SRWMD Site iden- } \\
\text { tification number } \\
-051203002\end{array}$ \\
\hline FL & FGS & McCray, Mary Alice & - & 61201001 & Lafayette & 295934 & 830502 & $5 / 24 / 2010$ & 69 & $\begin{array}{l}\text { SRWMD Site iden- } \\
\text { tification number } \\
\text {-061201001 }\end{array}$ \\
\hline FL & FGS & PLEASANT HILL CHURCH & - & 61324005 & Suwannee & 300722 & 825843 & $5 / 26 / 2010$ & 29 & $\begin{array}{l}\text { SRWMD Site iden- } \\
\text { tification number } \\
-041324005\end{array}$ \\
\hline FL & FGS & WET FARMS-ROBERTS \#2 & - & 51419010 & Suwannee & 300143 & 825658 & $5 / 26 / 2010$ & 24 & $\begin{array}{l}\text { SRWMD Site iden- } \\
\text { tification number } \\
-051419010\end{array}$ \\
\hline FL & NWFWMD & AL LAWSON & - & 5363 & Okaloosa & 305306 & 862422 & $6 / 9 / 2010$ & 155 & \\
\hline FL & NWFWMD & ALEX MEYER & - & 5962 & Washington & 302940 & 854317 & $6 / 8 / 2010$ & 44 & \\
\hline FL & NWFWMD & ALTHA EAST \#1 & 303415085074001 & 3775 & Calhoun & 303415 & 850740 & $6 / 9 / 2010$ & 57 & \\
\hline FL & NWFWMD & APALACHICOLA \#5 & 294310085013802 & 8351 & Franklin & 294310 & 850135 & $6 / 9 / 2010$ & 7 & \\
\hline FL & NWFWMD & ARGONAUT STREET 02 & 301210085505902 & 679 & Bay & 301211 & 855060 & $6 / 7 / 2010$ & -6 & \\
\hline FL & NWFWMD & ARGYLE TOWER & 304334086032401 & 4754 & Walton & 304337 & 860327 & $6 / 7 / 2010$ & 80 & \\
\hline FL & NWFWMD & BAKER / MCCOLLUM & 301403085112801 & 748 & Calhoun & 301434 & 851130 & $6 / 9 / 2010$ & 31 & \\
\hline FL & NWFWMD & BAY CO-HARDERS PARK \#2 & - & 785 & Bay & 301436 & 853029 & $6 / 7 / 2010$ & 28 & \\
\hline FL & NWFWMD & $\begin{array}{l}\text { BAY CO STEELFIELD LF } \\
\text { OFFICE }\end{array}$ & - & 5976 & Bay & 302132 & 855504 & $6 / 8 / 2010$ & 33 & \\
\hline FL & NWFWMD & BILLY MILLER & - & 4822 & Holmes & 304455 & 855157 & $6 / 7 / 2010$ & 55 & \\
\hline FL & NWFWMD & BONIFAY \#2 & 304710085410801 & 5033 & Holmes & 304710 & 854107 & $6 / 9 / 2010$ & 99 & \\
\hline FL & NWFWMD & BUCK REGISTER & 303319084355701 & 3653 & Gadsden & 303321 & 843555 & $6 / 7 / 2010$ & 59 & \\
\hline FL & NWFWMD & BUCKEYE CELLULOSE 41 & 295732084430701 & 215 & Franklin & 295733 & 844308 & $6 / 9 / 2010$ & 14 & \\
\hline
\end{tabular}


Table 1. Water-level measurements in Florida and parts of Georgia, South Carolina, and Alabama, May-June 2010.—Continued

[NGVD 29, National Geodetic Vertical Datum of 1929. Abbreviations for agency database: ACEPD, Alachua County Environmental Protection Department; FGS, Florida Geological Survey; GSA, Geological Survey of Alabama; NWFWMD, Northwest Florida Water Management District; SFWMD, South Florida Water Management District; SRWMD, Suwannee River Water Management District; USGSALWSC, U.S. Geological Survey Alabama Water Science Center; USGS-FLWSC, U.S. Geological Survey Florida Water Science Center; GAWSC, U.S. Geological Survey Georgia Water Science Center; SCWSC, U.S. Geological Survey South Carolina Water Science Center. Abbreviations for Station names: BLVD., boulevard; CO, county; CR, county road; E, east; HS, high school; IFAS, Institute of Food and Agricultural Services; LK, lake; MW, monitoring well; N, north; NE, northeast; NE, northeast; NF, national forest; NW, northwest; PK, park; R., river; RD., road; SE, southeast; SE, southeast; SR, state road; ST., street; TWR, tower; UFA, Upper Floridan aquifer; - - not available; \#, number; “, inches]

\begin{tabular}{|c|c|c|c|c|c|c|c|c|c|c|}
\hline State & $\begin{array}{l}\text { Agency } \\
\text { database }\end{array}$ & Station name & $\begin{array}{c}\text { USGS site } \\
\text { identification } \\
\text { number }\end{array}$ & $\begin{array}{l}\text { Local agency } \\
\text { unique site } \\
\text { identifier }\end{array}$ & County & $\begin{array}{c}\text { Latitude } \\
\text { (degree } \\
\text { minutes } \\
\text { and } \\
\text { seconds) } \\
\text { [DDMMSS] }\end{array}$ & $\begin{array}{l}\text { Longitude } \\
\text { (degree } \\
\text { minutes } \\
\text { and } \\
\text { seconds) } \\
\text { ] [DDMMSS] }\end{array}$ & $\begin{array}{c}\text { Date of } \\
\text { measure- } \\
\text { ment }\end{array}$ & $\begin{array}{c}\text { Water } \\
\text { level } \\
\text { altitude } \\
\text { (feet } \\
\text { above } \\
\text { NGVD 29) }\end{array}$ & Remarks \\
\hline FL & NWFWMD & $\begin{array}{l}\text { C. DONAHUE DEEP / NI- } \\
\text { TRATE POT. MAP }\end{array}$ & 301844084173502 & 978 & Leon & 301840 & 841738 & $6 / 23 / 2010$ & 7 & \\
\hline FL & NWFWMD & C. HOUSEHOLDER & - & 5254 & Holmes & 305101 & 855426 & $6 / 7 / 2010$ & 68 & \\
\hline FL & NWFWMD & CAMP G JOHNSTON \#10 & 295507084311901 & 182 & Franklin & 295507 & 843118 & $6 / 8 / 2010$ & 10 & \\
\hline FL & NWFWMD & CARRABELLE ICE PLANT & 295046084394301 & 111 & Franklin & 295046 & 843940 & $6 / 9 / 2010$ & 4 & \\
\hline FL & NWFWMD & $\begin{array}{l}\text { CITY OF TALLAHASSEE / } \\
\text { LAFAYETTE PARK }\end{array}$ & 302710084163001 & 2691 & Leon & 302711 & 841630 & $6 / 8 / 2010$ & 25 & \\
\hline FL & NWFWMD & $\begin{array}{l}\text { COLONIAL PINES TRAILER } \\
\text { PARK }\end{array}$ & - & 2051 & Santa Rosa & 302447 & 864833 & $6 / 8 / 2010$ & -49 & \\
\hline FL & NWFWMD & COMPASS LAKE TOWER & 303701085231301 & 4183 & Jackson & 303700 & 852312 & $6 / 9 / 2010$ & 100 & \\
\hline FL & NWFWMD & COTTONDALE \#3 & 304746085223201 & 5062 & Jackson & 304747 & 852232 & $6 / 7 / 2010$ & 116 & \\
\hline FL & NWFWMD & COUCH CONSTRUCTION & 302327085260701 & 1637 & Bay & 302327 & 852604 & $6 / 7 / 2010$ & 92 & \\
\hline FL & NWFWMD & COY STAFFORD & - & 5691 & Holmes & 305838 & 855922 & $6 / 7 / 2010$ & 123 & \\
\hline FL & NWFWMD & CRESTVIEW \#4 & 304518086334001 & 4855 & Okaloosa & 304519 & 863339 & $6 / 7 / 2010$ & 21 & \\
\hline FL & NWFWMD & CRESTVIEW \#6 & - & 7171 & Okaloosa & 304240 & 863502 & $6 / 7 / 2010$ & 11 & \\
\hline FL & NWFWMD & CRESTVIEW EAST REMOTE & - & 8758 & Okaloosa & 304225 & 862718 & $6 / 9 / 2010$ & 49 & \\
\hline FL & NWFWMD & $\begin{array}{l}\text { CRESTVIEW WEST RE- } \\
\text { MOTE }\end{array}$ & - & 8759 & Okaloosa & 304404 & 863941 & $6 / 9 / 2010$ & 13 & \\
\hline FL & NWFWMD & CYTEC UPPER FLRD & 303413087063802 & 3771 & Santa Rosa & 303414 & 870638 & $6 / 10 / 2010$ & 5 & \\
\hline FL & NWFWMD & DITTY JC-1 (JK-28) & 305905085063401 & 5718 & Jackson & 305904 & 850634 & $6 / 7 / 2010$ & 118 & \\
\hline FL & NWFWMD & DUREN & - & 5963 & Washington & 302655 & 854212 & $5 / 27 / 2010$ & 43 & \\
\hline FL & NWFWMD & DWU \#5 (503'-747') & - & 1796 & Okaloosa & 302403 & 863032 & $6 / 9 / 2010$ & -49 & \\
\hline FL & NWFWMD & $\begin{array}{l}\text { DWU-4048 INDIAN RIVER } \\
\text { TRAIL MO\#1 }\end{array}$ & - & 9137 & Okaloosa & 302418 & 862743 & $6 / 9 / 2010$ & -43 & \\
\hline FL & NWFWMD & EAFB-ALAQUA TOWER & 304219086140201 & 5802 & Walton & 304219 & 861404 & $6 / 7 / 2010$ & 95 & \\
\hline
\end{tabular}


Table 1. Water-level measurements in Florida and parts of Georgia, South Carolina, and Alabama, May-June 2010.—Continued

[NGVD 29, National Geodetic Vertical Datum of 1929. Abbreviations for agency database: ACEPD, Alachua County Environmental Protection Department; FGS, Florida Geological Survey; GSA, Geological Survey of Alabama; NWFWMD, Northwest Florida Water Management District; SFWMD, South Florida Water Management District; SRWMD, Suwannee River Water Management District; USGSALWSC, U.S. Geological Survey Alabama Water Science Center; USGS-FLWSC, U.S. Geological Survey Florida Water Science Center; GAWSC, U.S. Geological Survey Georgia Water Science Center; SCWSC, U.S. Geological Survey South Carolina Water Science Center. Abbreviations for Station names: BLVD., boulevard; CO, county; CR, county road; E, east; HS, high school; IFAS, Institute of Food and Agricultural Services; LK, lake; MW, monitoring well; N, north; NE, northeast; NE, northeast; NF, national forest; NW, northwest; PK, park; R., river; RD., road; SE, southeast; SE, southeast; SR, state road; ST., street; TWR, tower; UFA, Upper Floridan aquifer; —, not available; \#, number; “, inches]

\begin{tabular}{|c|c|c|c|c|c|c|c|c|c|c|}
\hline State & $\begin{array}{l}\text { Agency } \\
\text { database }\end{array}$ & Station name & $\begin{array}{c}\text { USGS site } \\
\text { identification } \\
\text { number }\end{array}$ & $\begin{array}{l}\text { Local agency } \\
\text { unique site } \\
\text { identifier }\end{array}$ & County & $\begin{array}{l}\text { Latitude } \\
\text { (degree } \\
\text { minutes } \\
\text { and } \\
\text { seconds) } \\
\text { [DDMMSS] }\end{array}$ & $\begin{array}{l}\text { Longitude } \\
\text { (degree } \\
\text { minutes } \\
\text { and } \\
\text { seconds) } \\
\text { [DDMMSS] }\end{array}$ & $\begin{array}{c}\text { Date of } \\
\text { measure- } \\
\text { ment }\end{array}$ & $\begin{array}{c}\text { Water } \\
\text { level } \\
\text { altitude } \\
\text { (feet } \\
\text { above } \\
\text { NGVD 29) }\end{array}$ & Remarks \\
\hline FL & NWFWMD & EAFB \#61 HARDSTAND & 302857086310701 & 2995 & Okaloosa & 302847 & 863114 & $6 / 8 / 2010$ & -54 & \\
\hline FL & NWFWMD & $\begin{array}{l}\text { EAFB FLD \#1 WELL \#2 } \\
\text { BLDG\#1030/USGS \#19 }\end{array}$ & 304044086211601 & 4553 & Walton & 304046 & 862115 & $6 / 7 / 2010$ & 62 & \\
\hline FL & NWFWMD & $\begin{array}{l}\text { EAFB FLD \#4 WELL \#2 } \\
\text { BLDG\#4204 }\end{array}$ & 303021086351601 & 3209 & Okaloosa & 303022 & 863516 & $6 / 8 / 2010$ & -35 & \\
\hline FL & NWFWMD & EAFB FLD \#5 WELL \#2 & 303512086375101 & 3923 & Okaloosa & 303515 & 863750 & $6 / 7 / 2010$ & -11 & \\
\hline FL & NWFWMD & EAFB FLD\#2 BLDG 2102 & 303431086264501 & 3815 & Okaloosa & 303435 & 862642 & $6 / 7 / 2010$ & -29 & \\
\hline FL & NWFWMD & EAFB FLD\#3 96 CEG/CEVCE & 303849086314101 & 4368 & Okaloosa & 303851 & 863139 & $6 / 7 / 2010$ & 9 & \\
\hline FL & NWFWMD & EAFB HOUSING \#16A & - & 8127 & Okaloosa & 302726 & 863347 & $6 / 8 / 2010$ & -67 & \\
\hline FL & NWFWMD & EAFB METTS TOWER & 303314086453702 & 3642 & Okaloosa & 303315 & 864535 & $6 / 7 / 2010$ & -7 & \\
\hline FL & NWFWMD & EAFB NCO \#71 & - & 6016 & Okaloosa & 302318 & 863129 & $6 / 7 / 2010$ & -47 & \\
\hline FL & NWFWMD & EAFB NR CAMP RUCKER & 302843086184001 & 2993 & Walton & 302854 & 861834 & $6 / 8 / 2010$ & -2 & \\
\hline FL & NWFWMD & EAFB POSTIL POINT & 302857086285201 & 2994 & Okaloosa & 302858 & 862854 & $6 / 8 / 2010$ & -54 & \\
\hline FL & NWFWMD & EAFB R52N ROAD \#200 & - & 3280 & Walton & 303442 & 861941 & $6 / 7 / 2010$ & 10 & \\
\hline FL & NWFWMD & EAFB RANGE 63 \#31 & - & 3797 & Walton & 303422 & 861257 & $6 / 7 / 2010$ & 25 & \\
\hline FL & NWFWMD & EAFB SAN BLAS \#107 & 294032085204501 & 4 & Gulf & 294034 & 852047 & $6 / 10 / 2010$ & 5 & \\
\hline FL & NWFWMD & EAFB SITE C-62 & 303954086133801 & 4490 & Walton & 303927 & 861411 & $6 / 7 / 2010$ & 73 & \\
\hline FL & NWFWMD & EAFB WHITE POINT \#3 & - & 2696 & Okaloosa & 302713 & 862513 & $6 / 8 / 2010$ & -37 & \\
\hline FL & NWFWMD & $\begin{array}{l}\text { EAST MILTON \#3/MILLERS } \\
\text { BLUFF RD. }\end{array}$ & - & 4393 & Santa Rosa & 303901 & 865237 & $6 / 8 / 2010$ & -1 & \\
\hline FL & NWFWMD & ED ROGERS \#2 & - & 2992 & Washington & 302856 & 853951 & $6 / 8 / 2010$ & 48 & \\
\hline FL & NWFWMD & EDDIE BARNES & - & 1524 & Bay & 302349 & 853216 & $6 / 7 / 2010$ & 54 & \\
\hline FL & NWFWMD & ELTON CRUTCHFIELD & - & 5547 & Holmes & 305624 & 854839 & $6 / 7 / 2010$ & 89 & \\
\hline FL & NWFWMD & ERIK REPPEN & - & 1760 & Bay & 302358 & 854149 & $6 / 7 / 2010$ & 45 & \\
\hline
\end{tabular}


Table 1. Water-level measurements in Florida and parts of Georgia, South Carolina, and Alabama, May-June 2010.—Continued

[NGVD 29, National Geodetic Vertical Datum of 1929. Abbreviations for agency database: ACEPD, Alachua County Environmental Protection Department; FGS, Florida Geological Survey; GSA, Geological Survey of Alabama; NWFWMD, Northwest Florida Water Management District; SFWMD, South Florida Water Management District; SRWMD, Suwannee River Water Management District; USGSALWSC, U.S. Geological Survey Alabama Water Science Center; USGS-FLWSC, U.S. Geological Survey Florida Water Science Center; GAWSC, U.S. Geological Survey Georgia Water Science Center; SCWSC, U.S. Geological Survey South Carolina Water Science Center. Abbreviations for Station names: BLVD., boulevard; CO, county; CR, county road; E, east; HS, high school; IFAS, Institute of Food and Agricultural Services; LK, lake; MW, monitoring well; N, north; NE, northeast; NE, northeast; NF, national forest; NW, northwest; PK, park; R., river; RD., road; SE, southeast; SE, southeast; SR, state road; ST., street; TWR, tower; UFA, Upper Floridan aquifer; - - not available; \#, number; “, inches]

\begin{tabular}{|c|c|c|c|c|c|c|c|c|c|c|}
\hline State & $\begin{array}{l}\text { Agency } \\
\text { database }\end{array}$ & Station name & $\begin{array}{c}\text { USGS site } \\
\text { identification } \\
\text { number }\end{array}$ & $\begin{array}{l}\text { Local agency } \\
\text { unique site } \\
\text { identifier }\end{array}$ & County & $\begin{array}{l}\text { Latitude } \\
\text { (degree } \\
\text { minutes } \\
\text { and } \\
\text { seconds) } \\
\text { [DDMMSS] }\end{array}$ & $\begin{array}{l}\text { Longitude } \\
\text { (degree } \\
\text { minutes } \\
\text { and } \\
\text { seconds) } \\
\text { [DDMMSS] }\end{array}$ & $\begin{array}{c}\text { Date of } \\
\text { measure- } \\
\text { ment }\end{array}$ & $\begin{array}{c}\text { Water } \\
\text { level } \\
\text { altitude } \\
\text { (feet } \\
\text { above } \\
\text { NGVD 29) }\end{array}$ & Remarks \\
\hline FL & NWFWMD & $\begin{array}{l}\text { ESTIFFANULGA WORK } \\
\text { CENTER }\end{array}$ & 301708085010301 & 884 & Liberty & 301717 & 850102 & $6 / 7 / 2010$ & 46 & \\
\hline FL & NWFWMD & EXXON SUPPLY & 300703085160401 & 380 & Gulf & 300704 & 851604 & $6 / 10 / 2010$ & 28 & \\
\hline FL & NWFWMD & FAF \#65/AKA USGS 40 & 303251085595701 & 3588 & Walton & 303251 & 855958 & $6 / 10 / 2010$ & 15 & \\
\hline FL & NWFWMD & FAF \#72A & - & 8643 & Walton & 303215 & 855806 & $6 / 10 / 2010$ & 13 & \\
\hline FL & NWFWMD & FAF \#77 & 303157085570601 & 3441 & Walton & 303155 & 855707 & $6 / 10 / 2010$ & 17 & \\
\hline FL & NWFWMD & FAF \#79 & - & 3502 & Walton & 303220 & 855619 & $6 / 10 / 2010$ & 16 & \\
\hline FL & NWFWMD & FAF \#93 & - & 3921 & Walton & 303503 & 855930 & $6 / 10 / 2010$ & 21 & \\
\hline FL & NWFWMD & FAF \#98 & 303417085573101 & 3780 & Walton & 303422 & 855730 & $6 / 10 / 2010$ & 21 & \\
\hline FL & NWFWMD & FANNIN AIRPORT/S675 & 301250085412801 & 697 & Bay & 301251 & 854128 & $6 / 7 / 2010$ & -4 & \\
\hline FL & NWFWMD & FREEPORT \#1 & - & 6036 & Walton & 302931 & 860816 & $6 / 10 / 2010$ & 7 & \\
\hline FL & NWFWMD & $\begin{array}{l}\text { FREEPORT BRUCE MONI- } \\
\text { TOR }\end{array}$ & - & 8505 & Walton & 302834 & 855742 & $6 / 10 / 2010$ & 19 & \\
\hline FL & NWFWMD & $\begin{array}{l}\text { FREEPORT HIGH \#1/OWL'S } \\
\text { HEAD \#5/FAF \#8 }\end{array}$ & - & 3540 & Walton & 303236 & 860648 & $6 / 10 / 2010$ & 8 & \\
\hline FL & NWFWMD & $\begin{array}{l}\text { FREEPORT ICE PLANT/ } \\
\text { USGS WALTON \#8 }\end{array}$ & - & 9054 & Walton & 302949 & 860760 & $6 / 10 / 2010$ & 9 & \\
\hline FL & NWFWMD & $\begin{array}{l}\text { FREEPORT PORTLAND } \\
\text { TEST }\end{array}$ & - & 8762 & Walton & 303043 & 861316 & $6 / 10 / 2010$ & 8 & \\
\hline FL & NWFWMD & GEOGHAGAN & 305110086164801 & 5261 & Walton & 305112 & 861646 & $6 / 9 / 2010$ & 176 & \\
\hline FL & NWFWMD & HOLT \#1 & - & 4704 & Okaloosa & 304258 & 864436 & $6 / 7 / 2010$ & 9 & \\
\hline FL & NWFWMD & IFAS \#4 & - & 3413 & Jefferson & 303148 & 835511 & $6 / 8 / 2010$ & 48 & \\
\hline FL & NWFWMD & INDIAN PASS CAMPGRND & 294102085134801 & 11 & Gulf & 294104 & 851320 & $6 / 10 / 2010$ & 3 & \\
\hline FL & NWFWMD & $\begin{array}{l}\text { INTERNATIONAL PAPER @ } \\
\text { CYPRESS }\end{array}$ & 304413085064401 & 4795 & Jackson & 304409 & 850635 & $6 / 2 / 2010$ & 96 & \\
\hline FL & NWFWMD & J.H. RAWLS & - & 1093 & Bay & 301951 & 855127 & $6 / 7 / 2010$ & 42 & \\
\hline
\end{tabular}


Table 1. Water-level measurements in Florida and parts of Georgia, South Carolina, and Alabama, May-June 2010.—Continued

[NGVD 29, National Geodetic Vertical Datum of 1929. Abbreviations for agency database: ACEPD, Alachua County Environmental Protection Department; FGS, Florida Geological Survey; GSA, Geological Survey of Alabama; NWFWMD, Northwest Florida Water Management District; SFWMD, South Florida Water Management District; SRWMD, Suwannee River Water Management District; USGSALWSC, U.S. Geological Survey Alabama Water Science Center; USGS-FLWSC, U.S. Geological Survey Florida Water Science Center; GAWSC, U.S. Geological Survey Georgia Water Science Center; SCWSC, U.S. Geological Survey South Carolina Water Science Center. Abbreviations for Station names: BLVD., boulevard; CO, county; CR, county road; E, east; HS, high school; IFAS, Institute of Food and Agricultural Services; LK, lake; MW, monitoring well; N, north; NE, northeast; NE, northeast; NF, national forest; NW, northwest; PK, park; R., river; RD., road; SE, southeast; SE, southeast; SR, state road; ST., street; TWR, tower; UFA, Upper Floridan aquifer; —, not available; \#, number; “, inches]

\begin{tabular}{|c|c|c|c|c|c|c|c|c|c|c|}
\hline State & $\begin{array}{l}\text { Agency } \\
\text { database }\end{array}$ & Station name & $\begin{array}{c}\text { USGS site } \\
\text { identification } \\
\text { number }\end{array}$ & $\begin{array}{l}\text { Local agency } \\
\text { unique site } \\
\text { identifier }\end{array}$ & County & $\begin{array}{l}\text { Latitude } \\
\text { (degree } \\
\text { minutes } \\
\text { and } \\
\text { seconds) } \\
\text { [DDMMSS] }\end{array}$ & $\begin{array}{l}\text { Longitude } \\
\text { (degree } \\
\text { minutes } \\
\text { and } \\
\text { seconds) } \\
\text { [DDMMSS] }\end{array}$ & $\begin{array}{c}\text { Date of } \\
\text { measure- } \\
\text { ment }\end{array}$ & $\begin{array}{c}\text { Water } \\
\text { level } \\
\text { altitude } \\
\text { (feet } \\
\text { above } \\
\text { NGVD 29) }\end{array}$ & Remarks \\
\hline FL & NWFWMD & J.M. THOMPSON & 303053086075301 & 3293 & Walton & 303056 & 860752 & $6 / 10 / 2010$ & 9 & \\
\hline FL & NWFWMD & JEMCO WELL & - & 9595 & Walton & 304410 & 860920 & $6 / 7 / 2010$ & 120 & \\
\hline FL & NWFWMD & JOHN WALTON & - & 3680 & Washington & 303332 & 854526 & $6 / 8 / 2010$ & 42 & \\
\hline FL & NWFWMD & $\begin{array}{l}\text { LARRY PLANK FLORIDAN } \\
\text { PRODUCTION \#2 }\end{array}$ & - & 2397 & Santa Rosa & 302610 & 865439 & $6 / 8 / 2010$ & -34 & \\
\hline FL & NWFWMD & LAUREL HILL TOWER & 305615086284801 & 5543 & Okaloosa & 305614 & 862846 & $6 / 9 / 2010$ & 181 & \\
\hline FL & NWFWMD & MARY ESTHER \#2 & 302442086394001 & 2035 & Okaloosa & 302442 & 863941 & $6 / 8 / 2010$ & -83 & \\
\hline FL & NWFWMD & MCCULLOCH \#1 & 294337084532401 & 29 & Franklin & 294339 & 845324 & $6 / 9 / 2010$ & 6 & \\
\hline FL & NWFWMD & MIDWAY \#1 TEST & - & 1888 & Santa Rosa & 302418 & 865215 & $6 / 8 / 2010$ & -38 & \\
\hline FL & NWFWMD & $\begin{array}{l}\text { MONSANTO \#2/SHALLOW } \\
\text { MONITOR }\end{array}$ & 303541087144402 & 4006 & Escambia & 303542 & 871444 & $6 / 10 / 2010$ & 35 & \\
\hline FL & NWFWMD & MOODY TOWER & 302926085503001 & 3082 & Washington & 302926 & 855029 & 6/7/2010 & 25 & \\
\hline FL & NWFWMD & $\begin{array}{l}\text { NEWPORT RECREATION/ } \\
\text { NITRATE POT. MAP }\end{array}$ & 301156084103501 & 671 & Wakulla & 301157 & 841035 & $6 / 8 / 2010$ & 6 & \\
\hline FL & NWFWMD & $\begin{array}{l}\text { NW BAY CTY-SITE \#1 UFA } \\
\text { MONITOR }\end{array}$ & - & 9638 & Bay & 302624 & 854904 & $6 / 1 / 2010$ & 40 & \\
\hline FL & NWFWMD & $\begin{array}{l}\text { NW BAY CTY-SITE \#2 UFA } \\
\text { MONITOR }\end{array}$ & - & 9748 & Bay & 302329 & 854927 & $6 / 1 / 2010$ & 39 & \\
\hline FL & NWFWMD & $\begin{array}{l}\text { NW BAY CTY-SITE \#3 UFA } \\
\text { MONITOR \#1 }\end{array}$ & - & 9941 & Bay & 302429 & 854547 & $6 / 21 / 2010$ & 42 & \\
\hline FL & NWFWMD & $\begin{array}{l}\text { NWFWMD JEFF SANDERS } \\
\text { RD. TH-4 }\end{array}$ & 295638084362301 & 9442 & Franklin & 295637 & 843623 & $6 / 9 / 2010$ & 11 & \\
\hline FL & NWFWMD & $\begin{array}{l}\text { NWFWMD TRACTOR RD. } \\
\text { TH-2 }\end{array}$ & 295923084390701 & 9092 & Franklin & 295924 & 843907 & $6 / 9 / 2010$ & 12 & \\
\hline FL & NWFWMD & $\begin{array}{l}\text { NWFWMD-2 EGG AMBI- } \\
\text { ENT \#3 }\end{array}$ & 305351085013903 & 5408 & Jackson & 305355 & 850140 & $6 / 7 / 2010$ & 98 & \\
\hline
\end{tabular}


Table 1. Water-level measurements in Florida and parts of Georgia, South Carolina, and Alabama, May-June 2010.—Continued

[NGVD 29, National Geodetic Vertical Datum of 1929. Abbreviations for agency database: ACEPD, Alachua County Environmental Protection Department; FGS, Florida Geological Survey; GSA, Geological Survey of Alabama; NWFWMD, Northwest Florida Water Management District; SFWMD, South Florida Water Management District; SRWMD, Suwannee River Water Management District; USGSALWSC, U.S. Geological Survey Alabama Water Science Center; USGS-FLWSC, U.S. Geological Survey Florida Water Science Center; GAWSC, U.S. Geological Survey Georgia Water Science Center; SCWSC, U.S. Geological Survey South Carolina Water Science Center. Abbreviations for Station names: BLVD., boulevard; CO, county; CR, county road; E, east; HS, high school; IFAS, Institute of Food and Agricultural Services; LK, lake; MW, monitoring well; N, north; NE, northeast; NE, northeast; NF, national forest; NW, northwest; PK, park; R., river; RD., road; SE, southeast; SE, southeast; SR, state road; ST., street; TWR, tower; UFA, Upper Floridan aquifer; - - not available; \#, number; “, inches]

\begin{tabular}{|c|c|c|c|c|c|c|c|c|c|c|}
\hline State & $\begin{array}{l}\text { Agency } \\
\text { database }\end{array}$ & Station name & $\begin{array}{c}\text { USGS site } \\
\text { identification } \\
\text { number }\end{array}$ & $\begin{array}{l}\text { Local agency } \\
\text { unique site } \\
\text { identifier }\end{array}$ & County & $\begin{array}{l}\text { Latitude } \\
\text { (degree } \\
\text { minutes } \\
\text { and } \\
\text { seconds) } \\
\text { [DDMMSS] }\end{array}$ & $\begin{array}{l}\text { Longitude } \\
\text { (degree } \\
\text { minutes } \\
\text { and } \\
\text { seconds) } \\
\text { [DDMMSS] }\end{array}$ & $\begin{array}{l}\text { Date of } \\
\text { measure- } \\
\text { ment }\end{array}$ & $\begin{array}{c}\text { Water } \\
\text { level } \\
\text { altitude } \\
\text { (feet } \\
\text { above } \\
\text { NGVD 29) }\end{array}$ & Remarks \\
\hline FL & NWFWMD & $\begin{array}{l}\text { NWFWMD-BLOUNTSTOWN } \\
\text { FLORIDAN }\end{array}$ & 302655085041101 & 2624 & Calhoun & 302701 & 850413 & $5 / 19 / 2010$ & 68 & \\
\hline FL & NWFWMD & $\begin{array}{l}\text { NWFWMD-BRADFORD } \\
\text { BROOK DEEP }\end{array}$ & 302522084224001 & 2137 & Leon & 302522 & 842239 & $6 / 8 / 2010$ & 22 & \\
\hline FL & NWFWMD & $\begin{array}{l}\text { NWFWMD-ESTO FLORI- } \\
\text { DAN }\end{array}$ & - & 5732 & Holmes & 305917 & 853825 & $6 / 7 / 2010$ & 161 & \\
\hline FL & NWFWMD & $\begin{array}{l}\text { NWFWMD-FGS-FLORIDAN } \\
\quad \# 2\end{array}$ & 303109084275405 & 3342 & Gadsden & 303107 & 842749 & $6 / 8 / 2010$ & 47 & \\
\hline FL & NWFWMD & $\begin{array}{l}\text { NWFWMD-GEORGE'S } 40 \\
\text { FLORIDAN }\end{array}$ & - & 5958 & Bay & 302551 & 853635 & 6/9/2010 & 39 & \\
\hline FL & NWFWMD & $\begin{array}{l}\text { NWFWMD-GREEN POINT } \\
\text { FLORIDAN }\end{array}$ & 294702084495301 & 56 & Franklin & 294703 & 844951 & $6 / 9 / 2010$ & 10 & \\
\hline FL & NWFWMD & NWFWMD-HIGHWAY 77 & - & 5954 & Washington & 302731 & 854047 & $6 / 8 / 2010$ & 45 & \\
\hline FL & NWFWMD & $\begin{array}{l}\text { NWFWMD-HWY } 71 \text { NORTH } \\
\text { OF MALONE }\end{array}$ & 305822085095701 & 5671 & Jackson & 305827 & 851003 & $6 / 7 / 2010$ & 126 & \\
\hline FL & NWFWMD & $\begin{array}{l}\text { NWFWMD-JACKSON STILL } \\
\text { FLORDAN }\end{array}$ & 305359086122602 & 5417 & Walton & 305360 & 861225 & $6 / 2 / 2010$ & 196 & \\
\hline FL & NWFWMD & NWFWMD-NITRATE \#5 & 302037084082701 & 7498 & Leon & 302038 & 840827 & $6 / 8 / 2010$ & 21 & \\
\hline FL & NWFWMD & $\begin{array}{l}\text { NWFWMD-PITTMAN VISA/ } \\
\text { S661 }\end{array}$ & 305113085043601 & 5266 & Jackson & 305114 & 850437 & $6 / 16 / 2010$ & 86 & \\
\hline FL & NWFWMD & $\begin{array}{l}\text { NWFWMD-PORT ST JOE } \\
\text { FLORIDAN }\end{array}$ & 294850085171601 & 70 & Gulf & 294847 & 851717 & $6 / 10 / 2010$ & 4 & \\
\hline FL & NWFWMD & $\begin{array}{l}\text { NWFWMD-PORTER POND } \\
\text { EAST }\end{array}$ & - & 5960 & Washington & 303036 & 853111 & $6 / 8 / 2010$ & 52 & \\
\hline FL & NWFWMD & NWFWMD-POWER LINE & - & 5953 & Washington & 302619 & 853846 & $6 / 1 / 2010$ & 41 & \\
\hline FL & NWFWMD & $\begin{array}{l}\text { NWFWMD-PT. WASH. } \\
\text { FLORIDAN APT }\end{array}$ & - & 1062 & Walton & 301937 & 860401 & 6/9/2010 & 20 & \\
\hline
\end{tabular}


Table 1. Water-level measurements in Florida and parts of Georgia, South Carolina, and Alabama, May-June 2010.—Continued

[NGVD 29, National Geodetic Vertical Datum of 1929. Abbreviations for agency database: ACEPD, Alachua County Environmental Protection Department; FGS, Florida Geological Survey; GSA, Geological Survey of Alabama; NWFWMD, Northwest Florida Water Management District; SFWMD, South Florida Water Management District; SRWMD, Suwannee River Water Management District; USGSALWSC, U.S. Geological Survey Alabama Water Science Center; USGS-FLWSC, U.S. Geological Survey Florida Water Science Center; GAWSC, U.S. Geological Survey Georgia Water Science Center; SCWSC, U.S. Geological Survey South Carolina Water Science Center. Abbreviations for Station names: BLVD., boulevard; CO, county; CR, county road; E, east; HS, high school; IFAS, Institute of Food and Agricultural Services; LK, lake; MW, monitoring well; N, north; NE, northeast; NE, northeast; NF, national forest; NW, northwest; PK, park; R., river; RD., road; SE, southeast; SE, southeast; SR, state road; ST., street; TWR, tower; UFA, Upper Floridan aquifer; —, not available; \#, number; “, inches]

\begin{tabular}{|c|c|c|c|c|c|c|c|c|c|c|}
\hline State & $\begin{array}{l}\text { Agency } \\
\text { database }\end{array}$ & Station name & $\begin{array}{c}\text { USGS site } \\
\text { identification } \\
\text { number }\end{array}$ & $\begin{array}{l}\text { Local agency } \\
\text { unique site } \\
\text { identifier }\end{array}$ & County & $\begin{array}{l}\text { Latitude } \\
\text { (degree } \\
\text { minutes } \\
\text { and } \\
\text { seconds) } \\
\text { [DDMMSS] }\end{array}$ & $\begin{array}{l}\text { Longitude } \\
\text { (degree } \\
\text { minutes } \\
\text { and } \\
\text { seconds) } \\
\text { [DDMMSS] }\end{array}$ & $\begin{array}{c}\text { Date of } \\
\text { measure- } \\
\text { ment }\end{array}$ & $\begin{array}{c}\text { Water } \\
\text { level } \\
\text { altitude } \\
\text { (feet } \\
\text { above } \\
\text { NGVD 29) }\end{array}$ & Remarks \\
\hline FL & NWFWMD & $\begin{array}{l}\text { NWFWMD-SAND HILL UP- } \\
\text { PER FLORIDAN }\end{array}$ & 305712086403401 & - & Okaloosa & 305712 & 864034 & $6 / 9 / 2010$ & 127 & \\
\hline FL & NWFWMD & $\begin{array}{l}\text { NWFWMD-SEAGROVE } \\
\text { DEEP FLORIDAN }\end{array}$ & - & 7751 & Walton & 302112 & 861319 & $6 / 9 / 2010$ & 5 & \\
\hline FL & NWFWMD & $\begin{array}{l}\text { NWFWMD-SEAGROVE } \\
\text { SHALLOW FLORIDAN }\end{array}$ & - & 7687 & Walton & 302112 & 861320 & $6 / 9 / 2010$ & 12 & \\
\hline FL & NWFWMD & NWFWMD-TIGER POINT & - & 7686 & Santa Rosa & 302315 & 870324 & $6 / 8 / 2010$ & -17 & \\
\hline FL & NWFWMD & $\begin{array}{l}\text { NWFWMD-WEST HEWETT } \\
\text { FLORIDAN }\end{array}$ & - & 1376 & Walton & 302224 & 861717 & $6 / 7 / 2010$ & -9 & \\
\hline FL & NWFWMD & OCWS-BAKER MONITOR & - & 8350 & Okaloosa & 305116 & 863902 & $6 / 9 / 2010$ & 81 & \\
\hline FL & NWFWMD & OCWS-DORCAS MONITOR & - & 8353 & Okaloosa & 304650 & 862517 & $6 / 8 / 2010$ & 110 & \\
\hline FL & NWFWMD & OCWS-ISL-1 (MONITOR) & - & 1696 & Okaloosa & 302346 & 863509 & $6 / 8 / 2010$ & -64 & \\
\hline FL & NWFWMD & OKALOOSA ASPHALT & 302525085175701 & 2145 & Calhoun & 302525 & 851755 & $6 / 9 / 2010$ & 96 & \\
\hline FL & NWFWMD & $\begin{array}{l}\text { OKALOOSA SCHOOL } \\
\text { BOARD/S673 }\end{array}$ & 302419086362601 & 1894 & Okaloosa & 302422 & 863625 & $6 / 15 / 2010$ & -71 & \\
\hline FL & NWFWMD & OLD COWFORD & 302637085543301 & 2534 & Walton & 302637 & 855434 & $6 / 10 / 2010$ & 19 & \\
\hline FL & NWFWMD & OWL'S HEAD \#1/FAF \#2A & 303426086054001 & 3806 & Walton & 303418 & 860536 & $6 / 10 / 2010$ & 16 & \\
\hline FL & NWFWMD & $\begin{array}{l}\text { PAXTON WELCOME CEN- } \\
\text { TER }\end{array}$ & 305804086180901 & 5656 & Walton & 305806 & 861808 & $6 / 9 / 2010$ & 215 & \\
\hline FL & NWFWMD & PINNACLE PORT \#1R & - & 9194 & Bay & 301558 & 855908 & $6 / 8 / 2010$ & 2 & \\
\hline FL & NWFWMD & PLACID OIL-SUPPLY & 300813084555701 & 413 & Liberty & 300828 & 845553 & $6 / 7 / 2010$ & 53 & \\
\hline FL & NWFWMD & $\begin{array}{l}\text { PLEASANT VALLEY } \\
\text { CHURCH }\end{array}$ & - & 8907 & Walton & 303945 & 855611 & $6 / 10 / 2010$ & 33 & \\
\hline $\mathrm{FL}$ & NWFWMD & $\begin{array}{l}\text { POINT WASHINGTON/MC- } \\
\text { GEE }\end{array}$ & 302221086065201 & 1371 & Walton & 302222 & 860653 & $6 / 9 / 2010$ & 10 & \\
\hline FL & NWFWMD & PONCE DE LEON \#2 & - & 4731 & Holmes & 304321 & 855707 & $6 / 7 / 2010$ & 62 & \\
\hline
\end{tabular}


Table 1. Water-level measurements in Florida and parts of Georgia, South Carolina, and Alabama, May-June 2010.—Continued

[NGVD 29, National Geodetic Vertical Datum of 1929. Abbreviations for agency database: ACEPD, Alachua County Environmental Protection Department; FGS, Florida Geological Survey; GSA, Geological Survey of Alabama; NWFWMD, Northwest Florida Water Management District; SFWMD, South Florida Water Management District; SRWMD, Suwannee River Water Management District; USGSALWSC, U.S. Geological Survey Alabama Water Science Center; USGS-FLWSC, U.S. Geological Survey Florida Water Science Center; GAWSC, U.S. Geological Survey Georgia Water Science Center; SCWSC, U.S. Geological Survey South Carolina Water Science Center. Abbreviations for Station names: BLVD., boulevard; CO, county; CR, county road; E, east; HS, high school; IFAS, Institute of Food and Agricultural Services; LK, lake; MW, monitoring well; N, north; NE, northeast; NE, northeast; NF, national forest; NW, northwest; PK, park; R., river; RD., road; SE, southeast; SE, southeast; SR, state road; ST., street; TWR, tower; UFA, Upper Floridan aquifer; - - not available; \#, number; “, inches]

\begin{tabular}{|c|c|c|c|c|c|c|c|c|c|c|}
\hline State & $\begin{array}{c}\text { Agency } \\
\text { database }\end{array}$ & Station name & $\begin{array}{c}\text { USGS site } \\
\text { identification } \\
\text { number }\end{array}$ & $\begin{array}{l}\text { Local agency } \\
\text { unique site } \\
\text { identifier }\end{array}$ & County & $\begin{array}{l}\text { Latitude } \\
\text { (degree } \\
\text { minutes } \\
\text { and } \\
\text { seconds) } \\
\text { [DDMMSS] }\end{array}$ & $\begin{array}{l}\text { Longitude } \\
\text { (degree } \\
\text { minutes } \\
\text { and } \\
\text { seconds) } \\
\text { [DDMMSS] }\end{array}$ & $\begin{array}{c}\text { Date of } \\
\text { measure- } \\
\text { ment }\end{array}$ & $\begin{array}{c}\text { Water } \\
\text { level } \\
\text { altitude } \\
\text { (feet } \\
\text { above } \\
\text { NGVD 29) }\end{array}$ & Remarks \\
\hline FL & NWFWMD & PORT ST JOE \#3 & 294936085175001 & 95 & Gulf & 294936 & 851750 & $6 / 9 / 2010$ & 3 & \\
\hline FL & NWFWMD & PROSPERITY TOWER & 305119085561901 & 5271 & Holmes & 305120 & 855618 & $6 / 7 / 2010$ & 81 & \\
\hline FL & NWFWMD & PURDOM \#4 & 301008084123801 & 554 & Wakulla & 301009 & 841238 & $6 / 8 / 2010$ & 4 & \\
\hline FL & NWFWMD & QUINCY \#3 & 303550084345001 & 4026 & Gadsden & 303555 & 843447 & $6 / 7 / 2010$ & 59 & \\
\hline FL & NWFWMD & QUINCY MO\#1 & 304154084453901 & 8152 & Gadsden & 304151 & 844531 & $6 / 7 / 2010$ & 77 & \\
\hline FL & NWFWMD & RAY PETER & 305043086083301 & 5240 & Walton & 305047 & 860853 & $6 / 9 / 2010$ & 185 & \\
\hline FL & NWFWMD & $\begin{array}{l}\text { REDBAY TOWER/AKA } \\
\text { USGS } 43\end{array}$ & 303454085560601 & 3877 & Walton & 303453 & 855604 & $6 / 10 / 2010$ & 23 & \\
\hline FL & NWFWMD & REGISTER/OLD GARLAND & 303845084250001 & 4359 & Gadsden & 303846 & 842460 & $6 / 10 / 2010$ & 58 & \\
\hline FL & NWFWMD & $\begin{array}{l}\text { RU CHOCTAWHATCHEE R. } \\
\text { FLD MONITOR }\end{array}$ & - & 9413 & Walton & 303158 & 855434 & $6 / 10 / 2010$ & 20 & \\
\hline FL & NWFWMD & $\begin{array}{l}\text { RU MONITOR/CEDAR } \\
\text { STREET/S672 }\end{array}$ & - & 8361 & Walton & 303836 & 860633 & $6 / 10 / 2010$ & 66 & \\
\hline FL & NWFWMD & $\begin{array}{l}\text { RU MONITOR/ELEMEN- } \\
\text { TARY SCHOOL }\end{array}$ & - & 8921 & Walton & 303027 & 860744 & $6 / 10 / 2010$ & 10 & \\
\hline FL & NWFWMD & $\begin{array}{l}\text { RU MONITOR/ROCK HILL } \\
\text { APT PROD./DEEP }\end{array}$ & - & 7250 & Walton & 303629 & 860644 & $6 / 10 / 2010$ & 44 & \\
\hline FL & NWFWMD & S.L. MATTHEWS & 302442086130701 & 2034 & Walton & 302441 & 861309 & $6 / 9 / 2010$ & 0 & \\
\hline FL & NWFWMD & $\begin{array}{l}\text { SANDY CAMPBELL / NI- } \\
\text { TRATE POT. MAP }\end{array}$ & 301337084204001 & 728 & Wakulla & 301336 & 842039 & $6 / 8 / 2010$ & 6 & \\
\hline FL & NWFWMD & ST THOMAS SQUARE & - & 563 & Bay & 301005 & 854720 & $6 / 7 / 2010$ & -16 & \\
\hline FL & NWFWMD & STATE HATCHERY \#3 & - & 4788 & Santa Rosa & 304412 & 864758 & $6 / 7 / 2010$ & 13 & \\
\hline FL & NWFWMD & SWU BURNHAM ROAD & - & 7767 & Walton & 302829 & 860226 & $6 / 1 / 2010$ & 15 & \\
\hline FL & NWFWMD & SWU MACK BAYOU & - & 7349 & Walton & 302410 & 861832 & $6 / 9 / 2010$ & -17 & \\
\hline FL & NWFWMD & SWU WEST MONITOR & - & 7183 & Okaloosa & 302344 & 862349 & $6 / 9 / 2010$ & -35 & \\
\hline
\end{tabular}


Table 1. Water-level measurements in Florida and parts of Georgia, South Carolina, and Alabama, May-June 2010.—Continued

[NGVD 29, National Geodetic Vertical Datum of 1929. Abbreviations for agency database: ACEPD, Alachua County Environmental Protection Department; FGS, Florida Geological Survey; GSA, Geological Survey of Alabama; NWFWMD, Northwest Florida Water Management District; SFWMD, South Florida Water Management District; SRWMD, Suwannee River Water Management District; USGSALWSC, U.S. Geological Survey Alabama Water Science Center; USGS-FLWSC, U.S. Geological Survey Florida Water Science Center; GAWSC, U.S. Geological Survey Georgia Water Science Center; SCWSC, U.S. Geological Survey South Carolina Water Science Center. Abbreviations for Station names: BLVD., boulevard; CO, county; CR, county road; E, east; HS, high school; IFAS, Institute of Food and Agricultural Services; LK, lake; MW, monitoring well; N, north; NE, northeast; NE, northeast; NF, national forest; NW, northwest; PK, park; R., river; RD., road; SE, southeast; SE, southeast; SR, state road; ST., street; TWR, tower; UFA, Upper Floridan aquifer; - , not available; \#, number; “, inches]

\begin{tabular}{|c|c|c|c|c|c|c|c|c|c|c|}
\hline State & $\begin{array}{l}\text { Agency } \\
\text { database }\end{array}$ & Station name & $\begin{array}{c}\text { USGS site } \\
\text { identification } \\
\text { number }\end{array}$ & $\begin{array}{l}\text { Local agency } \\
\text { unique site } \\
\text { identifier }\end{array}$ & County & $\begin{array}{l}\text { Latitude } \\
\text { (degree } \\
\text { minutes } \\
\text { and } \\
\text { seconds) } \\
\text { [DDMMSS] }\end{array}$ & $\begin{array}{l}\text { Longitude } \\
\text { (degree } \\
\text { minutes } \\
\text { and } \\
\text { seconds) } \\
\text { [DDMMSS] }\end{array}$ & $\begin{array}{c}\text { Date of } \\
\text { measure- } \\
\text { ment }\end{array}$ & $\begin{array}{c}\text { Water } \\
\text { level } \\
\text { altitude } \\
\text { (feet } \\
\text { above } \\
\text { NGVD 29) }\end{array}$ & Remarks \\
\hline FL & NWFWMD & $\begin{array}{l}\text { SWU-FAF \#47 MONITOR/ } \\
\text { S655 }\end{array}$ & - & 3718 & Walton & 303347 & 860147 & $6 / 10 / 2010$ & 16 & \\
\hline FL & NWFWMD & TAFB \#10/BACKUP\#1 & 300347085345501 & 289 & Bay & 300347 & 853454 & $6 / 10 / 2010$ & 5 & \\
\hline FL & NWFWMD & TOM BROWN TEST & 302613084130001 & 2415 & Leon & 302623 & 841307 & $6 / 8 / 2010$ & 26 & \\
\hline FL & NWFWMD & $\begin{array}{l}\text { UNUSED SOUTH OF CHAT- } \\
\text { TAHOOCHEE }\end{array}$ & 304056084502501 & 4566 & Gadsden & 304056 & 845026 & $6 / 7 / 2010$ & 64 & \\
\hline FL & NWFWMD & USFS PORTER LAKE & 301035084403701 & 589 & Liberty & 301037 & 844037 & $6 / 7 / 2010$ & 32 & \\
\hline FL & NWFWMD & USFS-HICKORY LANDING & 295918085005201 & 226 & Franklin & 295918 & 850049 & $6 / 7 / 2010$ & 18 & \\
\hline FL & NWFWMD & USGS \#39/HOMER HIRT & 304230084535901 & 4681 & Jackson & 304232 & 845401 & $6 / 7 / 2010$ & 76 & \\
\hline FL & NWFWMD & $\begin{array}{l}\text { USGS-422A NEAR GREEN- } \\
\text { HEAD }\end{array}$ & 303025085350501 & 3216 & Washington & 303026 & 853503 & $5 / 20 / 2010$ & 60 & \\
\hline FL & NWFWMD & $\begin{array}{l}\text { USGS-LAKE JACKSON } \\
\text { FLORIDAN }\end{array}$ & 303142084214601 & 3402 & Leon & 303142 & 842145 & $6 / 7 / 2010$ & 36 & \\
\hline FL & NWFWMD & USGS-LESTER LEWIS & 300540084174001 & 342 & Wakulla & 300545 & 841748 & $6 / 8 / 2010$ & 6 & \\
\hline FL & NWFWMD & USGS-OLSON RD./S677 & 303001084134701 & 3156 & Leon & 303002 & 841347 & $6 / 4 / 2010$ & 36 & \\
\hline FL & NWFWMD & VALLEY VIEW SCHOOL & 303803085582201 & 4285 & Walton & 303803 & 855822 & $6 / 10 / 2010$ & 52 & \\
\hline FL & NWFWMD & $\begin{array}{l}\text { VAN BUTLER/USGS WAL- } \\
\text { TON \#21 }\end{array}$ & 301946086095701 & 1074 & Walton & 301948 & 860956 & $6 / 9 / 2010$ & 11 & \\
\hline FL & NWFWMD & W.R. BUCKHALTER & 304918084565601 & 5147 & Jackson & 304932 & 845706 & $6 / 7 / 2010$ & 79 & \\
\hline FL & NWFWMD & $\begin{array}{l}\text { WAKULLA PARKS AND } \\
\text { RECREATION }\end{array}$ & 300655084223701 & 372 & Wakulla & 300656 & 842237 & $6 / 8 / 2010$ & 4 & \\
\hline FL & NWFWMD & WALTER PECEVICH & - & 7208 & Santa Rosa & 303708 & 865400 & $6 / 8 / 2010$ & -3 & \\
\hline FL & NWFWMD & WAUSAU \#1 & 303747085352001 & 5996 & Washington & 303747 & 853520 & $6 / 8 / 2010$ & 37 & \\
\hline FL & NWFWMD & WILLIAM FISHER & - & 4912 & Washington & 304612 & 853038 & $6 / 8 / 2010$ & 65 & \\
\hline FL & NWFWMD & WILLIAMSON/YATES & - & 3867 & Washington & 303450 & 855156 & $6 / 8 / 2010$ & 16 & \\
\hline FL & NWFWMD & WRIGHT ELEMENTARY & 302609086375401 & 2394 & Okaloosa & 302611 & 863757 & $6 / 8 / 2010$ & -80 & \\
\hline
\end{tabular}


Table 1. Water-level measurements in Florida and parts of Georgia, South Carolina, and Alabama, May-June 2010.—Continued

[NGVD 29, National Geodetic Vertical Datum of 1929. Abbreviations for agency database: ACEPD, Alachua County Environmental Protection Department; FGS, Florida Geological Survey; GSA, Geological Survey of Alabama; NWFWMD, Northwest Florida Water Management District; SFWMD, South Florida Water Management District; SRWMD, Suwannee River Water Management District; USGSALWSC, U.S. Geological Survey Alabama Water Science Center; USGS-FLWSC, U.S. Geological Survey Florida Water Science Center; GAWSC, U.S. Geological Survey Georgia Water Science Center; SCWSC, U.S. Geological Survey South Carolina Water Science Center. Abbreviations for Station names: BLVD., boulevard; CO, county; CR, county road; E, east; HS, high school; IFAS, Institute of Food and Agricultural Services; LK, lake; MW, monitoring well; N, north; NE, northeast; NE, northeast; NF, national forest; NW, northwest; PK, park; R., river; RD., road; SE, southeast; SE, southeast; SR, state road; ST., street; TWR, tower; UFA, Upper Floridan aquifer; - - not available; \#, number; “, inches]

\begin{tabular}{|c|c|c|c|c|c|c|c|c|c|c|}
\hline State & $\begin{array}{c}\text { Agency } \\
\text { database }\end{array}$ & Station name & $\begin{array}{c}\text { USGS site } \\
\text { identification } \\
\text { number }\end{array}$ & $\begin{array}{l}\text { Local agency } \\
\text { unique site } \\
\text { identifier }\end{array}$ & County & $\begin{array}{l}\text { Latitude } \\
\text { (degree } \\
\text { minutes } \\
\text { and } \\
\text { seconds) } \\
\text { [DDMMSS] }\end{array}$ & $\begin{array}{l}\text { Longitude } \\
\text { (degree } \\
\text { minutes } \\
\text { and } \\
\text { seconds) } \\
\text { ] [DDMMSS] }\end{array}$ & $\begin{array}{c}\text { Date of } \\
\text { measure- } \\
\text { ment }\end{array}$ & $\begin{array}{c}\text { Water } \\
\text { level } \\
\text { altitude } \\
\text { (feet } \\
\text { above } \\
\text { NGVD 29) }\end{array}$ & Remarks \\
\hline FL & NWFWMD & WRIGHT UPPER FLORIDAN & 302747086382001 & 2822 & Okaloosa & 302748 & 863820 & $6 / 8 / 2010$ & -70 & \\
\hline FL & SFWMD & BF-4S & - & BF-4S & Broward & 261024 & 801047 & $5 / 30 / 2010$ & 43 & $\begin{array}{l}\text { Uncorrected hydrau- } \\
\text { lic head reported; } \\
\text { brackish to saline } \\
\text { water; not con- } \\
\text { toured on Potentio- } \\
\text { metric surface map }\end{array}$ \\
\hline FL & SFWMD & BF-6 & 261901080074601 & BF-6 & Broward & 261852 & 800726 & $5 / 30 / 2010$ & 44 & $\begin{array}{l}\text { Uncorrected hydrau- } \\
\text { lic head reported; } \\
\text { brackish to saline } \\
\text { water; not con- } \\
\text { toured on Potentio- } \\
\text { metric surface map }\end{array}$ \\
\hline FL & SFWMD & BICY-MZ2 & - & BICY-MZ2 & Collier & 255337 & 811833 & $5 / 25 / 2010$ & 39 & $\begin{array}{l}\text { Uncorrected hydrau- } \\
\text { lic head reported; } \\
\text { brackish to saline } \\
\text { water; not con- } \\
\text { toured on Potentio- } \\
\text { metric surface map }\end{array}$ \\
\hline FL & SFWMD & BOYRO_EPXU & - & BOYRO_EPXU & Palm Beach & 262938 & 800530 & $5 / 30 / 2010$ & 46 & $\begin{array}{l}\text { Uncorrected hydrau- } \\
\text { lic head reported; } \\
\text { brackish to saline } \\
\text { water; not con- } \\
\text { toured on Potentio- } \\
\text { metric surface map }\end{array}$ \\
\hline
\end{tabular}


Table 1. Water-level measurements in Florida and parts of Georgia, South Carolina, and Alabama, May-June 2010.—Continued

[NGVD 29, National Geodetic Vertical Datum of 1929. Abbreviations for agency database: ACEPD, Alachua County Environmental Protection Department; FGS, Florida Geological Survey; GSA, Geological Survey of Alabama; NWFWMD, Northwest Florida Water Management District; SFWMD, South Florida Water Management District; SRWMD, Suwannee River Water Management District; USGSALWSC, U.S. Geological Survey Alabama Water Science Center; USGS-FLWSC, U.S. Geological Survey Florida Water Science Center; GAWSC, U.S. Geological Survey Georgia Water Science Center; SCWSC, U.S. Geological Survey South Carolina Water Science Center. Abbreviations for Station names: BLVD., boulevard; CO, county; CR, county road; E, east; HS, high school; IFAS, Institute of Food and Agricultural Services; LK, lake; MW, monitoring well; N, north; NE, northeast; NE, northeast; NF, national forest; NW, northwest; PK, park; R., river; RD., road; SE, southeast; SE, southeast; SR, state road; ST., street; TWR, tower; UFA, Upper Floridan aquifer; - , not available; \#, number; “, inches]

\begin{tabular}{|c|c|c|c|c|c|c|c|c|c|c|}
\hline State & $\begin{array}{c}\text { Agency } \\
\text { database }\end{array}$ & Station name & $\begin{array}{c}\text { USGS site } \\
\text { identification } \\
\text { number }\end{array}$ & $\begin{array}{l}\text { Local agency } \\
\text { unique site } \\
\text { identifier }\end{array}$ & County & $\begin{array}{l}\text { Latitude } \\
\text { (degree } \\
\text { minutes } \\
\text { and } \\
\text { seconds) } \\
\text { [DDMMSS] }\end{array}$ & $\begin{array}{l}\text { Longitude } \\
\text { (degree } \\
\text { minutes } \\
\text { and } \\
\text { seconds) } \\
\text { [DDMMSS] }\end{array}$ & $\begin{array}{c}\text { Date of } \\
\text { measure- } \\
\text { ment }\end{array}$ & $\begin{array}{l}\text { Water } \\
\text { level } \\
\text { altitude } \\
\text { (feet } \\
\text { above } \\
\text { NGVD 29) }\end{array}$ & Remarks \\
\hline FL & SFWMD & BRY-MW & - & BRY-MW & Hendry & 264230 & 812948 & $5 / 30 / 2010$ & 52 & $\begin{array}{l}\text { Uncorrected hydrau- } \\
\text { lic head reported; } \\
\text { brackish to saline } \\
\text { water; not con- } \\
\text { toured on Potentio- } \\
\text { metric surface map }\end{array}$ \\
\hline FL & SFWMD & BSU-MZU & - & BSU-MZU & Charlotte & 264621 & 820209 & $5 / 30 / 2010$ & 42 & $\begin{array}{l}\text { Uncorrected hydrau- } \\
\text { lic head reported; } \\
\text { brackish to saline } \\
\text { water; not con- } \\
\text { toured on Potentio- } \\
\text { metric surface map }\end{array}$ \\
\hline FL & SFWMD & DF-4 & 255434080280901 & DF-4 & Dade & 255436 & 802807 & $5 / 30 / 2010$ & 51 & $\begin{array}{l}\text { Uncorrected hydrau- } \\
\text { lic head reported; } \\
\text { brackish to saline } \\
\text { water; not con- } \\
\text { toured on Potentio- } \\
\text { metric surface map }\end{array}$ \\
\hline FL & SFWMD & ENP-100 & 252255080361101 & ENP-100 & Dade & 252257 & 803611 & $5 / 30 / 2010$ & 38 & $\begin{array}{l}\text { Uncorrected hydrau- } \\
\text { lic head reported; } \\
\text { brackish to saline } \\
\text { water; not con- } \\
\text { toured on Potentio- } \\
\text { metric surface map }\end{array}$ \\
\hline FL & SFWMD & FPL-MW & - & FPL-MW & Lee & 264145 & 814655 & $5 / 30 / 2010$ & 45 & $\begin{array}{l}\text { Uncorrected hydrau- } \\
\text { lic head reported; } \\
\text { brackish to saline } \\
\text { water; not con- } \\
\text { toured on Potentio- } \\
\text { metric surface map }\end{array}$ \\
\hline
\end{tabular}


Table 1. Water-level measurements in Florida and parts of Georgia, South Carolina, and Alabama, May-June 2010.—Continued

[NGVD 29, National Geodetic Vertical Datum of 1929. Abbreviations for agency database: ACEPD, Alachua County Environmental Protection Department; FGS, Florida Geological Survey; GSA, Geological Survey of Alabama; NWFWMD, Northwest Florida Water Management District; SFWMD, South Florida Water Management District; SRWMD, Suwannee River Water Management District; USGSALWSC, U.S. Geological Survey Alabama Water Science Center; USGS-FLWSC, U.S. Geological Survey Florida Water Science Center; GAWSC, U.S. Geological Survey Georgia Water Science Center; SCWSC, U.S. Geological Survey South Carolina Water Science Center. Abbreviations for Station names: BLVD., boulevard; CO, county; CR, county road; E, east; HS, high school; IFAS, Institute of Food and Agricultural Services; LK, lake; MW, monitoring well; N, north; NE, northeast; NE, northeast; NF, national forest; NW, northwest; PK, park; R., river; RD., road; SE, southeast; SE, southeast; SR, state road; ST., street; TWR, tower; UFA, Upper Floridan aquifer; - - not available; \#, number; “, inches]

\begin{tabular}{|c|c|c|c|c|c|c|c|c|c|c|}
\hline State & $\begin{array}{c}\text { Agency } \\
\text { database }\end{array}$ & Station name & $\begin{array}{c}\text { USGS site } \\
\text { identification } \\
\text { number }\end{array}$ & $\begin{array}{l}\text { Local agency } \\
\text { unique site } \\
\text { identifier }\end{array}$ & County & $\begin{array}{l}\text { Latitude } \\
\text { (degree } \\
\text { minutes } \\
\text { and } \\
\text { seconds) } \\
\text { [DDMMSS] }\end{array}$ & $\begin{array}{l}\text { Longitude } \\
\text { (degree } \\
\text { minutes } \\
\text { and } \\
\text { seconds) } \\
\text { [DDMMSS] }\end{array}$ & $\begin{array}{c}\text { Date of } \\
\text { measure- } \\
\text { ment }\end{array}$ & $\begin{array}{c}\text { Water } \\
\text { level } \\
\text { altitude } \\
\text { (feet } \\
\text { above } \\
\text { NGVD 29) }\end{array}$ & Remarks \\
\hline FL & SFWMD & FPU-MZU & 272720080182702 & FPU-MZU & St Lucie & 272721 & 801855 & $5 / 30 / 2010$ & 36 & $\begin{array}{l}\text { Uncorrected hydrau- } \\
\text { lic head reported; } \\
\text { brackish to saline } \\
\text { water; not con- } \\
\text { toured on Potentio- } \\
\text { metric surface map }\end{array}$ \\
\hline FL & SFWMD & G-2618 & 261016080492601 & G-2618 & Broward & 261017 & 804919 & $5 / 30 / 2010$ & 58 & $\begin{array}{l}\text { Uncorrected hydrau- } \\
\text { lic head reported; } \\
\text { brackish to saline } \\
\text { water; not con- } \\
\text { toured on Potentio- } \\
\text { metric surface map }\end{array}$ \\
\hline FL & SFWMD & G-3061 & 254941080171701 & G-3061 & Dade & 254943 & 801716 & $5 / 30 / 2010$ & 44 & $\begin{array}{l}\text { Uncorrected hydrau- } \\
\text { lic head reported; } \\
\text { brackish to saline } \\
\text { water; not con- } \\
\text { toured on Potentio- } \\
\text { metric surface map }\end{array}$ \\
\hline FL & SFWMD & HIF-40 & 271618081120801 & HIF-40 & Highlands & 271618 & 811208 & $5 / 30 / 2010$ & 46 & $\begin{array}{l}\text { Uncorrected hydrau- } \\
\text { lic head reported; } \\
\text { brackish to saline } \\
\text { water; not con- } \\
\text { toured on Potentio- } \\
\text { metric surface map }\end{array}$ \\
\hline FL & SFWMD & HIF-42U & - & HIF-42U & Okeechobee & 271311 & 805722 & $5 / 30 / 2010$ & 47 & $\begin{array}{l}\text { Uncorrected hydrau- } \\
\text { lic head reported; } \\
\text { brackish to saline } \\
\text { water; not con- } \\
\text { toured on Potentio- } \\
\text { metric surface map }\end{array}$ \\
\hline
\end{tabular}


Table 1. Water-level measurements in Florida and parts of Georgia, South Carolina, and Alabama, May-June 2010.—Continued

[NGVD 29, National Geodetic Vertical Datum of 1929. Abbreviations for agency database: ACEPD, Alachua County Environmental Protection Department; FGS, Florida Geological Survey; GSA, Geological Survey of Alabama; NWFWMD, Northwest Florida Water Management District; SFWMD, South Florida Water Management District; SRWMD, Suwannee River Water Management District; USGSALWSC, U.S. Geological Survey Alabama Water Science Center; USGS-FLWSC, U.S. Geological Survey Florida Water Science Center; GAWSC, U.S. Geological Survey Georgia Water Science Center; SCWSC, U.S. Geological Survey South Carolina Water Science Center. Abbreviations for Station names: BLVD., boulevard; CO, county; CR, county road; E, east; HS, high school; IFAS, Institute of Food and Agricultural Services; LK, lake; MW, monitoring well; N, north; NE, northeast; NE, northeast; NF, national forest; NW, northwest; PK, park; R., river; RD., road; SE, southeast; SE, southeast; SR, state road; ST., street; TWR, tower; UFA, Upper Floridan aquifer; - , not available; \#, number; “, inches]

\begin{tabular}{|c|c|c|c|c|c|c|c|c|c|c|}
\hline State & $\begin{array}{c}\text { Agency } \\
\text { database }\end{array}$ & Station name & $\begin{array}{c}\text { USGS site } \\
\text { identification } \\
\text { number }\end{array}$ & $\begin{array}{l}\text { Local agency } \\
\text { unique site } \\
\text { identifier }\end{array}$ & County & $\begin{array}{c}\text { Latitude } \\
\text { (degree } \\
\text { minutes } \\
\text { and } \\
\text { seconds) } \\
\text { [DDMMSS] }\end{array}$ & $\begin{array}{l}\text { Longitude } \\
\text { (degree } \\
\text { minutes } \\
\text { and } \\
\text { seconds) } \\
\text { ] [DDMMSS] }\end{array}$ & $\begin{array}{c}\text { Date of } \\
\text { measure- } \\
\text { ment }\end{array}$ & $\begin{array}{c}\text { Water } \\
\text { level } \\
\text { altitude } \\
\text { (feet } \\
\text { above } \\
\text { NGVD 29) }\end{array}$ & Remarks \\
\hline FL & SFWMD & I75-MZ2 (upper floridan) & 261012081435101 & $\begin{array}{l}\text { I75-MZ2 (upper } \\
\text { floridan) }\end{array}$ & Collier & 261013 & 814350 & $5 / 30 / 2010$ & 32 & $\begin{array}{l}\text { Uncorrected hydrau- } \\
\text { lic head reported; } \\
\text { brackish to saline } \\
\text { water; not con- } \\
\text { toured on Potentio- } \\
\text { metric surface map }\end{array}$ \\
\hline FL & SFWMD & IWA-MZU & - & IWA-MZU & Lee & 262632 & 820638 & $5 / 30 / 2010$ & 18 & $\begin{array}{l}\text { Uncorrected hydrau- } \\
\text { lic head reported; } \\
\text { brackish to saline } \\
\text { water; not con- } \\
\text { toured on Potentio- } \\
\text { metric surface map }\end{array}$ \\
\hline FL & SFWMD & IWSD-MZ2 & 262448081255401 & IWSD-MZ2 & Collier & 262450 & 812553 & $5 / 30 / 2010$ & 55 & $\begin{array}{l}\text { Uncorrected hydrau- } \\
\text { lic head reported; } \\
\text { brackish to saline } \\
\text { water; not con- } \\
\text { toured on Potentio- } \\
\text { metric surface map }\end{array}$ \\
\hline FL & SFWMD & KW-MZL & - & KW-MZL & Monroe & 243407 & 814747 & $5 / 30 / 2010$ & 8 & $\begin{array}{l}\text { Uncorrected hydrau- } \\
\text { lic head reported; } \\
\text { brackish to saline } \\
\text { water; not con- } \\
\text { toured on Potentio- } \\
\text { metric surface map }\end{array}$ \\
\hline FL & SFWMD & L2-PW2 & 263627080565802 & L2-PW2 & Hendry & 263629 & 805658 & $5 / 30 / 2010$ & 58 & $\begin{array}{l}\text { Uncorrected hydrau- } \\
\text { lic head reported; } \\
\text { brackish to saline } \\
\text { water; not con- } \\
\text { toured on Potentio- } \\
\text { metric surface map }\end{array}$ \\
\hline
\end{tabular}


Table 1. Water-level measurements in Florida and parts of Georgia, South Carolina, and Alabama, May-June 2010.—Continued

[NGVD 29, National Geodetic Vertical Datum of 1929. Abbreviations for agency database: ACEPD, Alachua County Environmental Protection Department; FGS, Florida Geological Survey; GSA, Geological Survey of Alabama; NWFWMD, Northwest Florida Water Management District; SFWMD, South Florida Water Management District; SRWMD, Suwannee River Water Management District; USGSALWSC, U.S. Geological Survey Alabama Water Science Center; USGS-FLWSC, U.S. Geological Survey Florida Water Science Center; GAWSC, U.S. Geological Survey Georgia Water Science Center; SCWSC, U.S. Geological Survey South Carolina Water Science Center. Abbreviations for Station names: BLVD., boulevard; CO, county; CR, county road; E, east; HS, high school; IFAS, Institute of Food and Agricultural Services; LK, lake; MW, monitoring well; N, north; NE, northeast; NE, northeast; NF, national forest; NW, northwest; PK, park; R., river; RD., road; SE, southeast; SE, southeast; SR, state road; ST., street; TWR, tower; UFA, Upper Floridan aquifer; - , not available; \#, number; “, inches]

\begin{tabular}{|c|c|c|c|c|c|c|c|c|c|c|}
\hline State & $\begin{array}{c}\text { Agency } \\
\text { database }\end{array}$ & Station name & $\begin{array}{c}\text { USGS site } \\
\text { identification } \\
\text { number }\end{array}$ & $\begin{array}{l}\text { Local agency } \\
\text { unique site } \\
\text { identifier }\end{array}$ & County & $\begin{array}{c}\text { Latitude } \\
\text { (degree } \\
\text { minutes } \\
\text { and } \\
\text { seconds) } \\
\text { [DDMMSS] }\end{array}$ & $\begin{array}{l}\text { Longitude } \\
\text { (degree } \\
\text { minutes } \\
\text { and } \\
\text { seconds) } \\
\text { [DDMMSS] }\end{array}$ & $\begin{array}{c}\text { Date of } \\
\text { measure- } \\
\text { ment }\end{array}$ & $\begin{array}{c}\text { Water } \\
\text { level } \\
\text { altitude } \\
\text { (feet } \\
\text { above } \\
\text { NGVD 29) }\end{array}$ & Remarks \\
\hline FL & SFWMD & L-6436 & 263906082032101 & L-6436 & Lee & 263907 & 820320 & $6 / 1 / 2010$ & 32 & $\begin{array}{l}\text { Uncorrected hydrau- } \\
\text { lic head reported; } \\
\text { brackish to saline } \\
\text { water; not con- } \\
\text { toured on Potentio- } \\
\text { metric surface map }\end{array}$ \\
\hline FL & SFWMD & LAB-MZ1 & - & LAB-MZ1 & Hendry & 264511 & 812818 & $5 / 30 / 2010$ & 53 & $\begin{array}{l}\text { Uncorrected hydrau- } \\
\text { lic head reported; } \\
\text { brackish to saline } \\
\text { water; not con- } \\
\text { toured on Potentio- } \\
\text { metric surface map }\end{array}$ \\
\hline FL & SFWMD & $M F-35 B$ & - & MF-35B & Martin & 265929 & 802858 & $5 / 30 / 2010$ & 52 & $\begin{array}{l}\text { Uncorrected hydrau- } \\
\text { lic head reported; } \\
\text { brackish to saline } \\
\text { water; not con- } \\
\text { toured on Potentio- } \\
\text { metric surface map }\end{array}$ \\
\hline FL & SFWMD & MF-37U & 265928080362801 & MF-37U & Martin & 265926 & 803617 & $5 / 30 / 2010$ & 52 & $\begin{array}{l}\text { Uncorrected hydrau- } \\
\text { lic head reported; } \\
\text { brackish to saline } \\
\text { water; not con- } \\
\text { toured on Potentio- } \\
\text { metric surface map }\end{array}$ \\
\hline FL & SFWMD & MF-40U & - & MF-40U & Martin & 271221 & 802832 & $5 / 30 / 2010$ & 49 & $\begin{array}{l}\text { Uncorrected hydrau- } \\
\text { lic head reported; } \\
\text { brackish to saline } \\
\text { water; not con- } \\
\text { toured on Potentio- } \\
\text { metric surface map }\end{array}$ \\
\hline
\end{tabular}


Table 1. Water-level measurements in Florida and parts of Georgia, South Carolina, and Alabama, May-June 2010.—Continued

[NGVD 29, National Geodetic Vertical Datum of 1929. Abbreviations for agency database: ACEPD, Alachua County Environmental Protection Department; FGS, Florida Geological Survey; GSA, Geological Survey of Alabama; NWFWMD, Northwest Florida Water Management District; SFWMD, South Florida Water Management District; SRWMD, Suwannee River Water Management District; USGSALWSC, U.S. Geological Survey Alabama Water Science Center; USGS-FLWSC, U.S. Geological Survey Florida Water Science Center; GAWSC, U.S. Geological Survey Georgia Water Science Center; SCWSC, U.S. Geological Survey South Carolina Water Science Center. Abbreviations for Station names: BLVD., boulevard; CO, county; CR, county road; E, east; HS, high school; IFAS, Institute of Food and Agricultural Services; LK, lake; MW, monitoring well; N, north; NE, northeast; NE, northeast; NF, national forest; NW, northwest; PK, park; R., river; RD., road; SE, southeast; SE, southeast; SR, state road; ST., street; TWR, tower; UFA, Upper Floridan aquifer; - , not available; \#, number; “, inches]

\begin{tabular}{|c|c|c|c|c|c|c|c|c|c|c|}
\hline State & $\begin{array}{c}\text { Agency } \\
\text { database }\end{array}$ & Station name & $\begin{array}{c}\text { USGS site } \\
\text { identification } \\
\text { number }\end{array}$ & $\begin{array}{l}\text { Local agency } \\
\text { unique site } \\
\text { identifier }\end{array}$ & County & $\begin{array}{c}\text { Latitude } \\
\text { (degree } \\
\text { minutes } \\
\text { and } \\
\text { seconds) } \\
\text { [DDMMSS] }\end{array}$ & $\begin{array}{l}\text { Longitude } \\
\text { (degree } \\
\text { minutes } \\
\text { and } \\
\text { seconds) } \\
\text { [DDMMSS] }\end{array}$ & $\begin{array}{c}\text { Date of } \\
\text { measure- } \\
\text { ment }\end{array}$ & $\begin{array}{c}\text { Water } \\
\text { level } \\
\text { altitude } \\
\text { (feet } \\
\text { above } \\
\text { NGVD 29) }\end{array}$ & Remarks \\
\hline $\mathrm{FL}$ & SFWMD & MF-52 & 270512080230801 & MF-52 & Martin & 270506 & 802308 & $5 / 30 / 2010$ & 49 & $\begin{array}{l}\text { Uncorrected hydrau- } \\
\text { lic head reported; } \\
\text { brackish to saline } \\
\text { water; not con- } \\
\text { toured on Potentio- } \\
\text { metric surface map }\end{array}$ \\
\hline FL & SFWMD & MOSSPK_D & 282241081112801 & MOSSPK_D & Orange & 282242 & 811127 & $5 / 30 / 2010$ & 41 & \\
\hline FL & SFWMD & OKF-100U & - & OKF-100U & Glades & 270917 & 805216 & $5 / 30 / 2010$ & 58 & $\begin{array}{l}\text { Uncorrected hydrau- } \\
\text { lic head reported; } \\
\text { brackish to saline } \\
\text { water; not con- } \\
\text { toured on Potentio- } \\
\text { metric surface map }\end{array}$ \\
\hline FL & SFWMD & OKF-101 & - & OKF-101 & Okeechobee & 271152 & 805023 & $5 / 30 / 2010$ & 47 & $\begin{array}{l}\text { Uncorrected hydrau- } \\
\text { lic head reported; } \\
\text { brackish to saline } \\
\text { water; not con- } \\
\text { toured on Potentio- } \\
\text { metric surface map }\end{array}$ \\
\hline FL & SFWMD & OKF-105U & - & OKF-105U & Okeechobee & 272407 & 810651 & $5 / 30 / 2010$ & 46 & $\begin{array}{l}\text { Uncorrected hydrau- } \\
\text { lic head reported; } \\
\text { brackish to saline } \\
\text { water; not con- } \\
\text { toured on Potentio- } \\
\text { metric surface map }\end{array}$ \\
\hline
\end{tabular}


Table 1. Water-level measurements in Florida and parts of Georgia, South Carolina, and Alabama, May-June 2010.-Continued

[NGVD 29, National Geodetic Vertical Datum of 1929. Abbreviations for agency database: ACEPD, Alachua County Environmental Protection Department; FGS, Florida Geological Survey; GSA, Geological Survey of Alabama; NWFWMD, Northwest Florida Water Management District; SFWMD, South Florida Water Management District; SRWMD, Suwannee River Water Management District; USGSALWSC, U.S. Geological Survey Alabama Water Science Center; USGS-FLWSC, U.S. Geological Survey Florida Water Science Center; GAWSC, U.S. Geological Survey Georgia Water Science Center; SCWSC, U.S. Geological Survey South Carolina Water Science Center. Abbreviations for Station names: BLVD., boulevard; CO, county; CR, county road; E, east; HS, high school; IFAS, Institute of Food and Agricultural Services; LK, lake; MW, monitoring well; N, north; NE, northeast; NE, northeast; NF, national forest; NW, northwest; PK, park; R., river; RD., road; SE, southeast; SE, southeast; SR, state road; ST., street; TWR, tower; UFA, Upper Floridan aquifer; - , not available; \#, number; “, inches]

\begin{tabular}{|c|c|c|c|c|c|c|c|c|c|c|}
\hline State & $\begin{array}{c}\text { Agency } \\
\text { database }\end{array}$ & Station name & $\begin{array}{c}\text { USGS site } \\
\text { identification } \\
\text { number }\end{array}$ & $\begin{array}{c}\text { Local agency } \\
\text { unique site } \\
\text { identifier }\end{array}$ & County & $\begin{array}{c}\text { Latitude } \\
\text { (degree } \\
\text { minutes } \\
\text { and } \\
\text { seconds) } \\
\text { [DDMMSS] }\end{array}$ & $\begin{array}{l}\text { Longitude } \\
\text { (degree } \\
\text { minutes } \\
\text { and } \\
\text { seconds) } \\
\text { [DDMMSS] }\end{array}$ & $\begin{array}{c}\text { Date of } \\
\text { measure- } \\
\text { ment }\end{array}$ & $\begin{array}{c}\text { Water } \\
\text { level } \\
\text { altitude } \\
\text { (feet } \\
\text { above } \\
\text { NGVD 29) }\end{array}$ & Remarks \\
\hline
\end{tabular}

\begin{tabular}{lllllllll}
\hline FL & SFWMD & OKF-106 & - & OKF-106 & Okeechobee & $271416 \quad 804708$ & $5 / 30 / 2010$ & 45
\end{tabular}
lic head reported; brackish to saline water; not contoured on Potentio-

$\begin{array}{lll}\text { FL } & \text { SFWMD } & \text { ORF-61 } \\ \text { FL } & \text { SFWMD } & \text { ORF-62 } \\ \text { FL } & \text { SFWMD } & \text { OSF-101 } \\ \text { FL } & \text { SFWMD } & \text { OSF-102 } \\ \text { FL } & \text { SFWMD } & \text { OSF-103 } \\ \text { FL } & \text { SFWMD } & \text { OSF-60 } \\ \text { FL } & \text { SFWMD } & \text { OSF-62 } \\ \text { FL } & \text { SFWMD } & \text { OSF-64 } \\ \text { FL } & \text { SFWMD } & \text { OSF-66 } \\ \text { FL } & \text { SFWMD } & \text { OSF-70R } \\ \text { FL } & \text { SFWMD } & \text { PB-747 }\end{array}$

282818081320503
282339081093901
281726081265401
280918081252801
282002081380101
274149080534801
275347081022601
280418081160401
280141081112701
281506081194601
265604080082601

$\begin{array}{cllllr}\text { ORF-61 } & \text { Orange } & 282818 & 813205 & 5 / 30 / 2010 & 77 \\ \text { ORF-62 } & \text { Orange } & 282341 & 810941 & 5 / 30 / 2010 & 31 \\ \text { OSF-101 } & \text { Osceola } & 281726 & 812654 & 5 / 30 / 2010 & 56 \\ \text { OSF-102 } & \text { Osceola } & 280918 & 812528 & 5 / 30 / 2010 & 56 \\ \text { OSF-103 } & \text { Osceola } & 282003 & 813801 & 5 / 30 / 2010 & 102 \\ \text { OSF-60 } & \text { Osceola } & 274148 & 805346 & 5 / 30 / 2010 & 43 \\ \text { OSF-62 } & \text { Osceola } & 275403 & 810242 & 5 / 30 / 2010 & 43 \\ \text { OSF-64 } & \text { Osceola } & 280421 & 811647 & 5 / 30 / 2010 & 51 \\ \text { OSF-66 } & \text { Osceola } & 280138 & 811123 & 5 / 30 / 2010 & 48 \\ \text { OSF-70R } & \text { Osceola } & 281509 & 811942 & 5 / 30 / 2010 & 45 \\ \text { PB-747 } & \text { Palm Beach } & 265606 & 800824 & 5 / 30 / 2010 & 48\end{array}$

Uncorrected hydraulic head reported; brackish to saline water; not contoured on Potentiometric surface map

Uncorrected hydraulic head reported; brackish to saline water; not contoured on Potentiometric surface map 
Table 1. Water-level measurements in Florida and parts of Georgia, South Carolina, and Alabama, May-June 2010.—Continued

[NGVD 29, National Geodetic Vertical Datum of 1929. Abbreviations for agency database: ACEPD, Alachua County Environmental Protection Department; FGS, Florida Geological Survey; GSA, Geological Survey of Alabama; NWFWMD, Northwest Florida Water Management District; SFWMD, South Florida Water Management District; SRWMD, Suwannee River Water Management District; USGSALWSC, U.S. Geological Survey Alabama Water Science Center; USGS-FLWSC, U.S. Geological Survey Florida Water Science Center; GAWSC, U.S. Geological Survey Georgia Water Science Center; SCWSC, U.S. Geological Survey South Carolina Water Science Center. Abbreviations for Station names: BLVD., boulevard; CO, county; CR, county road; E, east; HS, high school; IFAS, Institute of Food and Agricultural Services; LK, lake; MW, monitoring well; N, north; NE, northeast; NE, northeast; NF, national forest; NW, northwest; PK, park; R., river; RD., road; SE, southeast; SE, southeast; SR, state road; ST., street; TWR, tower; UFA, Upper Floridan aquifer; —, not available; \#, number; “, inches]

\begin{tabular}{|c|c|c|c|c|c|c|c|c|c|c|}
\hline State & $\begin{array}{c}\text { Agency } \\
\text { database }\end{array}$ & Station name & $\begin{array}{c}\text { USGS site } \\
\text { identification } \\
\text { number }\end{array}$ & $\begin{array}{l}\text { Local agency } \\
\text { unique site } \\
\text { identifier }\end{array}$ & County & $\begin{array}{l}\text { Latitude } \\
\text { (degree } \\
\text { minutes } \\
\text { and } \\
\text { seconds) } \\
\text { [DDMMSS] }\end{array}$ & $\begin{array}{l}\text { Longitude } \\
\text { (degree } \\
\text { minutes } \\
\text { and } \\
\text { seconds) } \\
\text { ] [DDMMSS] }\end{array}$ & $\begin{array}{l}\text { Date of } \\
\text { measure- } \\
\text { ment }\end{array}$ & $\begin{array}{c}\text { Water } \\
\text { level } \\
\text { altitude } \\
\text { (feet } \\
\text { above } \\
\text { NGVD 29) }\end{array}$ & Remarks \\
\hline FL & SFWMD & PBF-2 & 264259080032001 & PBF-2 & Palm Beach & 264217 & 800348 & $5 / 30 / 2010$ & 45 & $\begin{array}{l}\text { Uncorrected hydrau- } \\
\text { lic head reported; } \\
\text { brackish to saline } \\
\text { water; not con- } \\
\text { toured on Potentio- } \\
\text { metric surface map }\end{array}$ \\
\hline FL & SFWMD & PBF-3 & 264033080061101 & PBF-3 & Palm Beach & 264034 & 800609 & $6 / 8 / 2010$ & 46 & $\begin{array}{l}\text { Uncorrected hydrau- } \\
\text { lic head reported; } \\
\text { brackish to saline } \\
\text { water; not con- } \\
\text { toured on Potentio- } \\
\text { metric surface map }\end{array}$ \\
\hline FL & SFWMD & PBF-7U & 264158080425701 & PBF-7U & Palm Beach & 264159 & 804257 & $5 / 30 / 2010$ & 55 & $\begin{array}{l}\text { Uncorrected hydrau- } \\
\text { lic head reported; } \\
\text { brackish to saline } \\
\text { water; not con- } \\
\text { toured on Potentio- } \\
\text { metric surface map }\end{array}$ \\
\hline FL & SFWMD & POF-20R & 273934081075801 & POF-20R & Polk & 273934 & 810759 & $5 / 30 / 2010$ & 45 & \\
\hline FL & SFWMD & POF-22 & 280253081235804 & POF-22 & Polk & 280254 & 812358 & $5 / 30 / 2010$ & 60 & \\
\hline FL & SFWMD & POF-23 & 274454081110201 & POF-23 & Polk & 274454 & 811102 & $5 / 30 / 2010$ & 45 & \\
\hline FL & SFWMD & SCC-MZU & - & SCC-MZU & Collier & 260544 & 814324 & $5 / 30 / 2010$ & 35 & $\begin{array}{l}\text { Uncorrected hydrau- } \\
\text { lic head reported; } \\
\text { brackish to saline } \\
\text { water; not con- } \\
\text { toured on Potentio- } \\
\text { metric surface map }\end{array}$ \\
\hline FL & SFWMD & SKYLAKE_G & 282640081230701 & SKYLAKE_G & Orange & 282640 & 812307 & $5 / 30 / 2010$ & 46 & \\
\hline
\end{tabular}


Table 1. Water-level measurements in Florida and parts of Georgia, South Carolina, and Alabama, May-June 2010.-Continued

[NGVD 29, National Geodetic Vertical Datum of 1929. Abbreviations for agency database: ACEPD, Alachua County Environmental Protection Department; FGS, Florida Geological Survey; GSA, Geological Survey of Alabama; NWFWMD, Northwest Florida Water Management District; SFWMD, South Florida Water Management District; SRWMD, Suwannee River Water Management District; USGSALWSC, U.S. Geological Survey Alabama Water Science Center; USGS-FLWSC, U.S. Geological Survey Florida Water Science Center; GAWSC, U.S. Geological Survey Georgia Water Science Center; SCWSC, U.S. Geological Survey South Carolina Water Science Center. Abbreviations for Station names: BLVD., boulevard; CO, county; CR, county road; E, east; HS, high school; IFAS, Institute of Food and Agricultural Services; LK, lake; MW, monitoring well; N, north; NE, northeast; NE, northeast; NF, national forest; NW, northwest; PK, park; R., river; RD., road; SE, southeast; SE, southeast; SR, state road; ST., street; TWR, tower; UFA, Upper Floridan aquifer; - , not available; \#, number; “, inches]

\begin{tabular}{|c|c|c|c|c|c|c|c|c|c|c|}
\hline State & $\begin{array}{c}\text { Agency } \\
\text { database }\end{array}$ & Station name & $\begin{array}{c}\text { USGS site } \\
\text { identification } \\
\text { number }\end{array}$ & $\begin{array}{l}\text { Local agency } \\
\text { unique site } \\
\text { identifier }\end{array}$ & County & $\begin{array}{l}\text { Latitude } \\
\text { (degree } \\
\text { minutes } \\
\text { and } \\
\text { seconds) } \\
\text { [DDMMSS] }\end{array}$ & $\begin{array}{l}\text { Longitude } \\
\text { (degree } \\
\text { minutes } \\
\text { and } \\
\text { seconds) } \\
\text { ] [DDMMSS] }\end{array}$ & $\begin{array}{c}\text { Date of } \\
\text { measure- } \\
\text { ment }\end{array}$ & $\begin{array}{c}\text { Water } \\
\text { level } \\
\text { altitude } \\
\text { (feet } \\
\text { above } \\
\text { NGVD 29) }\end{array}$ & Remarks \\
\hline FL & SFWMD & SLF-21 & 272537080240901 & SLF-21 & St Lucie & 272542 & 802407 & $5 / 30 / 2010$ & 35 & $\begin{array}{l}\text { Uncorrected hydrau- } \\
\text { lic head reported; } \\
\text { brackish to saline } \\
\text { water; not con- } \\
\text { toured on Potentio- } \\
\text { metric surface map }\end{array}$ \\
\hline FL & SFWMD & SLF-75 & 272015080292402 & SLF-75 & St Lucie & 272016 & 802923 & $5 / 30 / 2010$ & 41 & $\begin{array}{l}\text { Uncorrected hydrau- } \\
\text { lic head reported; } \\
\text { brackish to saline } \\
\text { water; not con- } \\
\text { toured on Potentio- } \\
\text { metric surface map }\end{array}$ \\
\hline FL & SFWMD & SLF-76 & 272015080292403 & SLF-76 & St Lucie & 272016 & 802623 & $5 / 30 / 2010$ & 41 & $\begin{array}{l}\text { Uncorrected hydrau- } \\
\text { lic head reported; } \\
\text { brackish to saline } \\
\text { water; not con- } \\
\text { toured on Potentio- } \\
\text { metric surface map }\end{array}$ \\
\hline FL & SRWMD & +010616001 & 302928083431901 & +010616001 & Madison & 302928 & 834319 & $5 / 10 / 2010$ & 76 & \\
\hline FL & SRWMD & +010719001 & 302824083400901 & +010719001 & Madison & 302827 & 834017 & $5 / 10 / 2010$ & 77 & \\
\hline FL & SRWMD & +010722002 & - & +010722002 & Madison & 302835 & 833614 & $5 / 10 / 2010$ & 75 & \\
\hline FL & SRWMD & +011022001 & 302806083180901 & +011022001 & Madison & 302807 & 831809 & $5 / 11 / 2010$ & 54 & \\
\hline FL & SRWMD & +011028003 & - & +011028003 & Madison & 302712 & 831956 & $5 / 11 / 2010$ & 58 & \\
\hline FL & SRWMD & +011109001 & - & +011109001 & Hamilton & 302945 & 831350 & $5 / 13 / 2010$ & 50 & \\
\hline FL & SRWMD & +011202001 & - & +011202001 & Hamilton & 303121 & 830500 & $5 / 13 / 2010$ & 41 & \\
\hline FL & SRWMD & +011234001 & 302642083065201 & +011234001 & Hamilton & 302642 & 830652 & $5 / 13 / 2010$ & 34 & \\
\hline FL & SRWMD & +011316001 & - & +011316001 & Hamilton & 302900 & 830149 & $5 / 20 / 2010$ & 44 & \\
\hline FL & SRWMD & +011421001 & 302835082545301 & +011421001 & Hamilton & 302835 & 825453 & $5 / 13 / 2010$ & 46 & \\
\hline
\end{tabular}


Table 1. Water-level measurements in Florida and parts of Georgia, South Carolina, and Alabama, May-June 2010.—Continued

[NGVD 29, National Geodetic Vertical Datum of 1929. Abbreviations for agency database: ACEPD, Alachua County Environmental Protection Department; FGS, Florida Geological Survey; GSA, Geological Survey of Alabama; NWFWMD, Northwest Florida Water Management District; SFWMD, South Florida Water Management District; SRWMD, Suwannee River Water Management District; USGSALWSC, U.S. Geological Survey Alabama Water Science Center; USGS-FLWSC, U.S. Geological Survey Florida Water Science Center; GAWSC, U.S. Geological Survey Georgia Water Science Center; SCWSC, U.S. Geological Survey South Carolina Water Science Center. Abbreviations for Station names: BLVD., boulevard; CO, county; CR, county road; E, east; HS, high school; IFAS, Institute of Food and Agricultural Services; LK, lake; MW, monitoring well; N, north; NE, northeast; NE, northeast; NF, national forest; NW, northwest; PK, park; R., river; RD., road; SE, southeast; SE, southeast; SR, state road; ST., street; TWR, tower; UFA, Upper Floridan aquifer; —, not available; \#, number; “, inches]

\begin{tabular}{|c|c|c|c|c|c|c|c|c|c|c|}
\hline State & $\begin{array}{c}\text { Agency } \\
\text { database }\end{array}$ & Station name & $\begin{array}{c}\text { USGS site } \\
\text { identification } \\
\text { number }\end{array}$ & $\begin{array}{l}\text { Local agency } \\
\text { unique site } \\
\text { identifier }\end{array}$ & County & $\begin{array}{l}\text { Latitude } \\
\text { (degree } \\
\text { minutes } \\
\text { and } \\
\text { seconds) } \\
\text { [DDMMSS] }\end{array}$ & $\begin{array}{l}\text { Longitude } \\
\text { (degree } \\
\text { minutes } \\
\text { and } \\
\text { seconds) } \\
\text { [DDMMSS] }\end{array}$ & $\begin{array}{c}\text { Date of } \\
\text { measure- } \\
\text { ment }\end{array}$ & $\begin{array}{c}\text { Water } \\
\text { level } \\
\text { altitude } \\
\text { (feet } \\
\text { above } \\
\text { NGVD 29) }\end{array}$ & Remarks \\
\hline FL & SRWMD & +011422007 & - & +011422007 & Hamilton & 302833 & 825429 & $5 / 12 / 2010$ & 46 & \\
\hline FL & SRWMD & +011510003 & - & +011510003 & Hamilton & 302960 & 824808 & $5 / 12 / 2010$ & 51 & \\
\hline FL & SRWMD & +011608001 & 302957082441201 & +011608001 & Hamilton & 302958 & 824412 & $5 / 12 / 2010$ & 60 & \\
\hline FL & SRWMD & +011714002 & - & +011714002 & Columbia & 302932 & 823528 & $5 / 5 / 2010$ & 52 & \\
\hline FL & SRWMD & +020611001 & - & +020611001 & Jefferson & 303544 & 834155 & $5 / 19 / 2010$ & 71 & \\
\hline FL & SRWMD & +020611002 & - & +020611002 & Jefferson & 303545 & 834157 & $5 / 19 / 2010$ & 75 & \\
\hline FL & SRWMD & +020820001 & 303332083330201 & +020820001 & Madison & 303333 & 833302 & $5 / 11 / 2010$ & 86 & \\
\hline FL & SRWMD & +020822002 & - & +020822002 & Madison & 303400 & 833053 & $5 / 10 / 2010$ & 78 & \\
\hline FL & SRWMD & +021002001 & 303626083172001 & +021002001 & Madison & 303625 & 831715 & $5 / 11 / 2010$ & 63 & \\
\hline FL & SRWMD & +021035003 & - & +021035003 & Madison & 303212 & 831741 & $5 / 12 / 2010$ & 55 & \\
\hline FL & SRWMD & +021036001 & - & +021036001 & Madison & 303132 & 831560 & $5 / 11 / 2010$ & 53 & \\
\hline FL & SRWMD & +021125001 & - & +021125001 & Hamilton & 303224 & 831017 & $5 / 12 / 2010$ & 48 & \\
\hline FL & SRWMD & +021211001 & - & +021211001 & Hamilton & 303511 & 830546 & $5 / 12 / 2010$ & 45 & \\
\hline FL & SRWMD & +021332004 & - & +021332004 & Hamilton & 303152 & 830230 & $5 / 12 / 2010$ & 46 & \\
\hline FL & SRWMD & +021432001 & 303158082562901 & +021432001 & Hamilton & 303201 & 825631 & $5 / 12 / 2010$ & 46 & \\
\hline FL & SRWMD & +021713001 & - & +021713001 & Columbia & 303436 & 823408 & $5 / 5 / 2010$ & 52 & \\
\hline FL & SRWMD & +030524001 & - & +030524001 & Jefferson & 303843 & 834647 & $5 / 19 / 2010$ & 67 & \\
\hline FL & SRWMD & +030727001 & 303812083362401 & +030727001 & Jefferson & 303813 & 833624 & $5 / 19 / 2010$ & 77 & \\
\hline FL & SRWMD & +031133001 & - & +031133001 & Hamilton & 303651 & 831327 & $5 / 13 / 2010$ & 69 & \\
\hline FL & SRWMD & -010833001 & 302100082314701 & -010833001 & Madison & 302101 & 833147 & $5 / 10 / 2010$ & 86 & \\
\hline FL & SRWMD & -010911003 & - & -010911003 & Madison & 302437 & 832336 & $5 / 10 / 2010$ & 79 & \\
\hline FL & SRWMD & -010920002 & 302259083263701 & -010920002 & Madison & 302306 & 832638 & $5 / 10 / 2010$ & 88 & \\
\hline FL & SRWMD & -011011002 & - & -011011002 & Madison & 302450 & 831752 & $5 / 11 / 2010$ & 51 & \\
\hline FL & SRWMD & -011035001 & 302100083171001 & -011035001 & Madison & 302059 & 831711 & $5 / 11 / 2010$ & 56 & \\
\hline
\end{tabular}


Table 1. Water-level measurements in Florida and parts of Georgia, South Carolina, and Alabama, May-June 2010.—Continued

[NGVD 29, National Geodetic Vertical Datum of 1929. Abbreviations for agency database: ACEPD, Alachua County Environmental Protection Department; FGS, Florida Geological Survey; GSA, Geological Survey of Alabama; NWFWMD, Northwest Florida Water Management District; SFWMD, South Florida Water Management District; SRWMD, Suwannee River Water Management District; USGSALWSC, U.S. Geological Survey Alabama Water Science Center; USGS-FLWSC, U.S. Geological Survey Florida Water Science Center; GAWSC, U.S. Geological Survey Georgia Water Science Center; SCWSC, U.S. Geological Survey South Carolina Water Science Center. Abbreviations for Station names: BLVD., boulevard; CO, county; CR, county road; E, east; HS, high school; IFAS, Institute of Food and Agricultural Services; LK, lake; MW, monitoring well; N, north; NE, northeast; NE, northeast; NF, national forest; NW, northwest; PK, park; R., river; RD., road; SE, southeast; SE, southeast; SR, state road; ST., street; TWR, tower; UFA, Upper Floridan aquifer; - - not available; \#, number; “, inches]

\begin{tabular}{|c|c|c|c|c|c|c|c|c|c|c|}
\hline State & $\begin{array}{c}\text { Agency } \\
\text { database }\end{array}$ & Station name & $\begin{array}{c}\text { USGS site } \\
\text { identification } \\
\text { number }\end{array}$ & $\begin{array}{l}\text { Local agency } \\
\text { unique site } \\
\text { identifier }\end{array}$ & County & $\begin{array}{l}\text { Latitude } \\
\text { (degree } \\
\text { minutes } \\
\text { and } \\
\text { seconds) } \\
\text { [DDMMSS] }\end{array}$ & $\begin{array}{l}\text { Longitude } \\
\text { (degree } \\
\text { minutes } \\
\text { and } \\
\text { seconds) } \\
\text { [DDMMSS] }\end{array}$ & $\begin{array}{c}\text { Date of } \\
\text { measure- } \\
\text { ment }\end{array}$ & $\begin{array}{c}\text { Water } \\
\text { level } \\
\text { altitude } \\
\text { (feet } \\
\text { above } \\
\text { NGVD 29) }\end{array}$ & Remarks \\
\hline FL & SRWMD & -011125001 & - & -011125001 & Suwannee & 302203 & 831034 & $5 / 25 / 2010$ & 33 & \\
\hline FL & SRWMD & -011219001 & - & -011219001 & Suwannee & 302304 & 830955 & $5 / 25 / 2010$ & 36 & \\
\hline FL & SRWMD & -011232006 & - & -011232006 & Suwannee & 302129 & 830756 & $5 / 12 / 2010$ & 37 & \\
\hline FL & SRWMD & -011323001 & - & -011323001 & Suwannee & 302334 & 825912 & $5 / 25 / 2010$ & 42 & \\
\hline FL & SRWMD & -011420001 & 302334082560201 & -011420001 & Suwannee & 302334 & 825602 & $5 / 24 / 2010$ & 41 & \\
\hline FL & SRWMD & -011420006 & - & -011420006 & Suwannee & 302323 & 825621 & $5 / 3 / 2010$ & 43 & \\
\hline FL & SRWMD & -011511001 & - & -011511001 & Hamilton & 302442 & 824700 & $5 / 12 / 2010$ & 52 & \\
\hline FL & SRWMD & -011521001 & 302323082493501 & -011521001 & Hamilton & 302323 & 824935 & $5 / 13 / 2010$ & 49 & \\
\hline FL & SRWMD & -011534001 & 302127082475801 & -011534001 & Hamilton & 302125 & 824758 & $5 / 12 / 2010$ & 52 & \\
\hline FL & SRWMD & -011535004 & - & -011535004 & Hamilton & 302108 & 824653 & $5 / 13 / 2010$ & 52 & \\
\hline FL & SRWMD & -011627001 & 302208082420101 & -011627001 & Hamilton & 302209 & 824200 & $5 / 13 / 2010$ & 53 & \\
\hline FL & SRWMD & -011727001 & 302243082360201 & -011727001 & Columbia & 302250 & 823622 & $5 / 6 / 2010$ & 52 & \\
\hline FL & SRWMD & -011728001 & - & -011728001 & Columbia & 302232 & 823721 & $5 / 20 / 2010$ & 53 & \\
\hline FL & SRWMD & -020433001 & - & -020433001 & Jefferson & 301617 & 835538 & $5 / 18 / 2010$ & 29 & \\
\hline FL & SRWMD & -020731002 & 301555083393985 & -020731002 & Taylor & 301548 & 833940 & $5 / 13 / 2010$ & 63 & \\
\hline FL & SRWMD & -020802001 & 302049083293501 & -020802001 & Madison & 302049 & 832935 & $5 / 10 / 2010$ & 91 & \\
\hline FL & SRWMD & -020828001 & - & -020828001 & Taylor & 301722 & 833210 & $5 / 17 / 2010$ & 89 & \\
\hline FL & SRWMD & -021104001 & - & -021104001 & Madison & 302037 & 831326 & $5 / 11 / 2010$ & 36 & \\
\hline FL & SRWMD & -021111001 & 301959083115001 & -021111001 & Suwannee & 301960 & 831150 & $5 / 12 / 2010$ & 33 & \\
\hline FL & SRWMD & -021127001 & - & -021127001 & Suwannee & 301648 & 831251 & $5 / 12 / 2010$ & 38 & \\
\hline FL & SRWMD & -021217001 & 301839083084901 & -021217001 & Suwannee & 301839 & 830849 & $5 / 12 / 2010$ & 37 & \\
\hline FL & SRWMD & -021231001 & - & -021231001 & Suwannee & 301630 & 830912 & $5 / 12 / 2010$ & 37 & \\
\hline FL & SRWMD & -021322008 & - & -021322008 & Suwannee & 301727 & 830026 & $5 / 13 / 2010$ & 40 & \\
\hline FL & SRWMD & -021335001 & - & -021335001 & Suwannee & 301613 & 825910 & $5 / 13 / 2010$ & 40 & \\
\hline
\end{tabular}


Table 1. Water-level measurements in Florida and parts of Georgia, South Carolina, and Alabama, May-June 2010.—Continued

[NGVD 29, National Geodetic Vertical Datum of 1929. Abbreviations for agency database: ACEPD, Alachua County Environmental Protection Department; FGS, Florida Geological Survey; GSA, Geological Survey of Alabama; NWFWMD, Northwest Florida Water Management District; SFWMD, South Florida Water Management District; SRWMD, Suwannee River Water Management District; USGSALWSC, U.S. Geological Survey Alabama Water Science Center; USGS-FLWSC, U.S. Geological Survey Florida Water Science Center; GAWSC, U.S. Geological Survey Georgia Water Science Center; SCWSC, U.S. Geological Survey South Carolina Water Science Center. Abbreviations for Station names: BLVD., boulevard; CO, county; CR, county road; E, east; HS, high school; IFAS, Institute of Food and Agricultural Services; LK, lake; MW, monitoring well; N, north; NE, northeast; NE, northeast; NF, national forest; NW, northwest; PK, park; R., river; RD., road; SE, southeast; SE, southeast; SR, state road; ST., street; TWR, tower; UFA, Upper Floridan aquifer; —, not available; \#, number; “, inches]

\begin{tabular}{|c|c|c|c|c|c|c|c|c|c|c|}
\hline State & $\begin{array}{c}\text { Agency } \\
\text { database }\end{array}$ & Station name & $\begin{array}{c}\text { USGS site } \\
\text { identification } \\
\text { number }\end{array}$ & $\begin{array}{l}\text { Local agency } \\
\text { unique site } \\
\text { identifier }\end{array}$ & County & $\begin{array}{l}\text { Latitude } \\
\text { (degree } \\
\text { minutes } \\
\text { and } \\
\text { seconds) } \\
\text { [DDMMSS }\end{array}$ & $\begin{array}{l}\text { Longitude } \\
\text { (degree } \\
\text { minutes } \\
\text { and } \\
\text { seconds) } \\
\text { [DDMMSS] }\end{array}$ & $\begin{array}{c}\text { Date of } \\
\text { measure- } \\
\text { ment }\end{array}$ & $\begin{array}{c}\text { Water } \\
\text { level } \\
\text { altitude } \\
\text { (feet } \\
\text { above } \\
\text { NGVD 29) }\end{array}$ & Remarks \\
\hline FL & SRWMD & -021516001 & 301909082490985 & -021516001 & Suwannee & 301911 & 824909 & $5 / 13 / 2010$ & 52 & \\
\hline FL & SRWMD & -021624001 & 301822082393901 & -021624001 & Columbia & 301821 & 823938 & $5 / 20 / 2010$ & 54 & \\
\hline FL & SRWMD & -021711003 & 301933082350522 & -021711003 & Columbia & 301935 & 823509 & $5 / 17 / 2010$ & 54 & \\
\hline FL & SRWMD & -021805001 & 302052082312401 & -021805001 & Columbia & 302037 & 823135 & $5 / 17 / 2010$ & 52 & \\
\hline FL & SRWMD & -021902001 & 302115082232201 & -021902001 & Baker & 302112 & 822335 & $5 / 6 / 2010$ & 53 & \\
\hline FL & SRWMD & -021930001 & 301702082271501 & -021930001 & Baker & 301705 & 822717 & $5 / 6 / 2010$ & 55 & \\
\hline FL & SRWMD & -021934001 & 301635082234001 & -021934001 & Baker & 301635 & 822340 & $5 / 6 / 2010$ & 54 & \\
\hline FL & SRWMD & -030328001 & 301118084014001 & -030328001 & Jefferson & 301117 & 840141 & $5 / 18 / 2010$ & 9 & \\
\hline FL & SRWMD & -030424003 & - & -030424003 & Taylor & 301241 & 835235 & $5 / 18 / 2010$ & 28 & \\
\hline FL & SRWMD & -030730001 & 301145083401201 & -030730001 & Taylor & 301145 & 834012 & $5 / 13 / 2010$ & 43 & \\
\hline FL & SRWMD & -030833001 & - & -030833001 & Taylor & 301113 & 833140 & $5 / 17 / 2010$ & 87 & \\
\hline FL & SRWMD & -031012001 & 301405083163001 & -031012001 & Lafayette & 301406 & 831637 & $5 / 12 / 2010$ & 64 & \\
\hline FL & SRWMD & -031103002 & - & -031103002 & Suwannee & 301504 & 831156 & $5 / 25 / 2010$ & 38 & \\
\hline FL & SRWMD & -031105006 & - & -031105006 & Suwannee & 301455 & 831418 & $5 / 12 / 2010$ & 29 & \\
\hline FL & SRWMD & -031134001 & - & -031134001 & Suwannee & 301042 & 831223 & $5 / 25 / 2010$ & 29 & \\
\hline FL & SRWMD & -031219001 & - & -031219001 & Suwannee & 301259 & 830854 & $5 / 12 / 2010$ & 32 & \\
\hline FL & SRWMD & -031232001 & - & -031232001 & Suwannee & 301034 & 830846 & $5 / 14 / 2010$ & 31 & \\
\hline FL & SRWMD & -031232002 & - & -031232002 & Suwannee & 301037 & 830834 & $5 / 4 / 2010$ & 31 & \\
\hline FL & SRWMD & -031305005 & - & -031305005 & Suwannee & 301514 & 830213 & $5 / 4 / 2010$ & 39 & \\
\hline FL & SRWMD & -031326001 & 301149082593801 & -031326001 & Suwannee & 301149 & 825938 & $5 / 25 / 2010$ & 32 & \\
\hline FL & SRWMD & -031335002 & - & -031335002 & Suwannee & 301116 & 825917 & $5 / 3 / 2010$ & 32 & \\
\hline FL & SRWMD & -031601003 & - & -031601003 & Columbia & 301534 & 823956 & $5 / 20 / 2010$ & 55 & \\
\hline FL & SRWMD & -031734011 & - & -031734011 & Columbia & 301104 & 823537 & $5 / 19 / 2010$ & 53 & \\
\hline FL & SRWMD & -031807001 & - & -031807001 & Columbia & 301434 & 823241 & $5 / 17 / 2010$ & 56 & \\
\hline
\end{tabular}


Table 1. Water-level measurements in Florida and parts of Georgia, South Carolina, and Alabama, May-June 2010.—Continued

[NGVD 29, National Geodetic Vertical Datum of 1929. Abbreviations for agency database: ACEPD, Alachua County Environmental Protection Department; FGS, Florida Geological Survey; GSA, Geological Survey of Alabama; NWFWMD, Northwest Florida Water Management District; SFWMD, South Florida Water Management District; SRWMD, Suwannee River Water Management District; USGSALWSC, U.S. Geological Survey Alabama Water Science Center; USGS-FLWSC, U.S. Geological Survey Florida Water Science Center; GAWSC, U.S. Geological Survey Georgia Water Science Center; SCWSC, U.S. Geological Survey South Carolina Water Science Center. Abbreviations for Station names: BLVD., boulevard; CO, county; CR, county road; E, east; HS, high school; IFAS, Institute of Food and Agricultural Services; LK, lake; MW, monitoring well; N, north; NE, northeast; NE, northeast; NF, national forest; NW, northwest; PK, park; R., river; RD., road; SE, southeast; SE, southeast; SR, state road; ST., street; TWR, tower; UFA, Upper Floridan aquifer; - - not available; \#, number; “, inches]

\begin{tabular}{|c|c|c|c|c|c|c|c|c|c|c|}
\hline State & $\begin{array}{c}\text { Agency } \\
\text { database }\end{array}$ & Station name & $\begin{array}{c}\text { USGS site } \\
\text { identification } \\
\text { number }\end{array}$ & $\begin{array}{c}\text { Local agency } \\
\text { unique site } \\
\text { identifier }\end{array}$ & County & $\begin{array}{l}\text { Latitude } \\
\text { (degree } \\
\text { minutes } \\
\text { and } \\
\text { seconds) } \\
\text { [DDMMS] }\end{array}$ & $\begin{array}{l}\text { Longitude } \\
\text { (degree } \\
\text { minutes } \\
\text { and } \\
\text { seconds) } \\
\text { ] [DDMMSS] }\end{array}$ & $\begin{array}{c}\text { Date of } \\
\text { measure- } \\
\text { ment }\end{array}$ & $\begin{array}{c}\text { Water } \\
\text { level } \\
\text { altitude } \\
\text { (feet } \\
\text { above } \\
\text { NGVD 29) }\end{array}$ & Remarks \\
\hline FL & SRWMD & -031923004 & - & -031923004 & Baker & 301246 & 822329 & $5 / 6 / 2010$ & 55 & \\
\hline FL & SRWMD & -040407001 & 300851083580801 & -040407001 & Taylor & 300849 & 835808 & $5 / 18 / 2010$ & 2 & \\
\hline FL & SRWMD & -040736005 & 300530083342085 & -040736005 & Taylor & 300540 & 833418 & $5 / 17 / 2010$ & 36 & \\
\hline FL & SRWMD & -041014001 & 300823083175901 & -041014001 & Lafayette & 300825 & 831756 & $5 / 11 / 2010$ & 47 & \\
\hline FL & SRWMD & -041112005 & - & -041112005 & Suwannee & 300905 & 831010 & $5 / 14 / 2010$ & 28 & \\
\hline FL & SRWMD & -041123001 & 300718083112301 & -041123001 & Suwannee & 300719 & 831122 & $5 / 25 / 2010$ & 25 & \\
\hline FL & SRWMD & -041131002 & - & -041131002 & Lafayette & 300513 & 831527 & $5 / 11 / 2010$ & 35 & \\
\hline FL & SRWMD & -041133001 & - & -041133001 & Lafayette & 300511 & 831323 & $5 / 11 / 2010$ & 22 & \\
\hline FL & SRWMD & -041211001 & - & -041211001 & Suwannee & 300843 & 830501 & $5 / 12 / 2010$ & 24 & \\
\hline FL & SRWMD & -041227001 & - & -041227001 & Suwannee & 300644 & 830647 & $5 / 4 / 2010$ & 22 & \\
\hline FL & SRWMD & -041329001 & - & -041329001 & Suwannee & 300629 & 830239 & $5 / 24 / 2010$ & 25 & \\
\hline FL & SRWMD & -041402002 & - & -041402002 & Suwannee & 300954 & 825319 & $5 / 3 / 2010$ & 31 & \\
\hline FL & SRWMD & -041523001 & - & -041523001 & Columbia & 300752 & 824738 & $5 / 20 / 2010$ & 31 & \\
\hline FL & SRWMD & -041625001 & - & -041625001 & Columbia & 300706 & 824019 & $5 / 19 / 2010$ & 34 & \\
\hline FL & SRWMD & -041705001 & 301031082381001 & -041705001 & Columbia & 301031 & 823810 & $5 / 17 / 2010$ & 46 & \\
\hline FL & SRWMD & -041827002 & - & -041827002 & Columbia & 300635 & 822948 & $5 / 17 / 2010$ & 50 & \\
\hline FL & SRWMD & -042236001 & - & -042236001 & Bradford & 300626 & 820259 & $5 / 24 / 2010$ & 56 & \\
\hline FL & SRWMD & -050615001 & 300224083430501 & -050615001 & Taylor & 300225 & 834305 & $5 / 18 / 2010$ & 13 & \\
\hline FL & SRWMD & -050615002 & - & -050615002 & Taylor & 300227 & 834303 & $5 / 18 / 2010$ & 13 & \\
\hline FL & SRWMD & -050701001 & 300447083343001 & -050701001 & Taylor & 300448 & 833418 & $5 / 17 / 2010$ & 35 & \\
\hline FL & SRWMD & -050928004 & 300131083260485 & -050928004 & Taylor & 300130 & 832604 & $5 / 11 / 2010$ & 71 & \\
\hline FL & SRWMD & -051002001 & 300433083173401 & -051002001 & Lafayette & 300434 & 831734 & $5 / 11 / 2010$ & 76 & \\
\hline FL & SRWMD & -051004001 & - & -051004001 & Lafayette & 300422 & 831908 & $5 / 11 / 2010$ & 85 & \\
\hline FL & SRWMD & -051201007 & - & -051201007 & Suwannee & 300507 & 830431 & $5 / 12 / 2010$ & 19 & \\
\hline
\end{tabular}


Table 1. Water-level measurements in Florida and parts of Georgia, South Carolina, and Alabama, May-June 2010.-Continued

[NGVD 29, National Geodetic Vertical Datum of 1929. Abbreviations for agency database: ACEPD, Alachua County Environmental Protection Department; FGS, Florida Geological Survey; GSA, Geological Survey of Alabama; NWFWMD, Northwest Florida Water Management District; SFWMD, South Florida Water Management District; SRWMD, Suwannee River Water Management District; USGSALWSC, U.S. Geological Survey Alabama Water Science Center; USGS-FLWSC, U.S. Geological Survey Florida Water Science Center; GAWSC, U.S. Geological Survey Georgia Water Science Center; SCWSC, U.S. Geological Survey South Carolina Water Science Center. Abbreviations for Station names: BLVD., boulevard; CO, county; CR, county road; E, east; HS, high school; IFAS, Institute of Food and Agricultural Services; LK, lake; MW, monitoring well; N, north; NE, northeast; NE, northeast; NF, national forest; NW, northwest; PK, park; R., river; RD., road; SE, southeast; SE, southeast; SR, state road; ST., street; TWR, tower; UFA, Upper Floridan aquifer; —, not available; \#, number; “, inches]

\begin{tabular}{|c|c|c|c|c|c|c|c|c|c|c|}
\hline State & $\begin{array}{c}\text { Agency } \\
\text { database }\end{array}$ & Station name & $\begin{array}{c}\text { USGS site } \\
\text { identification } \\
\text { number }\end{array}$ & $\begin{array}{l}\text { Local agency } \\
\text { unique site } \\
\text { identifier }\end{array}$ & County & $\begin{array}{l}\text { Latitude } \\
\text { (degree } \\
\text { minutes } \\
\text { and } \\
\text { seconds) } \\
\text { [DDMMSS }\end{array}$ & $\begin{array}{l}\text { Longitude } \\
\text { (degree } \\
\text { minutes } \\
\text { and } \\
\text { seconds) } \\
\text { [DDMMSS] }\end{array}$ & $\begin{array}{c}\text { Date of } \\
\text { measure- } \\
\text { ment }\end{array}$ & $\begin{array}{c}\text { Water } \\
\text { level } \\
\text { altitude } \\
\text { (feet } \\
\text { above } \\
\text { NGVD 29) }\end{array}$ & Remarks \\
\hline FL & SRWMD & -051208001 & - & -051208001 & Lafayette & 300406 & 830817 & $5 / 10 / 2010$ & 31 & \\
\hline FL & SRWMD & -051214008 & - & -051214008 & Lafayette & 300250 & 830454 & $5 / 10 / 2010$ & 25 & \\
\hline FL & SRWMD & -051218002 & - & -051218002 & Lafayette & 300309 & 830930 & $5 / 10 / 2010$ & 49 & \\
\hline FL & SRWMD & -051311001 & 300400082585001 & -051311001 & Suwannee & 300354 & 825842 & $5 / 12 / 2010$ & 21 & \\
\hline FL & SRWMD & -051328002 & - & -051328002 & Lafayette & 300130 & 830126 & $5 / 11 / 2010$ & 20 & \\
\hline FL & SRWMD & -051331002 & - & -051331002 & Lafayette & 295957 & 830327 & $5 / 27 / 2010$ & 43 & \\
\hline FL & SRWMD & -051334013 & - & -051334013 & Lafayette & 300010 & 825943 & $5 / 12 / 2010$ & 19 & \\
\hline FL & SRWMD & -051405002 & - & -051405002 & Suwannee & 300435 & 825641 & $5 / 3 / 2010$ & 25 & \\
\hline FL & SRWMD & -051428004 & 300117082554285 & -051428004 & Suwannee & 300116 & 825542 & $5 / 24 / 2010$ & 24 & \\
\hline FL & SRWMD & -051511002 & - & -051511002 & Columbia & 300335 & 824730 & $5 / 20 / 2010$ & 30 & \\
\hline FL & SRWMD & -051610001 & 300409082415001 & -051610001 & Columbia & 300417 & 824147 & $5 / 19 / 2010$ & 29 & \\
\hline FL & SRWMD & -051610006 & - & -051610006 & Columbia & 300404 & 824204 & $5 / 19 / 2010$ & 29 & \\
\hline FL & SRWMD & -051621002 & - & -051621002 & Columbia & 300242 & 824317 & $5 / 26 / 2010$ & 28 & \\
\hline FL & SRWMD & -051734001 & 300019082355401 & -051734001 & Columbia & 300012 & 823552 & $5 / 19 / 2010$ & 33 & \\
\hline FL & SRWMD & -051819001 & 300221082332601 & -051819001 & Columbia & 300221 & 823324 & $5 / 19 / 2010$ & 40 & \\
\hline FL & SRWMD & -051922001 & - & -051922001 & Union & 300243 & 822330 & $5 / 19 / 2010$ & 56 & \\
\hline FL & SRWMD & -060705001 & 295910083385101 & -060705001 & Taylor & 295907 & 833843 & $5 / 19 / 2010$ & 24 & \\
\hline FL & SRWMD & -061005001 & - & -061005001 & Lafayette & 295903 & 832040 & $5 / 11 / 2010$ & 69 & \\
\hline FL & SRWMD & -061025003 & - & -061025003 & Lafayette & 295540 & 831645 & $5 / 10 / 2010$ & 51 & \\
\hline FL & SRWMD & -061114001 & 295802083121001 & -061114001 & Lafayette & 295752 & 831210 & $5 / 10 / 2010$ & 69 & \\
\hline FL & SRWMD & -061225004 & - & -061225004 & Lafayette & 295538 & 830424 & $5 / 25 / 2010$ & 64 & \\
\hline FL & SRWMD & -061301007 & - & -061301007 & Suwannee & 295947 & 825758 & $5 / 11 / 2010$ & 16 & \\
\hline FL & SRWMD & -061327002 & - & -061327002 & Lafayette & 295532 & 830056 & $5 / 25 / 2010$ & 63 & \\
\hline FL & SRWMD & -061401003 & - & -061401003 & Suwannee & 295950 & 825145 & $5 / 3 / 2010$ & 28 & \\
\hline
\end{tabular}


Table 1. Water-level measurements in Florida and parts of Georgia, South Carolina, and Alabama, May-June 2010.—Continued

[NGVD 29, National Geodetic Vertical Datum of 1929. Abbreviations for agency database: ACEPD, Alachua County Environmental Protection Department; FGS, Florida Geological Survey; GSA, Geological Survey of Alabama; NWFWMD, Northwest Florida Water Management District; SFWMD, South Florida Water Management District; SRWMD, Suwannee River Water Management District; USGSALWSC, U.S. Geological Survey Alabama Water Science Center; USGS-FLWSC, U.S. Geological Survey Florida Water Science Center; GAWSC, U.S. Geological Survey Georgia Water Science Center; SCWSC, U.S. Geological Survey South Carolina Water Science Center. Abbreviations for Station names: BLVD., boulevard; CO, county; CR, county road; E, east; HS, high school; IFAS, Institute of Food and Agricultural Services; LK, lake; MW, monitoring well; N, north; NE, northeast; NE, northeast; NF, national forest; NW, northwest; PK, park; R., river; RD., road; SE, southeast; SE, southeast; SR, state road; ST., street; TWR, tower; UFA, Upper Floridan aquifer; - , not available; \#, number; “, inches]

\begin{tabular}{|c|c|c|c|c|c|c|c|c|c|c|}
\hline State & $\begin{array}{c}\text { Agency } \\
\text { database }\end{array}$ & Station name & $\begin{array}{c}\text { USGS site } \\
\text { identification } \\
\text { number }\end{array}$ & $\begin{array}{c}\text { Local agency } \\
\text { unique site } \\
\text { identifier }\end{array}$ & County & $\begin{array}{l}\text { Latitude } \\
\text { (degree } \\
\text { minutes } \\
\text { and } \\
\text { seconds) } \\
\text { [DDMMS] }\end{array}$ & $\begin{array}{l}\text { Longitude } \\
\text { (degree } \\
\text { minutes } \\
\text { and } \\
\text { seconds) } \\
\text { ] [DDMMSS] }\end{array}$ & $\begin{array}{c}\text { Date of } \\
\text { measure- } \\
\text { ment }\end{array}$ & $\begin{array}{c}\text { Water } \\
\text { level } \\
\text { altitude } \\
\text { (feet } \\
\text { above } \\
\text { NGVD 29) }\end{array}$ & Remarks \\
\hline FL & SRWMD & -061434006 & - & -061434006 & Suwannee & 295531 & 825416 & $5 / 5 / 2010$ & 18 & \\
\hline FL & SRWMD & -061521005 & - & -061521005 & Suwannee & 295659 & 824935 & $5 / 5 / 2010$ & 16 & \\
\hline FL & SRWMD & -061527001 & - & -061527001 & Suwannee & 295615 & 824754 & $5 / 24 / 2010$ & 10 & \\
\hline FL & SRWMD & -061610001 & - & -061610001 & Columbia & 295903 & 824236 & $5 / 20 / 2010$ & 27 & \\
\hline FL & SRWMD & -061618003 & - & -061618003 & Columbia & 295812 & 824506 & $5 / 14 / 2010$ & 24 & \\
\hline FL & SRWMD & -061629001 & 295618082440901 & -061629001 & Columbia & 295620 & 824410 & $5 / 24 / 2010$ & 22 & \\
\hline FL & SRWMD & -061734001 & - & -061734001 & Columbia & 295514 & 823629 & $5 / 19 / 2010$ & 34 & \\
\hline FL & SRWMD & -061804001 & - & -061804001 & Union & 295931 & 823116 & $5 / 19 / 2010$ & 40 & \\
\hline FL & SRWMD & -061920001 & - & -061920001 & Union & 295701 & 822531 & $5 / 18 / 2010$ & 51 & \\
\hline FL & SRWMD & -061932026 & - & -061932026 & Union & 295541 & 822529 & $5 / 18 / 2010$ & 51 & \\
\hline FL & SRWMD & -071203001 & - & -071203001 & Lafayette & 295422 & 830659 & $5 / 25 / 2010$ & 63 & \\
\hline FL & SRWMD & -071213003 & - & -071213003 & Lafayette & 295256 & 830422 & $5 / 25 / 2010$ & 63 & \\
\hline FL & SRWMD & -071216001 & - & -071216001 & Lafayette & 295205 & 830719 & $5 / 25 / 2010$ & 62 & \\
\hline FL & SRWMD & -071234001 & - & -071234001 & Lafayette & 295010 & 830619 & $5 / 25 / 2010$ & 61 & \\
\hline FL & SRWMD & -071310002 & - & -071310002 & Lafayette & 295324 & 830047 & $5 / 25 / 2010$ & 63 & \\
\hline FL & SRWMD & -071321001 & - & -071321001 & Lafayette & 295121 & 830120 & $5 / 25 / 2010$ & 62 & \\
\hline FL & SRWMD & -071331001 & - & -071331001 & Lafayette & 295019 & 830338 & $5 / 25 / 2010$ & 62 & \\
\hline FL & SRWMD & -071333002 & - & -071333002 & Lafayette & 294939 & 830121 & $5 / 25 / 2010$ & 60 & \\
\hline FL & SRWMD & -071515001 & 295014082482501 & -071515001 & Gilchrist & 295215 & 824824 & $5 / 17 / 2010$ & 66 & \\
\hline FL & SRWMD & -071528001 & 295057082483485 & -071528001 & Gilchrist & 295057 & 824830 & $5 / 11 / 2010$ & 67 & \\
\hline FL & SRWMD & -071927008 & - & -071927008 & Alachua & 295130 & 822429 & $5 / 18 / 2010$ & 43 & \\
\hline FL & SRWMD & -072002001 & - & -072002001 & Bradford & 295456 & 821705 & $5 / 20 / 2010$ & 58 & \\
\hline FL & SRWMD & -072215001 & 295257082045701 & -072215001 & Bradford & 295257 & 820456 & $5 / 18 / 2010$ & 81 & \\
\hline FL & SRWMD & -080907002 & - & -080907002 & Taylor & 294752 & 832803 & $5 / 19 / 2010$ & 32 & \\
\hline
\end{tabular}


Table 1. Water-level measurements in Florida and parts of Georgia, South Carolina, and Alabama, May-June 2010.—Continued

[NGVD 29, National Geodetic Vertical Datum of 1929. Abbreviations for agency database: ACEPD, Alachua County Environmental Protection Department; FGS, Florida Geological Survey; GSA, Geological Survey of Alabama; NWFWMD, Northwest Florida Water Management District; SFWMD, South Florida Water Management District; SRWMD, Suwannee River Water Management District; USGSALWSC, U.S. Geological Survey Alabama Water Science Center; USGS-FLWSC, U.S. Geological Survey Florida Water Science Center; GAWSC, U.S. Geological Survey Georgia Water Science Center; SCWSC, U.S. Geological Survey South Carolina Water Science Center. Abbreviations for Station names: BLVD., boulevard; CO, county; CR, county road; E, east; HS, high school; IFAS, Institute of Food and Agricultural Services; LK, lake; MW, monitoring well; N, north; NE, northeast; NE, northeast; NF, national forest; NW, northwest; PK, park; R., river; RD., road; SE, southeast; SE, southeast; SR, state road; ST., street; TWR, tower; UFA, Upper Floridan aquifer; - , not available; \#, number; “, inches]

\begin{tabular}{|c|c|c|c|c|c|c|c|c|c|c|}
\hline State & $\begin{array}{c}\text { Agency } \\
\text { database }\end{array}$ & Station name & $\begin{array}{c}\text { USGS site } \\
\text { identification } \\
\text { number }\end{array}$ & $\begin{array}{l}\text { Local agency } \\
\text { unique site } \\
\text { identifier }\end{array}$ & County & $\begin{array}{l}\text { Latitude } \\
\text { (degree } \\
\text { minutes } \\
\text { and } \\
\text { seconds) } \\
\text { [DDMMSS] }\end{array}$ & $\begin{array}{l}\text { Longitude } \\
\text { (degree } \\
\text { minutes } \\
\text { and } \\
\text { seconds) } \\
\text { [DDMMSS] }\end{array}$ & $\begin{array}{c}\text { Date of } \\
\text { measure- } \\
\text { ment }\end{array}$ & $\begin{array}{c}\text { Water } \\
\text { level } \\
\text { altitude } \\
\text { (feet } \\
\text { above } \\
\text { NGVD 29) }\end{array}$ & Remarks \\
\hline FL & SRWMD & -080907003 & 294742083275985 & -080907003 & Taylor & 294741 & 832800 & $5 / 19 / 2010$ & 29 & \\
\hline FL & SRWMD & -081132001 & 294458083142801 & -081132001 & Dixie & 294456 & 831428 & $5 / 10 / 2010$ & 38 & \\
\hline FL & SRWMD & -081313005 & - & -081313005 & Dixie & 294656 & 825808 & $5 / 10 / 2010$ & 21 & \\
\hline FL & SRWMD & -081416001 & 294743082543901 & -081416001 & Gilchrist & 294744 & 825438 & $5 / 17 / 2010$ & 10 & \\
\hline FL & SRWMD & -081618001 & 294721082443001 & -081618001 & Gilchrist & 294722 & 824429 & $5 / 11 / 2010$ & 33 & \\
\hline FL & SRWMD & -081806005 & - & -081806005 & Alachua & 294941 & 823241 & $5 / 27 / 2010$ & 33 & \\
\hline FL & SRWMD & -081823001 & - & -081823001 & Alachua & 294628 & 822904 & $5 / 19 / 2010$ & 35 & \\
\hline FL & SRWMD & -082003001 & 294923082174501 & -082003001 & Alachua & 294924 & 821744 & $5 / 19 / 2010$ & 54 & \\
\hline FL & SRWMD & -082202001 & - & -082202001 & Bradford & 294919 & 820447 & $5 / 6 / 2010$ & 77 & \\
\hline FL & SRWMD & -090914003 & - & -090914003 & Taylor & 294142 & 832317 & $5 / 19 / 2010$ & 20 & \\
\hline FL & SRWMD & -091011004 & - & -091011004 & Dixie & 294231 & 831742 & $5 / 4 / 2010$ & 29 & \\
\hline FL & SRWMD & -091212003 & - & -091212003 & Dixie & 294311 & 830413 & $5 / 10 / 2010$ & 44 & \\
\hline FL & SRWMD & -091231001 & - & -091231001 & Dixie & 293859 & 830936 & $5 / 4 / 2010$ & 38 & \\
\hline FL & SRWMD & -091420001 & 294135082553401 & -091420001 & Gilchrist & 294135 & 825531 & $5 / 26 / 2010$ & 7 & \\
\hline FL & SRWMD & -091504001 & 294400082491385 & -091504001 & Gilchrist & 294358 & 824910 & $5 / 11 / 2010$ & 81 & \\
\hline FL & SRWMD & -091607001 & 294330082445001 & -091607001 & Gilchrist & 294329 & 824448 & $5 / 20 / 2010$ & 44 & \\
\hline FL & SRWMD & -091704001 & 294428082362901 & -091704001 & Alachua & 294429 & 823628 & $5 / 19 / 2010$ & 34 & \\
\hline FL & SRWMD & -091822001 & - & -091822001 & Alachua & 294154 & 822935 & $5 / 19 / 2010$ & 31 & \\
\hline FL & SRWMD & -101210001 & 293731083061801 & -101210001 & Dixie & 293730 & 830617 & $5 / 10 / 2010$ & 33 & \\
\hline FL & SRWMD & -101303003 & - & -101303003 & Dixie & 293826 & 830046 & $5 / 5 / 2010$ & 13 & \\
\hline FL & SRWMD & -101336025 & - & -101336025 & Dixie & 293426 & 825810 & $5 / 5 / 2010$ & 9 & \\
\hline FL & SRWMD & -101429011 & - & -101429011 & Levy & 293507 & 825623 & $5 / 18 / 2010$ & 5 & \\
\hline FL & SRWMD & -101429020 & - & -101429020 & Levy & 293514 & 825541 & $5 / 18 / 2010$ & 6 & \\
\hline FL & SRWMD & -101430002 & - & -101430002 & Dixie & 293513 & 825649 & $5 / 10 / 2010$ & 4 & \\
\hline
\end{tabular}


Table 1. Water-level measurements in Florida and parts of Georgia, South Carolina, and Alabama, May-June 2010.—Continued

[NGVD 29, National Geodetic Vertical Datum of 1929. Abbreviations for agency database: ACEPD, Alachua County Environmental Protection Department; FGS, Florida Geological Survey; GSA, Geological Survey of Alabama; NWFWMD, Northwest Florida Water Management District; SFWMD, South Florida Water Management District; SRWMD, Suwannee River Water Management District; USGSALWSC, U.S. Geological Survey Alabama Water Science Center; USGS-FLWSC, U.S. Geological Survey Florida Water Science Center; GAWSC, U.S. Geological Survey Georgia Water Science Center; SCWSC, U.S. Geological Survey South Carolina Water Science Center. Abbreviations for Station names: BLVD., boulevard; CO, county; CR, county road; E, east; HS, high school; IFAS, Institute of Food and Agricultural Services; LK, lake; MW, monitoring well; N, north; NE, northeast; NE, northeast; NF, national forest; NW, northwest; PK, park; R., river; RD., road; SE, southeast; SE, southeast; SR, state road; ST., street; TWR, tower; UFA, Upper Floridan aquifer; - , not available; \#, number; “, inches]

\begin{tabular}{|c|c|c|c|c|c|c|c|c|c|c|}
\hline State & $\begin{array}{c}\text { Agency } \\
\text { database }\end{array}$ & Station name & $\begin{array}{c}\text { USGS site } \\
\text { identification } \\
\text { number }\end{array}$ & $\begin{array}{l}\text { Local agency } \\
\text { unique site } \\
\text { identifier }\end{array}$ & County & $\begin{array}{l}\text { Latitude } \\
\text { (degree } \\
\text { minutes } \\
\text { and } \\
\text { seconds) } \\
\text { [DDMMSS] }\end{array}$ & $\begin{array}{l}\text { Longitude } \\
\text { (degree } \\
\text { minutes } \\
\text { and } \\
\text { seconds) } \\
\text { [DDMMSS] }\end{array}$ & $\begin{array}{c}\text { Date of } \\
\text { measure- } \\
\text { ment }\end{array}$ & $\begin{array}{c}\text { Water } \\
\text { level } \\
\text { altitude } \\
\text { (feet } \\
\text { above } \\
\text { NGVD 29) }\end{array}$ & Remarks \\
\hline FL & SRWMD & -101506003 & - & -101506003 & Gilchrist & 293832 & 825117 & $5 / 20 / 2010$ & 9 & \\
\hline FL & SRWMD & -101516017 & - & -101516017 & Gilchrist & 293639 & 824860 & $5 / 20 / 2010$ & 11 & \\
\hline FL & SRWMD & -101601002 & 293827082395401 & -101601002 & Gilchrist & 293828 & 823950 & $5 / 7 / 2010$ & 39 & \\
\hline FL & SRWMD & -101634001 & 293414082415285 & -101634001 & Gilchrist & 293413 & 824149 & $5 / 7 / 2010$ & 62 & \\
\hline FL & SRWMD & -101812013 & - & -101812013 & Alachua & 293813 & 822811 & $5 / 27 / 2010$ & 41 & \\
\hline FL & SRWMD & -101929001 & 293542082253801 & -101929001 & Alachua & 293543 & 822537 & $5 / 19 / 2010$ & 44 & \\
\hline FL & SRWMD & -102006001 & - & -102006001 & Alachua & 293858 & 822040 & $5 / 27 / 2010$ & 46 & \\
\hline FL & SRWMD & -102125001 & - & -102125001 & Alachua & 293555 & 820932 & $5 / 24 / 2010$ & 76 & \\
\hline FL & SRWMD & -111117007 & - & -111117007 & Dixie & 293137 & 831429 & $5 / 4 / 2010$ & 13 & \\
\hline FL & SRWMD & -111327001 & - & -111327001 & Dixie & 292957 & 825939 & $5 / 10 / 2010$ & 13 & \\
\hline FL & SRWMD & -111809001 & - & -111809001 & Alachua & 293228 & 823059 & $5 / 19 / 2010$ & 41 & \\
\hline FL & SRWMD & -111811001 & - & -111811001 & Alachua & 293250 & 822924 & $5 / 26 / 2010$ & 43 & \\
\hline FL & SRWMD & -112136001 & - & -112136001 & Alachua & 292905 & 820947 & $5 / 24 / 2010$ & 51 & \\
\hline FL & SRWMD & -121330002 & - & -121330002 & Dixie & 292448 & 830238 & $5 / 18 / 2010$ & 7 & \\
\hline FL & SRWMD & -131203001 & - & -131203001 & Dixie & 292259 & 830541 & $5 / 5 / 2010$ & 9 & \\
\hline FL & SRWMD & -131433001 & 291806082545601 & -131433001 & Levy & 291809 & 825456 & $5 / 26 / 2010$ & 18 & \\
\hline FL & SRWMD & -131526001 & - & -131526001 & Levy & 291926 & 824630 & $5 / 10 / 2010$ & 21 & \\
\hline FL & SRWMD & -131617001 & - & -131617001 & Levy & 292106 & 824419 & $5 / 10 / 2010$ & 27 & \\
\hline FL & SRWMD & -131732001 & - & -131732001 & Levy & 291828 & 823726 & $5 / 10 / 2010$ & 47 & \\
\hline FL & SRWMD & -131821001 & - & -131821001 & Levy & 292010 & 823058 & $5 / 20 / 2010$ & 44 & \\
\hline FL & SRWMD & -131903001 & 292253082240101 & -131903001 & Marion & 292254 & 822400 & $5 / 25 / 2010$ & 45 & \\
\hline FL & SRWMD & -141305001 & - & -141305001 & Levy & 291748 & 830157 & $5 / 10 / 2010$ & 4 & \\
\hline FL & SRWMD & -141707004 & - & -141707004 & Levy & 291652 & 823920 & $5 / 20 / 2010$ & 24 & \\
\hline FL & SRWMD & -151317001 & 291048083011801 & -151317001 & Levy & 291053 & 830117 & $5 / 26 / 2010$ & 3 & \\
\hline
\end{tabular}


Table 1. Water-level measurements in Florida and parts of Georgia, South Carolina, and Alabama, May-June 2010.—Continued

[NGVD 29, National Geodetic Vertical Datum of 1929. Abbreviations for agency database: ACEPD, Alachua County Environmental Protection Department; FGS, Florida Geological Survey; GSA, Geological Survey of Alabama; NWFWMD, Northwest Florida Water Management District; SFWMD, South Florida Water Management District; SRWMD, Suwannee River Water Management District; USGSALWSC, U.S. Geological Survey Alabama Water Science Center; USGS-FLWSC, U.S. Geological Survey Florida Water Science Center; GAWSC, U.S. Geological Survey Georgia Water Science Center; SCWSC, U.S. Geological Survey South Carolina Water Science Center. Abbreviations for Station names: BLVD., boulevard; CO, county; CR, county road; E, east; HS, high school; IFAS, Institute of Food and Agricultural Services; LK, lake; MW, monitoring well; N, north; NE, northeast; NE, northeast; NF, national forest; NW, northwest; PK, park; R., river; RD., road; SE, southeast; SE, southeast; SR, state road; ST., street; TWR, tower; UFA, Upper Floridan aquifer; —, not available; \#, number; “, inches]

\begin{tabular}{|c|c|c|c|c|c|c|c|c|c|c|}
\hline State & $\begin{array}{c}\text { Agency } \\
\text { database }\end{array}$ & Station name & $\begin{array}{c}\text { USGS site } \\
\text { identification } \\
\text { number }\end{array}$ & $\begin{array}{l}\text { Local agency } \\
\text { unique site } \\
\text { identifier }\end{array}$ & County & $\begin{array}{l}\text { Latitude } \\
\text { (degree } \\
\text { minutes } \\
\text { and } \\
\text { seconds) } \\
\text { [DDMMSS] }\end{array}$ & $\begin{array}{l}\text { Longitude } \\
\text { (degree } \\
\text { minutes } \\
\text { and } \\
\text { seconds) } \\
\text { [DDMMSS] }\end{array}$ & $\begin{array}{c}\text { Date of } \\
\text { measure- } \\
\text { ment }\end{array}$ & $\begin{array}{c}\text { Water } \\
\text { level } \\
\text { altitude } \\
\text { (feet } \\
\text { above } \\
\text { NGVD 29) }\end{array}$ & Remarks \\
\hline FL & SRWMD & $\begin{array}{l}\text { A-0056 GRU-DEERHAVEN } \\
\text { UFA NR GAINESVILLE }\end{array}$ & 294530082232001 & - & Alachua & 294530 & 822320 & $5 / 26 / 2010$ & 40 & \\
\hline FL & SRWMD & BRONSON HIGH SCHOOL & 292744082375201 & - & Levy & 292745 & 823753 & $5 / 10 / 2010$ & 44 & \\
\hline FL & SRWMD & $\begin{array}{l}\text { FANNING SPRINGS STATE } \\
\text { PARK }\end{array}$ & 293513082560601 & - & Levy & 293513 & 825606 & $5 / 18 / 2010$ & 5 & \\
\hline FL & SRWMD & $\begin{array}{l}\text { H.E. MILLS 10" UFA NR } \\
\text { CHIEFLAND }\end{array}$ & 292713082493601 & - & Levy & 292656 & 824956 & $5 / 20 / 2010$ & 19 & \\
\hline FL & SRWMD & Hornsby Springs & 295059082353600 & - & Alachua & 295100 & 823536 & $5 / 18 / 2010$ & 33 & \\
\hline FL & SRWMD & Manatee Springs State Park & 292921082583285 & - & Levy & 292919 & 825831 & $5 / 18 / 2010$ & 4 & \\
\hline FL & SRWMD & NEIL PHILLIPS 4IN & 292507082560201 & - & Levy & 292506 & 825603 & $5 / 26 / 2010$ & 10 & \\
\hline FL & USGS-FLWSC & (City of) Astor Park 8" UFA. & 290900081342002 & - & Lake & 290901 & 813419 & $5 / 24 / 2010$ & 34 & \\
\hline FL & USGS-FLWSC & $\begin{array}{l}\text { (City of) St. Pete 10" UFA \#E- } \\
100 \text { near Citrus Park }\end{array}$ & 280548082355701 & - & Hillsborough & 280549 & 823556 & $5 / 19 / 2010$ & 28 & \\
\hline FL & USGS-FLWSC & $\begin{array}{l}\text { (City of) St. Pete 6" UFA \#E- } \\
102 \text { near Citrus Park }\end{array}$ & 280504082365501 & - & Hillsborough & 280505 & 823654 & $5 / 19 / 2010$ & 23 & \\
\hline FL & USGS-FLWSC & $\begin{array}{l}\text { (City of) Zolfo Springs 16" } \\
\text { UFA-Combo \#1776 }\end{array}$ & 272944081474001 & - & Hardee & 272945 & 814739 & $5 / 20 / 2010$ & 50 & \\
\hline FL & USGS-FLWSC & $\begin{array}{l}\text { 10" UFA Artesian off SR46A } \\
\text { near Sorrento }\end{array}$ & 284929081294901 & - & Lake & 284930 & 812948 & $5 / 17 / 2010$ & 43 & \\
\hline FL & USGS-FLWSC & 672G SR 3 9570; (BR 1843) & 281109080373701 & - & Brevard & 281110 & 803735 & $5 / 25 / 2010$ & 28 & \\
\hline FL & USGS-FLWSC & $\begin{array}{l}\text { 756121—KISS STPK WELL } \\
\text { NR LK KI }\end{array}$ & 275634081211801 & - & Polk & 275635 & 812117 & $5 / 18 / 2010$ & 57 & \\
\hline FL & USGS-FLWSC & $\begin{array}{l}\text { 801127_LK HATCHIN. NR } \\
\text { HAINES C }\end{array}$ & 280153081274101 & - & Polk & 280154 & 812740 & $5 / 19 / 2010$ & 68 & \\
\hline FL & USGS-FLWSC & $\begin{array}{l}\text { 805131-KIMBELL WELL } \\
\text { NR LK MARI }\end{array}$ & 280558081314801 & - & Polk & 280559 & 813147 & $5 / 19 / 2010$ & 71 & \\
\hline FL & USGS-FLWSC & 813149423 26S25E16 312 & 281317081491301 & - & Polk & 281318 & 814912 & $5 / 19 / 2010$ & 126 & \\
\hline
\end{tabular}


Table 1. Water-level measurements in Florida and parts of Georgia, South Carolina, and Alabama, May-June 2010.—Continued

[NGVD 29, National Geodetic Vertical Datum of 1929. Abbreviations for agency database: ACEPD, Alachua County Environmental Protection Department; FGS, Florida Geological Survey; GSA, Geological Survey of Alabama; NWFWMD, Northwest Florida Water Management District; SFWMD, South Florida Water Management District; SRWMD, Suwannee River Water Management District; USGSALWSC, U.S. Geological Survey Alabama Water Science Center; USGS-FLWSC, U.S. Geological Survey Florida Water Science Center; GAWSC, U.S. Geological Survey Georgia Water Science Center; SCWSC, U.S. Geological Survey South Carolina Water Science Center. Abbreviations for Station names: BLVD., boulevard; CO, county; CR, county road; E, east; HS, high school; IFAS, Institute of Food and Agricultural Services; LK, lake; MW, monitoring well; N, north; NE, northeast; NE, northeast; NF, national forest; NW, northwest; PK, park; R., river; RD., road; SE, southeast; SE, southeast; SR, state road; ST., street; TWR, tower; UFA, Upper Floridan aquifer; - - not available; \#, number; “, inches]

\begin{tabular}{|c|c|c|c|c|c|c|c|c|c|c|}
\hline State & $\begin{array}{l}\text { Agency } \\
\text { database }\end{array}$ & Station name & $\begin{array}{c}\text { USGS site } \\
\text { identification } \\
\text { number }\end{array}$ & $\begin{array}{l}\text { Local agency } \\
\text { unique site } \\
\text { identifier }\end{array}$ & County & $\begin{array}{l}\text { Latitude } \\
\text { (degree } \\
\text { minutes } \\
\text { and } \\
\text { seconds) } \\
\text { [DDMMSS] }\end{array}$ & $\begin{array}{l}\text { Longitude } \\
\text { (degree } \\
\text { minutes } \\
\text { and } \\
\text { seconds) } \\
\text { [DDMMSS] }\end{array}$ & $\begin{array}{l}\text { Date of } \\
\text { measure- } \\
\text { ment }\end{array}$ & $\begin{array}{c}\text { Water } \\
\text { level } \\
\text { altitude } \\
\text { (feet } \\
\text { above } \\
\text { NGVD 29) }\end{array}$ & Remarks \\
\hline FL & USGS-FLWSC & 814143232 26S26E04 333 & 281440081431701 & - & Polk & 281441 & 814316 & $5 / 19 / 2010$ & 127 & \\
\hline FL & USGS-FLWSC & $\begin{array}{l}\text { 831-125-04 LK MANN } \\
\text { DRAIN WELL }\end{array}$ & 283144081254201 & - & Orange & 283145 & 812541 & $5 / 18 / 2010$ & 50 & \\
\hline FL & USGS-FLWSC & $\begin{array}{l}\text { A-0038 DRISCOLL UFA AT } \\
\text { LK JEFFORDS }\end{array}$ & 293253082055701 & - & Alachua & 293254 & 820556 & $5 / 24 / 2010$ & 70 & \\
\hline FL & USGS-FLWSC & $\begin{array}{l}\text { A-0053 CELLON 2" UFA NR } \\
\text { LACROSSE }\end{array}$ & 294839082230701 & - & Alachua & 294840 & 822306 & $5 / 25 / 2010$ & 43 & \\
\hline FL & USGS-FLWSC & $\begin{array}{l}\text { A-0068 USGS UFA CE-1A NR } \\
\text { NEWBERRY }\end{array}$ & 293620082362001 & -101722001 & Alachua & 293621 & 823619 & $5 / 25 / 2010$ & 39 & $\begin{array}{l}\text { SRWMD Site iden- } \\
\text { tification number } \\
-101722001\end{array}$ \\
\hline FL & USGS-FLWSC & $\begin{array}{l}\text { A-0071 HAWTHORNE } \\
\text { TOWER UFA }\end{array}$ & 293556082043401 & - & Alachua & 293558 & 820432 & $5 / 24 / 2010$ & 76 & \\
\hline FL & USGS-FLWSC & A-0420 LYBASS AT PHIFER & 293415082112201 & - & Alachua & 293415 & 821122 & $5 / 25 / 2010$ & 67 & \\
\hline FL & USGS-FLWSC & $\begin{array}{l}\text { A-0693 ALACHUA CO FAIR- } \\
\text { GROUNDS UFA }\end{array}$ & 294105082171501 & - & Alachua & 294106 & 821714 & $5 / 25 / 2010$ & 46 & \\
\hline FL & USGS-FLWSC & $\begin{array}{l}\text { A-0725 UFA LK LOCHLOO- } \\
\text { SA CR } 325\end{array}$ & 292838082073701 & - & Alachua & 292839 & 820736 & $5 / 24 / 2010$ & 56 & \\
\hline FL & USGS-FLWSC & $\begin{array}{l}\text { Alexander Springs Creek 4" } \\
\text { UFA, OcalaNF FSR552B } \\
\text { boat ramp }\end{array}$ & 290244081302601 & - & Lake & 290245 & 813025 & $5 / 24 / 2010$ & 16 & \\
\hline FL & USGS-FLWSC & $\begin{array}{l}\text { ALSTON } 1 \text { DEEP (UFA) } \\
\text { WELL }\end{array}$ & 281052082052601 & - & Polk & 281053 & 820525 & $5 / 18 / 2010$ & 96 & \\
\hline FL & USGS-FLWSC & ALSTON TRACK DEEP & 281031082071801 & - & Pasco & 281032 & 820717 & $5 / 18 / 2010$ & 85 & \\
\hline FL & USGS-FLWSC & $\begin{array}{l}\text { ASHTON FORESTRY TOW- } \\
\text { ER 4" UFA, ASHTON }\end{array}$ & 281443081140501 & - & Osceola & 281444 & 811404 & $5 / 17 / 2010$ & 46 & \\
\hline FL & USGS-FLWSC & Austin Smith Rd UFA & 281533082130601 & - & Pasco & 281534 & 821305 & $5 / 17 / 2010$ & 68 & \\
\hline
\end{tabular}


Table 1. Water-level measurements in Florida and parts of Georgia, South Carolina, and Alabama, May-June 2010.—Continued

[NGVD 29, National Geodetic Vertical Datum of 1929. Abbreviations for agency database: ACEPD, Alachua County Environmental Protection Department; FGS, Florida Geological Survey; GSA, Geological Survey of Alabama; NWFWMD, Northwest Florida Water Management District; SFWMD, South Florida Water Management District; SRWMD, Suwannee River Water Management District; USGSALWSC, U.S. Geological Survey Alabama Water Science Center; USGS-FLWSC, U.S. Geological Survey Florida Water Science Center; GAWSC, U.S. Geological Survey Georgia Water Science Center; SCWSC, U.S. Geological Survey South Carolina Water Science Center. Abbreviations for Station names: BLVD., boulevard; CO, county; CR, county road; E, east; HS, high school; IFAS, Institute of Food and Agricultural Services; LK, lake; MW, monitoring well; N, north; NE, northeast; NE, northeast; NF, national forest; NW, northwest; PK, park; R., river; RD., road; SE, southeast; SE, southeast; SR, state road; ST., street; TWR, tower; UFA, Upper Floridan aquifer; —, not available; \#, number; “, inches]

\begin{tabular}{|c|c|c|c|c|c|c|c|c|c|c|}
\hline State & $\begin{array}{l}\text { Agency } \\
\text { database }\end{array}$ & Station name & $\begin{array}{c}\text { USGS site } \\
\text { identification } \\
\text { number }\end{array}$ & $\begin{array}{l}\text { Local agency } \\
\text { unique site } \\
\text { identifier }\end{array}$ & County & $\begin{array}{l}\text { Latitude } \\
\text { (degree } \\
\text { minutes } \\
\text { and } \\
\text { seconds) } \\
\text { [DDMMS] }\end{array}$ & $\begin{array}{l}\text { Longitude } \\
\text { (degree } \\
\text { minutes } \\
\text { and } \\
\text { seconds) } \\
\text { ] [DDMMSS] }\end{array}$ & $\begin{array}{l}\text { Date of } \\
\text { measure- } \\
\text { ment }\end{array}$ & $\begin{array}{c}\text { Water } \\
\text { level } \\
\text { altitude } \\
\text { (feet } \\
\text { above } \\
\text { NGVD 29) }\end{array}$ & Remarks \\
\hline FL & USGS-FLWSC & $\begin{array}{l}\text { AVON PARK PRISON \#1 NR } \\
\text { AIRFIELD AT BOMBING } \\
\text { RANGE }\end{array}$ & 273903081185201 & - & Polk & 273912 & 812107 & $5 / 18 / 2010$ & 70 & \\
\hline FL & USGS-FLWSC & $\begin{array}{l}\text { BA0015 OCEAN POND UFA } \\
\text { (SRM \#-031908001) }\end{array}$ & 301423082261101 & - & Baker & 301424 & 822610 & $5 / 17 / 2010$ & 59 & \\
\hline FL & USGS-FLWSC & BA0019 MANNING 6" UFA & 301022082103301 & - & Baker & 301023 & 821032 & $5 / 17 / 2010$ & 56 & \\
\hline FL & USGS-FLWSC & $\begin{array}{l}\text { BA0054 MACCLENNY FIRE- } \\
\text { TOWER UFA }\end{array}$ & 301618082110901 & - & Baker & 301619 & 821108 & $5 / 17 / 2010$ & 54 & \\
\hline FL & USGS-FLWSC & $\begin{array}{l}\text { BA0057 EDDY FIRETOWER } \\
\text { UFA }\end{array}$ & 303235082203501 & - & Baker & 303236 & 822034 & $5 / 17 / 2010$ & 46 & \\
\hline FL & USGS-FLWSC & $\begin{array}{l}\text { Bardmoor Deep Well near } \\
\text { Pinellas Park, FL }\end{array}$ & 275138082450301 & - & Pinellas & 275142 & 824502 & $5 / 17 / 2010$ & 8 & \\
\hline FL & USGS-FLWSC & $\begin{array}{l}\text { BARNHART WELL CE25 AT } \\
\text { NOBLETON }\end{array}$ & 283840082154801 & - & Hernando & 283841 & 821547 & $5 / 18 / 2010$ & 39 & \\
\hline FL & USGS-FLWSC & $\begin{array}{l}\text { Barthle Ranch 6" UFA near } \\
\text { Darby }\end{array}$ & 282534082222802 & - & Pasco & 282535 & 822227 & $5 / 17 / 2010$ & 39 & \\
\hline FL & USGS-FLWSC & $\begin{array}{l}\text { Bartow WWTP-LWIP SWNN } \\
\text { Well at Bartow, FL }\end{array}$ & 275336081484401 & - & Polk & 275336 & 814844 & $5 / 20 / 2010$ & 75 & \\
\hline FL & USGS-FLWSC & $\begin{array}{l}\text { BAY LAKE DP WELL NR } \\
\text { BAY LAKE }\end{array}$ & 282528081340901 & - & Orange & 282529 & 813408 & $5 / 18 / 2010$ & 85 & \\
\hline FL & USGS-FLWSC & $\begin{array}{l}\text { Beneke FLDN Well near } \\
\text { Odessa, FL }\end{array}$ & 281125082344401 & - & Pasco & 281125 & 823444 & $5 / 18 / 2010$ & 42 & \\
\hline FL & USGS-FLWSC & Berger 6" UFA near Citrus Park & 280659082294302 & - & Hillsborough & 280700 & 822942 & $5 / 19 / 2010$ & 48 & \\
\hline FL & USGS-FLWSC & Bexley 8" UFA \#2 near Drexel & 281448082301801 & - & Pasco & 281449 & 823017 & $5 / 17 / 2010$ & 61 & \\
\hline FL & USGS-FLWSC & $\begin{array}{l}\text { BLACK AND TURNER FLRD } \\
\text { WELL AT LITHIA }\end{array}$ & 275117082090001 & - & Hillsborough & 275118 & 820859 & $5 / 18 / 2010$ & 25 & \\
\hline
\end{tabular}


Table 1. Water-level measurements in Florida and parts of Georgia, South Carolina, and Alabama, May-June 2010.—Continued

[NGVD 29, National Geodetic Vertical Datum of 1929. Abbreviations for agency database: ACEPD, Alachua County Environmental Protection Department; FGS, Florida Geological Survey; GSA, Geological Survey of Alabama; NWFWMD, Northwest Florida Water Management District; SFWMD, South Florida Water Management District; SRWMD, Suwannee River Water Management District; USGSALWSC, U.S. Geological Survey Alabama Water Science Center; USGS-FLWSC, U.S. Geological Survey Florida Water Science Center; GAWSC, U.S. Geological Survey Georgia Water Science Center; SCWSC, U.S. Geological Survey South Carolina Water Science Center. Abbreviations for Station names: BLVD., boulevard; CO, county; CR, county road; E, east; HS, high school; IFAS, Institute of Food and Agricultural Services; LK, lake; MW, monitoring well; N, north; NE, northeast; NE, northeast; NF, national forest; NW, northwest; PK, park; R., river; RD., road; SE, southeast; SE, southeast; SR, state road; ST., street; TWR, tower; UFA, Upper Floridan aquifer; - - not available; \#, number; “, inches]

\begin{tabular}{|c|c|c|c|c|c|c|c|c|c|c|}
\hline State & $\begin{array}{c}\text { Agency } \\
\text { database }\end{array}$ & Station name & $\begin{array}{c}\text { USGS site } \\
\text { identification } \\
\text { number }\end{array}$ & $\begin{array}{l}\text { Local agency } \\
\text { unique site } \\
\text { identifier }\end{array}$ & County & $\begin{array}{l}\text { Latitude } \\
\text { (degree } \\
\text { minutes } \\
\text { and } \\
\text { seconds) } \\
\text { [DDMMSS }\end{array}$ & $\begin{array}{l}\text { Longitude } \\
\text { (degree } \\
\text { minutes } \\
\text { and } \\
\text { seconds) } \\
\text { ] [DDMMSS] }\end{array}$ & $\begin{array}{c}\text { Date of } \\
\text { measure- } \\
\text { ment }\end{array}$ & $\begin{array}{c}\text { Water } \\
\text { level } \\
\text { altitude } \\
\text { (feet } \\
\text { above } \\
\text { NGVD 29) }\end{array}$ & Remarks \\
\hline FL & USGS-FLWSC & $\begin{array}{l}\text { BLACKETT REPLACEMENT } \\
\text { WELL NEAR LAKE NEFF }\end{array}$ & 282839082190802 & - & Hernando & 282840 & 821907 & $5 / 18 / 2010$ & 73 & \\
\hline FL & USGS-FLWSC & $\begin{array}{l}\text { BLACWATER CREEK ELAPP } \\
\text { UFA WELL }\end{array}$ & 280740082105201 & - & Hillsborough & 280741 & 821051 & $5 / 18 / 2010$ & 71 & \\
\hline FL & USGS-FLWSC & $\begin{array}{l}\text { Bonnet Lake 6" UFA near } \\
\text { Sebring }\end{array}$ & 273252081264101 & - & Highlands & 273253 & 812640 & $5 / 19 / 2010$ & 74 & \\
\hline FL & USGS-FLWSC & $\begin{array}{l}\text { BOYETTE REPLACEMENT } \\
\text { WELL }\end{array}$ & 282840082035501 & - & Hernando & 282841 & 820355 & $5 / 18 / 2010$ & 86 & \\
\hline FL & USGS-FLWSC & $\begin{array}{l}\text { BR0001 2" UFA, Champion } \\
\text { Rd, Titusville }\end{array}$ & 283627080512001 & - & Brevard & 283628 & 805119 & $5 / 24 / 2010$ & 16 & \\
\hline FL & USGS-FLWSC & $\begin{array}{l}\text { BR0107 CHAPEL BY THE } \\
\text { SEA }\end{array}$ & 275425080283101 & - & Brevard & 275426 & 802830 & $5 / 25 / 2010$ & 34 & \\
\hline FL & USGS-FLWSC & BR0208 JETTY PARK & 282423080353601 & - & Brevard & 282424 & 803535 & $5 / 25 / 2010$ & 21 & \\
\hline FL & USGS-FLWSC & $\begin{array}{l}\text { BR0299 BOGGS } 6215 \text { SR3 S } \\
\text { TROPIC TRAIL }\end{array}$ & 281447080392601 & - & Brevard & 281448 & 803925 & $5 / 25 / 2010$ & 27 & \\
\hline FL & USGS-FLWSC & $\begin{array}{l}\text { BR0341 BREVARD HARD- } \\
\text { WOODS }\end{array}$ & 280008080342601 & - & Brevard & 280009 & 803425 & $5 / 25 / 2010$ & 32 & \\
\hline FL & USGS-FLWSC & $\begin{array}{l}\text { BR0424 VISCONTI WATER- } \\
\text { MAN DRIVE }\end{array}$ & 275948080393501 & - & Brevard & 275949 & 803934 & $5 / 24 / 2010$ & 38 & \\
\hline FL & USGS-FLWSC & $\begin{array}{l}\text { BR0585 ASTRONAUT HS } \\
\text { UFA }\end{array}$ & 283732080510001 & - & Brevard & 283733 & 805059 & $5 / 24 / 2010$ & 12 & \\
\hline FL & USGS-FLWSC & $\begin{array}{l}\text { BR0586 TICO AIRPORT NR } \\
\text { TITUSVILLE }\end{array}$ & 282945080473901 & - & Brevard & 282946 & 804738 & $5 / 24 / 2010$ & 17 & \\
\hline FL & USGS-FLWSC & $\begin{array}{l}\text { BR0608 NASA 4" UFA near } \\
\text { Gate } 2\end{array}$ & 282921080404701 & - & Brevard & 282922 & 804046 & $5 / 25 / 2010$ & 14 & \\
\hline FL & USGS-FLWSC & $\begin{array}{l}\text { BR0625 SEBASTIAN ST PK } \\
\quad 450\end{array}$ & 275210080272202 & - & Brevard & 275211 & 802721 & $5 / 25 / 2010$ & 34 & \\
\hline
\end{tabular}


Table 1. Water-level measurements in Florida and parts of Georgia, South Carolina, and Alabama, May-June 2010.—Continued

[NGVD 29, National Geodetic Vertical Datum of 1929. Abbreviations for agency database: ACEPD, Alachua County Environmental Protection Department; FGS, Florida Geological Survey; GSA, Geological Survey of Alabama; NWFWMD, Northwest Florida Water Management District; SFWMD, South Florida Water Management District; SRWMD, Suwannee River Water Management District; USGSALWSC, U.S. Geological Survey Alabama Water Science Center; USGS-FLWSC, U.S. Geological Survey Florida Water Science Center; GAWSC, U.S. Geological Survey Georgia Water Science Center; SCWSC, U.S. Geological Survey South Carolina Water Science Center. Abbreviations for Station names: BLVD., boulevard; CO, county; CR, county road; E, east; HS, high school; IFAS, Institute of Food and Agricultural Services; LK, lake; MW, monitoring well; N, north; NE, northeast; NE, northeast; NF, national forest; NW, northwest; PK, park; R., river; RD., road; SE, southeast; SE, southeast; SR, state road; ST., street; TWR, tower; UFA, Upper Floridan aquifer; —, not available; \#, number; “, inches]

\begin{tabular}{|c|c|c|c|c|c|c|c|c|c|c|}
\hline State & $\begin{array}{l}\text { Agency } \\
\text { database }\end{array}$ & Station name & $\begin{array}{c}\text { USGS site } \\
\text { identification } \\
\text { number }\end{array}$ & $\begin{array}{l}\text { Local agency } \\
\text { unique site } \\
\text { identifier }\end{array}$ & County & $\begin{array}{l}\text { Latitude } \\
\text { (degree } \\
\text { minutes } \\
\text { and } \\
\text { seconds) } \\
\text { [DDMMSS] }\end{array}$ & $\begin{array}{l}\text { Longitude } \\
\text { (degree } \\
\text { minutes } \\
\text { and } \\
\text { seconds) } \\
\text { [DDMMSS] }\end{array}$ & $\begin{array}{l}\text { Date of } \\
\text { measure- } \\
\text { ment }\end{array}$ & $\begin{array}{c}\text { Water } \\
\text { level } \\
\text { altitude } \\
\text { (feet } \\
\text { above } \\
\text { NGVD 29) }\end{array}$ & Remarks \\
\hline FL & USGS-FLWSC & $\begin{array}{l}\text { BR0645 PLATT WELL NR } \\
\text { MALABAR }\end{array}$ & 275955080434601 & - & Brevard & 275956 & 804345 & $5 / 24 / 2010$ & 40 & \\
\hline FL & USGS-FLWSC & $\begin{array}{l}\text { BR1050 MERRITT ISLE } \\
\text { WILDLIFE }\end{array}$ & 283835080424501 & - & Brevard & 283837 & 804243 & $5 / 24 / 2010$ & 11 & \\
\hline FL & USGS-FLWSC & $\begin{array}{l}\text { BR1526 SEMINOLE RANCH } \\
\text { 6" UFA }\end{array}$ & 283644080574903 & - & Brevard & 283645 & 805748 & $5 / 24 / 2010$ & 19 & \\
\hline FL & USGS-FLWSC & BR1557 COCOA HS & 282301080460601 & - & Brevard & 282302 & 804605 & $5 / 24 / 2010$ & 24 & \\
\hline FL & USGS-FLWSC & BR1558 KENNEDY HS & 281937080442001 & - & Brevard & 281938 & 804419 & $5 / 24 / 2010$ & 27 & \\
\hline FL & USGS-FLWSC & $\begin{array}{l}\text { BR1559 FLEMING GRANT } \\
\text { (BR1550) }\end{array}$ & 275003080330201 & - & Brevard & 275004 & 803301 & $5 / 25 / 2010$ & 39 & \\
\hline FL & USGS-FLWSC & $\begin{array}{l}\text { BR1746 } 192 \text { CO LINE DEER } \\
\text { PARK SE (Brevard Landfill } \\
\text { Site) }\end{array}$ & 280532080514501 & - & Brevard & 280533 & 805144 & $5 / 24 / 2010$ & 41 & \\
\hline FL & USGS-FLWSC & $\begin{array}{l}\text { BR1748 KSC GROVE 6" UFA, } \\
\text { RANGE RD }\end{array}$ & 283028080403501 & - & Brevard & 283029 & 804034 & $5 / 25 / 2010$ & 13 & \\
\hline FL & USGS-FLWSC & $\begin{array}{l}\text { BRENTWOOD WELL NR } \\
\text { SUMTERVILLE }\end{array}$ & 284435082011701 & - & Sumter & 284436 & 820116 & $5 / 18 / 2010$ & 54 & \\
\hline FL & USGS-FLWSC & $\begin{array}{l}\text { BULLOCK-HUBER 4" UFA } \\
\text { NR WILLISTON }\end{array}$ & 291910082341101 & - & Levy & 291904 & 823411 & $5 / 26 / 2010$ & 43 & \\
\hline FL & USGS-FLWSC & $\begin{array}{l}\text { BURNT HOUSE WELL } \\
\text { LOWE }\end{array}$ & 284703082001701 & - & Sumter & 284704 & 820016 & $5 / 18 / 2010$ & 55 & \\
\hline FL & USGS-FLWSC & Busby 12" UFA near Duette & 273605082071101 & - & Manatee & 273606 & 820710 & $5 / 18 / 2010$ & 28 & \\
\hline FL & USGS-FLWSC & $\begin{array}{l}\text { BYRD TRAILER WELL NR } \\
\text { ORANGE HOME }\end{array}$ & 284955081595801 & - & Sumter & 284956 & 815957 & $5 / 19 / 2010$ & 65 & \\
\hline FL & USGS-FLWSC & $\begin{array}{l}\text { C-0009 COUNTYLINE 4" } \\
\text { UFA NR MELROSE }\end{array}$ & 294307082020903 & -092307001 & Clay & 294314 & 820245 & $5 / 18 / 2010$ & 83 & $\begin{array}{l}\text { SRWMD Site iden- } \\
\text { tification number } \\
-092307001\end{array}$ \\
\hline
\end{tabular}


Table 1. Water-level measurements in Florida and parts of Georgia, South Carolina, and Alabama, May-June 2010.-Continued

[NGVD 29, National Geodetic Vertical Datum of 1929. Abbreviations for agency database: ACEPD, Alachua County Environmental Protection Department; FGS, Florida Geological Survey; GSA, Geological Survey of Alabama; NWFWMD, Northwest Florida Water Management District; SFWMD, South Florida Water Management District; SRWMD, Suwannee River Water Management District; USGSALWSC, U.S. Geological Survey Alabama Water Science Center; USGS-FLWSC, U.S. Geological Survey Florida Water Science Center; GAWSC, U.S. Geological Survey Georgia Water Science Center; SCWSC, U.S. Geological Survey South Carolina Water Science Center. Abbreviations for Station names: BLVD., boulevard; CO, county; CR, county road; E, east; HS, high school; IFAS, Institute of Food and Agricultural Services; LK, lake; MW, monitoring well; N, north; NE, northeast; NE, northeast; NF, national forest; NW, northwest; PK, park; R., river; RD., road; SE, southeast; SE, southeast; SR, state road; ST., street; TWR, tower; UFA, Upper Floridan aquifer; - - not available; \#, number; “, inches]

\begin{tabular}{|c|c|c|c|c|c|c|c|c|c|c|}
\hline State & $\begin{array}{l}\text { Agency } \\
\text { database }\end{array}$ & Station name & $\begin{array}{c}\text { USGS site } \\
\text { identification } \\
\text { number }\end{array}$ & $\begin{array}{l}\text { Local agency } \\
\text { unique site } \\
\text { identifier }\end{array}$ & County & $\begin{array}{l}\text { Latitude } \\
\text { (degree } \\
\text { minutes } \\
\text { and } \\
\text { seconds) } \\
\text { [DDMMSS] }\end{array}$ & $\begin{array}{l}\text { Longitude } \\
\text { (degree } \\
\text { minutes } \\
\text { and } \\
\text { seconds) } \\
\text { ] [DDMMSS] }\end{array}$ & $\begin{array}{c}\text { Date of } \\
\text { measure- } \\
\text { ment }\end{array}$ & $\begin{array}{c}\text { Water } \\
\text { level } \\
\text { altitude } \\
\text { (feet } \\
\text { above } \\
\text { NGVD 29) }\end{array}$ & Remarks \\
\hline FL & USGS-FLWSC & C-0120 KEYSTONE HTS UFA & 294807082020903 & - & Clay & 294808 & 820208 & $5 / 18 / 2010$ & 81 & \\
\hline FL & USGS-FLWSC & $\begin{array}{l}\text { C-0123 SUNGARDEN TWR } \\
\text { OCALA 4" UFA }\end{array}$ & 295016081433501 & - & Clay & 295017 & 814333 & $5 / 18 / 2010$ & 70 & \\
\hline FL & USGS-FLWSC & $\begin{array}{l}\text { C-0128 PENNEY FARMS } \\
\text { TOWER 4" UFA }\end{array}$ & 295851081555301 & - & Clay & 295852 & 815552 & $5 / 18 / 2010$ & 70 & \\
\hline FL & USGS-FLWSC & $\begin{array}{l}\text { C-0442 CHESTER MOODY } \\
\text { 6" UFA }\end{array}$ & 294728082010901 & - & Clay & 294729 & 820108 & $5 / 18 / 2010$ & 80 & \\
\hline FL & USGS-FLWSC & C-0453 GOLD HEAD 6" UFA & 294911081572601 & - & Clay & 294913 & 815723 & $5 / 18 / 2010$ & 78 & \\
\hline FL & USGS-FLWSC & $\begin{array}{l}\text { C-0495 THUNDERBOLT } \\
\text { ELEMENTARY }\end{array}$ & 300502081432301 & - & Clay & 300502 & 814323 & $5 / 19 / 2010$ & 30 & \\
\hline FL & USGS-FLWSC & $\begin{array}{l}\text { C-0579 JP HALL 6" UFA, } \\
\text { BAYARD POINT }\end{array}$ & 295733081365505 & - & Clay & 295734 & 813655 & $5 / 18 / 2010$ & 26 & \\
\hline FL & USGS-FLWSC & $\begin{array}{l}\text { C-0583 YELLOW WATER } \\
\text { CREEK 6" UFA }\end{array}$ & 300926081561603 & - & Clay & 300927 & 815615 & $5 / 17 / 2010$ & 54 & \\
\hline FL & USGS-FLWSC & $\begin{array}{l}\text { C-0592 TYNES ELEMEN- } \\
\text { TARY }\end{array}$ & 300705081505401 & - & Clay & 300705 & 815054 & $5 / 20 / 2010$ & 48 & \\
\hline FL & USGS-FLWSC & $\begin{array}{l}\text { C-0599 CANVASBACK RD } \\
\text { NR MIDDLEBURG }\end{array}$ & 300540081583801 & - & Clay & 300540 & 815838 & $5 / 17 / 2010$ & 59 & \\
\hline FL & USGS-FLWSC & C-0607 SR 16 BLACK CREEK & 295841081514701 & - & Clay & 295841 & 815147 & $5 / 18 / 2010$ & 71 & \\
\hline FL & USGS-FLWSC & $\begin{array}{l}\text { C-1011 SR21/CR315C 6" UFA, } \\
\text { CAMP BLANDING }\end{array}$ & 295238081553701 & - & Clay & 295239 & 815536 & $5 / 19 / 2010$ & 75 & \\
\hline FL & USGS-FLWSC & $\begin{array}{l}\text { C-1014 CAMP BLANDING @ } \\
\text { SR } 16\end{array}$ & 295859082003903 & - & Clay & 295860 & 820038 & $5 / 19 / 2010$ & 65 & \\
\hline FL & USGS-FLWSC & $\begin{array}{l}\text { C-1017 CAMP BLANDING } \\
\text { NORTH PERIMETER 6" } \\
\text { UFA }\end{array}$ & 300318082015401 & - & Clay & 300319 & 820153 & $5 / 19 / 2010$ & 60 & \\
\hline
\end{tabular}


Table 1. Water-level measurements in Florida and parts of Georgia, South Carolina, and Alabama, May-June 2010.—Continued

[NGVD 29, National Geodetic Vertical Datum of 1929. Abbreviations for agency database: ACEPD, Alachua County Environmental Protection Department; FGS, Florida Geological Survey; GSA, Geological Survey of Alabama; NWFWMD, Northwest Florida Water Management District; SFWMD, South Florida Water Management District; SRWMD, Suwannee River Water Management District; USGSALWSC, U.S. Geological Survey Alabama Water Science Center; USGS-FLWSC, U.S. Geological Survey Florida Water Science Center; GAWSC, U.S. Geological Survey Georgia Water Science Center; SCWSC, U.S. Geological Survey South Carolina Water Science Center. Abbreviations for Station names: BLVD., boulevard; CO, county; CR, county road; E, east; HS, high school; IFAS, Institute of Food and Agricultural Services; LK, lake; MW, monitoring well; N, north; NE, northeast; NE, northeast; NF, national forest; NW, northwest; PK, park; R., river; RD., road; SE, southeast; SE, southeast; SR, state road; ST., street; TWR, tower; UFA, Upper Floridan aquifer; —, not available; \#, number; “, inches]

\begin{tabular}{|c|c|c|c|c|c|c|c|c|c|c|}
\hline State & $\begin{array}{c}\text { Agency } \\
\text { database }\end{array}$ & Station name & $\begin{array}{c}\text { USGS site } \\
\text { identification } \\
\text { number }\end{array}$ & $\begin{array}{l}\text { Local agency } \\
\text { unique site } \\
\text { identifier }\end{array}$ & County & $\begin{array}{c}\text { Latitude } \\
\text { (degree } \\
\text { minutes } \\
\text { and } \\
\text { seconds) } \\
\text { [DDMMSS] }\end{array}$ & $\begin{array}{l}\text { Longitude } \\
\text { (degree } \\
\text { minutes } \\
\text { and } \\
\text { seconds) } \\
\text { ] [DDMMSS] }\end{array}$ & $\begin{array}{l}\text { Date of } \\
\text { measure- } \\
\text { ment }\end{array}$ & $\begin{array}{c}\text { Water } \\
\text { level } \\
\text { altitude } \\
\text { (feet } \\
\text { above } \\
\text { NGVD 29) }\end{array}$ & Remarks \\
\hline FL & USGS-FLWSC & $\begin{array}{l}\text { C-1026 BAYARD WELL NR } \\
\text { WALKILL }\end{array}$ & 295222081393501 & - & Clay & 295223 & 813934 & $5 / 18 / 2010$ & 44 & \\
\hline FL & USGS-FLWSC & $\begin{array}{l}\text { C-1056 MONITOR WELL AT } \\
\text { WALKILL }\end{array}$ & 295625081410901 & - & Clay & 295626 & 814108 & $5 / 18 / 2010$ & 47 & \\
\hline FL & USGS-FLWSC & $\begin{array}{l}\text { C-1063 LAKE ASBURY NR } \\
\text { MIDDLEBURG }\end{array}$ & 300338081500301 & - & Clay & 300338 & 815002 & $5 / 17 / 2010$ & 56 & \\
\hline FL & USGS-FLWSC & $\begin{array}{l}\text { Camp Dorothy Htrn Well Nr } \\
\text { Riverview }\end{array}$ & 274947082145401 & - & Hillsborough & 274948 & 821453 & $5 / 18 / 2010$ & 11 & \\
\hline FL & USGS-FLWSC & $\begin{array}{l}\text { Camp McQuarrie (4-H Club } \\
\text { Found.) 6" UFA off SR40, } \\
\text { OcalaNF }\end{array}$ & 290910081360001 & - & Lake & 290912 & 813601 & $5 / 24 / 2010$ & 46 & \\
\hline FL & USGS-FLWSC & $\begin{array}{l}\text { Campground UFA near River- } \\
\text { view }\end{array}$ & 275210082171001 & - & Hillsborough & 275211 & 821709 & $5 / 19 / 2010$ & 10 & \\
\hline FL & USGS-FLWSC & $\begin{array}{l}\text { CANOE CREEK RD WELL } \\
\text { NR ST CLOUD FL }\end{array}$ & 280750081155701 & - & Osceola & 280751 & 811609 & $5 / 17 / 2010$ & 50 & \\
\hline FL & USGS-FLWSC & Catchings 4" UFA near Drexel & 281631082261601 & - & Pasco & 281632 & 822615 & $5 / 21 / 2010$ & 67 & \\
\hline FL & USGS-FLWSC & CE 12 & 290739082245701 & - & Marion & 290740 & 822456 & $5 / 24 / 2010$ & 35 & \\
\hline FL & USGS-FLWSC & CE 24 DRAKE RANCH & 285933082192501 & - & Marion & 285934 & 821924 & $5 / 24 / 2010$ & 38 & \\
\hline FL & USGS-FLWSC & $\begin{array}{l}\text { CE } 6 \text { SR } 488 \text { E OF RED } \\
\text { LEVEL }\end{array}$ & 285951082350901 & - & Citrus & 285952 & 823508 & $5 / 20 / 2010$ & 19 & \\
\hline FL & USGS-FLWSC & CE 75 & 290227082250801 & - & Marion & 290228 & 822507 & $5 / 24 / 2010$ & 55 & \\
\hline FL & USGS-FLWSC & CE 88 US 19-98,N OF CFBC & 290107082400501 & - & Citrus & 290108 & 824004 & $5 / 20 / 2010$ & 4 & \\
\hline FL & USGS-FLWSC & $\begin{array}{l}\text { CE-5 6" UFA Artesian near } \\
\text { Inglis }\end{array}$ & 290112082371101 & - & Levy & 290113 & 823710 & $5 / 19 / 2010$ & 6 & \\
\hline FL & USGS-FLWSC & Central Baptist Youth Camp 4" & 290052081271201 & - & Lake & 290053 & 812711 & $5 / 26 / 2010$ & 44 & \\
\hline
\end{tabular}


Table 1. Water-level measurements in Florida and parts of Georgia, South Carolina, and Alabama, May-June 2010.-Continued

[NGVD 29, National Geodetic Vertical Datum of 1929. Abbreviations for agency database: ACEPD, Alachua County Environmental Protection Department; FGS, Florida Geological Survey; GSA, Geological Survey of Alabama; NWFWMD, Northwest Florida Water Management District; SFWMD, South Florida Water Management District; SRWMD, Suwannee River Water Management District; USGSALWSC, U.S. Geological Survey Alabama Water Science Center; USGS-FLWSC, U.S. Geological Survey Florida Water Science Center; GAWSC, U.S. Geological Survey Georgia Water Science Center; SCWSC, U.S. Geological Survey South Carolina Water Science Center. Abbreviations for Station names: BLVD., boulevard; CO, county; CR, county road; E, east; HS, high school; IFAS, Institute of Food and Agricultural Services; LK, lake; MW, monitoring well; N, north; NE, northeast; NE, northeast; NF, national forest; NW, northwest; PK, park; R., river; RD., road; SE, southeast; SE, southeast; SR, state road; ST., street; TWR, tower; UFA, Upper Floridan aquifer; - - not available; \#, number; “, inches]

\begin{tabular}{|c|c|c|c|c|c|c|c|c|c|c|}
\hline State & $\begin{array}{l}\text { Agency } \\
\text { database }\end{array}$ & Station name & $\begin{array}{c}\text { USGS site } \\
\text { identification } \\
\text { number }\end{array}$ & $\begin{array}{l}\text { Local agency } \\
\text { unique site } \\
\text { identifier }\end{array}$ & County & $\begin{array}{l}\text { Latitude } \\
\text { (degree } \\
\text { minutes } \\
\text { and } \\
\text { seconds) } \\
\text { [DDMMSS }\end{array}$ & $\begin{array}{l}\text { Longitude } \\
\text { (degree } \\
\text { minutes } \\
\text { and } \\
\text { seconds) } \\
\text { [DDMMSS] }\end{array}$ & $\begin{array}{c}\text { Date of } \\
\text { measure- } \\
\text { ment }\end{array}$ & $\begin{array}{c}\text { Water } \\
\text { level } \\
\text { altitude } \\
\text { (feet } \\
\text { above } \\
\text { NGVD 29) }\end{array}$ & Remarks \\
\hline FL & USGS-FLWSC & $\begin{array}{l}\text { CF Ind D 8" UFA \#LF-1 near } \\
\text { Ft. Green }\end{array}$ & 273424081582501 & - & Hardee & 273425 & 815824 & $5 / 17 / 2010$ & 48 & \\
\hline FL & USGS-FLWSC & $\begin{array}{l}\text { CF Ind D 8" UFA \#LF-6 near } \\
\text { Ft. Green }\end{array}$ & 273426081513401 & - & Hardee & 273427 & 815133 & $5 / 17 / 2010$ & 61 & \\
\hline FL & USGS-FLWSC & Chancey Rd UFA & 281322082084501 & - & Pasco & 281323 & 820844 & $5 / 17 / 2010$ & 72 & \\
\hline FL & USGS-FLWSC & $\begin{array}{l}\text { Channel G BM 4" UFA, Town } \\
\text { N' Country }\end{array}$ & 280038082340201 & - & Hillsborough & 280039 & 823401 & $5 / 20 / 2010$ & 7 & \\
\hline FL & USGS-FLWSC & $\begin{array}{c}\text { Chassahowitzka 4" UFA \#1 } \\
\text { near Chassahowitzka }\end{array}$ & 284317082330601 & - & Citrus & 284318 & 823305 & $5 / 17 / 2010$ & 6 & \\
\hline FL & USGS-FLWSC & $\begin{array}{l}\text { CI-1 10" UFA near Babson } \\
\text { Park }\end{array}$ & 275023081321601 & - & Polk & 275024 & 813214 & $5 / 18 / 2010$ & 94 & \\
\hline FL & USGS-FLWSC & $\begin{array}{l}\text { CITRUS } 11 \text { USGS-WEST } \\
\text { WITH RIVER }\end{array}$ & 285105082135802 & - & Citrus & 285102 & 821357 & $5 / 21 / 2010$ & 38 & \\
\hline FL & USGS-FLWSC & $\begin{array}{l}\text { CITRUS SPRINGS GOLF } \\
\text { COURSE WELL }\end{array}$ & 285930082283702 & - & Citrus & 285931 & 822836 & $5 / 20 / 2010$ & 7 & \\
\hline FL & USGS-FLWSC & $\begin{array}{l}\text { Clear Springs Upper Floridan } \\
\text { Well near Bartow, FL }\end{array}$ & 275156081481901 & - & Polk & 275158 & 814819 & $5 / 17 / 2010$ & 77 & \\
\hline FL & USGS-FLWSC & $\begin{array}{l}\text { CLEARWATER DUNEDIN } 27 \\
\text { DEEP }\end{array}$ & 275753082433701 & - & Pinellas & 275754 & 824336 & $5 / 18 / 2010$ & 8 & \\
\hline FL & USGS-FLWSC & $\begin{array}{l}\text { Cle-Dun 10" UFA \#31 near } \\
\text { Clearwater }\end{array}$ & 275753082435301 & - & Pinellas & 275754 & 824352 & $5 / 18 / 2010$ & 8 & \\
\hline FL & USGS-FLWSC & Cmp.Henderson & 305940086580601 & - & Santa Rosa & 305940 & 865805 & $5 / 20 / 2010$ & 108 & \\
\hline FL & USGS-FLWSC & $\begin{array}{l}\text { Coastal Pasco 4" UFA \#04, Port } \\
\text { Richey }\end{array}$ & 281642082440201 & - & Pasco & 281643 & 824401 & $5 / 19 / 2010$ & 0 & \\
\hline FL & USGS-FLWSC & $\begin{array}{l}\text { Coastal Pasco 4" UFA \#13 near } \\
\text { New Port Richey }\end{array}$ & 281023082450701 & - & Pasco & 281024 & 824506 & $5 / 19 / 2010$ & 7 & \\
\hline
\end{tabular}


Table 1. Water-level measurements in Florida and parts of Georgia, South Carolina, and Alabama, May-June 2010.—Continued

[NGVD 29, National Geodetic Vertical Datum of 1929. Abbreviations for agency database: ACEPD, Alachua County Environmental Protection Department; FGS, Florida Geological Survey; GSA, Geological Survey of Alabama; NWFWMD, Northwest Florida Water Management District; SFWMD, South Florida Water Management District; SRWMD, Suwannee River Water Management District; USGSALWSC, U.S. Geological Survey Alabama Water Science Center; USGS-FLWSC, U.S. Geological Survey Florida Water Science Center; GAWSC, U.S. Geological Survey Georgia Water Science Center; SCWSC, U.S. Geological Survey South Carolina Water Science Center. Abbreviations for Station names: BLVD., boulevard; CO, county; CR, county road; E, east; HS, high school; IFAS, Institute of Food and Agricultural Services; LK, lake; MW, monitoring well; N, north; NE, northeast; NE, northeast; NF, national forest; NW, northwest; PK, park; R., river; RD., road; SE, southeast; SE, southeast; SR, state road; ST., street; TWR, tower; UFA, Upper Floridan aquifer; —, not available; \#, number; “, inches]

\begin{tabular}{|c|c|c|c|c|c|c|c|c|c|c|}
\hline State & $\begin{array}{l}\text { Agency } \\
\text { database }\end{array}$ & Station name & $\begin{array}{c}\text { USGS site } \\
\text { identification } \\
\text { number }\end{array}$ & $\begin{array}{l}\text { Local agency } \\
\text { unique site } \\
\text { identifier }\end{array}$ & County & $\begin{array}{c}\text { Latitude } \\
\text { (degree } \\
\text { minutes } \\
\text { and } \\
\text { seconds) } \\
\text { [DDMMSS] }\end{array}$ & $\begin{array}{l}\text { Longitude } \\
\text { (degree } \\
\text { minutes } \\
\text { and } \\
\text { seconds) } \\
\text { [DDMMSS] }\end{array}$ & $\begin{array}{c}\text { Date of } \\
\text { measure- } \\
\text { ment }\end{array}$ & $\begin{array}{c}\text { Water } \\
\text { level } \\
\text { altitude } \\
\text { (feet } \\
\text { above } \\
\text { NGVD 29) }\end{array}$ & Remarks \\
\hline FL & USGS-FLWSC & $\begin{array}{l}\text { Coastal Pasco 4" UFA \#2, } \\
\text { Hudson }\end{array}$ & 282229082405801 & - & Pasco & 282230 & 824057 & $5 / 19 / 2010$ & 1 & \\
\hline FL & USGS-FLWSC & COCOA A 10" UFA & 282341081040101 & - & Orange & 282342 & 810400 & $5 / 19 / 2010$ & 36 & \\
\hline FL & USGS-FLWSC & COCOA P & 282623081153801 & - & Orange & 282624 & 811537 & $5 / 17 / 2010$ & 44 & \\
\hline FL & USGS-FLWSC & Coley 6" UFA, Frostproof & 274440081314801 & - & Polk & 274441 & 813147 & $5 / 18 / 2010$ & 78 & \\
\hline FL & USGS-FLWSC & County line Trade Center & 282557082364301 & - & Hernando & 282556 & 823642 & $5 / 19 / 2010$ & 14 & \\
\hline FL & USGS-FLWSC & Cove Cay 6" UFA near Largo & 275604082431701 & - & Pinellas & 275605 & 824316 & $5 / 18 / 2010$ & 5 & \\
\hline FL & USGS-FLWSC & Cresent Dr 20" UFA, Lakeland & 280520081575201 & - & Polk & 280521 & 815751 & $5 / 21 / 2010$ & 96 & \\
\hline FL & USGS-FLWSC & $\begin{array}{l}\text { CROOM RR SIDING WELL } \\
\text { NR CROOM }\end{array}$ & 283510082133701 & - & Hernando & 283511 & 821336 & $5 / 18 / 2010$ & 41 & \\
\hline FL & USGS-FLWSC & Crossbar WF A-1 6" UFA & 282148082281801 & - & Pasco & 282149 & 822817 & $5 / 17 / 2010$ & 57 & \\
\hline FL & USGS-FLWSC & $\begin{array}{l}\text { Crystal River 6" UFA, Crystal } \\
\text { River }\end{array}$ & 285421082361602 & - & Citrus & 285422 & 823615 & $5 / 18 / 2010$ & 1 & \\
\hline FL & USGS-FLWSC & CUMMER OFFICE WELL & 282121082071101 & - & Pasco & 282122 & 820710 & $5 / 17 / 2010$ & 73 & \\
\hline FL & USGS-FLWSC & $\begin{array}{l}\text { Cypress Creek 6" UFA \#3 near } \\
\text { San Antonio }\end{array}$ & 281622082241301 & - & Pasco & 281623 & 822412 & $5 / 24 / 2010$ & 54 & \\
\hline FL & USGS-FLWSC & $\begin{array}{l}\text { Cypress Creek Deep Well } \\
\text { E-107 near Drexel, FL }\end{array}$ & 281801082225101 & - & Pasco & 281802 & 822252 & $5 / 21 / 2010$ & 47 & \\
\hline FL & USGS-FLWSC & $\begin{array}{l}\text { Cypress Creek TMR-4 UFA } \\
\text { near San Antonio }\end{array}$ & 281650082244501 & - & Pasco & 281651 & 822444 & $5 / 21 / 2010$ & 55 & \\
\hline FL & USGS-FLWSC & $\begin{array}{l}\text { Cypress Creek UFA \#TMR-5 } \\
\text { near San Antonio }\end{array}$ & 281535082241301 & - & Pasco & 281536 & 822412 & $5 / 21 / 2010$ & 55 & \\
\hline FL & USGS-FLWSC & $\begin{array}{l}\text { D.A. Sutyak 6" UFA near Ma- } \\
\text { saryktown }\end{array}$ & 282434082283601 & - & Pasco & 282435 & 822835 & $5 / 17 / 2010$ & 24 & \\
\hline FL & USGS-FLWSC & $\begin{array}{l}\text { D-0115 J-0179 UFA VILLAGE } \\
\text { DANCE CTR }\end{array}$ & 301617081421601 & - & Duval & 301618 & 814215 & $5 / 19 / 2010$ & 30 & \\
\hline
\end{tabular}


Table 1. Water-level measurements in Florida and parts of Georgia, South Carolina, and Alabama, May-June 2010.-Continued

[NGVD 29, National Geodetic Vertical Datum of 1929. Abbreviations for agency database: ACEPD, Alachua County Environmental Protection Department; FGS, Florida Geological Survey; GSA, Geological Survey of Alabama; NWFWMD, Northwest Florida Water Management District; SFWMD, South Florida Water Management District; SRWMD, Suwannee River Water Management District; USGSALWSC, U.S. Geological Survey Alabama Water Science Center; USGS-FLWSC, U.S. Geological Survey Florida Water Science Center; GAWSC, U.S. Geological Survey Georgia Water Science Center; SCWSC, U.S. Geological Survey South Carolina Water Science Center. Abbreviations for Station names: BLVD., boulevard; CO, county; CR, county road; E, east; HS, high school; IFAS, Institute of Food and Agricultural Services; LK, lake; MW, monitoring well; N, north; NE, northeast; NE, northeast; NF, national forest; NW, northwest; PK, park; R., river; RD., road; SE, southeast; SE, southeast; SR, state road; ST., street; TWR, tower; UFA, Upper Floridan aquifer; - - not available; \#, number; “, inches]

\begin{tabular}{|c|c|c|c|c|c|c|c|c|c|c|}
\hline State & $\begin{array}{l}\text { Agency } \\
\text { database }\end{array}$ & Station name & $\begin{array}{c}\text { USGS site } \\
\text { identification } \\
\text { number }\end{array}$ & $\begin{array}{l}\text { Local agency } \\
\text { unique site } \\
\text { identifier }\end{array}$ & County & $\begin{array}{l}\text { Latitude } \\
\text { (degree } \\
\text { minutes } \\
\text { and } \\
\text { seconds) } \\
\text { [DDMMSS] }\end{array}$ & $\begin{array}{l}\text { Longitude } \\
\text { (degree } \\
\text { minutes } \\
\text { and } \\
\text { seconds) } \\
\text { [DDMMSS] }\end{array}$ & $\begin{array}{c}\text { Date of } \\
\text { measure- } \\
\text { ment }\end{array}$ & $\begin{array}{c}\text { Water } \\
\text { level } \\
\text { altitude } \\
\text { (feet } \\
\text { above } \\
\text { NGVD 29) }\end{array}$ & Remarks \\
\hline FL & USGS-FLWSC & $\begin{array}{l}\text { D-0160 (City of) Neptune } \\
\text { Beach 4" UFA Artesian, } \\
\text { Neptune Beac }\end{array}$ & 301852081234201 & - & Duval & 301853 & 812341 & $5 / 19 / 2010$ & 25 & \\
\hline FL & USGS-FLWSC & $\begin{array}{l}\text { D-0221 ROLLING } \\
\text { HILLS;USGS \#D-589 }\end{array}$ & 301758081462901 & - & Duval & 301759 & 814628 & $5 / 18 / 2010$ & 46 & \\
\hline FL & USGS-FLWSC & $\begin{array}{l}\text { D-0254 J-0321 BALDWIN RR } \\
\text { YARD UFA }\end{array}$ & 301725081584501 & - & Duval & 301726 & 815844 & $5 / 17 / 2010$ & 49 & \\
\hline FL & USGS-FLWSC & $\begin{array}{l}\text { D-0259 BEACH HAVEN } \\
\text { WELL NR JACKSON- } \\
\text { VILLE BEACH }\end{array}$ & 301652081265001 & - & Duval & 301653 & 812648 & $5 / 19 / 2010$ & 16 & \\
\hline FL & USGS-FLWSC & $\begin{array}{l}\text { D-0264 J-0030 SMURFIT- } \\
\text { STONE CONTAINER } \\
\text { CORP UFA }\end{array}$ & 302608081354903 & - & Duval & 302609 & 813548 & $5 / 18 / 2010$ & 36 & \\
\hline FL & USGS-FLWSC & $\begin{array}{l}\text { D-0348 J-0413 GARDEN ST } \\
\text { UFA }\end{array}$ & 302416081522601 & - & Duval & 302417 & 815225 & $5 / 18 / 2010$ & 40 & \\
\hline FL & USGS-FLWSC & $\begin{array}{l}\text { D-0395 J-0462 LITTLE } \\
\text { TALBOT ISLAND UFA AT } \\
\text { PARK }\end{array}$ & 302724081244801 & - & Duval & 302725 & 812447 & $5 / 18 / 2010$ & 34 & \\
\hline FL & USGS-FLWSC & $\begin{array}{l}\text { D-0401 J-0468 DUVAL CO } \\
\text { PRISON FARM UFA }\end{array}$ & 303216081433301 & - & Duval & 303217 & 814332 & $5 / 19 / 2010$ & 36 & \\
\hline FL & USGS-FLWSC & $\begin{array}{l}\text { D-0424 NR FT CAROLINE } \\
\text { PARK AT SPANISH POND }\end{array}$ & 302307081293801 & - & Duval & 302308 & 812937 & $5 / 19 / 2010$ & 28 & \\
\hline FL & USGS-FLWSC & $\begin{array}{l}\text { D-0464A JAX CITY UFA } 1459 \\
\text { ROXIE ST, MAYPORT }\end{array}$ & 302339081254702 & - & Duval & 302340 & 812546 & $5 / 19 / 2010$ & 29 & \\
\hline FL & USGS-FLWSC & $\begin{array}{l}\text { D-0547 SSIDE TOWER UFA } \\
\text { (USGS D-3824) }\end{array}$ & 301710081323603 & - & Duval & 301711 & 813235 & $5 / 19 / 2010$ & 17 & \\
\hline FL & USGS-FLWSC & D-0667 (D-122A) J-0186 UFA & 302304081383202 & - & Duval & 302305 & 813831 & $5 / 18 / 2010$ & 36 & \\
\hline
\end{tabular}


Table 1. Water-level measurements in Florida and parts of Georgia, South Carolina, and Alabama, May-June 2010.—Continued

[NGVD 29, National Geodetic Vertical Datum of 1929. Abbreviations for agency database: ACEPD, Alachua County Environmental Protection Department; FGS, Florida Geological Survey; GSA, Geological Survey of Alabama; NWFWMD, Northwest Florida Water Management District; SFWMD, South Florida Water Management District; SRWMD, Suwannee River Water Management District; USGSALWSC, U.S. Geological Survey Alabama Water Science Center; USGS-FLWSC, U.S. Geological Survey Florida Water Science Center; GAWSC, U.S. Geological Survey Georgia Water Science Center; SCWSC, U.S. Geological Survey South Carolina Water Science Center. Abbreviations for Station names: BLVD., boulevard; CO, county; CR, county road; E, east; HS, high school; IFAS, Institute of Food and Agricultural Services; LK, lake; MW, monitoring well; N, north; NE, northeast; NE, northeast; NF, national forest; NW, northwest; PK, park; R., river; RD., road; SE, southeast; SE, southeast; SR, state road; ST., street; TWR, tower; UFA, Upper Floridan aquifer; —, not available; \#, number; “, inches]

\begin{tabular}{|c|c|c|c|c|c|c|c|c|c|c|}
\hline State & $\begin{array}{l}\text { Agency } \\
\text { database }\end{array}$ & Station name & $\begin{array}{c}\text { USGS site } \\
\text { identification } \\
\text { number }\end{array}$ & $\begin{array}{l}\text { Local agency } \\
\text { unique site } \\
\text { identifier }\end{array}$ & County & $\begin{array}{l}\text { Latitude } \\
\text { (degree } \\
\text { minutes } \\
\text { and } \\
\text { seconds) } \\
\text { [DDMMSS] }\end{array}$ & $\begin{array}{l}\text { Longitude } \\
\text { (degree } \\
\text { minutes } \\
\text { and } \\
\text { seconds) } \\
\text { [DDMMSS] }\end{array}$ & $\begin{array}{c}\text { Date of } \\
\text { measure- } \\
\text { ment }\end{array}$ & $\begin{array}{c}\text { Water } \\
\text { level } \\
\text { altitude } \\
\text { (feet } \\
\text { above } \\
\text { NGVD 29) }\end{array}$ & Remarks \\
\hline FL & USGS-FLWSC & $\begin{array}{l}\text { D-0673; (D-124)TISONIA } \\
\text { FORESTRY FIRETOWER } \\
\text { UFA }\end{array}$ & 303209081371801 & - & Duval & 302114 & 813116 & $5 / 18 / 2010$ & 33 & \\
\hline $\mathrm{FL}$ & USGS-FLWSC & $\begin{array}{l}\text { D-1307 CAMDEN RD MONI- } \\
\text { TOR WELL }\end{array}$ & 302709081311601 & - & Duval & 302710 & 813115 & $5 / 18 / 2010$ & 36 & \\
\hline FL & USGS-FLWSC & $\begin{array}{l}\text { D-1309 (USGS D-751) Dins- } \\
\text { more Elem School 6" UFA }\end{array}$ & 302521081455601 & - & Duval & 302532 & 814555 & $5 / 18 / 2010$ & 47 & \\
\hline FL & USGS-FLWSC & $\begin{array}{l}\text { D-1313 GREENLAND PINES } \\
\text { UFA }\end{array}$ & 300926081343002 & - & Duval & 300927 & 813429 & $5 / 18 / 2010$ & 32 & \\
\hline FL & USGS-FLWSC & $\begin{array}{l}\text { D-1350 9A MITIGATION } \\
\text { PARCEL "DEER CREEK" }\end{array}$ & 301129081311301 & - & Duval & 301129 & 813113 & $5 / 19 / 2010$ & 29 & \\
\hline FL & USGS-FLWSC & $\begin{array}{l}\text { D-1394 NAS WELL NR } \\
\text { JACKSONVILLE }\end{array}$ & 301347081421801 & - & Duval & 301347 & 814217 & $5 / 19 / 2010$ & 29 & \\
\hline FL & USGS-FLWSC & $\begin{array}{l}\text { D-1782 J-1819 JAX CITY UFA } \\
\text { \#5 RIVER OAKS }\end{array}$ & 301749081384602 & - & Duval & 301750 & 813845 & $5 / 18 / 2010$ & 40 & \\
\hline FL & USGS-FLWSC & Days Inn 10" UFA near Seffner & 280004082181601 & - & Hillsborough & 280005 & 821815 & $5 / 19 / 2010$ & 19 & \\
\hline FL & USGS-FLWSC & Debuel Rd 6" UFA near Lutz & 280740082271001 & - & Hillsborough & 280741 & 822709 & $5 / 18 / 2010$ & 55 & \\
\hline FL & USGS-FLWSC & DELMAS NIX & 283613082184301 & - & Hernando & 283614 & 821842 & $5 / 18 / 2010$ & 34 & \\
\hline FL & USGS-FLWSC & $\begin{array}{l}\text { Deseret Ranch Well No. } 3 \text { near } \\
\text { Kenansville }\end{array}$ & 275629080504901 & - & Brevard & 275630 & 805048 & $5 / 19 / 2010$ & 39 & \\
\hline FL & USGS-FLWSC & $\begin{array}{l}\text { DEVILS DEN SINK CE-08 } \\
\text { NR WILLISTON }\end{array}$ & 292430082283001 & - & Levy & 292431 & 822829 & $5 / 26 / 2010$ & 45 & \\
\hline FL & USGS-FLWSC & DICK SURRENCY WELL & 293723082120102 & - & Alachua & 293724 & 821200 & $5 / 25 / 2010$ & 76 & \\
\hline FL & USGS-FLWSC & $\begin{array}{l}\text { DIXIE LIME 4" UFA, LEBA- } \\
\text { NON STATION }\end{array}$ & 291004082382901 & - & Levy & 291005 & 823828 & $5 / 26 / 2010$ & 24 & \\
\hline
\end{tabular}


Table 1. Water-level measurements in Florida and parts of Georgia, South Carolina, and Alabama, May-June 2010.-Continued

[NGVD 29, National Geodetic Vertical Datum of 1929. Abbreviations for agency database: ACEPD, Alachua County Environmental Protection Department; FGS, Florida Geological Survey; GSA, Geological Survey of Alabama; NWFWMD, Northwest Florida Water Management District; SFWMD, South Florida Water Management District; SRWMD, Suwannee River Water Management District; USGSALWSC, U.S. Geological Survey Alabama Water Science Center; USGS-FLWSC, U.S. Geological Survey Florida Water Science Center; GAWSC, U.S. Geological Survey Georgia Water Science Center; SCWSC, U.S. Geological Survey South Carolina Water Science Center. Abbreviations for Station names: BLVD., boulevard; CO, county; CR, county road; E, east; HS, high school; IFAS, Institute of Food and Agricultural Services; LK, lake; MW, monitoring well; N, north; NE, northeast; NE, northeast; NF, national forest; NW, northwest; PK, park; R., river; RD., road; SE, southeast; SE, southeast; SR, state road; ST., street; TWR, tower; UFA, Upper Floridan aquifer; - - not available; \#, number; “, inches]

\begin{tabular}{|c|c|c|c|c|c|c|c|c|c|c|}
\hline State & $\begin{array}{c}\text { Agency } \\
\text { database }\end{array}$ & Station name & $\begin{array}{c}\text { USGS site } \\
\text { identification } \\
\text { number }\end{array}$ & $\begin{array}{l}\text { Local agency } \\
\text { unique site } \\
\text { identifier }\end{array}$ & County & $\begin{array}{l}\text { Latitude } \\
\text { (degree } \\
\text { minutes } \\
\text { and } \\
\text { seconds) } \\
\text { [DDMMSS] }\end{array}$ & $\begin{array}{l}\text { Longitude } \\
\text { (degree } \\
\text { minutes } \\
\text { and } \\
\text { seconds) } \\
\text { ] [DDMMSS] }\end{array}$ & $\begin{array}{c}\text { Date of } \\
\text { measure- } \\
\text { ment }\end{array}$ & $\begin{array}{l}\text { Water } \\
\text { level } \\
\text { altitude } \\
\text { (feet } \\
\text { above } \\
\text { NGVD 29) }\end{array}$ & Remarks \\
\hline FL & USGS-FLWSC & $\begin{array}{l}\text { DOF-BUREAU OF PROPER- } \\
\text { TY ASSISTANCE 4" UFA, } \\
\text { STARKE }\end{array}$ & 295502082074701 & -072205001 & Bradford & 295503 & 820746 & $5 / 18 / 2010$ & 62 & $\begin{array}{l}\text { SRWMD Site iden- } \\
\text { tification number } \\
-072205001\end{array}$ \\
\hline FL & USGS-FLWSC & $\begin{array}{l}\text { DOT HY } 41 \text { OBS WELL AT } \\
\text { INVERNESS }\end{array}$ & 285102082204001 & - & Citrus & 285103 & 822039 & $5 / 19 / 2010$ & 28 & \\
\hline FL & USGS-FLWSC & $\begin{array}{l}\text { DR. PHILLIPS \& SONS, INC. } \\
\text { UFA NR MASCOTTE }\end{array}$ & 283530081514501 & - & Lake & 283531 & 815144 & $5 / 19 / 2010$ & 88 & \\
\hline FL & USGS-FLWSC & $\begin{array}{l}\text { DUDA RANCH L-2; (BR } \\
\text { 1842) }\end{array}$ & 281210080473001 & - & Brevard & 281211 & 804729 & $5 / 24 / 2010$ & 37 & \\
\hline FL & USGS-FLWSC & $\begin{array}{l}\text { Dunedin Prod 10" UFA \#10A, } \\
\text { Dunedin }\end{array}$ & 280134082454801 & - & Pinellas & 280135 & 824547 & $5 / 18 / 2010$ & 5 & \\
\hline FL & USGS-FLWSC & $\begin{array}{l}\text { DUVAL ISLAND WELL NR } \\
\text { FLORAL CITY }\end{array}$ & 284609082163001 & - & Citrus & 284610 & 821629 & $5 / 19 / 2010$ & 40 & \\
\hline FL & USGS-FLWSC & $\begin{array}{l}\text { E Lake 12" UFA \#17 near } \\
\text { Tarpon Springs }\end{array}$ & 280446082390701 & - & Pinellas & 280447 & 823906 & $5 / 19 / 2010$ & 17 & \\
\hline FL & USGS-FLWSC & E LAKE WALES UTILITY & 275137081252501 & - & Polk & 275138 & 812524 & $5 / 18 / 2010$ & 80 & \\
\hline FL & USGS-FLWSC & $\begin{array}{l}\text { E Manatee Co. 2" UFA near } \\
\text { Duette }\end{array}$ & 273255082072601 & - & Manatee & 273256 & 820725 & $5 / 18 / 2010$ & 20 & \\
\hline FL & USGS-FLWSC & E12 334 GATORAMA & 265452081165401 & - & Glades & 265453 & 811653 & $5 / 18 / 2010$ & 50 & $\begin{array}{l}\text { Uncorrected hydrau- } \\
\text { lic head reported; } \\
\text { brackish to saline } \\
\text { water; not con- } \\
\text { toured on Potentio- } \\
\text { metric surface map }\end{array}$ \\
\hline FL & USGS-FLWSC & $\begin{array}{l}\text { EAST LAKE DP WELL } 14 \text { NR } \\
\text { TARPON SPRGS }\end{array}$ & 280546082390701 & - & Pinellas & 280547 & 823906 & $5 / 19 / 2010$ & 19 & \\
\hline FL & USGS-FLWSC & $\begin{array}{l}\text { Edgeville UFA \#3 4" UFA near } \\
\text { Edgeville }\end{array}$ & 271832082064801 & - & Manatee & 271833 & 820647 & $5 / 18 / 2010$ & 20 & \\
\hline
\end{tabular}


Table 1. Water-level measurements in Florida and parts of Georgia, South Carolina, and Alabama, May-June 2010.—Continued

[NGVD 29, National Geodetic Vertical Datum of 1929. Abbreviations for agency database: ACEPD, Alachua County Environmental Protection Department; FGS, Florida Geological Survey; GSA, Geological Survey of Alabama; NWFWMD, Northwest Florida Water Management District; SFWMD, South Florida Water Management District; SRWMD, Suwannee River Water Management District; USGSALWSC, U.S. Geological Survey Alabama Water Science Center; USGS-FLWSC, U.S. Geological Survey Florida Water Science Center; GAWSC, U.S. Geological Survey Georgia Water Science Center; SCWSC, U.S. Geological Survey South Carolina Water Science Center. Abbreviations for Station names: BLVD., boulevard; CO, county; CR, county road; E, east; HS, high school; IFAS, Institute of Food and Agricultural Services; LK, lake; MW, monitoring well; N, north; NE, northeast; NE, northeast; NF, national forest; NW, northwest; PK, park; R., river; RD., road; SE, southeast; SE, southeast; SR, state road; ST., street; TWR, tower; UFA, Upper Floridan aquifer; —, not available; \#, number; “, inches]

\begin{tabular}{|c|c|c|c|c|c|c|c|c|c|c|}
\hline State & $\begin{array}{l}\text { Agency } \\
\text { database }\end{array}$ & Station name & $\begin{array}{c}\text { USGS site } \\
\text { identification } \\
\text { number }\end{array}$ & $\begin{array}{l}\text { Local agency } \\
\text { unique site } \\
\text { identifier }\end{array}$ & County & $\begin{array}{l}\text { Latitude } \\
\text { (degree } \\
\text { minutes } \\
\text { and } \\
\text { seconds) } \\
\text { [DDMMSS] }\end{array}$ & $\begin{array}{l}\text { Longitude } \\
\text { (degree } \\
\text { minutes } \\
\text { and } \\
\text { seconds) } \\
\text { [DDMMSS] }\end{array}$ & $\begin{array}{c}\text { Date of } \\
\text { measure- } \\
\text { ment }\end{array}$ & $\begin{array}{c}\text { Water } \\
\text { level } \\
\text { altitude } \\
\text { (feet } \\
\text { above } \\
\text { NGVD 29) }\end{array}$ & Remarks \\
\hline FL & USGS-FLWSC & $\begin{array}{l}\text { Edna Kendall Irrigation 6" UFA } \\
\text { near Deanville }\end{array}$ & 291955081200901 & - & Flagler & 291956 & 812008 & $5 / 18 / 2010$ & 12 & \\
\hline FL & USGS-FLWSC & $\begin{array}{l}\text { Eldridge-Wilde 12" UFA } \\
\text { \#2South near Tarpon Springs }\end{array}$ & 280856082401201 & - & Pinellas & 280857 & 824011 & $5 / 20 / 2010$ & 19 & \\
\hline FL & USGS-FLWSC & $\begin{array}{l}\text { Eldridge-Wilde 6" UFA \#N-1 } \\
\text { near Tarpon Springs }\end{array}$ & 280942082390601 & - & Pinellas & 280943 & 823905 & $5 / 20 / 2010$ & 18 & \\
\hline FL & USGS-FLWSC & $\begin{array}{l}\text { Eldridge-Wilde 6" UFA \#N-4 } \\
\text { near Tarpon Springs }\end{array}$ & 280944082380501 & - & Hillsborough & 280945 & 823804 & $5 / 20 / 2010$ & 29 & \\
\hline FL & USGS-FLWSC & $\begin{array}{l}\text { Eldridge-Wilde Dp Well N3 Nr } \\
\text { Tarpon Sgs }\end{array}$ & 281022082400201 & - & Pasco & 281023 & 824002 & $5 / 20 / 2010$ & 20 & \\
\hline FL & USGS-FLWSC & $\begin{array}{l}\text { ELMER HEATH SR581 N OF } \\
\text { INVERNESS }\end{array}$ & 285248082183201 & - & Citrus & 285249 & 821831 & $5 / 19 / 2010$ & 38 & \\
\hline FL & USGS-FLWSC & $\begin{array}{l}\text { F-0087 USGS UFA FLAG14 } \\
\text { AT BUNNELL }\end{array}$ & 292750081152001 & - & Flagler & 292751 & 811519 & $5 / 18 / 2010$ & 16 & \\
\hline FL & USGS-FLWSC & $\begin{array}{l}\text { F-0126 George Allen Estate 6" } \\
\text { UFA }\end{array}$ & 292647081182001 & - & Flagler & 292648 & 811819 & $5 / 18 / 2010$ & 9 & \\
\hline FL & USGS-FLWSC & $\begin{array}{l}\text { F-0158 SJRWMD UFA ITT } \\
\text { PALM COAST }\end{array}$ & 293313081132402 & - & Flagler & 293314 & 811323 & $5 / 18 / 2010$ & 14 & \\
\hline FL & USGS-FLWSC & $\begin{array}{l}\text { F-0165 SJRWMD UFA ITT } \\
\text { PALM COAST LW-20 N } \\
\text { BOUND. }\end{array}$ & 293529081191701 & - & Flagler & 293530 & 811916 & $5 / 18 / 2010$ & 15 & \\
\hline FL & USGS-FLWSC & F-0176 UFA BULLOW RUINS & 292603081082502 & - & Flagler & 292604 & 810824 & $5 / 18 / 2010$ & 8 & \\
\hline FL & USGS-FLWSC & $\begin{array}{l}\text { F-0179 SR11 NORTH OF } \\
\text { CODYS CORNER }\end{array}$ & 292445081181801 & - & Flagler & 292445 & 811818 & $5 / 18 / 2010$ & 9 & \\
\hline FL & USGS-FLWSC & $\begin{array}{l}\text { F-0200 WASHINGTON OAKS } \\
\text { PK MET SITE UFA }\end{array}$ & 293754081121901 & - & Flagler & 293755 & 811218 & $5 / 17 / 2010$ & 15 & \\
\hline
\end{tabular}


Table 1. Water-level measurements in Florida and parts of Georgia, South Carolina, and Alabama, May-June 2010.—Continued

[NGVD 29, National Geodetic Vertical Datum of 1929. Abbreviations for agency database: ACEPD, Alachua County Environmental Protection Department; FGS, Florida Geological Survey; GSA, Geological Survey of Alabama; NWFWMD, Northwest Florida Water Management District; SFWMD, South Florida Water Management District; SRWMD, Suwannee River Water Management District; USGSALWSC, U.S. Geological Survey Alabama Water Science Center; USGS-FLWSC, U.S. Geological Survey Florida Water Science Center; GAWSC, U.S. Geological Survey Georgia Water Science Center; SCWSC, U.S. Geological Survey South Carolina Water Science Center. Abbreviations for Station names: BLVD., boulevard; CO, county; CR, county road; E, east; HS, high school; IFAS, Institute of Food and Agricultural Services; LK, lake; MW, monitoring well; N, north; NE, northeast; NE, northeast; NF, national forest; NW, northwest; PK, park; R., river; RD., road; SE, southeast; SE, southeast; SR, state road; ST., street; TWR, tower; UFA, Upper Floridan aquifer; —, not available; \#, number; “, inches]

\begin{tabular}{|c|c|c|c|c|c|c|c|c|c|c|}
\hline State & $\begin{array}{l}\text { Agency } \\
\text { database }\end{array}$ & Station name & $\begin{array}{c}\text { USGS site } \\
\text { identification } \\
\text { number }\end{array}$ & $\begin{array}{c}\text { Local agency } \\
\text { unique site } \\
\text { identifier }\end{array}$ & County & $\begin{array}{l}\text { Latitude } \\
\text { (degree } \\
\text { minutes } \\
\text { and } \\
\text { seconds) } \\
\text { [DDMMSS] }\end{array}$ & $\begin{array}{l}\text { Longitude } \\
\text { (degree } \\
\text { minutes } \\
\text { and } \\
\text { seconds) } \\
\text { [DDMMSS] }\end{array}$ & $\begin{array}{c}\text { Date of } \\
\text { measure- } \\
\text { ment }\end{array}$ & $\begin{array}{c}\text { Water } \\
\text { level } \\
\text { altitude } \\
\text { (feet } \\
\text { above } \\
\text { NGVD 29) }\end{array}$ & Remarks \\
\hline FL & USGS-FLWSC & $\begin{array}{l}\text { F-0225 LENSSEN 4" UFA, } \\
\text { BEVERLY BEACH }\end{array}$ & 293128081090501 & - & Flagler & 293129 & 810904 & $5 / 18 / 2010$ & 6 & \\
\hline FL & USGS-FLWSC & F-0251 RELAY TOWER UFA & 291818081190401 & - & Flagler & 291819 & 811903 & $5 / 18 / 2010$ & 17 & \\
\hline FL & USGS-FLWSC & $\begin{array}{l}\text { F-0257 STRAWN WELL NR } \\
\text { DEANVILLE }\end{array}$ & 291913081224201 & - & Flagler & 291914 & 812241 & $5 / 18 / 2010$ & 17 & \\
\hline FL & USGS-FLWSC & F-0294 UFA TIGER ISLAND & 293344081232401 & - & Flagler & 293345 & 812323 & $5 / 18 / 2010$ & 15 & \\
\hline FL & USGS-FLWSC & $\begin{array}{l}\text { F-0353 WESTSIDE BAPTIST } \\
\text { UFA NR BIMINI }\end{array}$ & 292757081222801 & - & Flagler & 292758 & 812227 & $5 / 18 / 2010$ & 10 & \\
\hline FL & USGS-FLWSC & $\begin{array}{l}\text { FERRIS PKG CO WELL AT } \\
\text { FLORAL CTY }\end{array}$ & 284508082174601 & - & Citrus & 284509 & 821745 & $5 / 19 / 2010$ & 33 & \\
\hline FL & USGS-FLWSC & $\begin{array}{l}\text { FIRE TWR UFA CE73 AT } \\
\text { DUNNELLON }\end{array}$ & 290306082232802 & - & Marion & 290307 & 822327 & $5 / 24 / 2010$ & 55 & \\
\hline FL & USGS-FLWSC & $\begin{array}{l}\text { FISH LAKE DP WELL NR } \\
\text { LAKELAND }\end{array}$ & 280503081552801 & - & Polk & 280504 & 815527 & $5 / 19 / 2010$ & 111 & \\
\hline FL & USGS-FLWSC & $\begin{array}{l}\text { Fisher 6" UFA \#60 near } \\
\text { Knights }\end{array}$ & 280350082104401 & - & Hillsborough & 280351 & 821043 & $5 / 17 / 2010$ & 82 & \\
\hline FL & USGS-FLWSC & Fl-2-1000 8" UFA near Lutz & 281325082215301 & - & Pasco & 281326 & 822152 & $5 / 24 / 2010$ & 57 & \\
\hline FL & USGS-FLWSC & FP\&L 12" UFA, Piney Point & 273718082315501 & - & Manatee & 273719 & 823154 & $5 / 20 / 2010$ & 13 & \\
\hline FL & USGS-FLWSC & $\begin{array}{l}\text { FPC 4" UFA \#1 near Tarpon } \\
\quad \text { Springs }\end{array}$ & 281046082470801 & - & Pasco & 281047 & 824707 & $5 / 19 / 2010$ & 2 & \\
\hline FL & USGS-FLWSC & $\begin{array}{c}\text { FRANKS PLACE N-0119 UFA } \\
\text { (N-0100 REPLACEMENT) }\end{array}$ & 303357081295601 & - & Nassau & 303358 & 812955 & $5 / 17 / 2010$ & 26 & \\
\hline FL & USGS-FLWSC & $\begin{array}{l}\text { Ft. Desoto 4" UFA near Ft. } \\
\text { Desoto Park }\end{array}$ & 273655082440901 & - & Pinellas & 273656 & 824408 & $5 / 17 / 2010$ & 11 & \\
\hline FL & USGS-FLWSC & $\begin{array}{l}\text { Ft. Green Springs Rd 6" UFA } \\
\text { near Bradley Junction }\end{array}$ & 274155081573201 & - & Polk & 274156 & 815731 & $5 / 19 / 2010$ & 65 & \\
\hline
\end{tabular}


Table 1. Water-level measurements in Florida and parts of Georgia, South Carolina, and Alabama, May-June 2010.—Continued

[NGVD 29, National Geodetic Vertical Datum of 1929. Abbreviations for agency database: ACEPD, Alachua County Environmental Protection Department; FGS, Florida Geological Survey; GSA, Geological Survey of Alabama; NWFWMD, Northwest Florida Water Management District; SFWMD, South Florida Water Management District; SRWMD, Suwannee River Water Management District; USGSALWSC, U.S. Geological Survey Alabama Water Science Center; USGS-FLWSC, U.S. Geological Survey Florida Water Science Center; GAWSC, U.S. Geological Survey Georgia Water Science Center; SCWSC, U.S. Geological Survey South Carolina Water Science Center. Abbreviations for Station names: BLVD., boulevard; CO, county; CR, county road; E, east; HS, high school; IFAS, Institute of Food and Agricultural Services; LK, lake; MW, monitoring well; N, north; NE, northeast; NE, northeast; NF, national forest; NW, northwest; PK, park; R., river; RD., road; SE, southeast; SE, southeast; SR, state road; ST., street; TWR, tower; UFA, Upper Floridan aquifer; —, not available; \#, number; “, inches]

\begin{tabular}{|c|c|c|c|c|c|c|c|c|c|c|}
\hline State & $\begin{array}{l}\text { Agency } \\
\text { database }\end{array}$ & Station name & $\begin{array}{c}\text { USGS site } \\
\text { identification } \\
\text { number }\end{array}$ & $\begin{array}{l}\text { Local agency } \\
\text { unique site } \\
\text { identifier }\end{array}$ & County & $\begin{array}{l}\text { Latitude } \\
\text { (degree } \\
\text { minutes } \\
\text { and } \\
\text { seconds) } \\
\text { [DDMMSS] }\end{array}$ & $\begin{array}{l}\text { Longitude } \\
\text { (degree } \\
\text { minutes } \\
\text { and } \\
\text { seconds) } \\
\text { ] [DDMMSS] }\end{array}$ & $\begin{array}{c}\text { Date of } \\
\text { measure- } \\
\text { ment }\end{array}$ & $\begin{array}{c}\text { Water } \\
\text { level } \\
\text { altitude } \\
\text { (feet } \\
\text { above } \\
\text { NGVD 29) }\end{array}$ & Remarks \\
\hline FL & USGS-FLWSC & $\begin{array}{l}\text { G.N. SMITH WEST OF OX- } \\
\text { FORD }\end{array}$ & 285536082044001 & - & Sumter & 285537 & 820439 & $5 / 19 / 2010$ & 49 & \\
\hline FL & USGS-FLWSC & $\begin{array}{l}\text { Garden St. Triangle 10" UFA, } \\
\text { Clearwater }\end{array}$ & 275843082474201 & - & Pinellas & 275844 & 824741 & $5 / 18 / 2010$ & 5 & \\
\hline FL & USGS-FLWSC & $\begin{array}{l}\text { Gardinier 8" UFA near Bowling } \\
\text { Green }\end{array}$ & 274151081513201 & - & Polk & 274152 & 815131 & $5 / 19 / 2010$ & 66 & \\
\hline FL & USGS-FLWSC & $\begin{array}{l}\text { Gen Dev 12" UFA \#M-2 near } \\
\text { Ft. Ogden }\end{array}$ & 270540082001101 & - & De Soto & 270541 & 820010 & $5 / 19 / 2010$ & 38 & \\
\hline FL & USGS-FLWSC & $\begin{array}{l}\text { GOETHE ROAD 2" UFA, LCR } \\
\quad 320\end{array}$ & 290605082372601 & - & Levy & 290606 & 823725 & $5 / 26 / 2010$ & 27 & \\
\hline FL & USGS-FLWSC & Gough UFA near Myakka Head & 272537082033301 & - & Manatee & 272538 & 820332 & $5 / 17 / 2010$ & 13 & \\
\hline FL & USGS-FLWSC & $\begin{array}{l}\text { GREEN SWP WELL L11MD } \\
\text { NR DADE CITY }\end{array}$ & 281951082012001 & - & Sumter & 281952 & 820119 & $5 / 17 / 2010$ & 90 & \\
\hline FL & USGS-FLWSC & $\begin{array}{l}\text { GREEN SWP WELL L12BD } \\
\text { NR BAY LAKE }\end{array}$ & 282740082012101 & - & Sumter & 282741 & 820120 & $5 / 17 / 2010$ & 91 & \\
\hline FL & USGS-FLWSC & $\begin{array}{l}\text { H. Kent Grove 10" UFA near } \\
\text { Gowers Corner }\end{array}$ & 282044082312401 & - & Pasco & 282045 & 823123 & $5 / 18 / 2010$ & 59 & \\
\hline FL & USGS-FLWSC & $\begin{array}{l}\text { Hagstrom (Albin \& Sons) 6" } \\
\text { UFA, W Of DeLeon Springs }\end{array}$ & 290737081220301 & - & Volusia & 290738 & 812202 & $5 / 26 / 2010$ & 8 & \\
\hline FL & USGS-FLWSC & $\begin{array}{l}\text { Harper 8" UFA, E of Murphy } \\
\text { Rd }\end{array}$ & 291150081282501 & - & Volusia & 291151 & 812824 & $5 / 26 / 2010$ & 28 & \\
\hline FL & USGS-FLWSC & $\begin{array}{l}\text { HAYCRAFT WELL NEAR } \\
\text { DADE CITY }\end{array}$ & 282154082142401 & - & Pasco & 282155 & 821423 & $5 / 17 / 2010$ & 66 & \\
\hline FL & USGS-FLWSC & $\begin{array}{l}\text { Henderson 6" UFA near } \\
\text { Bethany }\end{array}$ & 272823082182701 & - & Manatee & 272824 & 821826 & $5 / 17 / 2010$ & -7 & \\
\hline FL & USGS-FLWSC & $\begin{array}{l}\text { HERSHEL KYPER SOUTH } \\
\text { OF ROMEO }\end{array}$ & 291056082263201 & - & Marion & 291050 & 822632 & $5 / 24 / 2010$ & 39 & \\
\hline
\end{tabular}


Table 1. Water-level measurements in Florida and parts of Georgia, South Carolina, and Alabama, May-June 2010.-Continued

[NGVD 29, National Geodetic Vertical Datum of 1929. Abbreviations for agency database: ACEPD, Alachua County Environmental Protection Department; FGS, Florida Geological Survey; GSA, Geological Survey of Alabama; NWFWMD, Northwest Florida Water Management District; SFWMD, South Florida Water Management District; SRWMD, Suwannee River Water Management District; USGSALWSC, U.S. Geological Survey Alabama Water Science Center; USGS-FLWSC, U.S. Geological Survey Florida Water Science Center; GAWSC, U.S. Geological Survey Georgia Water Science Center; SCWSC, U.S. Geological Survey South Carolina Water Science Center. Abbreviations for Station names: BLVD., boulevard; CO, county; CR, county road; E, east; HS, high school; IFAS, Institute of Food and Agricultural Services; LK, lake; MW, monitoring well; N, north; NE, northeast; NE, northeast; NF, national forest; NW, northwest; PK, park; R., river; RD., road; SE, southeast; SE, southeast; SR, state road; ST., street; TWR, tower; UFA, Upper Floridan aquifer; - - not available; \#, number; “, inches]

\begin{tabular}{|c|c|c|c|c|c|c|c|c|c|c|}
\hline State & $\begin{array}{l}\text { Agency } \\
\text { database }\end{array}$ & Station name & $\begin{array}{c}\text { USGS site } \\
\text { identification } \\
\text { number }\end{array}$ & $\begin{array}{l}\text { Local agency } \\
\text { unique site } \\
\text { identifier }\end{array}$ & County & $\begin{array}{l}\text { Latitude } \\
\text { (degree } \\
\text { minutes } \\
\text { and } \\
\text { seconds) } \\
\text { [DDMMSS] }\end{array}$ & $\begin{array}{l}\text { Longitude } \\
\text { (degree } \\
\text { minutes } \\
\text { and } \\
\text { seconds) } \\
\text { ] [DDMMSS] }\end{array}$ & $\begin{array}{c}\text { Date of } \\
\text { measure- } \\
\text { ment }\end{array}$ & $\begin{array}{l}\text { Water } \\
\text { level } \\
\text { altitude } \\
\text { (feet } \\
\text { above } \\
\text { NGVD 29) }\end{array}$ & Remarks \\
\hline FL & USGS-FLWSC & HIF-13 PHILLIP METZGER & 272512081122901 & - & Highlands & 272513 & 811228 & $5 / 18 / 2010$ & 49 & \\
\hline FL & USGS-FLWSC & HIF-23 GRAHAM CO DAIRY & 270627081313101 & - & Highlands & 270628 & 813130 & $5 / 17 / 2010$ & 48 & \\
\hline FL & USGS-FLWSC & HIF-26 J H HENDRIE DAIRY & 270556081204701 & - & Highlands & 270557 & 812046 & $5 / 17 / 2010$ & 47 & \\
\hline FL & USGS-FLWSC & HIF-5 CHARLES STIDHAM & 271134081234301 & - & Highlands & 271135 & 812342 & $5 / 18 / 2010$ & 48 & \\
\hline FL & USGS-FLWSC & $\begin{array}{l}\text { HIF-6 LYKES BROS 4IN } \\
\text { FLOW }\end{array}$ & 271456081074701 & - & Highlands & 271457 & 810746 & $5 / 18 / 2010$ & 42 & \\
\hline FL & USGS-FLWSC & HIF-8 BOX RANCH & 271306081284801 & - & Highlands & 271307 & 812847 & $5 / 18 / 2010$ & 47 & \\
\hline FL & USGS-FLWSC & $\begin{array}{l}\text { Highland Lakes Golf Course } \\
\text { FLDN Well near Palm Har- } \\
\text { bor, FL }\end{array}$ & 280501082430201 & - & Pinellas & 280500 & 824302 & $5 / 17 / 2010$ & 6 & \\
\hline FL & USGS-FLWSC & $\begin{array}{l}\text { HIGHLANDS VFD NR IN- } \\
\text { VERNESS }\end{array}$ & 284752082202501 & - & Citrus & 284753 & 822024 & $5 / 21 / 2010$ & 18 & \\
\hline FL & USGS-FLWSC & $\begin{array}{l}\text { Hillsborough Memorial Gar- } \\
\text { dens 10" UFA near Brandon }\end{array}$ & 275609082191401 & - & Hillsborough & 275610 & 821913 & $5 / 17 / 2010$ & 15 & \\
\hline FL & USGS-FLWSC & $\begin{array}{l}\text { Hillsborough St. Pk 4" UFA } \\
\text { near Zephyrhills }\end{array}$ & 280852082135601 & - & Hillsborough & 280853 & 821355 & $5 / 17 / 2010$ & 40 & \\
\hline FL & USGS-FLWSC & $\begin{array}{l}\text { HILOCHEE WMA 12" UFA, } \\
\text { Riddick Grove Rd (20-069- } \\
\text { 0091F) near Clermont }\end{array}$ & 282436081431201 & - & Lake & 282437 & 814311 & $5 / 20 / 2010$ & 115 & \\
\hline FL & USGS-FLWSC & $\begin{array}{l}\text { Hollis Ezel 4" UFA, SE Lake } \\
\text { George near Pierson }\end{array}$ & 291258081313701 & - & Volusia & 291259 & 813136 & $5 / 26 / 2010$ & 7 & \\
\hline FL & USGS-FLWSC & HOLOPAW TEST \#1 & 280826081031801 & - & Osceola & 280827 & 810317 & $5 / 18 / 2010$ & 42 & \\
\hline FL & USGS-FLWSC & $\begin{array}{l}\text { Homeland 24" UFA \#9 near } \\
\text { Homeland }\end{array}$ & 274841081480901 & - & Polk & 274842 & 814808 & $5 / 19 / 2010$ & 67 & \\
\hline FL & USGS-FLWSC & $\begin{array}{l}\text { Homosassa 4" UFA \#1 near } \\
\text { Homosassa }\end{array}$ & 284532082371001 & - & Citrus & 284533 & 823709 & $5 / 18 / 2010$ & 2 & \\
\hline
\end{tabular}


Table 1. Water-level measurements in Florida and parts of Georgia, South Carolina, and Alabama, May-June 2010.—Continued

[NGVD 29, National Geodetic Vertical Datum of 1929. Abbreviations for agency database: ACEPD, Alachua County Environmental Protection Department; FGS, Florida Geological Survey; GSA, Geological Survey of Alabama; NWFWMD, Northwest Florida Water Management District; SFWMD, South Florida Water Management District; SRWMD, Suwannee River Water Management District; USGSALWSC, U.S. Geological Survey Alabama Water Science Center; USGS-FLWSC, U.S. Geological Survey Florida Water Science Center; GAWSC, U.S. Geological Survey Georgia Water Science Center; SCWSC, U.S. Geological Survey South Carolina Water Science Center. Abbreviations for Station names: BLVD., boulevard; CO, county; CR, county road; E, east; HS, high school; IFAS, Institute of Food and Agricultural Services; LK, lake; MW, monitoring well; N, north; NE, northeast; NE, northeast; NF, national forest; NW, northwest; PK, park; R., river; RD., road; SE, southeast; SE, southeast; SR, state road; ST., street; TWR, tower; UFA, Upper Floridan aquifer; —, not available; \#, number; “, inches]

\begin{tabular}{|c|c|c|c|c|c|c|c|c|c|c|}
\hline State & $\begin{array}{c}\text { Agency } \\
\text { database }\end{array}$ & Station name & $\begin{array}{c}\text { USGS site } \\
\text { identification } \\
\text { number }\end{array}$ & $\begin{array}{l}\text { Local agency } \\
\text { unique site } \\
\text { identifier }\end{array}$ & County & $\begin{array}{l}\text { Latitude } \\
\text { (degree } \\
\text { minutes } \\
\text { and } \\
\text { seconds) } \\
\text { [DDMMSS] }\end{array}$ & $\begin{array}{l}\text { Longitude } \\
\text { (degree } \\
\text { minutes } \\
\text { and } \\
\text { seconds) } \\
\text { [DDMMSS] }\end{array}$ & $\begin{array}{c}\text { Date of } \\
\text { measure- } \\
\text { ment }\end{array}$ & $\begin{array}{c}\text { Water } \\
\text { level } \\
\text { altitude } \\
\text { (feet } \\
\text { above } \\
\text { NGVD 29) }\end{array}$ & Remarks \\
\hline FL & USGS-FLWSC & HOWARD BLVD. UFA WELL & 281443082055501 & - & Polk & 281444 & 820554 & $5 / 17 / 2010$ & 81 & \\
\hline FL & USGS-FLWSC & $\begin{array}{l}\text { INDIAN LK ESTATES GOLF } \\
\text { COURSE }\end{array}$ & 274746081202201 & - & Polk & 274747 & 812021 & $5 / 18 / 2010$ & 63 & \\
\hline FL & USGS-FLWSC & $\begin{array}{l}\text { INVERNESS VILLAGE EAST } \\
\text { WELL }\end{array}$ & 285037082213801 & - & Citrus & 285038 & 822137 & $5 / 19 / 2010$ & 20 & \\
\hline FL & USGS-FLWSC & $\begin{array}{l}\text { IR0040 RO MONITER-OSLO } \\
\text { RD }\end{array}$ & 273536080240201 & - & Indian River & 273537 & 802401 & $5 / 19 / 2010$ & 35 & \\
\hline FL & USGS-FLWSC & $\begin{array}{l}\text { IR0312 USDA-43R AVE SW } \\
\text { OF OSLO }\end{array}$ & 273435080255101 & - & Indian River & 273436 & 802550 & $5 / 19 / 2010$ & 31 & \\
\hline FL & USGS-FLWSC & $\begin{array}{l}\text { IR0366 MACE RANCH 4" } \\
\text { UFA }\end{array}$ & 274916080520701 & - & Indian River & 274917 & 805206 & $5 / 18 / 2010$ & 52 & \\
\hline FL & USGS-FLWSC & $\begin{array}{l}\text { IR0379 49TH ST N OF SR60 } \\
\text { W OF 66th AVE }\end{array}$ & 274055080281301 & - & Indian River & 274056 & 802812 & $5 / 19 / 2010$ & 32 & \\
\hline FL & USGS-FLWSC & IR0954 SJWCD UFA & 273515080344303 & - & Indian River & 273516 & 803442 & $5 / 19 / 2010$ & 40 & \\
\hline FL & USGS-FLWSC & $\begin{array}{l}\text { IR0955 DELTA FARMS OF3 } \\
\text { UFA }\end{array}$ & 273941080375401 & - & Indian River & 273943 & 803756 & $5 / 19 / 2010$ & 41 & \\
\hline FL & USGS-FLWSC & $\begin{array}{l}\text { IR0963 CORRIGAN RANCH } \\
\text { 6" UFA }\end{array}$ & 274126080304803 & - & Indian River & 274127 & 803048 & $5 / 19 / 2010$ & 36 & \\
\hline FL & USGS-FLWSC & $\begin{array}{l}\text { IR0968 Charles Hansen Lazy } \\
\text { H 6" UFA, SW shore Blue } \\
\text { Cypress Lake }\end{array}$ & 274217080464201 & - & Indian River & 274218 & 804641 & $5 / 19 / 2010$ & 42 & \\
\hline FL & USGS-FLWSC & IR1006 DODGER STADIUM & 273847080254703 & - & Indian River & 273848 & 802546 & $5 / 19 / 2010$ & 33 & \\
\hline FL & USGS-FLWSC & $\begin{array}{l}\text { IR1008 VERO BEACH } \\
\text { POWER 6" UFA }\end{array}$ & 273805080223802 & - & Indian River & 273803 & 802237 & $5 / 19 / 2010$ & 34 & \\
\hline FL & USGS-FLWSC & $\begin{array}{l}\text { IVEY'S NURSERY TURKEY } \\
\text { LK RD }\end{array}$ & 282923081282801 & - & Orange & 282924 & 812827 & $5 / 18 / 2010$ & 65 & \\
\hline
\end{tabular}


Table 1. Water-level measurements in Florida and parts of Georgia, South Carolina, and Alabama, May-June 2010.—Continued

[NGVD 29, National Geodetic Vertical Datum of 1929. Abbreviations for agency database: ACEPD, Alachua County Environmental Protection Department; FGS, Florida Geological Survey; GSA, Geological Survey of Alabama; NWFWMD, Northwest Florida Water Management District; SFWMD, South Florida Water Management District; SRWMD, Suwannee River Water Management District; USGSALWSC, U.S. Geological Survey Alabama Water Science Center; USGS-FLWSC, U.S. Geological Survey Florida Water Science Center; GAWSC, U.S. Geological Survey Georgia Water Science Center; SCWSC, U.S. Geological Survey South Carolina Water Science Center. Abbreviations for Station names: BLVD., boulevard; CO, county; CR, county road; E, east; HS, high school; IFAS, Institute of Food and Agricultural Services; LK, lake; MW, monitoring well; N, north; NE, northeast; NE, northeast; NF, national forest; NW, northwest; PK, park; R., river; RD., road; SE, southeast; SE, southeast; SR, state road; ST., street; TWR, tower; UFA, Upper Floridan aquifer; - - not available; \#, number; “, inches]

\begin{tabular}{|c|c|c|c|c|c|c|c|c|c|c|}
\hline State & $\begin{array}{l}\text { Agency } \\
\text { database }\end{array}$ & Station name & $\begin{array}{c}\text { USGS site } \\
\text { identification } \\
\text { number }\end{array}$ & $\begin{array}{l}\text { Local agency } \\
\text { unique site } \\
\text { identifier }\end{array}$ & County & $\begin{array}{l}\text { Latitude } \\
\text { (degree } \\
\text { minutes } \\
\text { and } \\
\text { seconds) } \\
\text { [DDMMS] }\end{array}$ & $\begin{array}{l}\text { Longitude } \\
\text { (degree } \\
\text { minutes } \\
\text { and } \\
\text { seconds) } \\
\text { [DDMMSS] }\end{array}$ & $\begin{array}{c}\text { Date of } \\
\text { measure- } \\
\text { ment }\end{array}$ & $\begin{array}{c}\text { Water } \\
\text { level } \\
\text { altitude } \\
\text { (feet } \\
\text { above } \\
\text { NGVD 29) }\end{array}$ & Remarks \\
\hline FL & USGS-FLWSC & $\begin{array}{l}\text { J.B. Sergeant 4" UFA near } \\
\text { Knights }\end{array}$ & 280455082021501 & - & Polk & 280456 & 820214 & $5 / 18 / 2010$ & 96 & \\
\hline FL & USGS-FLWSC & $\begin{array}{l}\text { J.O. Alston 2" UFA near Crystal } \\
\text { Springs }\end{array}$ & 281037082071801 & - & Pasco & 281038 & 820717 & $5 / 17 / 2010$ & 88 & \\
\hline FL & USGS-FLWSC & JC 27A & 284115082062601 & - & Sumter & 284116 & 820625 & $5 / 18 / 2010$ & 60 & \\
\hline FL & USGS-FLWSC & JC 32 & 284146082061401 & - & Sumter & 284147 & 820613 & $5 / 18 / 2010$ & 59 & \\
\hline FL & USGS-FLWSC & $\mathrm{JC} 42$ & 283952082022001 & - & Sumter & 283953 & 820219 & $5 / 18 / 2010$ & 80 & \\
\hline FL & USGS-FLWSC & JC 44 4" UFA NR BUSHNELL & 284119082034501 & - & Sumter & 284120 & 820344 & $5 / 18 / 2010$ & 78 & \\
\hline FL & USGS-FLWSC & $\mathrm{JC} 45$ & 284126082034501 & - & Sumter & 284127 & 820344 & $5 / 18 / 2010$ & 79 & \\
\hline FL & USGS-FLWSC & $\begin{array}{l}\text { JC } 47 \text { JUMPER CREEK WELL } \\
47 \text { NR NO }\end{array}$ & 283829082123701 & - & Sumter & 283830 & 821236 & $5 / 18 / 2010$ & 44 & \\
\hline FL & USGS-FLWSC & $\begin{array}{l}\text { JC } 56 \text { ROMP } 111 \text { UFA, } \\
\text { THOMPKINS PARK NR } \\
\text { COLEMAN }\end{array}$ & 284619082035101 & - & Sumter & 284620 & 820350 & $5 / 18 / 2010$ & 50 & \\
\hline FL & USGS-FLWSC & $\begin{array}{l}\text { JC } 57 \text { MASTERS AVE CTY } \\
\text { WELL AT WI }\end{array}$ & 285207082014501 & - & Sumter & 285208 & 820144 & $5 / 19 / 2010$ & 51 & \\
\hline FL & USGS-FLWSC & $\begin{array}{l}\text { JC } 58 \text { U S GEOLOGICAL } \\
\text { SURVEY CE } 3\end{array}$ & 285150082044001 & - & Sumter & 285151 & 820439 & $5 / 19 / 2010$ & 49 & \\
\hline FL & USGS-FLWSC & JC 65 & 283904082001601 & - & Sumter & 283905 & 820015 & $5 / 18 / 2010$ & 81 & \\
\hline FL & USGS-FLWSC & $\begin{array}{l}\text { JC } 71 \text { WOODWARD WELL } \\
\text { OFF OLD } 470\end{array}$ & 284449082055201 & - & Sumter & 284450 & 820551 & $5 / 18 / 2010$ & 42 & \\
\hline FL & USGS-FLWSC & $\begin{array}{l}\text { JC48B SEABOARD COAST } \\
\text { LINE RR USE }\end{array}$ & 283637082081501 & - & Sumter & 283638 & 820814 & $5 / 18 / 2010$ & 63 & \\
\hline FL & USGS-FLWSC & $\begin{array}{l}\text { Jct/o SR52/581 6" UFA near } \\
\text { Darby }\end{array}$ & 281926082212901 & - & Pasco & 281927 & 822128 & $5 / 17 / 2010$ & 69 & \\
\hline FL & USGS-FLWSC & $\begin{array}{l}\text { JIFFY (141620) CITY OF } \\
\text { GULF HAMMOCK 4" UFA }\end{array}$ & 291508082432901 & - & Levy & 291502 & 824329 & $5 / 26 / 2010$ & 11 & \\
\hline
\end{tabular}


Table 1. Water-level measurements in Florida and parts of Georgia, South Carolina, and Alabama, May-June 2010.—Continued

[NGVD 29, National Geodetic Vertical Datum of 1929. Abbreviations for agency database: ACEPD, Alachua County Environmental Protection Department; FGS, Florida Geological Survey; GSA, Geological Survey of Alabama; NWFWMD, Northwest Florida Water Management District; SFWMD, South Florida Water Management District; SRWMD, Suwannee River Water Management District; USGSALWSC, U.S. Geological Survey Alabama Water Science Center; USGS-FLWSC, U.S. Geological Survey Florida Water Science Center; GAWSC, U.S. Geological Survey Georgia Water Science Center; SCWSC, U.S. Geological Survey South Carolina Water Science Center. Abbreviations for Station names: BLVD., boulevard; CO, county; CR, county road; E, east; HS, high school; IFAS, Institute of Food and Agricultural Services; LK, lake; MW, monitoring well; N, north; NE, northeast; NE, northeast; NF, national forest; NW, northwest; PK, park; R., river; RD., road; SE, southeast; SE, southeast; SR, state road; ST., street; TWR, tower; UFA, Upper Floridan aquifer; —, not available; \#, number; “, inches]

\begin{tabular}{|c|c|c|c|c|c|c|c|c|c|c|}
\hline State & $\begin{array}{l}\text { Agency } \\
\text { database }\end{array}$ & Station name & $\begin{array}{c}\text { USGS site } \\
\text { identification } \\
\text { number }\end{array}$ & $\begin{array}{l}\text { Local agency } \\
\text { unique site } \\
\text { identifier }\end{array}$ & County & $\begin{array}{l}\text { Latitude } \\
\text { (degree } \\
\text { minutes } \\
\text { and } \\
\text { seconds) } \\
\text { [DDMMSS] }\end{array}$ & $\begin{array}{l}\text { Longitude } \\
\text { (degree } \\
\text { minutes } \\
\text { and } \\
\text { seconds) } \\
\text { ] [DDMMSS] }\end{array}$ & $\begin{array}{c}\text { Date of } \\
\text { measure- } \\
\text { ment }\end{array}$ & $\begin{array}{c}\text { Water } \\
\text { level } \\
\text { altitude } \\
\text { (feet } \\
\text { above } \\
\text { NGVD 29) }\end{array}$ & Remarks \\
\hline FL & USGS-FLWSC & $\begin{array}{l}\text { JOE OVERSTREET 4" UFA } \\
\text { NR LK KISSIMMEE }\end{array}$ & 275609081132001 & - & Osceola & 275610 & 811319 & $5 / 17 / 2010$ & 48 & \\
\hline FL & USGS-FLWSC & Jones 8" UFA near Arcadia & 271610081565401 & - & De Soto & 271613 & 815653 & $5 / 20 / 2010$ & 40 & \\
\hline FL & USGS-FLWSC & Justice 4" UFA near Hudson & 282238082362101 & - & Pasco & 282239 & 823620 & $5 / 19 / 2010$ & 25 & \\
\hline FL & USGS-FLWSC & $\begin{array}{l}\text { KATZ WELL AT LAKE } \\
\text { MIONA }\end{array}$ & 285422082001901 & - & Sumter & 285423 & 820018 & $5 / 19 / 2010$ & 48 & \\
\hline FL & USGS-FLWSC & $\begin{array}{l}\text { KEEN RANCH WELL } \\
\text { ASTATULA }\end{array}$ & 284241081402601 & - & Lake & 284242 & 814025 & $5 / 19 / 2010$ & 61 & \\
\hline FL & USGS-FLWSC & $\begin{array}{l}\text { KENT WELL AT LK PAN- } \\
\text { ASOFFKEE }\end{array}$ & 284809082080701 & - & Sumter & 284810 & 820806 & $5 / 19 / 2010$ & 39 & \\
\hline FL & USGS-FLWSC & $\begin{array}{l}\text { Kibler 8" UFA \#26B near } \\
\text { Bethany }\end{array}$ & 272838082142201 & - & Manatee & 272839 & 821421 & $5 / 17 / 2010$ & -7 & \\
\hline FL & USGS-FLWSC & $\begin{array}{l}\text { L. Knowles 4" UFA, } 30845 \text { CR } \\
\text { 435, Mt. Plymouth }\end{array}$ & 284757081320701 & - & Lake & 284758 & 813206 & $5 / 17 / 2010$ & 49 & \\
\hline FL & USGS-FLWSC & $\begin{array}{l}\text { L-0040 Alexander Springs 4" } \\
\text { UFA }\end{array}$ & 290647081342101 & - & Lake & 290648 & 813420 & $5 / 24 / 2010$ & 38 & \\
\hline FL & USGS-FLWSC & L-0043 LAKE YALE GROVES & 284445081462101 & - & Lake & 284446 & 814620 & $5 / 18 / 2010$ & 67 & \\
\hline FL & USGS-FLWSC & $\begin{array}{l}\text { L-0045 Astor (AG Edwards) } \\
\text { 6" UFA }\end{array}$ & 290950081315501 & - & Lake & 290951 & 813154 & $5 / 19 / 2010$ & 14 & \\
\hline FL & USGS-FLWSC & L-0051 SAND MINE UFA & 282241081443901 & - & Lake & 282242 & 814438 & $5 / 20 / 2010$ & 116 & \\
\hline FL & USGS-FLWSC & L-0052 JOHNS LK 4" UFA & 283128081404701 & - & Lake & 283129 & 814046 & $5 / 20 / 2010$ & 83 & \\
\hline FL & USGS-FLWSC & $\begin{array}{l}\text { L-0057 EVA 6" UFA AT } \\
\text { EVA;(DEEP) }\end{array}$ & 282245081492601 & - & Lake & 282246 & 814925 & $5 / 20 / 2010$ & 110 & \\
\hline FL & USGS-FLWSC & $\begin{array}{l}\text { L-0062 MASCOTTE UFA NR } \\
\text { MASCOTTE }\end{array}$ & 283204081544901 & - & Lake & 283205 & 815448 & $5 / 20 / 2010$ & 100 & \\
\hline FL & USGS-FLWSC & $\begin{array}{l}\text { L-0066 ALEXANDER SPGS } \\
\text { UFA NR ASTOR }\end{array}$ & 290451081344401 & - & Lake & 290452 & 813443 & $5 / 24 / 2010$ & 17 & \\
\hline
\end{tabular}


Table 1. Water-level measurements in Florida and parts of Georgia, South Carolina, and Alabama, May-June 2010.-Continued

[NGVD 29, National Geodetic Vertical Datum of 1929. Abbreviations for agency database: ACEPD, Alachua County Environmental Protection Department; FGS, Florida Geological Survey; GSA, Geological Survey of Alabama; NWFWMD, Northwest Florida Water Management District; SFWMD, South Florida Water Management District; SRWMD, Suwannee River Water Management District; USGSALWSC, U.S. Geological Survey Alabama Water Science Center; USGS-FLWSC, U.S. Geological Survey Florida Water Science Center; GAWSC, U.S. Geological Survey Georgia Water Science Center; SCWSC, U.S. Geological Survey South Carolina Water Science Center. Abbreviations for Station names: BLVD., boulevard; CO, county; CR, county road; E, east; HS, high school; IFAS, Institute of Food and Agricultural Services; LK, lake; MW, monitoring well; N, north; NE, northeast; NE, northeast; NF, national forest; NW, northwest; PK, park; R., river; RD., road; SE, southeast; SE, southeast; SR, state road; ST., street; TWR, tower; UFA, Upper Floridan aquifer; - - not available; \#, number; “, inches]

\begin{tabular}{|c|c|c|c|c|c|c|c|c|c|c|}
\hline State & $\begin{array}{c}\text { Agency } \\
\text { database }\end{array}$ & Station name & $\begin{array}{c}\text { USGS site } \\
\text { identification } \\
\text { number }\end{array}$ & $\begin{array}{c}\text { Local agency } \\
\text { unique site } \\
\text { identifier }\end{array}$ & County & $\begin{array}{l}\text { Latitude } \\
\text { (degree } \\
\text { minutes } \\
\text { and } \\
\text { seconds) } \\
\text { [DDMMS] }\end{array}$ & $\begin{array}{l}\text { Longitude } \\
\text { (degree } \\
\text { minutes } \\
\text { and } \\
\text { seconds) } \\
\text { [DDMMSS] }\end{array}$ & $\begin{array}{c}\text { Date of } \\
\text { measure- } \\
\text { ment }\end{array}$ & $\begin{array}{c}\text { Water } \\
\text { level } \\
\text { altitude } \\
\text { (feet } \\
\text { above } \\
\text { NGVD 29) }\end{array}$ & Remarks \\
\hline FL & USGS-FLWSC & $\begin{array}{l}\text { L-0095 GROVELAND TWR } \\
\text { UFA }\end{array}$ & 284122081534401 & - & Lake & 284123 & 815343 & $5 / 19 / 2010$ & 83 & \\
\hline FL & USGS-FLWSC & L-0199 TURNPIKE & 283355081411701 & - & Lake & 283356 & 814116 & $5 / 20 / 2010$ & 74 & \\
\hline FL & USGS-FLWSC & $\begin{array}{l}\text { L-0340 JUNIPER HUNT } \\
\text { CLUB UFA SUPPLY }\end{array}$ & 291448081381601 & - & Lake & 291449 & 813815 & $5 / 24 / 2010$ & 1 & \\
\hline FL & USGS-FLWSC & $\begin{array}{l}\text { L-0373 J. EICHELBURGER } \\
\text { UFA }\end{array}$ & 285257081434201 & - & Lake & 285258 & 814341 & $5 / 26 / 2010$ & 58 & \\
\hline FL & USGS-FLWSC & $\begin{array}{l}\text { L-0380 Austin Groves 8" UFA, } \\
\text { Umatilla }\end{array}$ & 285504081405901 & - & Lake & 285505 & 814058 & $5 / 26 / 2010$ & 53 & \\
\hline FL & USGS-FLWSC & $\begin{array}{l}\text { L-0390 PAUL SHOKLY UFA } \\
\text { 858-133-01 }\end{array}$ & 285827081331401 & - & Lake & 285828 & 813313 & $5 / 26 / 2010$ & 42 & \\
\hline FL & USGS-FLWSC & $\begin{array}{l}\text { L-0407 Camp Ocala 6" UFA, } \\
\text { Lake Sellars, OcalaNF }\end{array}$ & 290633081375201 & - & Lake & 290634 & 813751 & $5 / 24 / 2010$ & 42 & \\
\hline FL & USGS-FLWSC & $\begin{array}{l}\text { L-0441 USFS SOUTH OF } \\
\text { ASTOR }\end{array}$ & 290646081314001 & - & Lake & 290647 & 813139 & $5 / 24 / 2010$ & 18 & \\
\hline FL & USGS-FLWSC & L-0455 ASTOR 6" UFA & 291002081330601 & - & Lake & 291003 & 813305 & $5 / 24 / 2010$ & 17 & \\
\hline FL & USGS-FLWSC & $\begin{array}{l}\text { L-0555 GREEN SWAMP } \\
\text { TEST }\end{array}$ & 282318081544003 & - & Lake & 282319 & 815439 & $5 / 17 / 2010$ & 103 & \\
\hline FL & USGS-FLWSC & $\begin{array}{l}\text { L-0600 WOLF SINK 6" UFA } \\
\text { LCWA }\end{array}$ & 284725081361901 & - & Lake & 284726 & 813618 & $5 / 18 / 2010$ & 51 & \\
\hline FL & USGS-FLWSC & $\begin{array}{l}\text { L-0620 CABBAGE HAM- } \\
\text { MOCK UFA }\end{array}$ & 285357081472801 & - & Lake & 285358 & 814727 & $5 / 26 / 2010$ & 57 & \\
\hline FL & USGS-FLWSC & $\begin{array}{l}\text { L-0658 CITY OF MONT- } \\
\text { VERDE }\end{array}$ & 283608081403001 & - & Lake & 283609 & 814029 & $5 / 19 / 2010$ & 75 & \\
\hline FL & USGS-FLWSC & $\begin{array}{l}\text { L-0902 PALATLAKAHA } \\
\text { DAM PROPERTY }\end{array}$ & 284438081522301 & - & Lake & 284438 & 815223 & $5 / 19 / 2010$ & 71 & \\
\hline
\end{tabular}


Table 1. Water-level measurements in Florida and parts of Georgia, South Carolina, and Alabama, May-June 2010.—Continued

[NGVD 29, National Geodetic Vertical Datum of 1929. Abbreviations for agency database: ACEPD, Alachua County Environmental Protection Department; FGS, Florida Geological Survey; GSA, Geological Survey of Alabama; NWFWMD, Northwest Florida Water Management District; SFWMD, South Florida Water Management District; SRWMD, Suwannee River Water Management District; USGSALWSC, U.S. Geological Survey Alabama Water Science Center; USGS-FLWSC, U.S. Geological Survey Florida Water Science Center; GAWSC, U.S. Geological Survey Georgia Water Science Center; SCWSC, U.S. Geological Survey South Carolina Water Science Center. Abbreviations for Station names: BLVD., boulevard; CO, county; CR, county road; E, east; HS, high school; IFAS, Institute of Food and Agricultural Services; LK, lake; MW, monitoring well; N, north; NE, northeast; NE, northeast; NF, national forest; NW, northwest; PK, park; R., river; RD., road; SE, southeast; SE, southeast; SR, state road; ST., street; TWR, tower; UFA, Upper Floridan aquifer; - , not available; \#, number; “, inches]

\begin{tabular}{|c|c|c|c|c|c|c|c|c|c|c|}
\hline State & $\begin{array}{l}\text { Agency } \\
\text { database }\end{array}$ & Station name & $\begin{array}{c}\text { USGS site } \\
\text { identification } \\
\text { number }\end{array}$ & $\begin{array}{c}\text { Local agency } \\
\text { unique site } \\
\text { identifier }\end{array}$ & County & $\begin{array}{l}\text { Latitude } \\
\text { (degree } \\
\text { minutes } \\
\text { and } \\
\text { seconds) } \\
\text { [DDMMSS] }\end{array}$ & $\begin{array}{l}\text { Longitude } \\
\text { (degree } \\
\text { minutes } \\
\text { and } \\
\text { seconds) } \\
\text { [DDMMSS] }\end{array}$ & $\begin{array}{c}\text { Date of } \\
\text { measure- } \\
\text { ment }\end{array}$ & $\begin{array}{l}\text { Water } \\
\text { level } \\
\text { altitude } \\
\text { (feet } \\
\text { above } \\
\text { NGVD 29) }\end{array}$ & Remarks \\
\hline FL & USGS-FLWSC & L-1634 Golf Course Swnn Well & 262435081535101 & - & Lee & 262436 & 815350 & $5 / 17 / 2010$ & 38 & $\begin{array}{l}\text { Uncorrected hydrau- } \\
\text { lic head reported; } \\
\text { brackish to saline } \\
\text { water; not con- } \\
\text { toured on Potentio- } \\
\text { metric surface map }\end{array}$ \\
\hline FL & USGS-FLWSC & $\begin{array}{l}\text { L-5641 FIDDLESTICKS } \\
\text { SWNN WELL }\end{array}$ & 263115081483501 & - & Lee & 263116 & 814834 & $5 / 20 / 2010$ & 44 & $\begin{array}{l}\text { Uncorrected hydrau- } \\
\text { lic head reported; } \\
\text { brackish to saline } \\
\text { water; not con- } \\
\text { toured on Potentio- } \\
\text { metric surface map }\end{array}$ \\
\hline FL & USGS-FLWSC & $\begin{array}{l}\text { L-5708 (Green Groves) Swnn } \\
\text { Well Nr Alva }\end{array}$ & 264433081360601 & - & Lee & 264431 & 813609 & $5 / 19 / 2010$ & 53 & $\begin{array}{l}\text { Uncorrected hydrau- } \\
\text { lic head reported; } \\
\text { brackish to saline } \\
\text { water; not con- } \\
\text { toured on Potentio- } \\
\text { metric surface map }\end{array}$ \\
\hline FL & USGS-FLWSC & $\begin{array}{l}\text { LAKE ADAIR } 9 \text { DEEP WELL } \\
\text { AT ORLANDO }\end{array}$ & 283333081233501 & - & Orange & 283338 & 812334 & $5 / 17 / 2010$ & 47 & \\
\hline FL & USGS-FLWSC & $\begin{array}{l}\text { Lake Hatchineha Rd 8" UFA } \\
\text { near Lake Hamilton }\end{array}$ & 280229081325201 & - & Polk & 280230 & 813251 & $5 / 17 / 2010$ & 81 & \\
\hline FL & USGS-FLWSC & $\begin{array}{l}\text { Lake Placid Groves 12" UFA } \\
\text { near Lake Placid }\end{array}$ & 271223081202601 & - & Highlands & 271224 & 812025 & $5 / 19 / 2010$ & 49 & \\
\hline FL & USGS-FLWSC & $\begin{array}{l}\text { Lakeland Stadium 4" UFA, } \\
\text { Lakeland }\end{array}$ & 280420081570101 & - & Polk & 280421 & 815700 & $5 / 21 / 2010$ & 96 & \\
\hline FL & USGS-FLWSC & LAKEWOOD RETREAT & 282620082193801 & - & Hernando & 282621 & 821937 & $5 / 18 / 2010$ & 66 & \\
\hline FL & USGS-FLWSC & LAMMLEIN & 284519082150701 & - & Citrus & 284520 & 821506 & $5 / 19 / 2010$ & 40 & \\
\hline
\end{tabular}


Table 1. Water-level measurements in Florida and parts of Georgia, South Carolina, and Alabama, May-June 2010.-Continued

[NGVD 29, National Geodetic Vertical Datum of 1929. Abbreviations for agency database: ACEPD, Alachua County Environmental Protection Department; FGS, Florida Geological Survey; GSA, Geological Survey of Alabama; NWFWMD, Northwest Florida Water Management District; SFWMD, South Florida Water Management District; SRWMD, Suwannee River Water Management District; USGSALWSC, U.S. Geological Survey Alabama Water Science Center; USGS-FLWSC, U.S. Geological Survey Florida Water Science Center; GAWSC, U.S. Geological Survey Georgia Water Science Center; SCWSC, U.S. Geological Survey South Carolina Water Science Center. Abbreviations for Station names: BLVD., boulevard; CO, county; CR, county road; E, east; HS, high school; IFAS, Institute of Food and Agricultural Services; LK, lake; MW, monitoring well; N, north; NE, northeast; NE, northeast; NF, national forest; NW, northwest; PK, park; R., river; RD., road; SE, southeast; SE, southeast; SR, state road; ST., street; TWR, tower; UFA, Upper Floridan aquifer; - - not available; \#, number; “, inches]

\begin{tabular}{|c|c|c|c|c|c|c|c|c|c|c|}
\hline State & $\begin{array}{l}\text { Agency } \\
\text { database }\end{array}$ & Station name & $\begin{array}{c}\text { USGS site } \\
\text { identification } \\
\text { number }\end{array}$ & $\begin{array}{l}\text { Local agency } \\
\text { unique site } \\
\text { identifier }\end{array}$ & County & $\begin{array}{c}\text { Latitude } \\
\text { (degree } \\
\text { minutes } \\
\text { and } \\
\text { seconds) } \\
\text { [DDMMSS] }\end{array}$ & $\begin{array}{l}\text { Longitude } \\
\text { (degree } \\
\text { minutes } \\
\text { and } \\
\text { seconds) } \\
\text { ] [DDMMSS] }\end{array}$ & $\begin{array}{c}\text { Date of } \\
\text { measure- } \\
\text { ment }\end{array}$ & $\begin{array}{c}\text { Water } \\
\text { level } \\
\text { altitude } \\
\text { (feet } \\
\text { above } \\
\text { NGVD 29) }\end{array}$ & Remarks \\
\hline FL & USGS-FLWSC & $\begin{array}{l}\text { Lecanto 4" UFA \#1 near Chas- } \\
\text { sahowitzka }\end{array}$ & 284339082270401 & - & Citrus & 284340 & 822703 & $5 / 18 / 2010$ & 8 & \\
\hline FL & USGS-FLWSC & $\begin{array}{l}\text { Lecanto 4" UFA \#7 near } \\
\text { Lecanto }\end{array}$ & 285254082323001 & - & Citrus & 285255 & 823229 & $5 / 18 / 2010$ & 4 & \\
\hline FL & USGS-FLWSC & LEE WELL & 282428082134501 & - & Pasco & 282429 & 821344 & $5 / 17 / 2010$ & 64 & \\
\hline FL & USGS-FLWSC & Lithia Springs Nr Lithia & 02301600 & - & Hillsborough & 275158 & 821351 & $5 / 20 / 2010$ & 8 & \\
\hline FL & USGS-FLWSC & $\begin{array}{l}\text { LK ALFRED 12" UFA WELL } \\
\text { AT LK ALFRED }\end{array}$ & 280531081431601 & - & Polk & 280532 & 814315 & $5 / 17 / 2010$ & 120 & \\
\hline FL & USGS-FLWSC & $\begin{array}{l}\text { Lorena (Brant Lake) 4" UFA } \\
\text { near Lutz }\end{array}$ & 280738082282701 & - & Hillsborough & 280739 & 822826 & $5 / 18 / 2010$ & 53 & \\
\hline FL & USGS-FLWSC & LOUGHMAN UFA & 281532081345001 & - & Polk & 281533 & 813449 & $5 / 17 / 2010$ & 90 & \\
\hline FL & USGS-FLWSC & $\begin{array}{l}\text { Lower Hills Flood Detention } \\
\text { Area well } 22\end{array}$ & 280438082172901 & - & Hillsborough & 280439 & 821728 & $5 / 20 / 2010$ & 27 & \\
\hline FL & USGS-FLWSC & $\begin{array}{l}\text { LOWER WEKIVA RIVER } \\
\text { STATE PRESERVE 4" UFA } \\
\text { ARTESIAN }\end{array}$ & 285810081234101 & - & Lake & 285811 & 812340 & $5 / 26 / 2010$ & 27 & \\
\hline FL & USGS-FLWSC & $\begin{array}{l}\text { Lutz-Lake Fern 6" UFA near } \\
\text { Lutz }\end{array}$ & 280920082322101 & - & Hillsborough & 280921 & 823220 & $5 / 19 / 2010$ & 45 & \\
\hline FL & USGS-FLWSC & M. Murphy 4" UFA, Tampa & 275458082310301 & - & Hillsborough & 275459 & 823102 & $5 / 20 / 2010$ & 5 & \\
\hline FL & USGS-FLWSC & $\begin{array}{l}\text { M. Quagliani 6" UFA near } \\
\text { Plant City }\end{array}$ & 280413082061401 & - & Hillsborough & 280414 & 820613 & $5 / 17 / 2010$ & 89 & \\
\hline FL & USGS-FLWSC & M-0013 Moss Bluff 8" UFA & 290455081530401 & - & Marion & 290456 & 815303 & $5 / 25 / 2010$ & 53 & \\
\hline FL & USGS-FLWSC & $\begin{array}{l}\text { M-0021 LAKE GEORGE UFA } \\
\text { NR SALT SPRINGS }\end{array}$ & 291849081411401 & - & Marion & 291850 & 814113 & $5 / 26 / 2010$ & 16 & \\
\hline FL & USGS-FLWSC & $\begin{array}{l}\text { M-0024 USGS UFA CE } 84 \text { NR } \\
\text { SALT SPRINGS }\end{array}$ & 292200081510001 & - & Marion & 292201 & 815059 & $5 / 25 / 2010$ & 25 & \\
\hline
\end{tabular}


Table 1. Water-level measurements in Florida and parts of Georgia, South Carolina, and Alabama, May-June 2010.—Continued

[NGVD 29, National Geodetic Vertical Datum of 1929. Abbreviations for agency database: ACEPD, Alachua County Environmental Protection Department; FGS, Florida Geological Survey; GSA, Geological Survey of Alabama; NWFWMD, Northwest Florida Water Management District; SFWMD, South Florida Water Management District; SRWMD, Suwannee River Water Management District; USGSALWSC, U.S. Geological Survey Alabama Water Science Center; USGS-FLWSC, U.S. Geological Survey Florida Water Science Center; GAWSC, U.S. Geological Survey Georgia Water Science Center; SCWSC, U.S. Geological Survey South Carolina Water Science Center. Abbreviations for Station names: BLVD., boulevard; CO, county; CR, county road; E, east; HS, high school; IFAS, Institute of Food and Agricultural Services; LK, lake; MW, monitoring well; N, north; NE, northeast; NE, northeast; NF, national forest; NW, northwest; PK, park; R., river; RD., road; SE, southeast; SE, southeast; SR, state road; ST., street; TWR, tower; UFA, Upper Floridan aquifer; —, not available; \#, number; “, inches]

\begin{tabular}{|c|c|c|c|c|c|c|c|c|c|c|}
\hline State & $\begin{array}{l}\text { Agency } \\
\text { database }\end{array}$ & Station name & $\begin{array}{c}\text { USGS site } \\
\text { identification } \\
\text { number }\end{array}$ & $\begin{array}{l}\text { Local agency } \\
\text { unique site } \\
\text { identifier }\end{array}$ & County & $\begin{array}{l}\text { Latitude } \\
\text { (degree } \\
\text { minutes } \\
\text { and } \\
\text { seconds) } \\
\text { [DDMMSS] }\end{array}$ & $\begin{array}{l}\text { Longitude } \\
\text { (degree } \\
\text { minutes } \\
\text { and } \\
\text { seconds) } \\
\text { [DDMMSS] }\end{array}$ & $\begin{array}{c}\text { Date of } \\
\text { measure- } \\
\text { ment }\end{array}$ & $\begin{array}{c}\text { Water } \\
\text { level } \\
\text { altitude } \\
\text { (feet } \\
\text { above } \\
\text { NGVD 29) }\end{array}$ & Remarks \\
\hline $\mathrm{FL}$ & USGS-FLWSC & $\begin{array}{l}\text { M-0025 Gores Landing CE54 } \\
\text { 6" UFA near Ocala }\end{array}$ & 291740081562001 & - & Marion & 291741 & 815619 & $5 / 25 / 2010$ & 46 & \\
\hline FL & USGS-FLWSC & $\begin{array}{l}\text { M-0026 CE47 6" UFA near } \\
\text { Silver Springs }\end{array}$ & 291130082015001 & - & Marion & 291131 & 820149 & $5 / 25 / 2010$ & 43 & \\
\hline FL & USGS-FLWSC & $\begin{array}{l}\text { M-0028 (CE-76) 6" UFA, Hwy } \\
314 \text { near Ocala }\end{array}$ & 291100082010003 & - & Marion & 291101 & 820059 & $5 / 11 / 2010$ & 44 & \\
\hline FL & USGS-FLWSC & $\begin{array}{l}\text { M-0031 USGS OB WELL } \\
\text { CE36 AT PEDRO }\end{array}$ & 285900082072001 & - & Marion & 285901 & 820719 & $5 / 24 / 2010$ & 48 & \\
\hline FL & USGS-FLWSC & $\begin{array}{l}\text { M-0032 USGS UFA CE44 AT } \\
\text { OCALA }\end{array}$ & 291110082060001 & - & Marion & 291117 & 820605 & $5 / 25 / 2010$ & 43 & \\
\hline FL & USGS-FLWSC & $\begin{array}{l}\text { M-0037 USGS CE37 4" UFA } \\
\text { near Ocala }\end{array}$ & 290820082032001 & - & Marion & 290821 & 820319 & $5 / 25 / 2010$ & 45 & \\
\hline FL & USGS-FLWSC & $\begin{array}{l}\text { M-0041 CE33 4" UFA near } \\
\text { Ocala }\end{array}$ & 290400082091001 & - & Marion & 290401 & 820909 & $5 / 24 / 2010$ & 46 & \\
\hline FL & USGS-FLWSC & $\begin{array}{l}\text { M-0044 REDWATER LK UFA } \\
\text { NR LYNNE }\end{array}$ & 291117081540501 & - & Marion & 291118 & 815404 & $5 / 26 / 2010$ & 49 & \\
\hline $\mathrm{FL}$ & USGS-FLWSC & $\begin{array}{l}\text { M-0052 FORT MCCOY FIRE } \\
\text { TOWER UFA }\end{array}$ & 292204082022801 & - & Marion & 292206 & 820228 & $5 / 25 / 2010$ & 50 & \\
\hline FL & USGS-FLWSC & $\begin{array}{l}\text { M-0058 Wolf Sink CE81 4" } \\
\text { UFA near Santos }\end{array}$ & 290552082044701 & - & Marion & 290553 & 820446 & $5 / 26 / 2010$ & 46 & \\
\hline FL & USGS-FLWSC & $\begin{array}{l}\text { M-0059 ROMP } 120 \text { NR COT- } \\
\text { TON PLANT }\end{array}$ & 291059082190801 & - & Marion & 291060 & 821907 & $5 / 24 / 2010$ & 45 & \\
\hline $\mathrm{FL}$ & USGS-FLWSC & $\begin{array}{l}\text { M-0061 LOOKOUT TOWER } \\
\text { BOMBING RANGE UFA }\end{array}$ & 290628081425301 & - & Marion & 290629 & 814252 & $5 / 26 / 2010$ & 47 & \\
\hline $\mathrm{FL}$ & USGS-FLWSC & $\begin{array}{l}\text { M-0062 KOA UFA SR } 42 \mathrm{~W} \\
\text { OF ALTOONA }\end{array}$ & 285930081430901 & - & Marion & 285931 & 814308 & $5 / 26 / 2010$ & 52 & \\
\hline
\end{tabular}


Table 1. Water-level measurements in Florida and parts of Georgia, South Carolina, and Alabama, May-June 2010.-Continued

[NGVD 29, National Geodetic Vertical Datum of 1929. Abbreviations for agency database: ACEPD, Alachua County Environmental Protection Department; FGS, Florida Geological Survey; GSA, Geological Survey of Alabama; NWFWMD, Northwest Florida Water Management District; SFWMD, South Florida Water Management District; SRWMD, Suwannee River Water Management District; USGSALWSC, U.S. Geological Survey Alabama Water Science Center; USGS-FLWSC, U.S. Geological Survey Florida Water Science Center; GAWSC, U.S. Geological Survey Georgia Water Science Center; SCWSC, U.S. Geological Survey South Carolina Water Science Center. Abbreviations for Station names: BLVD., boulevard; CO, county; CR, county road; E, east; HS, high school; IFAS, Institute of Food and Agricultural Services; LK, lake; MW, monitoring well; N, north; NE, northeast; NE, northeast; NF, national forest; NW, northwest; PK, park; R., river; RD., road; SE, southeast; SE, southeast; SR, state road; ST., street; TWR, tower; UFA, Upper Floridan aquifer; - - not available; \#, number; “, inches]

\begin{tabular}{|c|c|c|c|c|c|c|c|c|c|c|}
\hline State & $\begin{array}{c}\text { Agency } \\
\text { database }\end{array}$ & Station name & $\begin{array}{c}\text { USGS site } \\
\text { identification } \\
\text { number }\end{array}$ & $\begin{array}{l}\text { Local agency } \\
\text { unique site } \\
\text { identifier }\end{array}$ & County & $\begin{array}{l}\text { Latitude } \\
\text { (degree } \\
\text { minutes } \\
\text { and } \\
\text { seconds) } \\
\text { [DDMMSS }\end{array}$ & $\begin{array}{l}\text { Longitude } \\
\text { (degree } \\
\text { minutes } \\
\text { and } \\
\text { seconds) } \\
\text { [DDMMSS] }\end{array}$ & $\begin{array}{c}\text { Date of } \\
\text { measure- } \\
\text { ment }\end{array}$ & $\begin{array}{c}\text { Water } \\
\text { level } \\
\text { altitude } \\
\text { (feet } \\
\text { above } \\
\text { NGVD 29) }\end{array}$ & Remarks \\
\hline FL & USGS-FLWSC & $\begin{array}{l}\text { M-0321 CE31 Golden Flake } \\
\text { UFA, Ocala }\end{array}$ & 291115082102901 & - & Marion & 291116 & 821028 & $5 / 24 / 2010$ & 45 & \\
\hline FL & USGS-FLWSC & M-0367 Huff UFA, McIntosh & 292622082131801 & - & Marion & 292623 & 821317 & $5 / 25 / 2010$ & 52 & \\
\hline FL & USGS-FLWSC & $\begin{array}{l}\text { M-0410 USGS 6" UFA, SW c/o } \\
\text { FSR88/31, OcalaNF }\end{array}$ & 292817081483602 & - & Marion & 292818 & 814835 & $5 / 25 / 2010$ & 20 & \\
\hline FL & USGS-FLWSC & $\begin{array}{l}\text { M-0419 MARION CTY SHER- } \\
\text { IFF UFA US } 301\end{array}$ & 291625082085901 & - & Marion & 291626 & 820858 & $5 / 25 / 2010$ & 44 & \\
\hline FL & USGS-FLWSC & $\begin{array}{l}\text { M-0441 G\&M RANCH NR } \\
\text { ORANGE SPRINGS }\end{array}$ & 292957081573002 & - & Marion & 292960 & 815730 & $5 / 25 / 2010$ & 54 & \\
\hline FL & USGS-FLWSC & $\begin{array}{l}\text { M-0443 CITRA RANCH NR } \\
\text { ISLAND GROVE }\end{array}$ & 292554082034501 & - & Marion & 292556 & 820346 & $5 / 25 / 2010$ & 54 & \\
\hline FL & USGS-FLWSC & $\begin{array}{l}\text { M-0463 Ft. McCoy Elementary } \\
\text { School 6" UFA }\end{array}$ & 292310081582201 & - & Marion & 292311 & 815821 & $5 / 25 / 2010$ & 52 & \\
\hline FL & USGS-FLWSC & $\begin{array}{l}\text { M-0465 Belleview Elementary } \\
\text { School 6" UFA }\end{array}$ & 290306082032101 & - & Marion & 290307 & 820322 & $5 / 24 / 2010$ & 48 & \\
\hline FL & USGS-FLWSC & $\begin{array}{l}\text { M-0467 Lake Weir Middle } \\
\text { School 6" UFA }\end{array}$ & 285953081590101 & - & Marion & 285954 & 815900 & $5 / 24 / 2010$ & 50 & \\
\hline FL & USGS-FLWSC & $\begin{array}{l}\text { M-0471 FOREST RD } 75 \\
\text { WELL }\end{array}$ & 292543081513301 & - & Marion & 292544 & 815122 & $5 / 25 / 2010$ & 21 & \\
\hline FL & USGS-FLWSC & $\begin{array}{l}\text { M-0528 ROTARY PARK } \\
\text { WELL }\end{array}$ & 290823082034301 & - & Marion & 290822 & 820343 & $5 / 25 / 2010$ & 45 & \\
\hline FL & USGS-FLWSC & $\begin{array}{l}\text { M-0627 INDIAN LAKE SF } \\
\text { 6-IN UFA WELL }\end{array}$ & 291653082035601 & - & Marion & 291653 & 820356 & $5 / 24 / 2010$ & 44 & \\
\hline FL & USGS-FLWSC & $\begin{array}{l}\text { M-0632 INDIAN LAKE SF } \\
\text { 3-IN UFA WELL }\end{array}$ & 291537082041001 & 一 & Marion & 291537 & 820410 & $5 / 24 / 2010$ & 44 & \\
\hline
\end{tabular}


Table 1. Water-level measurements in Florida and parts of Georgia, South Carolina, and Alabama, May-June 2010.—Continued

[NGVD 29, National Geodetic Vertical Datum of 1929. Abbreviations for agency database: ACEPD, Alachua County Environmental Protection Department; FGS, Florida Geological Survey; GSA, Geological Survey of Alabama; NWFWMD, Northwest Florida Water Management District; SFWMD, South Florida Water Management District; SRWMD, Suwannee River Water Management District; USGSALWSC, U.S. Geological Survey Alabama Water Science Center; USGS-FLWSC, U.S. Geological Survey Florida Water Science Center; GAWSC, U.S. Geological Survey Georgia Water Science Center; SCWSC, U.S. Geological Survey South Carolina Water Science Center. Abbreviations for Station names: BLVD., boulevard; CO, county; CR, county road; E, east; HS, high school; IFAS, Institute of Food and Agricultural Services; LK, lake; MW, monitoring well; N, north; NE, northeast; NE, northeast; NF, national forest; NW, northwest; PK, park; R., river; RD., road; SE, southeast; SE, southeast; SR, state road; ST., street; TWR, tower; UFA, Upper Floridan aquifer; —, not available; \#, number; “, inches]

\begin{tabular}{|c|c|c|c|c|c|c|c|c|c|c|}
\hline State & $\begin{array}{l}\text { Agency } \\
\text { database }\end{array}$ & Station name & $\begin{array}{l}\text { USGS site } \\
\text { identification } \\
\text { number }\end{array}$ & $\begin{array}{l}\text { Local agency } \\
\text { unique site } \\
\text { identifier }\end{array}$ & County & $\begin{array}{l}\text { Latitude } \\
\text { (degree } \\
\text { minutes } \\
\text { and } \\
\text { seconds) } \\
\text { [DDMMSS] }\end{array}$ & $\begin{array}{l}\text { Longitude } \\
\text { (degree } \\
\text { minutes } \\
\text { and } \\
\text { seconds) } \\
\text { [DDMMSS] }\end{array}$ & $\begin{array}{c}\text { Date of } \\
\text { measure- } \\
\text { ment }\end{array}$ & $\begin{array}{c}\text { Water } \\
\text { level } \\
\text { altitude } \\
\text { (feet } \\
\text { above } \\
\text { NGVD 29) }\end{array}$ & Remarks \\
\hline FL & USGS-FLWSC & $\begin{array}{l}\text { M-0637 SILVER RIVER } \\
\text { STATE PARK 6-IN UFA } \\
\text { WELL }\end{array}$ & 291248082022401 & - & Marion & 291248 & 820224 & $5 / 25 / 2010$ & 41 & \\
\hline FL & USGS-FLWSC & $\begin{array}{l}\text { M-0639 CITY OF OCALA 24- } \\
\text { IN SUPPLY WELL }\end{array}$ & 290854082071901 & - & Marion & 290854 & 820719 & $5 / 26 / 2010$ & 44 & \\
\hline FL & USGS-FLWSC & $\begin{array}{l}\text { M-0652 PERRY ACRES } \\
\text { SPRAYFIELD WELL } \\
\text { CW4D }\end{array}$ & 290450082021201 & - & Marion & 290450 & 820212 & $5 / 24 / 2010$ & 47 & \\
\hline FL & USGS-FLWSC & Mabry Calton CW-1 (3F) UFA & 270816082192601 & - & Sarasota & 270817 & 821925 & $5 / 18 / 2010$ & 29 & \\
\hline FL & USGS-FLWSC & $\begin{array}{l}\text { Mabry Carlton CW-2 (SM21A) } \\
\text { UFA }\end{array}$ & 270901082193102 & - & Sarasota & 270902 & 821930 & $5 / 18 / 2010$ & 27 & \\
\hline FL & USGS-FLWSC & Mabry Carlton CW-3 (6F) UFA & 271100082172701 & - & Sarasota & 271101 & 821726 & $5 / 18 / 2010$ & 27 & \\
\hline FL & USGS-FLWSC & $\begin{array}{l}\text { Mabry Carlton CW-6 (14-FS) } \\
\text { UFA }\end{array}$ & 270808082152601 & - & Sarasota & 270809 & 821525 & $5 / 18 / 2010$ & 32 & \\
\hline FL & USGS-FLWSC & $\begin{array}{l}\text { Mabry Carlton CW-7 (20F) } \\
\text { UFA }\end{array}$ & 271017082123101 & - & Sarasota & 271018 & 821230 & $5 / 18 / 2010$ & 34 & \\
\hline FL & USGS-FLWSC & $\begin{array}{l}\text { Mabry Carlton OM-41 SWNN } \\
\text { Well }\end{array}$ & 270928082172601 & - & Sarasota & 270929 & 821726 & $5 / 18 / 2010$ & 29 & \\
\hline FL & USGS-FLWSC & $\begin{array}{l}\text { Mabry Carlton UFA CW-5 } \\
\quad(5-14 \mathrm{FN})\end{array}$ & 270926082155101 & - & Sarasota & 270927 & 821550 & $5 / 18 / 2010$ & 31 & \\
\hline FL & USGS-FLWSC & $\begin{array}{l}\text { MACARTHUR TRAILER } \\
\text { PASTURE 8" UFA NR } \\
\text { BASINGER }\end{array}$ & 272354080524201 & - & Okeechobee & 272355 & 805241 & $5 / 18 / 2010$ & 42 & \\
\hline FL & USGS-FLWSC & MAHAFFEY UFA & 292718082202601 & - & Marion & 292719 & 822025 & $5 / 25 / 2010$ & 52 & \\
\hline FL & USGS-FLWSC & $\begin{array}{l}\text { Maranatha Village 6" UFA near } \\
\text { Sebring }\end{array}$ & 272835081251701 & - & Highlands & 273106 & 812517 & $5 / 19 / 2010$ & 78 & \\
\hline
\end{tabular}


Table 1. Water-level measurements in Florida and parts of Georgia, South Carolina, and Alabama, May-June 2010.-Continued

[NGVD 29, National Geodetic Vertical Datum of 1929. Abbreviations for agency database: ACEPD, Alachua County Environmental Protection Department; FGS, Florida Geological Survey; GSA, Geological Survey of Alabama; NWFWMD, Northwest Florida Water Management District; SFWMD, South Florida Water Management District; SRWMD, Suwannee River Water Management District; USGSALWSC, U.S. Geological Survey Alabama Water Science Center; USGS-FLWSC, U.S. Geological Survey Florida Water Science Center; GAWSC, U.S. Geological Survey Georgia Water Science Center; SCWSC, U.S. Geological Survey South Carolina Water Science Center. Abbreviations for Station names: BLVD., boulevard; CO, county; CR, county road; E, east; HS, high school; IFAS, Institute of Food and Agricultural Services; LK, lake; MW, monitoring well; N, north; NE, northeast; NE, northeast; NF, national forest; NW, northwest; PK, park; R., river; RD., road; SE, southeast; SE, southeast; SR, state road; ST., street; TWR, tower; UFA, Upper Floridan aquifer; - - not available; \#, number; “, inches]

\begin{tabular}{|c|c|c|c|c|c|c|c|c|c|c|}
\hline State & $\begin{array}{c}\text { Agency } \\
\text { database }\end{array}$ & Station name & $\begin{array}{c}\text { USGS site } \\
\text { identification } \\
\text { number }\end{array}$ & $\begin{array}{l}\text { Local agency } \\
\text { unique site } \\
\text { identifier }\end{array}$ & County & $\begin{array}{l}\text { Latitude } \\
\text { (degree } \\
\text { minutes } \\
\text { and } \\
\text { seconds) } \\
\text { [DDMMSS] }\end{array}$ & $\begin{array}{l}\text { Longitude } \\
\text { (degree } \\
\text { minutes } \\
\text { and } \\
\text { seconds) } \\
\text { ] [DDMMSS] }\end{array}$ & $\begin{array}{c}\text { Date of } \\
\text { measure- } \\
\text { ment }\end{array}$ & $\begin{array}{c}\text { Water } \\
\text { level } \\
\text { altitude } \\
\text { (feet } \\
\text { above } \\
\text { NGVD 29) }\end{array}$ & Remarks \\
\hline FL & USGS-FLWSC & $\begin{array}{l}\text { Marrls 10" UFA \#411 UFA near } \\
\text { Sweetwater }\end{array}$ & 272509081410401 & - & Hardee & 272510 & 814103 & $5 / 18 / 2010$ & 46 & \\
\hline FL & USGS-FLWSC & Marshall 6" UFA near Gardner & 272012081482501 & - & De Soto & 272013 & 814824 & $5 / 21 / 2010$ & 39 & \\
\hline FL & USGS-FLWSC & Masaryktown 6" UFA & 282540082275701 & - & Pasco & 282541 & 822756 & $5 / 17 / 2010$ & 25 & \\
\hline FL & USGS-FLWSC & $\begin{array}{l}\text { MCINTOSH ELLAP UFA } \\
\text { WELL }\end{array}$ & 280408082080801 & - & Hillsborough & 280409 & 820807 & $5 / 18 / 2010$ & 93 & \\
\hline FL & USGS-FLWSC & $\begin{array}{l}\text { McKay Creek Coastal Floridan } \\
\text { Well at Largo, FL }\end{array}$ & 275332082491201 & - & Pinellas & 275332 & 824912 & $5 / 17 / 2010$ & 6 & \\
\hline FL & USGS-FLWSC & $\begin{array}{l}\text { Meadowcroft 8" UFA near } \\
\text { Bradenton }\end{array}$ & 272855082362001 & - & Manatee & 272856 & 823619 & $5 / 20 / 2010$ & 12 & \\
\hline FL & USGS-FLWSC & $\begin{array}{l}\text { MERCANTILE LN (OSF-254) } \\
\text { NR POINCIANA }\end{array}$ & 281429081290501 & - & Osceola & 281430 & 812904 & $5 / 17 / 2010$ & 61 & \\
\hline FL & USGS-FLWSC & $\begin{array}{l}\text { MERRITT ISLAND INJEC- } \\
\text { TION WELL }\end{array}$ & 282524080422301 & - & Brevard & 282525 & 804222 & $5 / 25 / 2010$ & 19 & \\
\hline FL & USGS-FLWSC & MF-31 & 270847080103801 & - & Martin & 270848 & 801037 & $5 / 18 / 2010$ & 45 & $\begin{array}{l}\text { Uncorrected hydrau- } \\
\text { lic head reported; } \\
\text { brackish to saline } \\
\text { water; not con- } \\
\text { toured on Potentio- } \\
\text { metric surface map }\end{array}$ \\
\hline FL & USGS-FLWSC & $\begin{array}{l}\text { MF-3A INDIAN R PLANT } \\
\text { DEV CO }\end{array}$ & 271249080104403 & - & Martin & 271250 & 801043 & $5 / 18 / 2010$ & 50 & $\begin{array}{l}\text { Uncorrected hydrau- } \\
\text { lic head reported; } \\
\text { brackish to saline } \\
\text { water; not con- } \\
\text { toured on Potentio- } \\
\text { metric surface map }\end{array}$ \\
\hline
\end{tabular}


Table 1. Water-level measurements in Florida and parts of Georgia, South Carolina, and Alabama, May-June 2010.—Continued

[NGVD 29, National Geodetic Vertical Datum of 1929. Abbreviations for agency database: ACEPD, Alachua County Environmental Protection Department; FGS, Florida Geological Survey; GSA, Geological Survey of Alabama; NWFWMD, Northwest Florida Water Management District; SFWMD, South Florida Water Management District; SRWMD, Suwannee River Water Management District; USGSALWSC, U.S. Geological Survey Alabama Water Science Center; USGS-FLWSC, U.S. Geological Survey Florida Water Science Center; GAWSC, U.S. Geological Survey Georgia Water Science Center; SCWSC, U.S. Geological Survey South Carolina Water Science Center. Abbreviations for Station names: BLVD., boulevard; CO, county; CR, county road; E, east; HS, high school; IFAS, Institute of Food and Agricultural Services; LK, lake; MW, monitoring well; N, north; NE, northeast; NE, northeast; NF, national forest; NW, northwest; PK, park; R., river; RD., road; SE, southeast; SE, southeast; SR, state road; ST., street; TWR, tower; UFA, Upper Floridan aquifer; —, not available; \#, number; “, inches]

\begin{tabular}{|c|c|c|c|c|c|c|c|c|c|c|}
\hline State & $\begin{array}{l}\text { Agency } \\
\text { database }\end{array}$ & Station name & $\begin{array}{c}\text { USGS site } \\
\text { identification } \\
\text { number }\end{array}$ & $\begin{array}{l}\text { Local agency } \\
\text { unique site } \\
\text { identifier }\end{array}$ & County & $\begin{array}{l}\text { Latitude } \\
\text { (degree } \\
\text { minutes } \\
\text { and } \\
\text { seconds) } \\
\text { [DDMMSS] }\end{array}$ & $\begin{array}{l}\text { Longitude } \\
\text { (degree } \\
\text { minutes } \\
\text { and } \\
\text { seconds) } \\
\text { ] [DDMMSS] }\end{array}$ & $\begin{array}{c}\text { Date of } \\
\text { measure- } \\
\text { ment }\end{array}$ & $\begin{array}{c}\text { Water } \\
\text { level } \\
\text { altitude } \\
\text { (feet } \\
\text { above } \\
\text { NGVD 29) }\end{array}$ & Remarks \\
\hline FL & USGS-FLWSC & MF-9 ALLAPATAH PROP INC & 271003080280001 & - & Martin & 271004 & 802759 & $5 / 18 / 2010$ & 47 & $\begin{array}{l}\text { Uncorrected hydrau- } \\
\text { lic head reported; } \\
\text { brackish to saline } \\
\text { water; not con- } \\
\text { toured on Potentio- } \\
\text { metric surface map }\end{array}$ \\
\hline FL & USGS-FLWSC & $\begin{array}{l}\text { MILLER WELL AT KEN- } \\
\text { NETH CITY (4912 56TH } \\
\text { WAY) }\end{array}$ & 274904082423601 & - & Pinellas & 274905 & 824235 & $5 / 17 / 2010$ & 9 & \\
\hline FL & USGS-FLWSC & Mirror Lake UFA, St. Pete & 274624082383701 & - & Pinellas & 274625 & 823836 & $5 / 17 / 2010$ & 9 & \\
\hline FL & USGS-FLWSC & $\begin{array}{l}\text { Mission Hills 12" UFA near } \\
\text { Safety Harbor }\end{array}$ & 275842082430301 & - & Pinellas & 275843 & 824302 & $5 / 18 / 2010$ & 11 & \\
\hline FL & USGS-FLWSC & $\begin{array}{l}\text { Moon Lake 6" UFA near New } \\
\text { Port Richey }\end{array}$ & 281636082372001 & - & Pasco & 281637 & 823719 & $6 / 8 / 2010$ & 30 & \\
\hline FL & USGS-FLWSC & $\begin{array}{l}\text { Morris 4" UFA near Thonoto- } \\
\text { sassa }\end{array}$ & 280305082185101 & - & Hillsborough & 280306 & 821850 & $5 / 24 / 2010$ & 26 & \\
\hline FL & USGS-FLWSC & $\begin{array}{l}\text { Morris Bridge 14" UFA \#3A } \\
\text { near Branchton }\end{array}$ & 280655082193001 & - & Hillsborough & 280656 & 821929 & $5 / 24 / 2010$ & 32 & \\
\hline FL & USGS-FLWSC & $\begin{array}{l}\text { Morris Bridge 6" UFA \#10 near } \\
\text { Branchton }\end{array}$ & 280550082202901 & - & Hillsborough & 280551 & 822028 & $5 / 24 / 2010$ & 27 & \\
\hline FL & USGS-FLWSC & $\begin{array}{l}\text { Morris Bridge 6" UFA \#12 near } \\
\text { Branchton }\end{array}$ & 280605082184101 & - & Hillsborough & 280606 & 821840 & $5 / 24 / 2010$ & 28 & \\
\hline FL & USGS-FLWSC & $\begin{array}{l}\text { Morris Bridge 8" UFA \#13 near } \\
\text { Branchton }\end{array}$ & 280659082175201 & - & Hillsborough & 280656 & 821751 & $5 / 24 / 2010$ & 32 & \\
\hline FL & USGS-FLWSC & N FL Ave 18" UFA, Lakekand & 280338081572901 & - & Polk & 280339 & 815728 & $5 / 21 / 2010$ & 88 & \\
\hline FL & USGS-FLWSC & $\begin{array}{l}\text { N Lake Tarpon 8" UFA near } \\
\text { Tarpon Springs }\end{array}$ & 280852082414301 & - & Pinellas & 280853 & 824142 & $5 / 19 / 2010$ & 14 & \\
\hline FL & USGS-FLWSC & N Port 6" UFA near N Port & 270058082152502 & - & Sarasota & 270059 & 821524 & $5 / 20 / 2010$ & 27 & \\
\hline
\end{tabular}


Table 1. Water-level measurements in Florida and parts of Georgia, South Carolina, and Alabama, May-June 2010.-Continued

[NGVD 29, National Geodetic Vertical Datum of 1929. Abbreviations for agency database: ACEPD, Alachua County Environmental Protection Department; FGS, Florida Geological Survey; GSA, Geological Survey of Alabama; NWFWMD, Northwest Florida Water Management District; SFWMD, South Florida Water Management District; SRWMD, Suwannee River Water Management District; USGSALWSC, U.S. Geological Survey Alabama Water Science Center; USGS-FLWSC, U.S. Geological Survey Florida Water Science Center; GAWSC, U.S. Geological Survey Georgia Water Science Center; SCWSC, U.S. Geological Survey South Carolina Water Science Center. Abbreviations for Station names: BLVD., boulevard; CO, county; CR, county road; E, east; HS, high school; IFAS, Institute of Food and Agricultural Services; LK, lake; MW, monitoring well; N, north; NE, northeast; NE, northeast; NF, national forest; NW, northwest; PK, park; R., river; RD., road; SE, southeast; SE, southeast; SR, state road; ST., street; TWR, tower; UFA, Upper Floridan aquifer; - - not available; \#, number; “, inches]

\begin{tabular}{|c|c|c|c|c|c|c|c|c|c|c|}
\hline State & $\begin{array}{l}\text { Agency } \\
\text { database }\end{array}$ & Station name & $\begin{array}{c}\text { USGS site } \\
\text { identification } \\
\text { number }\end{array}$ & $\begin{array}{c}\text { Local agency } \\
\text { unique site } \\
\text { identifier }\end{array}$ & County & $\begin{array}{l}\text { Latitude } \\
\text { (degree } \\
\text { minutes } \\
\text { and } \\
\text { seconds) } \\
\text { [DDMMSS] }\end{array}$ & $\begin{array}{l}\text { Longitude } \\
\text { (degree } \\
\text { minutes } \\
\text { and } \\
\text { seconds) } \\
\text { [DDMMSS] }\end{array}$ & $\begin{array}{c}\text { Date of } \\
\text { measure- } \\
\text { ment }\end{array}$ & $\begin{array}{c}\text { Water } \\
\text { level } \\
\text { altitude } \\
\text { (feet } \\
\text { above } \\
\text { NGVD 29) }\end{array}$ & Remarks \\
\hline FL & USGS-FLWSC & $\begin{array}{l}\text { N-0019 Ft Clinch St Pk 5" } \\
\text { UFA, Fernandina Beach }\end{array}$ & 304213081270801 & - & Nassau & 304214 & 812707 & $5 / 17 / 2010$ & -7 & \\
\hline FL & USGS-FLWSC & $\begin{array}{c}\text { N-0121 ITT RAYONIER (OIL } \\
\text { TEST) UFA AT BECKER }\end{array}$ & 304005081380201 & - & Nassau & 304006 & 813801 & $5 / 17 / 2010$ & 35 & \\
\hline FL & USGS-FLWSC & $\begin{array}{l}\text { N-0130 UFA AT AMELIA } \\
\text { CITY }\end{array}$ & 303518081275002 & - & Nassau & 303519 & 812749 & $5 / 17 / 2010$ & 21 & \\
\hline FL & USGS-FLWSC & $\begin{array}{l}\text { N-0220 CALLAHAN FAIR- } \\
\text { GROUNDS 6" UFA }\end{array}$ & 303541081495001 & - & Nassau & 303544 & 814947 & $5 / 17 / 2010$ & 43 & \\
\hline FL & USGS-FLWSC & N-0221 ST MARY'S 6" UFA & 304658081571201 & - & Nassau & 304701 & 815709 & $5 / 17 / 2010$ & 42 & \\
\hline FL & USGS-FLWSC & $\begin{array}{l}\text { N-0237 CAREY ST FOREST } \\
\text { 6" UFA }\end{array}$ & 302409081551603 & - & Nassau & 302410 & 815515 & $5 / 17 / 2010$ & 41 & \\
\hline FL & USGS-FLWSC & $\begin{array}{l}\text { NCNB Nat'l Bank 4" UFA, } \\
\text { Tampa }\end{array}$ & 275630082275201 & - & Hillsborough & 275631 & 822751 & $5 / 20 / 2010$ & 8 & \\
\hline FL & USGS-FLWSC & Nininger 4" UFA \#857, Drexel & 281437082271401 & - & Pasco & 281438 & 822713 & $5 / 17 / 2010$ & 72 & \\
\hline FL & USGS-FLWSC & $\begin{array}{l}\text { Norris Cattle Co 12" UFA, } \\
\text { Homosassa Springs }\end{array}$ & 284803082351701 & - & Citrus & 284804 & 823516 & $5 / 18 / 2010$ & 2 & \\
\hline FL & USGS-FLWSC & $\begin{array}{l}\text { NORTH LECANTO DP WELL } \\
\text { NR LECANTO }\end{array}$ & 285414082284201 & - & Citrus & 285415 & 822841 & $5 / 20 / 2010$ & 4 & \\
\hline FL & USGS-FLWSC & $\begin{array}{l}\text { NW Pinellas Inj Mon 6" UFA } \\
\text { near Tarpon Springs }\end{array}$ & 280632082455001 & - & Pinellas & 280633 & 824549 & $5 / 19 / 2010$ & 3 & \\
\hline FL & USGS-FLWSC & Oakmont 8" UFA near Brandon & 275438082162301 & - & Hillsborough & 275439 & 821622 & $5 / 19 / 2010$ & 14 & \\
\hline FL & USGS-FLWSC & $\begin{array}{l}\text { OBSERVATION WELL CE74 } \\
\text { NR OCALA }\end{array}$ & 290215082152401 & - & Marion & 290216 & 821523 & $5 / 24 / 2010$ & 45 & \\
\hline FL & USGS-FLWSC & $\begin{array}{l}\text { OK0001 8" UFA NR FT } \\
\text { DRUM TD STEEL }\end{array}$ & 273127080481401 & - & Okeechobee & 273128 & 804813 & $5 / 19 / 2010$ & 44 & \\
\hline FL & USGS-FLWSC & $\begin{array}{l}\text { OKAHUMPKA WORSHIP } \\
\text { CENTER }\end{array}$ & 284528081530201 & - & Lake & 284529 & 815301 & $5 / 18 / 2010$ & 67 & \\
\hline
\end{tabular}


Table 1. Water-level measurements in Florida and parts of Georgia, South Carolina, and Alabama, May-June 2010.—Continued

[NGVD 29, National Geodetic Vertical Datum of 1929. Abbreviations for agency database: ACEPD, Alachua County Environmental Protection Department; FGS, Florida Geological Survey; GSA, Geological Survey of Alabama; NWFWMD, Northwest Florida Water Management District; SFWMD, South Florida Water Management District; SRWMD, Suwannee River Water Management District; USGSALWSC, U.S. Geological Survey Alabama Water Science Center; USGS-FLWSC, U.S. Geological Survey Florida Water Science Center; GAWSC, U.S. Geological Survey Georgia Water Science Center; SCWSC, U.S. Geological Survey South Carolina Water Science Center. Abbreviations for Station names: BLVD., boulevard; CO, county; CR, county road; E, east; HS, high school; IFAS, Institute of Food and Agricultural Services; LK, lake; MW, monitoring well; N, north; NE, northeast; NE, northeast; NF, national forest; NW, northwest; PK, park; R., river; RD., road; SE, southeast; SE, southeast; SR, state road; ST., street; TWR, tower; UFA, Upper Floridan aquifer; —, not available; \#, number; “, inches]

\begin{tabular}{|c|c|c|c|c|c|c|c|c|c|c|}
\hline State & $\begin{array}{l}\text { Agency } \\
\text { database }\end{array}$ & Station name & $\begin{array}{c}\text { USGS site } \\
\text { identification } \\
\text { number }\end{array}$ & $\begin{array}{l}\text { Local agency } \\
\text { unique site } \\
\text { identifier }\end{array}$ & County & $\begin{array}{l}\text { Latitude } \\
\text { (degree } \\
\text { minutes } \\
\text { and } \\
\text { seconds) } \\
\text { [DDMMSS] }\end{array}$ & $\begin{array}{l}\text { Longitude } \\
\text { (degree } \\
\text { minutes } \\
\text { and } \\
\text { seconds) } \\
\text { [DDMMSS] }\end{array}$ & $\begin{array}{c}\text { Date of } \\
\text { measure- } \\
\text { ment }\end{array}$ & $\begin{array}{c}\text { Water } \\
\text { level } \\
\text { altitude } \\
\text { (feet } \\
\text { above } \\
\text { NGVD 29) }\end{array}$ & Remarks \\
\hline FL & USGS-FLWSC & $\begin{array}{l}\text { OKF-18 BASS W. N OF BAS- } \\
\text { INGER }\end{array}$ & 272726081003901 & - & Okeechobee & 272727 & 810038 & $5 / 17 / 2010$ & 46 & \\
\hline FL & USGS-FLWSC & OKF-23 OKEЕCHOBEE & 271514080511601 & - & Okeechobee & 271515 & 805115 & $5 / 18 / 2010$ & 47 & \\
\hline FL & USGS-FLWSC & OKF-31 OKEЕCHOBEE & 271340080504001 & - & Okeechobee & 271341 & 805039 & $5 / 18 / 2010$ & 47 & \\
\hline FL & USGS-FLWSC & $\begin{array}{l}\text { OKF-56 4.35MI OFF US98 } \\
\text { MICCO RD }\end{array}$ & 272704081053501 & - & Okeechobee & 272705 & 810534 & $5 / 17 / 2010$ & 48 & \\
\hline FL & USGS-FLWSC & OR0007 BITHLO 1 16" UFA & 283249081053201 & - & Orange & 283250 & 810531 & $5 / 17 / 2010$ & 35 & \\
\hline FL & USGS-FLWSC & $\begin{array}{l}\text { OR0029 PALMETTO 3" UFA } \\
\text { NR BITHLO }\end{array}$ & 282348080564701 & - & Orange & 282349 & 805646 & $5 / 17 / 2010$ & 35 & \\
\hline FL & USGS-FLWSC & $\begin{array}{l}\text { OR0030 USGS BOGGY CK } \\
\text { RD UFA @ CO LINE NR } \\
\text { TAFT }\end{array}$ & 282051081183401 & - & Orange & 282052 & 811833 & $5 / 17 / 2010$ & 46 & \\
\hline FL & USGS-FLWSC & $\begin{array}{l}\text { OR0046 Lake Adair } 104 " \\
\text { UFA, Orlando }\end{array}$ & 283333081233502 & - & Orange & 283327 & 812335 & $5 / 17 / 2010$ & 48 & \\
\hline FL & USGS-FLWSC & OR0047 6" UFA, Orla Vista & 283253081283401 & - & Orange & 283254 & 812833 & $5 / 18 / 2010$ & 61 & \\
\hline FL & USGS-FLWSC & $\begin{array}{l}\text { OR0064 LK OLIVER 6" UFA } \\
\text { NR LK OLIVER }\end{array}$ & 282202081384601 & - & Orange & 282203 & 813845 & $5 / 18 / 2010$ & 109 & \\
\hline FL & USGS-FLWSC & OR0082 COCOA D 4" UFA & 282531081095701 & - & Orange & 282532 & 810956 & $5 / 19 / 2010$ & 34 & \\
\hline FL & USGS-FLWSC & $\begin{array}{l}\text { OR0106 PLYMOUTH TOWER } \\
\text { UFA }\end{array}$ & 284230081345301 & - & Orange & 284231 & 813452 & $5 / 18 / 2010$ & 55 & \\
\hline FL & USGS-FLWSC & $\begin{array}{l}\text { OR0265 COCOA F NR } \\
\text { BITHLO }\end{array}$ & 282739081054501 & - & Orange & 282740 & 810543 & $5 / 17 / 2010$ & 35 & \\
\hline FL & USGS-FLWSC & OR0468 LK IVANHOE UFA & 283340081222803 & - & Orange & 283341 & 812227 & $5 / 18 / 2010$ & 48 & \\
\hline FL & USGS-FLWSC & $\begin{array}{l}\text { OR0548 WEKIWA SP ST PK } \\
\text { UFA NR FOREST CITY }\end{array}$ & 284238081275803 & - & Orange & 284239 & 812757 & $5 / 18 / 2010$ & 21 & \\
\hline FL & USGS-FLWSC & OR0669 COCOA 13T 10" UFA & 282339081010001 & - & Orange & 282340 & 810058 & $5 / 17 / 2010$ & 36 & \\
\hline FL & USGS-FLWSC & OR0678 ALAFAYA TRAIL & 283007081122705 & - & Orange & 283008 & 811226 & $5 / 17 / 2010$ & 39 & \\
\hline
\end{tabular}


Table 1. Water-level measurements in Florida and parts of Georgia, South Carolina, and Alabama, May-June 2010.-Continued

[NGVD 29, National Geodetic Vertical Datum of 1929. Abbreviations for agency database: ACEPD, Alachua County Environmental Protection Department; FGS, Florida Geological Survey; GSA, Geological Survey of Alabama; NWFWMD, Northwest Florida Water Management District; SFWMD, South Florida Water Management District; SRWMD, Suwannee River Water Management District; USGSALWSC, U.S. Geological Survey Alabama Water Science Center; USGS-FLWSC, U.S. Geological Survey Florida Water Science Center; GAWSC, U.S. Geological Survey Georgia Water Science Center; SCWSC, U.S. Geological Survey South Carolina Water Science Center. Abbreviations for Station names: BLVD., boulevard; CO, county; CR, county road; E, east; HS, high school; IFAS, Institute of Food and Agricultural Services; LK, lake; MW, monitoring well; N, north; NE, northeast; NE, northeast; NF, national forest; NW, northwest; PK, park; R., river; RD., road; SE, southeast; SE, southeast; SR, state road; ST., street; TWR, tower; UFA, Upper Floridan aquifer; - - not available; \#, number; “, inches]

\begin{tabular}{|c|c|c|c|c|c|c|c|c|c|c|}
\hline State & $\begin{array}{c}\text { Agency } \\
\text { database }\end{array}$ & Station name & $\begin{array}{c}\text { USGS site } \\
\text { identification } \\
\text { number }\end{array}$ & $\begin{array}{l}\text { Local agency } \\
\text { unique site } \\
\text { identifier }\end{array}$ & County & $\begin{array}{l}\text { Latitude } \\
\text { (degree } \\
\text { minutes } \\
\text { and } \\
\text { seconds) } \\
\text { [DDMMSS] }\end{array}$ & $\begin{array}{l}\text { Longitude } \\
\text { (degree } \\
\text { minutes } \\
\text { and } \\
\text { seconds) } \\
\text { [DDMMSS] }\end{array}$ & $\begin{array}{c}\text { Date of } \\
\text { measure- } \\
\text { ment }\end{array}$ & $\begin{array}{c}\text { Water } \\
\text { level } \\
\text { altitude } \\
\text { (feet } \\
\text { above } \\
\text { NGVD 29) }\end{array}$ & Remarks \\
\hline FL & USGS-FLWSC & $\begin{array}{l}\text { OR-0796 APOPKA CRATE } \\
\text { MILL }\end{array}$ & 283959081303101 & - & Orange & 283960 & 813030 & $5 / 18 / 2010$ & 50 & \\
\hline FL & USGS-FLWSC & Orleans St 20" UFA, Lakeland & 280053081572301 & - & Polk & 280054 & 815722 & $5 / 21 / 2010$ & 78 & \\
\hline FL & USGS-FLWSC & $\begin{array}{l}\text { OS0001 LAKE JOEL 8" UFA } \\
\text { NR ASHTON }\end{array}$ & 281714081093001 & - & Osceola & 281715 & 810929 & $5 / 18 / 2010$ & 43 & \\
\hline FL & USGS-FLWSC & $\begin{array}{l}\text { OS0033 DSR-38 LAKE POIN- } \\
\text { SETT }\end{array}$ & 281632080515001 & - & Osceola & 281633 & 805149 & $5 / 18 / 2010$ & 38 & \\
\hline FL & USGS-FLWSC & $\begin{array}{l}\text { OS0047 TH-10 4" UFA WIL- } \\
\text { LIAMS RD }\end{array}$ & 275852081030501 & - & Osceola & 275853 & 810304 & $5 / 17 / 2010$ & 43 & \\
\hline FL & USGS-FLWSC & $\begin{array}{l}\text { OS0231 CAMPBELL RANCH } \\
\text { 6" UFA }\end{array}$ & 274944080573302 & - & Osceola & 274946 & 805732 & $5 / 18 / 2010$ & 44 & \\
\hline FL & USGS-FLWSC & $\begin{array}{l}\text { OS-0238 TH-4 DEER PK NW } \\
\text { UFA NR HOLOPAW }\end{array}$ & 281354080563301 & - & Osceola & 281355 & 805632 & $5 / 18 / 2010$ & 40 & \\
\hline FL & USGS-FLWSC & $\begin{array}{l}\text { OSF-34 KISS PK OFF } \\
\text { WHALEY RD }\end{array}$ & 281146081211701 & - & Osceola & 281147 & 812116 & $5 / 17 / 2010$ & 49 & \\
\hline FL & USGS-FLWSC & $\begin{array}{l}\text { OSF-42 (OS0038) 4.4MI W } \\
\text { YEEHAW JUN SR60 }\end{array}$ & 274307080582401 & - & Osceola & 274308 & 805823 & $5 / 19 / 2010$ & 44 & \\
\hline FL & USGS-FLWSC & $\begin{array}{l}\text { OSF-44 ST CLOUD POWER } \\
\text { PLANT }\end{array}$ & 281456081171701 & - & Osceola & 281457 & 811716 & $5 / 17 / 2010$ & 47 & \\
\hline FL & USGS-FLWSC & $\begin{array}{l}\text { OSF-53 S-61 WELL NR AL- } \\
\text { COMA }\end{array}$ & 280823081210301 & - & Osceola & 280824 & 812102 & $5 / 17 / 2010$ & 52 & \\
\hline FL & USGS-FLWSC & $\begin{array}{l}\text { Ozello 4" UFA \#3 near Crystal } \\
\text { River }\end{array}$ & 285020082365301 & - & Citrus & 285021 & 823652 & $5 / 18 / 2010$ & 1 & \\
\hline FL & USGS-FLWSC & $\begin{array}{l}\text { Ozello 4" UFA \#4 near Crystal } \\
\text { River }\end{array}$ & 285102082361001 & - & Citrus & 285103 & 823609 & $5 / 18 / 2010$ & 2 & \\
\hline FL & USGS-FLWSC & P-0076 A J ROBERTS UFA & 294255081323501 & - & Putnam & 294256 & 813234 & $5 / 26 / 2010$ & 19 & \\
\hline FL & USGS-FLWSC & P-0123 DHQ UFA & 293951081413901 & - & Putnam & 293952 & 814138 & $5 / 25 / 2010$ & 29 & \\
\hline
\end{tabular}


Table 1. Water-level measurements in Florida and parts of Georgia, South Carolina, and Alabama, May-June 2010.—Continued

[NGVD 29, National Geodetic Vertical Datum of 1929. Abbreviations for agency database: ACEPD, Alachua County Environmental Protection Department; FGS, Florida Geological Survey; GSA, Geological Survey of Alabama; NWFWMD, Northwest Florida Water Management District; SFWMD, South Florida Water Management District; SRWMD, Suwannee River Water Management District; USGSALWSC, U.S. Geological Survey Alabama Water Science Center; USGS-FLWSC, U.S. Geological Survey Florida Water Science Center; GAWSC, U.S. Geological Survey Georgia Water Science Center; SCWSC, U.S. Geological Survey South Carolina Water Science Center. Abbreviations for Station names: BLVD., boulevard; CO, county; CR, county road; E, east; HS, high school; IFAS, Institute of Food and Agricultural Services; LK, lake; MW, monitoring well; N, north; NE, northeast; NE, northeast; NF, national forest; NW, northwest; PK, park; R., river; RD., road; SE, southeast; SE, southeast; SR, state road; ST., street; TWR, tower; UFA, Upper Floridan aquifer; —, not available; \#, number; “, inches]

\begin{tabular}{|c|c|c|c|c|c|c|c|c|c|c|}
\hline State & $\begin{array}{c}\text { Agency } \\
\text { database }\end{array}$ & Station name & $\begin{array}{c}\text { USGS site } \\
\text { identification } \\
\text { number }\end{array}$ & $\begin{array}{l}\text { Local agency } \\
\text { unique site } \\
\text { identifier }\end{array}$ & County & $\begin{array}{l}\text { Latitude } \\
\text { (degree } \\
\text { minutes } \\
\text { and } \\
\text { seconds) } \\
\text { [DDMMSS] }\end{array}$ & $\begin{array}{l}\text { Longitude } \\
\text { (degree } \\
\text { minutes } \\
\text { and } \\
\text { seconds) } \\
\text { [DDMMSS] }\end{array}$ & $\begin{array}{c}\text { Date of } \\
\text { measure- } \\
\text { ment }\end{array}$ & $\begin{array}{c}\text { Water } \\
\text { level } \\
\text { altitude } \\
\text { (feet } \\
\text { above } \\
\text { NGVD 29) }\end{array}$ & Remarks \\
\hline FL & USGS-FLWSC & $\begin{array}{l}\text { P-0132 GREENWAY NR } \\
\text { RODMAN }\end{array}$ & 293228081495301 & - & Putnam & 293229 & 814952 & $5 / 25 / 2010$ & 31 & \\
\hline FL & USGS-FLWSC & $\begin{array}{l}\text { P-0166 CAMP KATERI WELL } \\
\text { NR ORANGE SPRINGS, FL }\end{array}$ & 293103081575501 & - & Putnam & 293104 & 815754 & $5 / 25 / 2010$ & 61 & \\
\hline FL & USGS-FLWSC & $\begin{array}{l}\text { P-0172 } 93913411 \text { 10S27E04 } \\
\text { PUTNAM } 29 \text { UFA }\end{array}$ & 293933081342801 & - & Putnam & 293934 & 813429 & $5 / 26 / 2010$ & 17 & \\
\hline FL & USGS-FLWSC & $\begin{array}{l}\text { P-0246 THUNDERBIRD AIR- } \\
\text { PARK (COL SAULS) }\end{array}$ & 292824081341501 & - & Putnam & 292825 & 813414 & $5 / 25 / 2010$ & 31 & \\
\hline FL & USGS-FLWSC & $\begin{array}{l}\text { P-0270 FRUITLAND } \\
\text { HANDYWAY 6" UFA }\end{array}$ & 292528081383501 & - & Putnam & 292538 & 813835 & $5 / 25 / 2010$ & 17 & \\
\hline FL & USGS-FLWSC & $\begin{array}{l}\text { P-0306 } 933152 \text { CE60 8" UFA } \\
\text { ARTESIAN }\end{array}$ & 293300081523901 & - & Putnam & 293301 & 815238 & $5 / 25 / 2010$ & 60 & \\
\hline FL & USGS-FLWSC & $\begin{array}{l}\text { P-0382 GEORGE MAIN } \\
\text { FERNERY 6" UFA }\end{array}$ & 293113081370301 & - & Putnam & 293114 & 813702 & $5 / 25 / 2010$ & 28 & \\
\hline FL & USGS-FLWSC & $\begin{array}{l}\text { P-0396 Welaka Fish Hatchery } \\
\text { 6" UFA Artesian, Fruitland }\end{array}$ & 292628081385501 & - & Putnam & 292629 & 813854 & $5 / 25 / 2010$ & 12 & \\
\hline FL & USGS-FLWSC & P-0410 Union Camp 4" UFA \#9 & 292218081333101 & - & Putnam & 292219 & 813330 & $5 / 25 / 2010$ & 27 & \\
\hline FL & USGS-FLWSC & $\begin{array}{l}\text { P-0421 DRAYTON ISLAND } \\
\text { 4" UFA ARTESIAN }\end{array}$ & 292254081382101 & - & Putnam & 292255 & 813820 & $5 / 25 / 2010$ & 11 & \\
\hline FL & USGS-FLWSC & $\begin{array}{l}\text { P-0427 UFA NR FRONTIER D } \\
\text { H NR SALT SPRINGS }\end{array}$ & 292435081441301 & - & Putnam & 292436 & 814412 & $5 / 25 / 2010$ & 9 & \\
\hline FL & USGS-FLWSC & $\begin{array}{l}\text { P-0464 COWPEN LK UFA } \\
\text { DRAINAGE }\end{array}$ & 293633081594601 & - & Putnam & 293634 & 815945 & $5 / 25 / 2010$ & 77 & \\
\hline FL & USGS-FLWSC & $\begin{array}{l}\text { P-0472 JOHNSONS FIELD } \\
\text { UFA NR WELAKA }\end{array}$ & 292824081443301 & - & Putnam & 292825 & 814432 & $5 / 25 / 2010$ & 8 & \\
\hline FL & USGS-FLWSC & $\begin{array}{l}\text { P-0474 SAN MATEO TOWER } \\
\text { UFA }\end{array}$ & 293554081342601 & - & Putnam & 293555 & 813425 & $5 / 25 / 2010$ & 17 & \\
\hline
\end{tabular}


Table 1. Water-level measurements in Florida and parts of Georgia, South Carolina, and Alabama, May-June 2010.-Continued

[NGVD 29, National Geodetic Vertical Datum of 1929. Abbreviations for agency database: ACEPD, Alachua County Environmental Protection Department; FGS, Florida Geological Survey; GSA, Geological Survey of Alabama; NWFWMD, Northwest Florida Water Management District; SFWMD, South Florida Water Management District; SRWMD, Suwannee River Water Management District; USGSALWSC, U.S. Geological Survey Alabama Water Science Center; USGS-FLWSC, U.S. Geological Survey Florida Water Science Center; GAWSC, U.S. Geological Survey Georgia Water Science Center; SCWSC, U.S. Geological Survey South Carolina Water Science Center. Abbreviations for Station names: BLVD., boulevard; CO, county; CR, county road; E, east; HS, high school; IFAS, Institute of Food and Agricultural Services; LK, lake; MW, monitoring well; N, north; NE, northeast; NE, northeast; NF, national forest; NW, northwest; PK, park; R., river; RD., road; SE, southeast; SE, southeast; SR, state road; ST., street; TWR, tower; UFA, Upper Floridan aquifer; - - not available; \#, number; “, inches]

\begin{tabular}{|c|c|c|c|c|c|c|c|c|c|c|}
\hline State & $\begin{array}{c}\text { Agency } \\
\text { database }\end{array}$ & Station name & $\begin{array}{c}\text { USGS site } \\
\text { identification } \\
\text { number }\end{array}$ & $\begin{array}{l}\text { Local agency } \\
\text { unique site } \\
\text { identifier }\end{array}$ & County & $\begin{array}{c}\text { Latitude } \\
\text { (degree } \\
\text { minutes } \\
\text { and } \\
\text { seconds) } \\
\text { [DDMMSS] }\end{array}$ & $\begin{array}{l}\text { Longitude } \\
\text { (degree } \\
\text { minutes } \\
\text { and } \\
\text { seconds) } \\
\text { [DDMMSS] }\end{array}$ & $\begin{array}{c}\text { Date of } \\
\text { measure- } \\
\text { ment }\end{array}$ & $\begin{array}{l}\text { Water } \\
\text { level } \\
\text { altitude } \\
\text { (feet } \\
\text { above } \\
\text { NGVD 29) }\end{array}$ & Remarks \\
\hline FL & USGS-FLWSC & $\begin{array}{l}\text { P-0510 HOLLISTER WORKC- } \\
\text { TR UFA }\end{array}$ & 293733081474801 & - & Putnam & 293734 & 814747 & $5 / 25 / 2010$ & 49 & \\
\hline FL & USGS-FLWSC & P-0736 MIDDLE RD UFA & 292124081345202 & - & Putnam & 292125 & 813451 & $5 / 25 / 2010$ & 8 & \\
\hline FL & USGS-FLWSC & $\begin{array}{l}\text { P-0817 UFA NR LK BRO- } \\
\text { WARD }\end{array}$ & 293206081351701 & - & Putnam & 293207 & 813516 & $5 / 25 / 2010$ & 25 & \\
\hline FL & USGS-FLWSC & $\begin{array}{l}\text { P-0822 FLORIDA ROCK } \\
\text { GRANDIN UFA }\end{array}$ & 294243081555901 & - & Putnam & 294244 & 815558 & $5 / 25 / 2010$ & 81 & \\
\hline FL & USGS-FLWSC & $\begin{array}{l}\text { P-0891 E H MILLER } \\
\text { SCHOOL UFA }\end{array}$ & 293755081412903 & - & Putnam & 293756 & 814128 & $5 / 25 / 2010$ & 28 & \\
\hline FL & USGS-FLWSC & $\begin{array}{l}\text { P-2037 LAKE STELLA AT } \\
\text { CRESCENT CITY }\end{array}$ & 292555081305003 & - & Putnam & 292608 & 813050 & $5 / 25 / 2010$ & 23 & \\
\hline FL & USGS-FLWSC & $\begin{array}{l}\text { P-4083 ETONIA MANNING } \\
\text { TRACT }\end{array}$ & 294816081482201 & - & Putnam & 294817 & 814821 & $5 / 25 / 2010$ & 72 & \\
\hline FL & USGS-FLWSC & $\begin{array}{l}\text { P-4086 ETONIA EAST V } \\
\text { ROAD }\end{array}$ & 294321081492103 & - & Putnam & 294322 & 814920 & $5 / 25 / 2010$ & 73 & \\
\hline FL & USGS-FLWSC & $\begin{array}{l}\text { Pasco Co. 24" UFA \#42 near } \\
\text { Land 0' Lakes }\end{array}$ & 281035082305701 & - & Pasco & 281036 & 823056 & $5 / 18 / 2010$ & 48 & \\
\hline FL & USGS-FLWSC & $\begin{array}{l}\text { Pasco Co. 6" UFA \#13 near } \\
\text { Drexel }\end{array}$ & 281558082264601 & - & Pasco & 281559 & 822645 & $5 / 18 / 2010$ & 73 & \\
\hline FL & USGS-FLWSC & $\begin{array}{l}\text { PBF-1 BROADVIEW CON- } \\
\text { DOS }\end{array}$ & 265811080051601 & - & Palm Beach & 265812 & 800515 & $5 / 18 / 2010$ & 47 & $\begin{array}{l}\text { Uncorrected hydrau- } \\
\text { lic head reported; } \\
\text { brackish to saline } \\
\text { water; not con- } \\
\text { toured on Potentio- } \\
\text { metric surface map }\end{array}$ \\
\hline FL & USGS-FLWSC & $\begin{array}{l}\text { Peace River Ranch 10" UFA } \\
\quad \# 231 \text { near Zolfo Springs }\end{array}$ & 272855081400701 & - & Hardee & 272855 & 814007 & $5 / 18 / 2010$ & 59 & \\
\hline FL & USGS-FLWSC & Pin Vtech TR13-2A 6" UFA & 275430082431402 & - & Pinellas & 275431 & 824313 & $5 / 17 / 2010$ & 5 & \\
\hline
\end{tabular}


Table 1. Water-level measurements in Florida and parts of Georgia, South Carolina, and Alabama, May-June 2010.—Continued

[NGVD 29, National Geodetic Vertical Datum of 1929. Abbreviations for agency database: ACEPD, Alachua County Environmental Protection Department; FGS, Florida Geological Survey; GSA, Geological Survey of Alabama; NWFWMD, Northwest Florida Water Management District; SFWMD, South Florida Water Management District; SRWMD, Suwannee River Water Management District; USGSALWSC, U.S. Geological Survey Alabama Water Science Center; USGS-FLWSC, U.S. Geological Survey Florida Water Science Center; GAWSC, U.S. Geological Survey Georgia Water Science Center; SCWSC, U.S. Geological Survey South Carolina Water Science Center. Abbreviations for Station names: BLVD., boulevard; CO, county; CR, county road; E, east; HS, high school; IFAS, Institute of Food and Agricultural Services; LK, lake; MW, monitoring well; N, north; NE, northeast; NE, northeast; NF, national forest; NW, northwest; PK, park; R., river; RD., road; SE, southeast; SE, southeast; SR, state road; ST., street; TWR, tower; UFA, Upper Floridan aquifer; —, not available; \#, number; “, inches]

\begin{tabular}{|c|c|c|c|c|c|c|c|c|c|c|}
\hline State & $\begin{array}{c}\text { Agency } \\
\text { database }\end{array}$ & Station name & $\begin{array}{c}\text { USGS site } \\
\text { identification } \\
\text { number }\end{array}$ & $\begin{array}{l}\text { Local agency } \\
\text { unique site } \\
\text { identifier }\end{array}$ & County & $\begin{array}{l}\text { Latitude } \\
\text { (degree } \\
\text { minutes } \\
\text { and } \\
\text { seconds) } \\
\text { [DDMMSS] }\end{array}$ & $\begin{array}{l}\text { Longitude } \\
\text { (degree } \\
\text { minutes } \\
\text { and } \\
\text { seconds) } \\
\text { [DDMMSS] }\end{array}$ & $\begin{array}{l}\text { Date of } \\
\text { measure- } \\
\text { ment }\end{array}$ & $\begin{array}{c}\text { Water } \\
\text { level } \\
\text { altitude } \\
\text { (feet } \\
\text { above } \\
\text { NGVD 29) }\end{array}$ & Remarks \\
\hline FL & USGS-FLWSC & $\begin{array}{l}\text { PINE CASTLE PO AT OR- } \\
\text { ANGE AVE }\end{array}$ & 282718081215101 & - & Orange & 282719 & 812150 & $5 / 17 / 2010$ & 47 & \\
\hline FL & USGS-FLWSC & $\begin{array}{l}\text { Pinellas 10" UFA \#665 near } \\
\text { Clearwater }\end{array}$ & 275815082440401 & - & Pinellas & 275816 & 824403 & $5 / 18 / 2010$ & 9 & \\
\hline FL & USGS-FLWSC & $\begin{array}{l}\text { PITMAN WORKCENTER } \\
\text { UFA-USFS }\end{array}$ & 290000081380001 & - & Lake & 290045 & 813827 & $5 / 26 / 2010$ & 47 & \\
\hline FL & USGS-FLWSC & $\begin{array}{l}\text { Plant HS Stadium 6" UFA, } \\
\text { Tampa }\end{array}$ & 275526082301301 & - & Hillsborough & 275527 & 823012 & $5 / 20 / 2010$ & 10 & \\
\hline FL & USGS-FLWSC & $\begin{array}{l}\text { PO0001 THORNHILL 4" UFA } \\
\text { NR DAVENPORT }\end{array}$ & 281202081391701 & - & Polk & 281203 & 813846 & $5 / 17 / 2010$ & 126 & \\
\hline FL & USGS-FLWSC & $\begin{array}{l}\text { PO0006 LK ALFRED 6" UFA } \\
\text { NR LK ALFRED }\end{array}$ & 281008081441801 & - & Polk & 281009 & 814417 & $5 / 17 / 2010$ & 127 & \\
\hline FL & USGS-FLWSC & $\begin{array}{l}\text { Ponderosa Club CE30A 2" UFA } \\
\text { Artesian (M-0316) near Lisk } \\
\text { Point }\end{array}$ & 291728081390501 & - & Marion & 291729 & 813904 & $5 / 25 / 2010$ & 16 & \\
\hline FL & USGS-FLWSC & $\begin{array}{l}\text { Ponderosa Dev 2" UFA, Bayo- } \\
\text { net Point }\end{array}$ & 281954082413401 & - & Pasco & 281955 & 824133 & $5 / 19 / 2010$ & 2 & \\
\hline FL & USGS-FLWSC & $\begin{array}{l}\text { Progress Energy MZ-3 Deep } \\
\text { Well near Crystal River, FL }\end{array}$ & 285738082400601 & - & Citrus & 285738 & 824006 & $5 / 18 / 2010$ & 5 & \\
\hline FL & USGS-FLWSC & $\begin{array}{l}\text { Pulis Tamp 4" UFA near Citrus } \\
\text { Park }\end{array}$ & 280354082335501 & - & Hillsborough & 280355 & 823354 & $5 / 19 / 2010$ & 22 & \\
\hline FL & USGS-FLWSC & Pz-1 6" UFA near Elfers & 281420082344701 & - & Pasco & 281421 & 823446 & $5 / 20 / 2010$ & 39 & \\
\hline FL & USGS-FLWSC & Pz-3 6" UFA near Elfers & 281457082334301 & - & Pasco & 281458 & 823342 & $5 / 20 / 2010$ & 44 & \\
\hline FL & USGS-FLWSC & Pz-4 6" UFA near Elfers & 281531082352901 & - & Pasco & 281532 & 823528 & $5 / 20 / 2010$ & 37 & \\
\hline FL & USGS-FLWSC & Pz-5 6" UFA near Elfers & 281458082330701 & - & Pasco & 281459 & 823306 & $5 / 20 / 2010$ & 44 & \\
\hline FL & USGS-FLWSC & $\begin{array}{l}\text { QW OBS WELL CE13 NR } \\
\text { RAINBOW SPGS }\end{array}$ & 290447082250901 & - & Marion & 290448 & 822508 & $5 / 24 / 2010$ & 36 & \\
\hline
\end{tabular}


Table 1. Water-level measurements in Florida and parts of Georgia, South Carolina, and Alabama, May-June 2010.-Continued

[NGVD 29, National Geodetic Vertical Datum of 1929. Abbreviations for agency database: ACEPD, Alachua County Environmental Protection Department; FGS, Florida Geological Survey; GSA, Geological Survey of Alabama; NWFWMD, Northwest Florida Water Management District; SFWMD, South Florida Water Management District; SRWMD, Suwannee River Water Management District; USGSALWSC, U.S. Geological Survey Alabama Water Science Center; USGS-FLWSC, U.S. Geological Survey Florida Water Science Center; GAWSC, U.S. Geological Survey Georgia Water Science Center; SCWSC, U.S. Geological Survey South Carolina Water Science Center. Abbreviations for Station names: BLVD., boulevard; CO, county; CR, county road; E, east; HS, high school; IFAS, Institute of Food and Agricultural Services; LK, lake; MW, monitoring well; N, north; NE, northeast; NE, northeast; NF, national forest; NW, northwest; PK, park; R., river; RD., road; SE, southeast; SE, southeast; SR, state road; ST., street; TWR, tower; UFA, Upper Floridan aquifer; - - not available; \#, number; “, inches]

\begin{tabular}{|c|c|c|c|c|c|c|c|c|c|c|}
\hline State & $\begin{array}{l}\text { Agency } \\
\text { database }\end{array}$ & Station name & $\begin{array}{c}\text { USGS site } \\
\text { identification } \\
\text { number }\end{array}$ & $\begin{array}{c}\text { Local agency } \\
\text { unique site } \\
\text { identifier }\end{array}$ & County & $\begin{array}{l}\text { Latitude } \\
\text { (degree } \\
\text { minutes } \\
\text { and } \\
\text { seconds) } \\
\text { [DDMMSS] }\end{array}$ & $\begin{array}{l}\text { Longitude } \\
\text { (degree } \\
\text { minutes } \\
\text { and } \\
\text { seconds) } \\
\text { [DDMMSS] }\end{array}$ & $\begin{array}{c}\text { Date of } \\
\text { measure- } \\
\text { ment }\end{array}$ & $\begin{array}{c}\text { Water } \\
\text { level } \\
\text { altitude } \\
\text { (feet } \\
\text { above } \\
\text { NGVD 29) }\end{array}$ & Remarks \\
\hline FL & USGS-FLWSC & $\begin{array}{l}\text { QW OBS WELL CE77 NR } \\
\text { DUNNELLON }\end{array}$ & 290216082292001 & - & Citrus & 290217 & 822919 & $5 / 20 / 2010$ & 13 & \\
\hline FL & USGS-FLWSC & $\begin{array}{l}\text { QW UFA CE22 NR DUNNEL- } \\
\text { LON }\end{array}$ & 290312082190601 & - & Marion & 290313 & 821905 & $5 / 24 / 2010$ & 49 & \\
\hline FL & USGS-FLWSC & $\begin{array}{l}\text { RAINBOW SPG WELL NR } \\
\text { DUNNELLON }\end{array}$ & 290514082270701 & - & Marion & 290508 & 822707 & $5 / 24 / 2010$ & 31 & \\
\hline FL & USGS-FLWSC & $\begin{array}{l}\text { REEDY CR OVERLOOK 6" } \\
\text { UFA NR SOUTH }\end{array}$ & 280905081270101 & - & Osceola & 280906 & 812700 & $5 / 17 / 2010$ & 60 & \\
\hline FL & USGS-FLWSC & $\begin{array}{l}\text { Regency Oaks Well Nr Safety } \\
\text { Harbor }\end{array}$ & 275949082442401 & - & Pinellas & 275950 & 824324 & $5 / 19 / 2010$ & 8 & \\
\hline FL & USGS-FLWSC & $\begin{array}{l}\text { RICHLOAM FIRE TOWER } \\
\text { WELL }\end{array}$ & 283001082064702 & - & Hernando & 283002 & 820646 & $5 / 18 / 2010$ & 74 & \\
\hline FL & USGS-FLWSC & RIDGE MANOR ROMP 99 & 283036082105501 & - & Hernando & 283037 & 821054 & $5 / 18 / 2010$ & 52 & \\
\hline FL & USGS-FLWSC & $\begin{array}{l}\text { RIVER RANCH 4" UFA NR } \\
\text { INDIAN LAKES ESTATES }\end{array}$ & 274815081130301 & - & Polk & 274816 & 811302 & $5 / 18 / 2010$ & 46 & \\
\hline FL & USGS-FLWSC & $\begin{array}{l}\text { RIVER RANCH REPLACE- } \\
\text { MENT }\end{array}$ & 274552081115201 & - & Polk & 274553 & 811151 & $5 / 18 / 2010$ & 43 & \\
\hline FL & USGS-FLWSC & Rivercrest 8" UFA, Riverview & 275130082194501 & - & Hillsborough & 275131 & 821944 & $5 / 19 / 2010$ & 12 & \\
\hline FL & USGS-FLWSC & $\begin{array}{l}\text { ROBINSON 4" UFA, SR } 337 \\
\text { AND LCR } 158\end{array}$ & 291712082351801 & - & Levy & 291706 & 823518 & $5 / 26 / 2010$ & 46 & \\
\hline FL & USGS-FLWSC & $\begin{array}{l}\text { Robinson HS Stadium 4" UFA } \\
\text { near Tampa }\end{array}$ & 275227082310101 & - & Hillsborough & 275228 & 823100 & $5 / 20 / 2010$ & 1 & \\
\hline FL & USGS-FLWSC & $\begin{array}{l}\text { RODEO FIELD DEER PARK } \\
\text { NW }\end{array}$ & 281105080541401 & - & Osceola & 281106 & 805413 & $5 / 18 / 2010$ & 39 & \\
\hline FL & USGS-FLWSC & $\begin{array}{l}\text { ROMP } 18 \text { " UFA near Citrus } \\
\text { Park }\end{array}$ & 280901082310401 & - & Hillsborough & 280902 & 823103 & $5 / 19 / 2010$ & 47 & \\
\hline FL & USGS-FLWSC & ROMP 101 UFA NR BAY LK & 282717081553101 & - & Lake & 282718 & 815530 & $5 / 20 / 2010$ & 98 & \\
\hline
\end{tabular}


Table 1. Water-level measurements in Florida and parts of Georgia, South Carolina, and Alabama, May-June 2010.—Continued

[NGVD 29, National Geodetic Vertical Datum of 1929. Abbreviations for agency database: ACEPD, Alachua County Environmental Protection Department; FGS, Florida Geological Survey; GSA, Geological Survey of Alabama; NWFWMD, Northwest Florida Water Management District; SFWMD, South Florida Water Management District; SRWMD, Suwannee River Water Management District; USGSALWSC, U.S. Geological Survey Alabama Water Science Center; USGS-FLWSC, U.S. Geological Survey Florida Water Science Center; GAWSC, U.S. Geological Survey Georgia Water Science Center; SCWSC, U.S. Geological Survey South Carolina Water Science Center. Abbreviations for Station names: BLVD., boulevard; CO, county; CR, county road; E, east; HS, high school; IFAS, Institute of Food and Agricultural Services; LK, lake; MW, monitoring well; N, north; NE, northeast; NE, northeast; NF, national forest; NW, northwest; PK, park; R., river; RD., road; SE, southeast; SE, southeast; SR, state road; ST., street; TWR, tower; UFA, Upper Floridan aquifer; —, not available; \#, number; “, inches]

\begin{tabular}{|c|c|c|c|c|c|c|c|c|c|c|}
\hline State & $\begin{array}{c}\text { Agency } \\
\text { database }\end{array}$ & Station name & $\begin{array}{c}\text { USGS site } \\
\text { identification } \\
\text { number }\end{array}$ & $\begin{array}{l}\text { Local agency } \\
\text { unique site } \\
\text { identifier }\end{array}$ & County & $\begin{array}{l}\text { Latitude } \\
\text { (degree } \\
\text { minutes } \\
\text { and } \\
\text { seconds) } \\
\text { [DDMMSS] }\end{array}$ & $\begin{array}{l}\text { Longitude } \\
\text { (degree } \\
\text { minutes } \\
\text { and } \\
\text { seconds) } \\
\text { [DDMMSS] }\end{array}$ & $\begin{array}{c}\text { Date of } \\
\text { measure- } \\
\text { ment }\end{array}$ & $\begin{array}{c}\text { Water } \\
\text { level } \\
\text { altitude } \\
\text { (feet } \\
\text { above } \\
\text { NGVD 29) }\end{array}$ & Remarks \\
\hline FL & USGS-FLWSC & $\begin{array}{l}\text { ROMP } 103 \text { NR BROOKS- } \\
\text { VILLE }\end{array}$ & 283537082151501 & - & Hernando & 283538 & 821514 & $5 / 18 / 2010$ & 39 & \\
\hline FL & USGS-FLWSC & $\begin{array}{l}\text { ROMP } 105 \text { 6" UFA, Brooks- } \\
\text { ville }\end{array}$ & 283321082241601 & - & Hernando & 283322 & 822415 & $5 / 17 / 2010$ & 32 & \\
\hline FL & USGS-FLWSC & $\begin{array}{l}\text { ROMP } 107 \text { 6" UFA near } \\
\text { Brooksville }\end{array}$ & 283924082272301 & - & Hernando & 283925 & 822722 & $5 / 17 / 2010$ & 11 & \\
\hline FL & USGS-FLWSC & ROMP 109 N STOREY MINE & 284330082215401 & - & Citrus & 284331 & 822153 & $5 / 21 / 2010$ & 16 & \\
\hline FL & USGS-FLWSC & $\begin{array}{l}\text { ROMP } 113 \text { REPLACEMENT } \\
\text { NR INVERNESS }\end{array}$ & 285121082245401 & - & Citrus & 285122 & 822453 & $5 / 19 / 2010$ & 7 & \\
\hline FL & USGS-FLWSC & $\begin{array}{l}\text { ROMP } 12 \text { Nocatee } 8 " \text { UFA \#5 } \\
\text { near Arcadia }\end{array}$ & 270225081443303 & - & De Soto & 270226 & 814432 & $5 / 18 / 2010$ & 46 & \\
\hline FL & USGS-FLWSC & $\begin{array}{l}\text { ROMP } 1238 \text { " UFA near } \\
\text { Wimauma }\end{array}$ & 274031082150401 & - & Hillsborough & 274032 & 821503 & $5 / 18 / 2010$ & -1 & \\
\hline FL & USGS-FLWSC & $\begin{array}{l}\text { ROMP } 124 \text { D 8" UFA NR } \\
\text { YANKEETOWN }\end{array}$ & 290200082432301 & - & Levy & 290201 & 824322 & $5 / 26 / 2010$ & 3 & \\
\hline FL & USGS-FLWSC & $\begin{array}{l}\text { ROMP } 125 \text { D 6" UFA, } \\
\text { CRACKERTOWN }\end{array}$ & 290230082412501 & - & Levy & 290231 & 824124 & $5 / 26 / 2010$ & 4 & \\
\hline FL & USGS-FLWSC & $\begin{array}{l}\text { ROMP } 13 \text { (MW-4) UFA near } \\
\quad \text { Arcadia }\end{array}$ & 270418081365802 & - & De Soto & 270419 & 813657 & $5 / 18 / 2010$ & 48 & \\
\hline FL & USGS-FLWSC & $\begin{array}{l}\text { ROMP } 13 \text { 6" UFA near Citrus } \\
\text { Park }\end{array}$ & 280155082340001 & - & Hillsborough & 280156 & 823359 & $5 / 19 / 2010$ & 17 & \\
\hline FL & USGS-FLWSC & $\begin{array}{l}\text { ROMP } 134 \text { 6" UFA NR WIL- } \\
\text { LISTON }\end{array}$ & 292615082272601 & - & Levy & 292616 & 822725 & $5 / 26 / 2010$ & 45 & \\
\hline FL & USGS-FLWSC & $\begin{array}{l}\text { ROMP } 14 \text { Suwanee Well near } \\
\text { Lake Placid }\end{array}$ & 270858081211104 & - & Highlands & 270859 & 812110 & $5 / 19 / 2010$ & 48 & \\
\hline FL & USGS-FLWSC & $\begin{array}{l}\text { ROMP } 15 \text { 10" UFA near } \\
\text { Arcadia }\end{array}$ & 271232081392201 & - & De Soto & 271233 & 813921 & $5 / 20 / 2010$ & 47 & \\
\hline
\end{tabular}


Table 1. Water-level measurements in Florida and parts of Georgia, South Carolina, and Alabama, May-June 2010.-Continued

[NGVD 29, National Geodetic Vertical Datum of 1929. Abbreviations for agency database: ACEPD, Alachua County Environmental Protection Department; FGS, Florida Geological Survey; GSA, Geological Survey of Alabama; NWFWMD, Northwest Florida Water Management District; SFWMD, South Florida Water Management District; SRWMD, Suwannee River Water Management District; USGSALWSC, U.S. Geological Survey Alabama Water Science Center; USGS-FLWSC, U.S. Geological Survey Florida Water Science Center; GAWSC, U.S. Geological Survey Georgia Water Science Center; SCWSC, U.S. Geological Survey South Carolina Water Science Center. Abbreviations for Station names: BLVD., boulevard; CO, county; CR, county road; E, east; HS, high school; IFAS, Institute of Food and Agricultural Services; LK, lake; MW, monitoring well; N, north; NE, northeast; NE, northeast; NF, national forest; NW, northwest; PK, park; R., river; RD., road; SE, southeast; SE, southeast; SR, state road; ST., street; TWR, tower; UFA, Upper Floridan aquifer; - - not available; \#, number; “, inches]

\begin{tabular}{|c|c|c|c|c|c|c|c|c|c|c|}
\hline State & $\begin{array}{c}\text { Agency } \\
\text { database }\end{array}$ & Station name & $\begin{array}{c}\text { USGS site } \\
\text { identification } \\
\text { number }\end{array}$ & $\begin{array}{l}\text { Local agency } \\
\text { unique site } \\
\text { identifier }\end{array}$ & County & $\begin{array}{l}\text { Latitude } \\
\text { (degree } \\
\text { minutes } \\
\text { and } \\
\text { seconds) } \\
\text { [DDMMSS] }\end{array}$ & $\begin{array}{l}\text { Longitude } \\
\text { (degree } \\
\text { minutes } \\
\text { and } \\
\text { seconds) } \\
\text { [DDMMSS] }\end{array}$ & $\begin{array}{c}\text { Date of } \\
\text { measure- } \\
\text { ment }\end{array}$ & $\begin{array}{l}\text { Water } \\
\text { level } \\
\text { altitude } \\
\text { (feet } \\
\text { above } \\
\text { NGVD 29) }\end{array}$ & Remarks \\
\hline FL & USGS-FLWSC & $\begin{array}{l}\text { ROMP } 15 \text { 6" UFA near Citrus } \\
\text { Park }\end{array}$ & 275955082340001 & - & Hillsborough & 275956 & 823359 & $5 / 19 / 2010$ & 8 & \\
\hline FL & USGS-FLWSC & ROMP 16 6" UFA near Arcadia & 271115081462701 & - & De Soto & 271116 & 814626 & $5 / 20 / 2010$ & 46 & \\
\hline FL & USGS-FLWSC & $\begin{array}{l}\text { Romp 16.5 Fort Ogden Avon } \\
\text { Park Well near Arcadia, FL }\end{array}$ & 270340081530201 & - & De Soto & 270340 & 815302 & $5 / 19 / 2010$ & 47 & \\
\hline FL & USGS-FLWSC & ROMP 17 6" UFA near Arcadia & 271026081583601 & - & De Soto & 271027 & 815835 & $5 / 20 / 2010$ & 35 & \\
\hline FL & USGS-FLWSC & $\begin{array}{l}\text { ROMP 18-1 6" UFA near } \\
\text { Arcadia }\end{array}$ & 271137082074801 & - & Sarasota & 271138 & 820747 & $5 / 18 / 2010$ & 36 & \\
\hline FL & USGS-FLWSC & $\begin{array}{c}\text { ROMP } 19 \text { (City Of St. Pete \#E- } \\
\text { 103) 6" UFA near Oldsmar }\end{array}$ & 280603082385401 & - & Hillsborough & 280604 & 823853 & $5 / 19 / 2010$ & 20 & \\
\hline FL & USGS-FLWSC & $\begin{array}{l}\text { ROMP } 19 \text { Wlam UFA near } \\
\text { Sarasota }\end{array}$ & 270959082203001 & - & Sarasota & 271000 & 822029 & $5 / 18 / 2010$ & 24 & \\
\hline FL & USGS-FLWSC & $\begin{array}{l}\text { ROMP } 20 \text { OB-3 6" UFA, } \\
\text { Osprey }\end{array}$ & 271137082284501 & - & Sarasota & 271138 & 822844 & $5 / 19 / 2010$ & 20 & $\begin{array}{l}\text { Uncorrected hydrau- } \\
\text { lic head reported; } \\
\text { brackish to saline } \\
\text { water; not con- } \\
\text { toured on Potentio- } \\
\text { metric surface map }\end{array}$ \\
\hline FL & USGS-FLWSC & $\begin{array}{l}\text { ROMP } 22 \text { 6" UFA near Fruit- } \\
\text { ville }\end{array}$ & 271813082201301 & - & Sarasota & 271814 & 822012 & $5 / 19 / 2010$ & 9 & \\
\hline FL & USGS-FLWSC & $\begin{array}{l}\text { ROMP } 23 \text { 6" UFA near Myakka } \\
\text { City }\end{array}$ & 271906082112401 & - & Manatee & 271907 & 821123 & $5 / 18 / 2010$ & 16 & \\
\hline FL & USGS-FLWSC & $\begin{array}{l}\text { Romp } 25 \text { Lily Avon Park Well } \\
\text { near Zolfo Springs, FL }\end{array}$ & 272159082002502 & - & Hardee & 272159 & 820025 & $5 / 17 / 2010$ & 19 & \\
\hline FL & USGS-FLWSC & ROMP 26 UFA near Gardner & 271757081493002 & - & De Soto & 271758 & 814929 & $5 / 21 / 2010$ & 43 & \\
\hline FL & USGS-FLWSC & $\begin{array}{l}\text { ROMP } 28 \text { (Kuhlman) (12101 } \\
\text { and 1042) UFA }\end{array}$ & 272207081260401 & - & Highlands & 272208 & 812603 & $5 / 19 / 2010$ & 59 & \\
\hline
\end{tabular}


Table 1. Water-level measurements in Florida and parts of Georgia, South Carolina, and Alabama, May-June 2010.—Continued

[NGVD 29, National Geodetic Vertical Datum of 1929. Abbreviations for agency database: ACEPD, Alachua County Environmental Protection Department; FGS, Florida Geological Survey; GSA, Geological Survey of Alabama; NWFWMD, Northwest Florida Water Management District; SFWMD, South Florida Water Management District; SRWMD, Suwannee River Water Management District; USGSALWSC, U.S. Geological Survey Alabama Water Science Center; USGS-FLWSC, U.S. Geological Survey Florida Water Science Center; GAWSC, U.S. Geological Survey Georgia Water Science Center; SCWSC, U.S. Geological Survey South Carolina Water Science Center. Abbreviations for Station names: BLVD., boulevard; CO, county; CR, county road; E, east; HS, high school; IFAS, Institute of Food and Agricultural Services; LK, lake; MW, monitoring well; N, north; NE, northeast; NE, northeast; NF, national forest; NW, northwest; PK, park; R., river; RD., road; SE, southeast; SE, southeast; SR, state road; ST., street; TWR, tower; UFA, Upper Floridan aquifer; —, not available; \#, number; “, inches]

\begin{tabular}{|c|c|c|c|c|c|c|c|c|c|c|}
\hline State & $\begin{array}{l}\text { Agency } \\
\text { database }\end{array}$ & Station name & $\begin{array}{c}\text { USGS site } \\
\text { identification } \\
\text { number }\end{array}$ & $\begin{array}{l}\text { Local agency } \\
\text { unique site } \\
\text { identifier }\end{array}$ & County & $\begin{array}{l}\text { Latitude } \\
\text { (degree } \\
\text { minutes } \\
\text { and } \\
\text { seconds) } \\
\text { [DDMMSS] }\end{array}$ & $\begin{array}{l}\text { Longitude } \\
\text { (degree } \\
\text { minutes } \\
\text { and } \\
\text { seconds) } \\
\text { [DDMMSS] }\end{array}$ & $\begin{array}{l}\text { Date of } \\
\text { measure- } \\
\text { ment }\end{array}$ & $\begin{array}{c}\text { Water } \\
\text { level } \\
\text { altitude } \\
\text { (feet } \\
\text { above } \\
\text { NGVD 29) }\end{array}$ & Remarks \\
\hline FL & USGS-FLWSC & $\begin{array}{l}\text { ROMP 28X 8" UFA near Lake } \\
\text { Placid }\end{array}$ & 271559081202301 & - & Highlands & 271600 & 812022 & $5 / 19 / 2010$ & 67 & \\
\hline FL & USGS-FLWSC & $\begin{array}{l}\text { ROMP } 3 \text { 6" UFA near Citrus } \\
\text { Park }\end{array}$ & 280819082374301 & - & Hillsborough & 280820 & 823742 & $5 / 19 / 2010$ & 27 & \\
\hline FL & USGS-FLWSC & $\begin{array}{l}\text { ROMP } 30 \text { (30-1) 8" UFA near } \\
\text { Zolfo Springs }\end{array}$ & 272728081474701 & - & Hardee & 272729 & 814746 & $5 / 20 / 2010$ & 42 & \\
\hline FL & USGS-FLWSC & ROMP 31 8" UFA near Ona & 272714081545901 & - & Hardee & 272715 & 815458 & $5 / 21 / 2010$ & 36 & \\
\hline FL & USGS-FLWSC & $\begin{array}{l}\text { ROMP } 32 \text { 8" UFA near Myakka } \\
\text { Head }\end{array}$ & 272814082034802 & - & Manatee & 272815 & 820347 & $5 / 17 / 2010$ & 14 & \\
\hline FL & USGS-FLWSC & $\begin{array}{l}\text { ROMP } 33 \text { 12" UFA near } \\
\text { Bethany }\end{array}$ & 272728082153002 & - & Manatee & 272729 & 821529 & $5 / 17 / 2010$ & -5 & \\
\hline FL & USGS-FLWSC & $\begin{array}{l}\text { Romp } 35 \text { Suwannee Well near } \\
\text { Arcadia, FL }\end{array}$ & 271705082022101 & - & De Soto & 271707 & 820227 & $5 / 20 / 2010$ & 30 & \\
\hline FL & USGS-FLWSC & ROMP 39 6" UFA near Parrish & 273521082150501 & - & Manatee & 273522 & 821504 & $5 / 18 / 2010$ & -3 & \\
\hline FL & USGS-FLWSC & $\begin{array}{l}\text { ROMP 40-1 (GD-11) 10" UFA } \\
\text { near Duette }\end{array}$ & 273851082031501 & - & Polk & 273852 & 820314 & $5 / 19 / 2010$ & 33 & \\
\hline FL & USGS-FLWSC & $\begin{array}{l}\text { ROMP } 43 \text { 8" UFA near Avon } \\
\quad \text { Park }\end{array}$ & 273615081284901 & - & Highlands & 273616 & 812848 & $5 / 19 / 2010$ & 82 & \\
\hline FL & USGS-FLWSC & $\begin{array}{l}\text { Romp } 43 \text { MW-4 Suwannee } \\
\text { Well Nr Avon Park, FL }\end{array}$ & 273501081351902 & - & Hardee & 273500 & 813519 & $5 / 18 / 2010$ & 72 & \\
\hline FL & USGS-FLWSC & $\begin{array}{l}\text { ROMP } 44 \text { 10" UFA near Lake } \\
\text { Wales }\end{array}$ & 274926081355301 & - & Polk & 274927 & 813552 & $5 / 18 / 2010$ & 86 & \\
\hline FL & USGS-FLWSC & ROMP 45 8" UFA, Ft. Meade & 274547081470903 & - & Polk & 274548 & 814708 & $5 / 18 / 2010$ & 67 & \\
\hline FL & USGS-FLWSC & $\begin{array}{l}\text { ROMP } 48 \text { 6" UFA near Ft. } \\
\text { Lonesome }\end{array}$ & 274427082083701 & - & Hillsborough & 274428 & 820836 & $5 / 18 / 2010$ & 27 & \\
\hline FL & USGS-FLWSC & ROMP 49 6" UFA near Balm & 274546082151403 & - & Hillsborough & 274547 & 821513 & $5 / 18 / 2010$ & 11 & \\
\hline
\end{tabular}


Table 1. Water-level measurements in Florida and parts of Georgia, South Carolina, and Alabama, May-June 2010.—Continued

[NGVD 29, National Geodetic Vertical Datum of 1929. Abbreviations for agency database: ACEPD, Alachua County Environmental Protection Department; FGS, Florida Geological Survey; GSA, Geological Survey of Alabama; NWFWMD, Northwest Florida Water Management District; SFWMD, South Florida Water Management District; SRWMD, Suwannee River Water Management District; USGSALWSC, U.S. Geological Survey Alabama Water Science Center; USGS-FLWSC, U.S. Geological Survey Florida Water Science Center; GAWSC, U.S. Geological Survey Georgia Water Science Center; SCWSC, U.S. Geological Survey South Carolina Water Science Center. Abbreviations for Station names: BLVD., boulevard; CO, county; CR, county road; E, east; HS, high school; IFAS, Institute of Food and Agricultural Services; LK, lake; MW, monitoring well; N, north; NE, northeast; NE, northeast; NF, national forest; NW, northwest; PK, park; R., river; RD., road; SE, southeast; SE, southeast; SR, state road; ST., street; TWR, tower; UFA, Upper Floridan aquifer; - - not available; \#, number; “, inches]

\begin{tabular}{|c|c|c|c|c|c|c|c|c|c|c|}
\hline State & $\begin{array}{c}\text { Agency } \\
\text { database }\end{array}$ & Station name & $\begin{array}{c}\text { USGS site } \\
\text { identification } \\
\text { number }\end{array}$ & $\begin{array}{c}\text { Local agency } \\
\text { unique site } \\
\text { identifier }\end{array}$ & County & $\begin{array}{l}\text { Latitude } \\
\text { (degree } \\
\text { minutes } \\
\text { and } \\
\text { seconds) } \\
\text { [DDMMSS] }\end{array}$ & $\begin{array}{l}\text { Longitude } \\
\text { (degree } \\
\text { minutes } \\
\text { and } \\
\text { seconds) } \\
\text { ] [DDMMSS] }\end{array}$ & $\begin{array}{c}\text { Date of } \\
\text { measure- } \\
\text { ment }\end{array}$ & $\begin{array}{l}\text { Water } \\
\text { level } \\
\text { altitude } \\
\text { (feet } \\
\text { above } \\
\text { NGVD 29) }\end{array}$ & Remarks \\
\hline FL & USGS-FLWSC & ROMP 5 (MW-4) UFA & 265644081483301 & - & Charlotte & 265644 & 814832 & $5 / 18 / 2010$ & 48 & $\begin{array}{l}\text { Uncorrected hydrau- } \\
\text { lic head reported; } \\
\text { brackish to saline } \\
\text { water; not con- } \\
\text { toured on Potentio- } \\
\text { metric surface map }\end{array}$ \\
\hline FL & USGS-FLWSC & $\begin{array}{l}\text { ROMP } 5 \text { 6" UFA near Citrus } \\
\text { Park }\end{array}$ & 280602082333001 & - & Hillsborough & 280603 & 823329 & $5 / 19 / 2010$ & 31 & \\
\hline FL & USGS-FLWSC & $\begin{array}{l}\text { ROMP } 50 \text { 6" UFA near Sun } \\
\text { City }\end{array}$ & 274240082212701 & - & Hillsborough & 274241 & 822126 & $5 / 19 / 2010$ & 3 & \\
\hline FL & USGS-FLWSC & $\begin{array}{l}\text { ROMP } 55 \text { 10" UFA near Bab- } \\
\text { son Park }\end{array}$ & 274730081333801 & - & Polk & 274731 & 813337 & $5 / 18 / 2010$ & 80 & \\
\hline FL & USGS-FLWSC & $\begin{array}{l}\text { ROMP } 57 \text { 6" UFA near Lake } \\
\text { Wales }\end{array}$ & 275411081372001 & - & Polk & 275412 & 813719 & $5 / 17 / 2010$ & 102 & \\
\hline FL & USGS-FLWSC & $\begin{array}{l}\text { ROMP 57A 6" UFA, Lake } \\
\text { Wales }\end{array}$ & 275348081335701 & - & Polk & 275349 & 813356 & $5 / 17 / 2010$ & 98 & \\
\hline FL & USGS-FLWSC & $\begin{array}{l}\text { ROMP } 58 \text { 10" UFA, Lake } \\
\text { Wales }\end{array}$ & 275507081353701 & - & Polk & 275508 & 813536 & $5 / 17 / 2010$ & 96 & \\
\hline FL & USGS-FLWSC & ROMP 59 12" UFA, Bartow & 275314081514201 & - & Polk & 275315 & 815141 & $6 / 1 / 2010$ & 68 & \\
\hline FL & USGS-FLWSC & ROMP 60 10" UFA, Mulberry & 275326081585801 & - & Polk & 275327 & 815857 & $5 / 19 / 2010$ & 67 & \\
\hline FL & USGS-FLWSC & $\begin{array}{l}\text { ROMP 60X 20" UFA near } \\
\text { Lakeland }\end{array}$ & 275728081570001 & - & Polk & 275729 & 815659 & $5 / 20 / 2010$ & 71 & \\
\hline FL & USGS-FLWSC & $\begin{array}{l}\text { ROMP } 61 \text { 12" UFA, Pleasant } \\
\text { Grove }\end{array}$ & 275429082093901 & - & Hillsborough & 275430 & 820938 & $5 / 17 / 2010$ & 37 & \\
\hline FL & USGS-FLWSC & $\begin{array}{l}\text { ROMP } 67 \text { Golden Gate Lime- } \\
\text { stone UFA }\end{array}$ & 280320082203801 & - & Hillsborough & 280321 & 822037 & $5 / 19 / 2010$ & 24 & \\
\hline FL & USGS-FLWSC & $\begin{array}{l}\text { ROMP 68-2 6" UFA near } \\
\text { Antioch }\end{array}$ & 280503082143702 & - & Hillsborough & 280504 & 821436 & $5 / 17 / 2010$ & 47 & \\
\hline
\end{tabular}


Table 1. Water-level measurements in Florida and parts of Georgia, South Carolina, and Alabama, May-June 2010.—Continued

[NGVD 29, National Geodetic Vertical Datum of 1929. Abbreviations for agency database: ACEPD, Alachua County Environmental Protection Department; FGS, Florida Geological Survey; GSA, Geological Survey of Alabama; NWFWMD, Northwest Florida Water Management District; SFWMD, South Florida Water Management District; SRWMD, Suwannee River Water Management District; USGSALWSC, U.S. Geological Survey Alabama Water Science Center; USGS-FLWSC, U.S. Geological Survey Florida Water Science Center; GAWSC, U.S. Geological Survey Georgia Water Science Center; SCWSC, U.S. Geological Survey South Carolina Water Science Center. Abbreviations for Station names: BLVD., boulevard; CO, county; CR, county road; E, east; HS, high school; IFAS, Institute of Food and Agricultural Services; LK, lake; MW, monitoring well; N, north; NE, northeast; NE, northeast; NF, national forest; NW, northwest; PK, park; R., river; RD., road; SE, southeast; SE, southeast; SR, state road; ST., street; TWR, tower; UFA, Upper Floridan aquifer; —, not available; \#, number; “, inches]

\begin{tabular}{|c|c|c|c|c|c|c|c|c|c|c|}
\hline State & $\begin{array}{l}\text { Agency } \\
\text { database }\end{array}$ & Station name & $\begin{array}{c}\text { USGS site } \\
\text { identification } \\
\text { number }\end{array}$ & $\begin{array}{l}\text { Local agency } \\
\text { unique site } \\
\text { identifier }\end{array}$ & County & $\begin{array}{l}\text { Latitude } \\
\text { (degree } \\
\text { minutes } \\
\text { and } \\
\text { seconds) } \\
\text { [DDMMSS] }\end{array}$ & $\begin{array}{l}\text { Longitude } \\
\text { (degree } \\
\text { minutes } \\
\text { and } \\
\text { seconds) } \\
\text { [DDMMSS] }\end{array}$ & $\begin{array}{l}\text { Date of } \\
\text { measure- } \\
\text { ment }\end{array}$ & $\begin{array}{c}\text { Water } \\
\text { level } \\
\text { altitude } \\
\text { (feet } \\
\text { above } \\
\text { NGVD 29) }\end{array}$ & Remarks \\
\hline FL & USGS-FLWSC & $\begin{array}{l}\text { ROMP } 7 \text { 6" UFA near Citrus } \\
\text { Park }\end{array}$ & 280449082303301 & - & Hillsborough & 280450 & 823032 & $5 / 19 / 2010$ & 38 & \\
\hline FL & USGS-FLWSC & $\begin{array}{l}\text { ROMP } 73 \text { 12" UFA, Winter- } \\
\text { haven }\end{array}$ & 280113081435301 & - & Polk & 280114 & 814352 & $5 / 20 / 2010$ & 114 & \\
\hline FL & USGS-FLWSC & $\begin{array}{l}\text { ROMP 76A 6" UFA, POLK } \\
\text { CITY }\end{array}$ & 281057081495002 & - & Polk & 281058 & 814949 & $5 / 19 / 2010$ & 128 & \\
\hline FL & USGS-FLWSC & $\begin{array}{l}\text { ROMP } 84 \text { FLRD Well near } \\
\text { Drexel, FL }\end{array}$ & 281435082260101 & - & Pasco & 281436 & 822600 & $5 / 17 / 2010$ & 64 & \\
\hline FL & USGS-FLWSC & $\begin{array}{l}\text { ROMP } 85 \text { 6" UFA near Zeph- } \\
\text { yrhills }\end{array}$ & 281424082192702 & - & Pasco & 281425 & 821926 & $5 / 17 / 2010$ & 78 & \\
\hline FL & USGS-FLWSC & $\begin{array}{l}\text { ROMP } 86 \text { 6" UFA near Zeph- } \\
\text { yrhills }\end{array}$ & 281504082104801 & - & Pasco & 281505 & 821047 & $5 / 17 / 2010$ & 65 & \\
\hline FL & USGS-FLWSC & ROMP 86A UFA & 281144082100402 & - & Pasco & 281145 & 821003 & $5 / 17 / 2010$ & 59 & \\
\hline FL & USGS-FLWSC & ROMP 87 NR LAKELAND & 281312082011601 & - & Polk & 281313 & 820115 & $5 / 17 / 2010$ & 103 & \\
\hline FL & USGS-FLWSC & ROMP 88 NR ROCKRIDGE & 281837081544101 & - & Polk & 281838 & 815440 & $5 / 17 / 2010$ & 104 & \\
\hline FL & USGS-FLWSC & $\begin{array}{l}\text { ROMP } 9 \text { (MW-6) 6" UFA } \\
\text { Monitor near NPort, Sarasota }\end{array}$ & 270432082085701 & - & Sarasota & 270433 & 820856 & $5 / 20 / 2010$ & 40 & \\
\hline FL & USGS-FLWSC & $\begin{array}{l}\text { ROMP 9.5 (MW-1) 22" UFA } \\
\text { near Ft. Ogden }\end{array}$ & 270737082025101 & - & De Soto & 270738 & 820250 & $5 / 19 / 2010$ & 42 & \\
\hline FL & USGS-FLWSC & $\begin{array}{l}\text { ROMP } 90 \text { AVON PARK WELL } \\
\text { NEAR DADE CITY FL }\end{array}$ & 282137082082302 & - & Pasco & 282138 & 820822 & $5 / 17 / 2010$ & 68 & \\
\hline FL & USGS-FLWSC & $\begin{array}{l}\text { ROMP } 93 \text { 6" UFA near Gowers } \\
\text { Corner }\end{array}$ & 281923082252201 & - & Pasco & 281924 & 822521 & $5 / 18 / 2010$ & 68 & \\
\hline FL & USGS-FLWSC & ROMP 97 6" UFA near Aripeka & 282605082345801 & - & Hernando & 282606 & 823457 & $5 / 17 / 2010$ & 16 & \\
\hline FL & USGS-FLWSC & ROMP BR-3 Lk Pasadena UFA & 281938082141501 & - & Pasco & 281939 & 821414 & $5 / 17 / 2010$ & 75 & \\
\hline FL & USGS-FLWSC & $\begin{array}{l}\text { ROMP Centralia 6" UFA near } \\
\text { Weeki Wachee }\end{array}$ & 283650082313301 & - & Hernando & 283651 & 823132 & $5 / 17 / 2010$ & 10 & \\
\hline
\end{tabular}


Table 1. Water-level measurements in Florida and parts of Georgia, South Carolina, and Alabama, May-June 2010.—Continued

[NGVD 29, National Geodetic Vertical Datum of 1929. Abbreviations for agency database: ACEPD, Alachua County Environmental Protection Department; FGS, Florida Geological Survey; GSA, Geological Survey of Alabama; NWFWMD, Northwest Florida Water Management District; SFWMD, South Florida Water Management District; SRWMD, Suwannee River Water Management District; USGSALWSC, U.S. Geological Survey Alabama Water Science Center; USGS-FLWSC, U.S. Geological Survey Florida Water Science Center; GAWSC, U.S. Geological Survey Georgia Water Science Center; SCWSC, U.S. Geological Survey South Carolina Water Science Center. Abbreviations for Station names: BLVD., boulevard; CO, county; CR, county road; E, east; HS, high school; IFAS, Institute of Food and Agricultural Services; LK, lake; MW, monitoring well; N, north; NE, northeast; NE, northeast; NF, national forest; NW, northwest; PK, park; R., river; RD., road; SE, southeast; SE, southeast; SR, state road; ST., street; TWR, tower; UFA, Upper Floridan aquifer; - - not available; \#, number; “, inches]

\begin{tabular}{|c|c|c|c|c|c|c|c|c|c|c|}
\hline State & $\begin{array}{c}\text { Agency } \\
\text { database }\end{array}$ & Station name & $\begin{array}{c}\text { USGS site } \\
\text { identification } \\
\text { number }\end{array}$ & $\begin{array}{l}\text { Local agency } \\
\text { unique site } \\
\text { identifier }\end{array}$ & County & $\begin{array}{l}\text { Latitude } \\
\text { (degree } \\
\text { minutes } \\
\text { and } \\
\text { seconds) } \\
\text { [DDMMSS] }\end{array}$ & $\begin{array}{l}\text { Longitude } \\
\text { (degree } \\
\text { minutes } \\
\text { and } \\
\text { seconds) } \\
\text { ] [DDMMSS] }\end{array}$ & $\begin{array}{c}\text { Date of } \\
\text { measure- } \\
\text { ment }\end{array}$ & $\begin{array}{c}\text { Water } \\
\text { level } \\
\text { altitude } \\
\text { (feet } \\
\text { above } \\
\text { NGVD 29) }\end{array}$ & Remarks \\
\hline FL & USGS-FLWSC & $\begin{array}{l}\text { ROMP CI-2 4" UFA near } \\
\text { Frostproof }\end{array}$ & 274522081303901 & - & Polk & 274523 & 813038 & $5 / 18 / 2010$ & 79 & \\
\hline FL & USGS-FLWSC & $\begin{array}{l}\text { ROMP CI-3 10" UFA near } \\
\text { Frostproof }\end{array}$ & 274545081342501 & - & Polk & 274546 & 813424 & $5 / 18 / 2010$ & 78 & \\
\hline FL & USGS-FLWSC & $\begin{array}{l}\text { ROMP DP WELL } 116 \text { NR } \\
\text { TSALA APOPKA }\end{array}$ & 285720082201301 & - & Citrus & 285721 & 822012 & $5 / 20 / 2010$ & 33 & \\
\hline FL & USGS-FLWSC & ROMP DV-1 4" UFA, Dover & 275926082123404 & - & Hillsborough & 275927 & 821233 & $5 / 18 / 2010$ & 54 & \\
\hline FL & USGS-FLWSC & $\begin{array}{l}\text { ROMP DV-2 6" UFA near } \\
\text { Dover }\end{array}$ & 275759082085402 & - & Hillsborough & 275800 & 820853 & $5 / 17 / 2010$ & 63 & \\
\hline FL & USGS-FLWSC & $\begin{array}{l}\text { ROMP TR 1-2 SUWANNEE } \\
\text { (11334 AND 229) }\end{array}$ & 265026081585401 & - & Charlotte & 265027 & 815853 & $5 / 17 / 2010$ & 40 & $\begin{array}{l}\text { Uncorrected hydrau- } \\
\text { lic head reported; } \\
\text { brackish to saline } \\
\text { water; not con- } \\
\text { toured on Potentio- } \\
\text { metric surface map }\end{array}$ \\
\hline FL & USGS-FLWSC & $\begin{array}{l}\text { ROMP TR 16-2 VAN BUREN- } \\
\text { SWNN }\end{array}$ & 281518082424301 & - & Pasco & 281519 & 824242 & $5 / 19 / 2010$ & 6 & \\
\hline FL & USGS-FLWSC & $\begin{array}{l}\text { ROMP TR 16-3 UFA Interface } \\
\text { near New Port Richey, FL }\end{array}$ & 281542082405601 & - & Pasco & 281542 & 824056 & $5 / 18 / 2010$ & 14 & \\
\hline FL & USGS-FLWSC & $\begin{array}{l}\text { ROMP TR 16-4 UP FLDN SWI } \\
\text { near near New Port Richey, } \\
\text { FL }\end{array}$ & 281526082395901 & - & Pasco & 281526 & 823958 & $5 / 18 / 2010$ & 17 & \\
\hline FL & USGS-FLWSC & $\begin{array}{l}\text { Romp TR 7-1 Tampa Well Nr } \\
\text { Bradenton }\end{array}$ & 272510082345701 & - & Manatee & 272511 & 823457 & $5 / 20 / 2010$ & 14 & \\
\hline FL & USGS-FLWSC & $\begin{array}{l}\text { ROMP TR 8-1-Suwannee UFA } \\
\text { at Rubonia }\end{array}$ & 273458082324705 & - & Manatee & 273459 & 823246 & $5 / 21 / 2010$ & 16 & \\
\hline FL & USGS-FLWSC & ROMP TR SA-1 4" UFA & 272049082324502 & - & Sarasota & 272050 & 823244 & $5 / 17 / 2010$ & 10 & \\
\hline
\end{tabular}


Table 1. Water-level measurements in Florida and parts of Georgia, South Carolina, and Alabama, May-June 2010.—Continued

[NGVD 29, National Geodetic Vertical Datum of 1929. Abbreviations for agency database: ACEPD, Alachua County Environmental Protection Department; FGS, Florida Geological Survey; GSA, Geological Survey of Alabama; NWFWMD, Northwest Florida Water Management District; SFWMD, South Florida Water Management District; SRWMD, Suwannee River Water Management District; USGSALWSC, U.S. Geological Survey Alabama Water Science Center; USGS-FLWSC, U.S. Geological Survey Florida Water Science Center; GAWSC, U.S. Geological Survey Georgia Water Science Center; SCWSC, U.S. Geological Survey South Carolina Water Science Center. Abbreviations for Station names: BLVD., boulevard; CO, county; CR, county road; E, east; HS, high school; IFAS, Institute of Food and Agricultural Services; LK, lake; MW, monitoring well; N, north; NE, northeast; NE, northeast; NF, national forest; NW, northwest; PK, park; R., river; RD., road; SE, southeast; SE, southeast; SR, state road; ST., street; TWR, tower; UFA, Upper Floridan aquifer; —, not available; \#, number; “, inches]

\begin{tabular}{|c|c|c|c|c|c|c|c|c|c|c|}
\hline State & $\begin{array}{c}\text { Agency } \\
\text { database }\end{array}$ & Station name & $\begin{array}{c}\text { USGS site } \\
\text { identification } \\
\text { number }\end{array}$ & $\begin{array}{l}\text { Local agency } \\
\text { unique site } \\
\text { identifier }\end{array}$ & County & $\begin{array}{l}\text { Latitude } \\
\text { (degree } \\
\text { minutes } \\
\text { and } \\
\text { seconds) } \\
\text { [DDMMSS] }\end{array}$ & $\begin{array}{l}\text { Longitude } \\
\text { (degree } \\
\text { minutes } \\
\text { and } \\
\text { seconds) } \\
\text { [DDMMSS] }\end{array}$ & $\begin{array}{c}\text { Date of } \\
\text { measure- } \\
\text { ment }\end{array}$ & $\begin{array}{c}\text { Water } \\
\text { level } \\
\text { altitude } \\
\text { (feet } \\
\text { above } \\
\text { NGVD 29) }\end{array}$ & Remarks \\
\hline FL & USGS-FLWSC & $\begin{array}{l}\text { Romp TR SA-3 FLRD Well at } \\
\text { Sarasota, FL }\end{array}$ & 272056082303701 & - & Sarasota & 272056 & 823037 & $5 / 17 / 2010$ & 16 & \\
\hline FL & USGS-FLWSC & $\begin{array}{l}\text { ROMP TR10-2 6" UFA near } \\
\text { Tampa }\end{array}$ & 275402082222701 & - & Hillsborough & 275403 & 822226 & $5 / 19 / 2010$ & 9 & \\
\hline FL & USGS-FLWSC & $\begin{array}{l}\text { ROMP TR11-2 6" UFA near } \\
\text { Tampa }\end{array}$ & 275705082222001 & - & Hillsborough & 275706 & 822219 & $5 / 19 / 2010$ & 15 & \\
\hline FL & USGS-FLWSC & $\begin{array}{l}\text { ROMP TR12-1 6" UFA SD } \\
\text { Replacement, Eisenhower } \\
\text { Blvd, Tampa }\end{array}$ & 275820082324602 & - & Hillsborough & 275818 & 823249 & $5 / 20 / 2010$ & 4 & \\
\hline FL & USGS-FLWSC & $\begin{array}{l}\text { ROMP TR13-3 6" UFA near } \\
\text { Citrus Park }\end{array}$ & 280354082381901 & - & Hillsborough & 280355 & 823818 & $5 / 19 / 2010$ & 15 & \\
\hline FL & USGS-FLWSC & $\begin{array}{l}\text { ROMP TR14-1 10" UFA, } \\
\text { Safety Harbor }\end{array}$ & 280002082412602 & - & Pinellas & 280003 & 824125 & $5 / 18 / 2010$ & 8 & \\
\hline FL & USGS-FLWSC & ROMP TR14-2 4" UFA & 280132082452801 & - & Pinellas & 280133 & 824527 & $5 / 18 / 2010$ & 2 & \\
\hline FL & USGS-FLWSC & $\begin{array}{l}\text { ROMP TR14-3 10" UFA near } \\
\text { Dunedin }\end{array}$ & 280118082434502 & - & Pinellas & 280119 & 824344 & $5 / 18 / 2010$ & 7 & \\
\hline FL & USGS-FLWSC & $\begin{array}{l}\text { ROMP TR15-1 6" UFA near } \\
\text { Tarpon Springs }\end{array}$ & 280753082465201 & - & Pinellas & 280754 & 824651 & $5 / 19 / 2010$ & 3 & \\
\hline FL & USGS-FLWSC & $\begin{array}{l}\text { ROMP TR15-2 6" UFA near } \\
\text { Tarpon Springs }\end{array}$ & 280747082452001 & - & Pinellas & 280748 & 824519 & $5 / 19 / 2010$ & 3 & \\
\hline FL & USGS-FLWSC & $\begin{array}{l}\text { ROMP TR15-3 6" UFA near } \\
\text { Tarpon Springs }\end{array}$ & 280734082442101 & - & Pinellas & 280735 & 824420 & $5 / 20 / 2010$ & 5 & \\
\hline FL & USGS-FLWSC & $\begin{array}{l}\text { ROMP TR17-1 6" UFA, Bayo- } \\
\text { net Point }\end{array}$ & 281917082420901 & - & Pasco & 281918 & 824208 & $5 / 19 / 2010$ & 4 & \\
\hline FL & USGS-FLWSC & $\begin{array}{l}\text { ROMP TR17-3 6" UFA near } \\
\text { Bayonet Point }\end{array}$ & 281922082403901 & - & Pasco & 281923 & 824038 & $5 / 18 / 2010$ & 3 & \\
\hline
\end{tabular}


Table 1. Water-level measurements in Florida and parts of Georgia, South Carolina, and Alabama, May-June 2010.-Continued

[NGVD 29, National Geodetic Vertical Datum of 1929. Abbreviations for agency database: ACEPD, Alachua County Environmental Protection Department; FGS, Florida Geological Survey; GSA, Geological Survey of Alabama; NWFWMD, Northwest Florida Water Management District; SFWMD, South Florida Water Management District; SRWMD, Suwannee River Water Management District; USGSALWSC, U.S. Geological Survey Alabama Water Science Center; USGS-FLWSC, U.S. Geological Survey Florida Water Science Center; GAWSC, U.S. Geological Survey Georgia Water Science Center; SCWSC, U.S. Geological Survey South Carolina Water Science Center. Abbreviations for Station names: BLVD., boulevard; CO, county; CR, county road; E, east; HS, high school; IFAS, Institute of Food and Agricultural Services; LK, lake; MW, monitoring well; N, north; NE, northeast; NE, northeast; NF, national forest; NW, northwest; PK, park; R., river; RD., road; SE, southeast; SE, southeast; SR, state road; ST., street; TWR, tower; UFA, Upper Floridan aquifer; - - not available; \#, number; “, inches]

\begin{tabular}{|c|c|c|c|c|c|c|c|c|c|c|}
\hline State & $\begin{array}{c}\text { Agency } \\
\text { database }\end{array}$ & Station name & $\begin{array}{c}\text { USGS site } \\
\text { identification } \\
\text { number }\end{array}$ & $\begin{array}{l}\text { Local agency } \\
\text { unique site } \\
\text { identifier }\end{array}$ & County & $\begin{array}{l}\text { Latitude } \\
\text { (degree } \\
\text { minutes } \\
\text { and } \\
\text { seconds) } \\
\text { [DDMMSS] }\end{array}$ & $\begin{array}{l}\text { Longitude } \\
\text { (degree } \\
\text { minutes } \\
\text { and } \\
\text { seconds) } \\
\text { ] [DDMMSS] }\end{array}$ & $\begin{array}{c}\text { Date of } \\
\text { measure- } \\
\text { ment }\end{array}$ & $\begin{array}{c}\text { Water } \\
\text { level } \\
\text { altitude } \\
\text { (feet } \\
\text { above } \\
\text { NGVD 29) }\end{array}$ & Remarks \\
\hline FL & USGS-FLWSC & $\begin{array}{l}\text { ROMP TR18-1 6" UFA near } \\
\text { Aripeka }\end{array}$ & 282742082375901 & - & Hernando & 282743 & 823758 & $5 / 17 / 2010$ & 14 & \\
\hline FL & USGS-FLWSC & $\begin{array}{l}\text { ROMP TR18-3 16" UFA near } \\
\text { Aripeka }\end{array}$ & 282613082381701 & - & Hernando & 282614 & 823816 & $5 / 17 / 2010$ & 12 & \\
\hline FL & USGS-FLWSC & $\begin{array}{l}\text { ROMP TR19-2 (Whitehurst) 6" } \\
\text { UFA near Bayport }\end{array}$ & 283243082365701 & - & Hernando & 283244 & 823656 & $5 / 17 / 2010$ & 6 & \\
\hline FL & USGS-FLWSC & $\begin{array}{l}\text { ROMP TR21-2 6" UFA near } \\
\text { Homosassa Springs }\end{array}$ & 285112082354401 & - & Citrus & 285113 & 823543 & $5 / 18 / 2010$ & 2 & \\
\hline FL & USGS-FLWSC & $\begin{array}{l}\text { ROMP TR21-3 6" UFA near } \\
\text { Crystal River }\end{array}$ & 285234082341901 & - & Citrus & 285235 & 823418 & $5 / 18 / 2010$ & 3 & \\
\hline FL & USGS-FLWSC & $\begin{array}{l}\text { ROMP TR3-1 4" UFA near El } \\
\text { Jobean }\end{array}$ & 265638082130706 & - & Charlotte & 265639 & 821306 & $5 / 17 / 2010$ & 28 & $\begin{array}{l}\text { Uncorrected hydrau- } \\
\text { lic head reported; } \\
\text { brackish to saline } \\
\text { water; not con- } \\
\text { toured on Potentio- } \\
\text { metric surface map }\end{array}$ \\
\hline FL & USGS-FLWSC & $\begin{array}{l}\text { ROMP TR3-3 6" UFA near } \\
\text { Englewood }\end{array}$ & 265531082194803 & - & Charlotte & 265532 & 821947 & $5 / 17 / 2010$ & 15 & $\begin{array}{l}\text { Uncorrected hydrau- } \\
\text { lic head reported; } \\
\text { brackish to saline } \\
\text { water; not con- } \\
\text { toured on Potentio- } \\
\text { metric surface map }\end{array}$ \\
\hline FL & USGS-FLWSC & $\begin{array}{l}\text { ROMP TR4-2 6" UFA near } \\
\text { Venice }\end{array}$ & 270240082235701 & - & Sarasota & 270241 & 822356 & $5 / 21 / 2010$ & 22 & $\begin{array}{l}\text { Uncorrected hydrau- } \\
\text { lic head reported; } \\
\text { brackish to saline } \\
\text { water; not con- } \\
\text { toured on Potentio- } \\
\text { metric surface map }\end{array}$ \\
\hline
\end{tabular}


Table 1. Water-level measurements in Florida and parts of Georgia, South Carolina, and Alabama, May-June 2010.—Continued

[NGVD 29, National Geodetic Vertical Datum of 1929. Abbreviations for agency database: ACEPD, Alachua County Environmental Protection Department; FGS, Florida Geological Survey; GSA, Geological Survey of Alabama; NWFWMD, Northwest Florida Water Management District; SFWMD, South Florida Water Management District; SRWMD, Suwannee River Water Management District; USGSALWSC, U.S. Geological Survey Alabama Water Science Center; USGS-FLWSC, U.S. Geological Survey Florida Water Science Center; GAWSC, U.S. Geological Survey Georgia Water Science Center; SCWSC, U.S. Geological Survey South Carolina Water Science Center. Abbreviations for Station names: BLVD., boulevard; CO, county; CR, county road; E, east; HS, high school; IFAS, Institute of Food and Agricultural Services; LK, lake; MW, monitoring well; N, north; NE, northeast; NE, northeast; NF, national forest; NW, northwest; PK, park; R., river; RD., road; SE, southeast; SE, southeast; SR, state road; ST., street; TWR, tower; UFA, Upper Floridan aquifer; —, not available; \#, number; “, inches]

\begin{tabular}{|c|c|c|c|c|c|c|c|c|c|c|}
\hline State & $\begin{array}{l}\text { Agency } \\
\text { database }\end{array}$ & Station name & $\begin{array}{l}\text { USGS site } \\
\text { identification } \\
\text { number }\end{array}$ & $\begin{array}{l}\text { Local agency } \\
\text { unique site } \\
\text { identifier }\end{array}$ & County & $\begin{array}{l}\text { Latitude } \\
\text { (degree } \\
\text { minutes } \\
\text { and } \\
\text { seconds) } \\
\text { [DDMMSS] }\end{array}$ & $\begin{array}{l}\text { Longitude } \\
\text { (degree } \\
\text { minutes } \\
\text { and } \\
\text { seconds) } \\
\text { ] [DDMMSS] }\end{array}$ & $\begin{array}{c}\text { Date of } \\
\text { measure- } \\
\text { ment }\end{array}$ & $\begin{array}{l}\text { Water } \\
\text { level } \\
\text { altitude } \\
\text { (feet } \\
\text { above } \\
\text { NGVD 29) }\end{array}$ & Remarks \\
\hline FL & USGS-FLWSC & ROMP TR5-1 4" UFA, Laurel & 270808082270502 & - & Sarasota & 270809 & 822704 & $5 / 19 / 2010$ & 18 & $\begin{array}{l}\text { Uncorrected hydrau- } \\
\text { lic head reported; } \\
\text { brackish to saline } \\
\text { water; not con- } \\
\text { toured on Potentio- } \\
\text { metric surface map }\end{array}$ \\
\hline FL & USGS-FLWSC & $\begin{array}{l}\text { ROMP TR5-2 6" UFA near } \\
\text { Laurel }\end{array}$ & 270919082234205 & - & Sarasota & 270920 & 822341 & $5 / 20 / 2010$ & 23 & $\begin{array}{l}\text { Uncorrected hydrau- } \\
\text { lic head reported; } \\
\text { brackish to saline } \\
\text { water; not con- } \\
\text { toured on Potentio- } \\
\text { metric surface map }\end{array}$ \\
\hline FL & USGS-FLWSC & $\begin{array}{l}\text { ROMP TR7-2 6" UFA near } \\
\text { Oneco }\end{array}$ & 272612082330101 & - & Manatee & 272613 & 823300 & $5 / 20 / 2010$ & 15 & \\
\hline FL & USGS-FLWSC & $\begin{array}{l}\text { ROMP TR7-4 6" UFA near } \\
\text { Bradenton }\end{array}$ & 272539082292002 & - & Manatee & 272540 & 822919 & $5 / 19 / 2010$ & 9 & \\
\hline FL & USGS-FLWSC & $\begin{array}{l}\text { ROMP TR9-1 8" UFA near } \\
\text { Ruskin }\end{array}$ & 274421082275401 & - & Hillsborough & 274422 & 822753 & $5 / 20 / 2010$ & 12 & \\
\hline FL & USGS-FLWSC & $\begin{array}{l}\text { ROMP TR9-2 6" UFA, Apollo } \\
\text { Beach }\end{array}$ & 274554082233801 & - & Hillsborough & 274555 & 822337 & $5 / 20 / 2010$ & 3 & \\
\hline FL & USGS-FLWSC & $\begin{array}{l}\text { ROMP TR9-3 8" UFA near } \\
\text { Ruskin }\end{array}$ & 274428082251503 & - & Hillsborough & 274429 & 822514 & $5 / 20 / 2010$ & -5 & \\
\hline FL & USGS-FLWSC & $\begin{array}{l}\text { ROSE CREEK TOWER-JOHN } \\
\text { FOLKS-DOF 4" UFA NR } \\
\text { LK CITY }\end{array}$ & 300612082363101 & -041734002 & Columbia & 300613 & 823630 & $5 / 18 / 2010$ & 38 & $\begin{array}{l}\text { SRWMD Site iden- } \\
\text { tification number } \\
-041734002\end{array}$ \\
\hline FL & USGS-FLWSC & $\begin{array}{l}\text { ROSSINI UFA WEST OF } \\
\text { TRILBY }\end{array}$ & 282717082142001 & - & Pasco & 282718 & 821419 & $5 / 17 / 2010$ & 54 & \\
\hline FL & USGS-FLWSC & RUSSANO AT LAKE LYND- & 283804082211001 & - & Hernando & 283805 & 822205 & $5 / 18 / 2010$ & 22 & \\
\hline
\end{tabular}


Table 1. Water-level measurements in Florida and parts of Georgia, South Carolina, and Alabama, May-June 2010.—Continued

[NGVD 29, National Geodetic Vertical Datum of 1929. Abbreviations for agency database: ACEPD, Alachua County Environmental Protection Department; FGS, Florida Geological Survey; GSA, Geological Survey of Alabama; NWFWMD, Northwest Florida Water Management District; SFWMD, South Florida Water Management District; SRWMD, Suwannee River Water Management District; USGSALWSC, U.S. Geological Survey Alabama Water Science Center; USGS-FLWSC, U.S. Geological Survey Florida Water Science Center; GAWSC, U.S. Geological Survey Georgia Water Science Center; SCWSC, U.S. Geological Survey South Carolina Water Science Center. Abbreviations for Station names: BLVD., boulevard; CO, county; CR, county road; E, east; HS, high school; IFAS, Institute of Food and Agricultural Services; LK, lake; MW, monitoring well; N, north; NE, northeast; NE, northeast; NF, national forest; NW, northwest; PK, park; R., river; RD., road; SE, southeast; SE, southeast; SR, state road; ST., street; TWR, tower; UFA, Upper Floridan aquifer; - - not available; \#, number; “, inches]

\begin{tabular}{|c|c|c|c|c|c|c|c|c|c|c|}
\hline State & $\begin{array}{l}\text { Agency } \\
\text { database }\end{array}$ & Station name & $\begin{array}{c}\text { USGS site } \\
\text { identification } \\
\text { number }\end{array}$ & $\begin{array}{l}\text { Local agency } \\
\text { unique site } \\
\text { identifier }\end{array}$ & County & $\begin{array}{c}\text { Latitude } \\
\text { (degree } \\
\text { minutes } \\
\text { and } \\
\text { seconds) } \\
\text { [DDMMSS] }\end{array}$ & $\begin{array}{l}\text { Longitude } \\
\text { (degree } \\
\text { minutes } \\
\text { and } \\
\text { seconds) } \\
\text { ] [DDMMSS] }\end{array}$ & $\begin{array}{c}\text { Date of } \\
\text { measure- } \\
\text { ment }\end{array}$ & $\begin{array}{c}\text { Water } \\
\text { level } \\
\text { altitude } \\
\text { (feet } \\
\text { above } \\
\text { NGVD 29) }\end{array}$ & Remarks \\
\hline FL & USGS-FLWSC & S-0001 GENEVA WELL UFA & 284247081070801 & - & Seminole & 284248 & 810707 & $5 / 20 / 2010$ & 21 & \\
\hline FL & USGS-FLWSC & $\begin{array}{l}\text { S-0025 KILBEE \#3 TEST 4" } \\
\text { UFA NR GENEVA }\end{array}$ & 284217081023001 & - & Seminole & 284218 & 810229 & $5 / 20 / 2010$ & 9 & \\
\hline FL & USGS-FLWSC & $\begin{array}{l}\text { S-0086 OSCEOLA LANDFILL } \\
\text { 6" UFA }\end{array}$ & 284715081051802 & - & Seminole & 284716 & 810517 & $5 / 20 / 2010$ & 13 & \\
\hline FL & USGS-FLWSC & S-0125 6" UFA, Longwood & 284147081220201 & - & Seminole & 284148 & 812201 & $5 / 20 / 2010$ & 38 & \\
\hline FL & USGS-FLWSC & $\begin{array}{l}\text { S-0224 UFA THE FOREST } \\
\text { 845-120-05 }\end{array}$ & 284533081204801 & - & Seminole & 284534 & 812047 & $5 / 20 / 2010$ & 34 & \\
\hline FL & USGS-FLWSC & $\begin{array}{l}\text { S-1014 CHARLOTTE ST } 6 " \\
\text { UFA }\end{array}$ & 284052081212601 & - & Seminole & 284053 & 812125 & $5 / 20 / 2010$ & 45 & \\
\hline FL & USGS-FLWSC & $\begin{array}{l}\text { S-1230 YANKEE LAKE STP } \\
\text { UFA }\end{array}$ & 284923081234802 & - & Seminole & 284924 & 812347 & $5 / 20 / 2010$ & 21 & \\
\hline FL & USGS-FLWSC & $\begin{array}{l}\text { S-1253 (OLD) GENEVA FIRE } \\
\text { STATION 6" UFA }\end{array}$ & 284412081071102 & - & Seminole & 284413 & 810710 & $5 / 20 / 2010$ & 17 & \\
\hline FL & USGS-FLWSC & $\begin{array}{l}\text { Sanlon Ranch 24" UFA near } \\
\text { Eaton Park }\end{array}$ & 275959081552501 & - & Polk & 280000 & 815524 & $5 / 20 / 2010$ & 93 & \\
\hline FL & USGS-FLWSC & $\begin{array}{l}\text { Sarasota 8" UFA \#9 UFA near } \\
\quad \text { Sarasota }\end{array}$ & 271938082251801 & - & Sarasota & 271939 & 822517 & $5 / 19 / 2010$ & 14 & \\
\hline FL & USGS-FLWSC & SCE 116 RAINBOW ACRES & 290752082271101 & - & Marion & 290753 & 822710 & $5 / 24 / 2010$ & 35 & \\
\hline FL & USGS-FLWSC & $\begin{array}{l}\text { SCE } 118 \text { LAKE TROPICANA } \\
\text { RANCHETTE }\end{array}$ & 290913082245601 & - & Marion & 290914 & 822455 & $5 / 24 / 2010$ & 37 & \\
\hline FL & USGS-FLWSC & $\begin{array}{l}\text { SCE } 158 \text { LITTLE LAKE BON- } \\
\text { ABLE WELL }\end{array}$ & 290910082315001 & - & Marion & 290911 & 823149 & $5 / 24 / 2010$ & 43 & \\
\hline FL & USGS-FLWSC & $\begin{array}{l}\text { SEA WORLD DRIVE WELL } \\
\text { REPLACEMENT NR VINE- } \\
\text { LAND }\end{array}$ & 282434081283102 & - & Orange & 282435 & 812830 & $5 / 17 / 2010$ & 57 & \\
\hline
\end{tabular}


Table 1. Water-level measurements in Florida and parts of Georgia, South Carolina, and Alabama, May-June 2010.—Continued

[NGVD 29, National Geodetic Vertical Datum of 1929. Abbreviations for agency database: ACEPD, Alachua County Environmental Protection Department; FGS, Florida Geological Survey; GSA, Geological Survey of Alabama; NWFWMD, Northwest Florida Water Management District; SFWMD, South Florida Water Management District; SRWMD, Suwannee River Water Management District; USGSALWSC, U.S. Geological Survey Alabama Water Science Center; USGS-FLWSC, U.S. Geological Survey Florida Water Science Center; GAWSC, U.S. Geological Survey Georgia Water Science Center; SCWSC, U.S. Geological Survey South Carolina Water Science Center. Abbreviations for Station names: BLVD., boulevard; CO, county; CR, county road; E, east; HS, high school; IFAS, Institute of Food and Agricultural Services; LK, lake; MW, monitoring well; N, north; NE, northeast; NE, northeast; NF, national forest; NW, northwest; PK, park; R., river; RD., road; SE, southeast; SE, southeast; SR, state road; ST., street; TWR, tower; UFA, Upper Floridan aquifer; - , not available; \#, number; “, inches]

\begin{tabular}{|c|c|c|c|c|c|c|c|c|c|c|}
\hline State & $\begin{array}{l}\text { Agency } \\
\text { database }\end{array}$ & Station name & $\begin{array}{c}\text { USGS site } \\
\text { identification } \\
\text { number }\end{array}$ & $\begin{array}{l}\text { Local agency } \\
\text { unique site } \\
\text { identifier }\end{array}$ & County & $\begin{array}{c}\text { Latitude } \\
\text { (degree } \\
\text { minutes } \\
\text { and } \\
\text { seconds) } \\
\text { [DDMMSS] }\end{array}$ & $\begin{array}{l}\text { Longitude } \\
\text { (degree } \\
\text { minutes } \\
\text { and } \\
\text { seconds) } \\
\text { [DDMMSS] }\end{array}$ & $\begin{array}{l}\text { Date of } \\
\text { measure- } \\
\text { ment }\end{array}$ & $\begin{array}{c}\text { Water } \\
\text { level } \\
\text { altitude } \\
\text { (feet } \\
\text { above } \\
\text { NGVD 29) }\end{array}$ & Remarks \\
\hline FL & USGS-FLWSC & $\begin{array}{l}\text { Sec } 21 \text { Goodwin 2" UFA near } \\
\text { Lutz }\end{array}$ & 280734082313301 & - & Hillsborough & 280735 & 823132 & $5 / 19 / 2010$ & 44 & \\
\hline $\mathrm{FL}$ & USGS-FLWSC & $\begin{array}{l}\text { Sec 21-13 Hillsborough 12" } \\
\text { UFA near Lutz }\end{array}$ & 280702082302801 & - & Hillsborough & 280703 & 823027 & $5 / 19 / 2010$ & 44 & \\
\hline FL & USGS-FLWSC & $\begin{array}{l}\text { Serw-D 6" UFA near Masaryk- } \\
\text { town }\end{array}$ & 282035082283701 & - & Pasco & 282036 & 822836 & $5 / 17 / 2010$ & 67 & \\
\hline FL & USGS-FLWSC & $\begin{array}{l}\text { Serw-D 6" UFA near Masaryk- } \\
\text { town }\end{array}$ & 282202082270801 & - & Pasco & 282203 & 822707 & $5 / 18 / 2010$ & 58 & \\
\hline FL & USGS-FLWSC & $\begin{array}{l}\text { Seven Springs FLRD Well near } \\
\text { New Port Richey, FL }\end{array}$ & 281223082404801 & - & Pasco & 281223 & 824049 & $5 / 18 / 2010$ & 18 & \\
\hline FL & USGS-FLWSC & $\begin{array}{l}\text { Sheldon Rd 6" UFA near Town } \\
\text { N' Country }\end{array}$ & 280053082350202 & - & Hillsborough & 280054 & 823501 & $6 / 1 / 2010$ & 7 & \\
\hline FL & USGS-FLWSC & $\begin{array}{l}\text { SHINGLE CREEK 4" UFA, } \\
\text { SR 531A }\end{array}$ & 281559081260701 & - & Osceola & 281600 & 812606 & $5 / 17 / 2010$ & 56 & \\
\hline FL & USGS-FLWSC & $\begin{array}{l}\text { SJ0005 G. Oesterreicher 6" } \\
\text { UFA Artesian near Palm } \\
\text { Valley }\end{array}$ & 300758081230501 & - & St Johns & 300759 & 812304 & $5 / 26 / 2010$ & 27 & \\
\hline FL & USGS-FLWSC & $\begin{array}{l}\text { SJ0027 BAKERSVILLE TWR } \\
\text { (SJ0161) UFA }\end{array}$ & 295427081293101 & - & St Johns & 295428 & 812930 & $5 / 26 / 2010$ & 28 & \\
\hline FL & USGS-FLWSC & $\begin{array}{l}\text { SJ0029 DURBIN FIRETOW- } \\
\text { ER UFA (SJ0163) }\end{array}$ & 300507081272701 & - & St Johns & 300508 & 812726 & $5 / 26 / 2010$ & 37 & \\
\hline FL & USGS-FLWSC & $\begin{array}{l}\text { SJ0092 SJ CO PARKS/REC } \\
\text { OFFICE ANASTASIA WTP } \\
\text { UFA }\end{array}$ & 295132081164801 & - & St Johns & 295133 & 811647 & $5 / 26 / 2010$ & 16 & \\
\hline FL & USGS-FLWSC & $\begin{array}{l}\text { SJ0099 (SJ0060) DEE DOT } \\
\text { RANCH UFA CRACKER } \\
\text { LODGE }\end{array}$ & 301408081253101 & - & St Johns & 301409 & 812530 & $5 / 26 / 2010$ & 19 & \\
\hline
\end{tabular}


Table 1. Water-level measurements in Florida and parts of Georgia, South Carolina, and Alabama, May-June 2010.—Continued

[NGVD 29, National Geodetic Vertical Datum of 1929. Abbreviations for agency database: ACEPD, Alachua County Environmental Protection Department; FGS, Florida Geological Survey; GSA, Geological Survey of Alabama; NWFWMD, Northwest Florida Water Management District; SFWMD, South Florida Water Management District; SRWMD, Suwannee River Water Management District; USGSALWSC, U.S. Geological Survey Alabama Water Science Center; USGS-FLWSC, U.S. Geological Survey Florida Water Science Center; GAWSC, U.S. Geological Survey Georgia Water Science Center; SCWSC, U.S. Geological Survey South Carolina Water Science Center. Abbreviations for Station names: BLVD., boulevard; CO, county; CR, county road; E, east; HS, high school; IFAS, Institute of Food and Agricultural Services; LK, lake; MW, monitoring well; N, north; NE, northeast; NE, northeast; NF, national forest; NW, northwest; PK, park; R., river; RD., road; SE, southeast; SE, southeast; SR, state road; ST., street; TWR, tower; UFA, Upper Floridan aquifer; - - not available; \#, number; “, inches]

\begin{tabular}{|c|c|c|c|c|c|c|c|c|c|c|}
\hline State & $\begin{array}{l}\text { Agency } \\
\text { database }\end{array}$ & Station name & $\begin{array}{c}\text { USGS site } \\
\text { identification } \\
\text { number }\end{array}$ & $\begin{array}{l}\text { Local agency } \\
\text { unique site } \\
\text { identifier }\end{array}$ & County & $\begin{array}{l}\text { Latitude } \\
\text { (degree } \\
\text { minutes } \\
\text { and } \\
\text { seconds) } \\
\text { [DDMMSS] }\end{array}$ & $\begin{array}{l}\text { Longitude } \\
\text { (degree } \\
\text { minutes } \\
\text { and } \\
\text { seconds) } \\
\text { ] [DDMMSS] }\end{array}$ & $\begin{array}{l}\text { Date of } \\
\text { measure- } \\
\text { ment }\end{array}$ & $\begin{array}{c}\text { Water } \\
\text { level } \\
\text { altitude } \\
\text { (feet } \\
\text { above } \\
\text { NGVD 29) }\end{array}$ & Remarks \\
\hline FL & USGS-FLWSC & $\begin{array}{l}\text { SJ0102 (SJ-63) DEE DOT } \\
\text { RANCH UFA, BULL PAS- } \\
\text { TURE }\end{array}$ & 301212081252401 & - & St Johns & 301213 & 812523 & $5 / 26 / 2010$ & 40 & \\
\hline FL & USGS-FLWSC & $\begin{array}{l}\text { SJ0115 (SJ0104) } 33 \text { DOT } \\
\text { MEADOWBRICK UFA }\end{array}$ & 293729081221201 & - & Flagler & 293730 & 812211 & $5 / 26 / 2010$ & 16 & \\
\hline FL & USGS-FLWSC & $\begin{array}{l}\text { SJ0263 D. REID 6" } \\
\text { UFA;USGS SJ-105 }\end{array}$ & 294128081291301 & - & St Johns & 294129 & 812912 & $5 / 26 / 2010$ & 14 & \\
\hline FL & USGS-FLWSC & SJ0317 SIKES UFA & 294701081263301 & - & St Johns & 294702 & 812632 & $5 / 26 / 2010$ & 21 & \\
\hline FL & USGS-FLWSC & SJ0324 TROUT CREEK PARK & 295903081333901 & - & St Johns & 295903 & 813339 & $5 / 26 / 2010$ & 27 & \\
\hline FL & USGS-FLWSC & SJ-0331 WOODLAWN RD & 295604081223503 & - & St Johns & 295605 & 812234 & $5 / 26 / 2010$ & 28 & \\
\hline FL & USGS-FLWSC & $\begin{array}{l}\text { SJ0508 Greenbrier Rd Middle } \\
\text { School 12" UFA }\end{array}$ & 300340081383901 & - & St Johns & 300341 & 813838 & $5 / 26 / 2010$ & 31 & \\
\hline FL & USGS-FLWSC & SJ0516 DUPONT CTR UFA & 294519081184502 & - & St Johns & 294520 & 811844 & $5 / 26 / 2010$ & 17 & \\
\hline FL & USGS-FLWSC & SJ0602 DOT UFA I95 SOUTH & 294213081194401 & - & St Johns & 294214 & 811943 & $5 / 26 / 2010$ & 17 & \\
\hline FL & USGS-FLWSC & SJ0824 Treaty Park 6" UFA & 295000081212702 & - & St Johns & 295001 & 812126 & $5 / 26 / 2010$ & 24 & \\
\hline FL & USGS-FLWSC & $\begin{array}{l}\text { SJRWMD C-0059 JOHN } \\
\text { HUNTLEY UFA (C-0005) }\end{array}$ & 300649081485901 & - & Clay & 300650 & 814858 & $5 / 20 / 2010$ & 39 & \\
\hline FL & USGS-FLWSC & SLF-3 ROY HAMILTON & 272927080261601 & - & St Lucie & 272928 & 802615 & $5 / 19 / 2010$ & 38 & $\begin{array}{l}\text { Uncorrected hydrau- } \\
\text { lic head reported; } \\
\text { brackish to saline } \\
\text { water; not con- } \\
\text { toured on Potentio- } \\
\text { metric surface map }\end{array}$ \\
\hline
\end{tabular}


Table 1. Water-level measurements in Florida and parts of Georgia, South Carolina, and Alabama, May-June 2010.—Continued

[NGVD 29, National Geodetic Vertical Datum of 1929. Abbreviations for agency database: ACEPD, Alachua County Environmental Protection Department; FGS, Florida Geological Survey; GSA, Geological Survey of Alabama; NWFWMD, Northwest Florida Water Management District; SFWMD, South Florida Water Management District; SRWMD, Suwannee River Water Management District; USGSALWSC, U.S. Geological Survey Alabama Water Science Center; USGS-FLWSC, U.S. Geological Survey Florida Water Science Center; GAWSC, U.S. Geological Survey Georgia Water Science Center; SCWSC, U.S. Geological Survey South Carolina Water Science Center. Abbreviations for Station names: BLVD., boulevard; CO, county; CR, county road; E, east; HS, high school; IFAS, Institute of Food and Agricultural Services; LK, lake; MW, monitoring well; N, north; NE, northeast; NE, northeast; NF, national forest; NW, northwest; PK, park; R., river; RD., road; SE, southeast; SE, southeast; SR, state road; ST., street; TWR, tower; UFA, Upper Floridan aquifer; - , not available; \#, number; “, inches]

\begin{tabular}{|c|c|c|c|c|c|c|c|c|c|c|}
\hline State & $\begin{array}{c}\text { Agency } \\
\text { database }\end{array}$ & Station name & $\begin{array}{c}\text { USGS site } \\
\text { identification } \\
\text { number }\end{array}$ & $\begin{array}{l}\text { Local agency } \\
\text { unique site } \\
\text { identifier }\end{array}$ & County & $\begin{array}{l}\text { Latitude } \\
\text { (degree } \\
\text { minutes } \\
\text { and } \\
\text { seconds) } \\
\text { [DDMMSS] }\end{array}$ & $\begin{array}{l}\text { Longitude } \\
\text { (degree } \\
\text { minutes } \\
\text { and } \\
\text { seconds) } \\
\text { [DDMMSS] }\end{array}$ & $\begin{array}{c}\text { Date of } \\
\text { measure- } \\
\text { ment }\end{array}$ & $\begin{array}{c}\text { Water } \\
\text { level } \\
\text { altitude } \\
\text { (feet } \\
\text { above } \\
\text { NGVD 29) }\end{array}$ & Remarks \\
\hline FL & USGS-FLWSC & SLF-36 & 272840080315601 & - & St Lucie & 272841 & 803155 & $5 / 19 / 2010$ & 39 & $\begin{array}{l}\text { Uncorrected hydrau- } \\
\text { lic head reported; } \\
\text { brackish to saline } \\
\text { water; not con- } \\
\text { toured on Potentio- } \\
\text { metric surface map }\end{array}$ \\
\hline FL & USGS-FLWSC & SLF-46 & 273007080182501 & - & St Lucie & 273008 & 801824 & $5 / 19 / 2010$ & 34 & $\begin{array}{l}\text { Uncorrected hydrau- } \\
\text { lic head reported; } \\
\text { brackish to saline } \\
\text { water; not con- } \\
\text { toured on Potentio- } \\
\text { metric surface map }\end{array}$ \\
\hline FL & USGS-FLWSC & SLF-9 ALTO ADAMS JR. & 272650080265001 & - & St Lucie & 272651 & 803527 & $5 / 19 / 2010$ & 38 & $\begin{array}{l}\text { Uncorrected hydrau- } \\
\text { lic head reported; } \\
\text { brackish to saline } \\
\text { water; not con- } \\
\text { toured on Potentio- } \\
\text { metric surface map }\end{array}$ \\
\hline FL & USGS-FLWSC & $\begin{array}{l}\text { SMITH \#2 UFA NR CHERRY } \\
\text { LAKE }\end{array}$ & 285420081571901 & - & Sumter & 285421 & 815718 & $5 / 19 / 2010$ & 53 & \\
\hline FL & USGS-FLWSC & $\begin{array}{l}\text { SMITH BROTHERS NR } \\
\text { RALEIGH }\end{array}$ & 292816082234501 & - & Marion & 292817 & 822344 & $5 / 25 / 2010$ & 53 & \\
\hline FL & USGS-FLWSC & Sorrells Bros UFA Nr Arcadia & 271720081521501 & - & De Soto & 271721 & 815214 & $5 / 20 / 2010$ & 39 & \\
\hline FL & USGS-FLWSC & $\begin{array}{l}\text { South Pasco West near Odessa, } \\
\text { FL }\end{array}$ & 281112082315501 & - & Pasco & 281112 & 823155 & $5 / 18 / 2010$ & 48 & \\
\hline FL & USGS-FLWSC & $\begin{array}{l}\text { South Pine Lakes 4" UFA, SR } \\
\quad 44\end{array}$ & 285539081262901 & - & Lake & 285540 & 812628 & $5 / 26 / 2010$ & 37 & \\
\hline FL & USGS-FLWSC & $\begin{array}{l}\text { SPESSARD HOLLAND PARK } \\
\text { SOUTH }\end{array}$ & 280256080325601 & - & Brevard & 280257 & 803255 & $5 / 25 / 2010$ & 30 & \\
\hline
\end{tabular}


Table 1. Water-level measurements in Florida and parts of Georgia, South Carolina, and Alabama, May-June 2010.-Continued

[NGVD 29, National Geodetic Vertical Datum of 1929. Abbreviations for agency database: ACEPD, Alachua County Environmental Protection Department; FGS, Florida Geological Survey; GSA, Geological Survey of Alabama; NWFWMD, Northwest Florida Water Management District; SFWMD, South Florida Water Management District; SRWMD, Suwannee River Water Management District; USGSALWSC, U.S. Geological Survey Alabama Water Science Center; USGS-FLWSC, U.S. Geological Survey Florida Water Science Center; GAWSC, U.S. Geological Survey Georgia Water Science Center; SCWSC, U.S. Geological Survey South Carolina Water Science Center. Abbreviations for Station names: BLVD., boulevard; CO, county; CR, county road; E, east; HS, high school; IFAS, Institute of Food and Agricultural Services; LK, lake; MW, monitoring well; N, north; NE, northeast; NE, northeast; NF, national forest; NW, northwest; PK, park; R., river; RD., road; SE, southeast; SE, southeast; SR, state road; ST., street; TWR, tower; UFA, Upper Floridan aquifer; - - not available; \#, number; “, inches]

\begin{tabular}{|c|c|c|c|c|c|c|c|c|c|c|}
\hline State & $\begin{array}{c}\text { Agency } \\
\text { database }\end{array}$ & Station name & $\begin{array}{c}\text { USGS site } \\
\text { identification } \\
\text { number }\end{array}$ & $\begin{array}{l}\text { Local agency } \\
\text { unique site } \\
\text { identifier }\end{array}$ & County & $\begin{array}{l}\text { Latitude } \\
\text { (degree } \\
\text { minutes } \\
\text { and } \\
\text { seconds) } \\
\text { [DDMMSS] }\end{array}$ & $\begin{array}{l}\text { Longitude } \\
\text { (degree } \\
\text { minutes } \\
\text { and } \\
\text { seconds) } \\
\text { [DDMMSS] }\end{array}$ & $\begin{array}{c}\text { Date of } \\
\text { measure- } \\
\text { ment }\end{array}$ & $\begin{array}{c}\text { Water } \\
\text { level } \\
\text { altitude } \\
\text { (feet } \\
\text { above } \\
\text { NGVD 29) }\end{array}$ & Remarks \\
\hline FL & USGS-FLWSC & SR 316 WEST OF FAIRFIELD & 292146082182501 & - & Marion & 292140 & 821825 & $5 / 25 / 2010$ & 48 & \\
\hline FL & USGS-FLWSC & $\begin{array}{l}\text { SR52 6" UFA near Fivay Junc- } \\
\text { tion }\end{array}$ & 281949082332001 & - & Pasco & 281949 & 823319 & $5 / 18 / 2010$ & 53 & \\
\hline FL & USGS-FLWSC & $\begin{array}{l}\text { SR52 6" UFA near Gowers } \\
\text { Corner }\end{array}$ & 281918082264601 & - & Pasco & 281919 & 822645 & $5 / 18 / 2010$ & 70 & \\
\hline FL & USGS-FLWSC & SR52 6" UFA near Hudson & 282009082373801 & - & Pasco & 282010 & 823737 & $5 / 18 / 2010$ & 25 & \\
\hline FL & USGS-FLWSC & $\begin{array}{l}\text { SR54 6" UFA near Land O' } \\
\text { Lakes }\end{array}$ & 281143082304702 & - & Pasco & 281144 & 823046 & $5 / 18 / 2010$ & 51 & \\
\hline FL & USGS-FLWSC & $\begin{array}{l}\text { SR577 6" UFA near San } \\
\text { Antonio }\end{array}$ & 281715082164401 & - & Pasco & 281716 & 821643 & $6 / 4 / 2010$ & 86 & \\
\hline FL & USGS-FLWSC & $\begin{array}{l}\text { St. Joe, SR60 UFA near Lake } \\
\text { Wales }\end{array}$ & 275403081391301 & - & Polk & 275404 & 813912 & $5 / 20 / 2010$ & 99 & \\
\hline FL & USGS-FLWSC & $\begin{array}{l}\text { Starkey 8" MW-1 UFA near } \\
\text { Elfers }\end{array}$ & 281446082354101 & - & Pasco & 281447 & 823540 & $5 / 20 / 2010$ & 35 & \\
\hline FL & USGS-FLWSC & $\begin{array}{l}\text { Starkey NOP NPMW 10d near } \\
\text { New Port Richey, FL }\end{array}$ & 281729082330401 & - & Pasco & 281729 & 823304 & $5 / 19 / 2010$ & 51 & \\
\hline FL & USGS-FLWSC & $\begin{array}{l}\text { Starkey NOP NPMW 7d near } \\
\text { New Port Richey, FL }\end{array}$ & 281823082341101 & - & Pasco & 281823 & 823411 & $5 / 20 / 2010$ & 45 & \\
\hline FL & USGS-FLWSC & $\begin{array}{l}\text { Starkey NOP-NPMW-11d near } \\
\text { New Port Richey, FL }\end{array}$ & 281635082342101 & - & Pasco & 281635 & 823421 & $5 / 20 / 2010$ & 43 & \\
\hline FL & USGS-FLWSC & Starkey UFA \#10 near Elfers & 281451082380701 & - & Pasco & 281452 & 823806 & $5 / 20 / 2010$ & 29 & \\
\hline FL & USGS-FLWSC & $\begin{array}{l}\text { Stephens UFA \#72 10" UFA } \\
\text { near Ona }\end{array}$ & 272442082015201 & - & Hardee & 272443 & 820151 & $5 / 17 / 2010$ & 17 & \\
\hline FL & USGS-FLWSC & $\begin{array}{l}\text { STUART RANCH REPLACE- } \\
\text { MENT/REPLACEMENT }\end{array}$ & 284057081593601 & - & Sumter & 284058 & 815935 & $5 / 18 / 2010$ & 82 & \\
\hline FL & USGS-FLWSC & $\begin{array}{l}\text { SUMTER } 13 \text { REPLACEMENT } \\
\text { NR WILDWOOD }\end{array}$ & 285119082120601 & - & Sumter & 285120 & 821205 & $5 / 19 / 2010$ & 42 & \\
\hline
\end{tabular}


Table 1. Water-level measurements in Florida and parts of Georgia, South Carolina, and Alabama, May-June 2010.—Continued

[NGVD 29, National Geodetic Vertical Datum of 1929. Abbreviations for agency database: ACEPD, Alachua County Environmental Protection Department; FGS, Florida Geological Survey; GSA, Geological Survey of Alabama; NWFWMD, Northwest Florida Water Management District; SFWMD, South Florida Water Management District; SRWMD, Suwannee River Water Management District; USGSALWSC, U.S. Geological Survey Alabama Water Science Center; USGS-FLWSC, U.S. Geological Survey Florida Water Science Center; GAWSC, U.S. Geological Survey Georgia Water Science Center; SCWSC, U.S. Geological Survey South Carolina Water Science Center. Abbreviations for Station names: BLVD., boulevard; CO, county; CR, county road; E, east; HS, high school; IFAS, Institute of Food and Agricultural Services; LK, lake; MW, monitoring well; N, north; NE, northeast; NE, northeast; NF, national forest; NW, northwest; PK, park; R., river; RD., road; SE, southeast; SE, southeast; SR, state road; ST., street; TWR, tower; UFA, Upper Floridan aquifer; - , not available; \#, number; “, inches]

\begin{tabular}{|c|c|c|c|c|c|c|c|c|c|c|}
\hline State & $\begin{array}{l}\text { Agency } \\
\text { database }\end{array}$ & Station name & $\begin{array}{c}\text { USGS site } \\
\text { identification } \\
\text { number }\end{array}$ & $\begin{array}{l}\text { Local agency } \\
\text { unique site } \\
\text { identifier }\end{array}$ & County & $\begin{array}{l}\text { Latitude } \\
\text { (degree } \\
\text { minutes } \\
\text { and } \\
\text { seconds) } \\
\text { [DDMMSS] }\end{array}$ & $\begin{array}{l}\text { Longitude } \\
\text { (degree } \\
\text { minutes } \\
\text { and } \\
\text { seconds) } \\
\text { [DDMMSS] }\end{array}$ & $\begin{array}{c}\text { Date of } \\
\text { measure- } \\
\text { ment }\end{array}$ & $\begin{array}{c}\text { Water } \\
\text { level } \\
\text { altitude } \\
\text { (feet } \\
\text { above } \\
\text { NGVD 29) }\end{array}$ & Remarks \\
\hline FL & USGS-FLWSC & $\begin{array}{l}\text { SW Hills UFA \#71 UFA near } \\
\text { Ruskin }\end{array}$ & 274303082280901 & - & Hillsborough & 274304 & 822808 & $5 / 20 / 2010$ & 10 & \\
\hline FL & USGS-FLWSC & $\begin{array}{l}\text { SW Hillsborough UFA \#220, } \\
\text { Adamsville }\end{array}$ & 274928082225501 & - & Hillsborough & 274929 & 822254 & $5 / 19 / 2010$ & 6 & \\
\hline FL & USGS-FLWSC & $\begin{array}{l}\text { SWFWMD 6" UFA Test UFA } \\
\text { \#1, Dunedin }\end{array}$ & 280129082445501 & - & Pinellas & 280130 & 824454 & $5 / 18 / 2010$ & 6 & \\
\hline FL & USGS-FLWSC & T \& J RANCH 4" UFA, SR 336 & 290503082323101 & - & Levy & 290504 & 823230 & $5 / 26 / 2010$ & 71 & \\
\hline FL & USGS-FLWSC & $\begin{array}{l}\text { T-1 6" UFA near Crystal } \\
\text { Springs }\end{array}$ & 280708082074801 & - & Hillsborough & 280709 & 820747 & $5 / 13 / 2010$ & 88 & \\
\hline FL & USGS-FLWSC & $\begin{array}{l}\text { T-2 6" UFA near Crystal } \\
\text { Springs }\end{array}$ & 280510082043801 & - & Hillsborough & 280511 & 820437 & $5 / 12 / 2010$ & 98 & \\
\hline FL & USGS-FLWSC & $\begin{array}{l}\text { T-3 6" UFA near Crystal } \\
\text { Springs }\end{array}$ & 280849082053701 & - & Hillsborough & 280850 & 820536 & $5 / 13 / 2010$ & 93 & \\
\hline FL & USGS-FLWSC & $\begin{array}{l}\text { Tampa 16" UFA \#15 near } \\
\text { Dover }\end{array}$ & 280145082132501 & - & Hillsborough & 280146 & 821324 & $5 / 17 / 2010$ & 60 & \\
\hline FL & USGS-FLWSC & Tampa Stables 4" UFA, Tampa & 275316082285901 & - & Hillsborough & 275317 & 822858 & $5 / 20 / 2010$ & 2 & \\
\hline FL & USGS-FLWSC & $\begin{array}{l}\text { Tarpon Rd 6" UFA near Tarpon } \\
\text { Springs }\end{array}$ & 280907082424801 & - & Pinellas & 280908 & 824247 & $5 / 19 / 2010$ & 9 & \\
\hline FL & USGS-FLWSC & $\begin{array}{l}\text { Tbc-05 Tampa UFA Nr Temple } \\
\text { Terrace }\end{array}$ & 280012082204901 & - & Hillsborough & 280013 & 822048 & $5 / 19 / 2010$ & 18 & \\
\hline FL & USGS-FLWSC & $\begin{array}{l}\text { TENNOROCK ROAD WELL } \\
\text { NR LAKELAND }\end{array}$ & 280556081532601 & - & Polk & 280557 & 815325 & $5 / 19 / 2010$ & 118 & \\
\hline FL & USGS-FLWSC & $\begin{array}{l}\text { TH-3 OS0051 4" UFA, LAKE } \\
\text { POINSETT SW }\end{array}$ & 281630080591001 & - & Osceola & 281631 & 805909 & $5 / 18 / 2010$ & 37 & \\
\hline FL & USGS-FLWSC & $\begin{array}{l}\text { TH-9 OS0052 4" UFA, NOVA } \\
\text { RD } 532 \text { WEST }\end{array}$ & 281630081024401 & - & Osceola & 281631 & 810243 & $5 / 18 / 2010$ & 41 & \\
\hline
\end{tabular}


Table 1. Water-level measurements in Florida and parts of Georgia, South Carolina, and Alabama, May-June 2010.-Continued

[NGVD 29, National Geodetic Vertical Datum of 1929. Abbreviations for agency database: ACEPD, Alachua County Environmental Protection Department; FGS, Florida Geological Survey; GSA, Geological Survey of Alabama; NWFWMD, Northwest Florida Water Management District; SFWMD, South Florida Water Management District; SRWMD, Suwannee River Water Management District; USGSALWSC, U.S. Geological Survey Alabama Water Science Center; USGS-FLWSC, U.S. Geological Survey Florida Water Science Center; GAWSC, U.S. Geological Survey Georgia Water Science Center; SCWSC, U.S. Geological Survey South Carolina Water Science Center. Abbreviations for Station names: BLVD., boulevard; CO, county; CR, county road; E, east; HS, high school; IFAS, Institute of Food and Agricultural Services; LK, lake; MW, monitoring well; N, north; NE, northeast; NE, northeast; NF, national forest; NW, northwest; PK, park; R., river; RD., road; SE, southeast; SE, southeast; SR, state road; ST., street; TWR, tower; UFA, Upper Floridan aquifer; - - not available; \#, number; “, inches]

\begin{tabular}{|c|c|c|c|c|c|c|c|c|c|c|}
\hline State & $\begin{array}{c}\text { Agency } \\
\text { database }\end{array}$ & Station name & $\begin{array}{c}\text { USGS site } \\
\text { identification } \\
\text { number }\end{array}$ & $\begin{array}{l}\text { Local agency } \\
\text { unique site } \\
\text { identifier }\end{array}$ & County & $\begin{array}{l}\text { Latitude } \\
\text { (degree } \\
\text { minutes } \\
\text { and } \\
\text { seconds) } \\
\text { [DDMMS] }\end{array}$ & $\begin{array}{l}\text { Longitude } \\
\text { (degree } \\
\text { minutes } \\
\text { and } \\
\text { seconds) } \\
\text { [DDMMSS] }\end{array}$ & $\begin{array}{c}\text { Date of } \\
\text { measure- } \\
\text { ment }\end{array}$ & $\begin{array}{c}\text { Water } \\
\text { level } \\
\text { altitude } \\
\text { (feet } \\
\text { above } \\
\text { NGVD 29) }\end{array}$ & Remarks \\
\hline FL & USGS-FLWSC & $\begin{array}{l}\text { Tides Golf Course 8" UFA near } \\
\text { Seminole }\end{array}$ & 274937082480801 & - & Pinellas & 274938 & 824807 & $5 / 17 / 2010$ & 7 & \\
\hline FL & USGS-FLWSC & TIDEWATER 12" UFA\#1 & 290743082341501 & -151734001 & Levy & 290744 & 823413 & $5 / 26 / 2010$ & 54 & $\begin{array}{l}\text { SRWMD Site iden- } \\
\text { tification number } \\
-151734001\end{array}$ \\
\hline FL & USGS-FLWSC & TOMKOW HAY BARN WELL & 282816082123701 & - & Pasco & 282817 & 821236 & $5 / 17 / 2010$ & 51 & \\
\hline FL & USGS-FLWSC & TOWN OF WEBSTER UFA 2 & 283638082025702 & - & Sumter & 283639 & 820256 & $5 / 17 / 2010$ & 82 & \\
\hline FL & USGS-FLWSC & TR 18-2 Aripeka UFA & 282659082391104 & - & Hernando & 282660 & 823910 & $5 / 17 / 2010$ & 8 & \\
\hline FL & USGS-FLWSC & $\begin{array}{l}\text { TR12-3 Southernconfort } 6 " \\
\text { UFA }\end{array}$ & 280005082324201 & - & Hillsborough & 280006 & 823241 & $5 / 20 / 2010$ & 11 & \\
\hline FL & USGS-FLWSC & TR13-1X 4" UFA, E Bay & 275458082464002 & - & Pinellas & 275459 & 824639 & $5 / 17 / 2010$ & 6 & \\
\hline FL & USGS-FLWSC & $\begin{array}{l}\text { TRAILER PARK WELL ON } \\
\text { LAKE ROSALI }\end{array}$ & 275622081252301 & - & Polk & 275623 & 812522 & $5 / 18 / 2010$ & 59 & \\
\hline FL & USGS-FLWSC & TRAIL'S END WELL & 284439082131401 & - & Citrus & 284440 & 821313 & $5 / 19 / 2010$ & 40 & \\
\hline FL & USGS-FLWSC & $\begin{array}{l}\text { Travelers Rest 4" UFA near } \\
\text { Darby }\end{array}$ & 282434082200301 & - & Pasco & 282435 & 822002 & $5 / 17 / 2010$ & 61 & \\
\hline FL & USGS-FLWSC & Trg J36 12" UFA near Arcadia & 271748081345101 & - & De Soto & 271749 & 813450 & $5 / 20 / 2010$ & 46 & \\
\hline FL & USGS-FLWSC & Turner UFA near Brandon & 275627082150801 & - & Hillsborough & 275628 & 821507 & $5 / 17 / 2010$ & 18 & \\
\hline FL & USGS-FLWSC & $\begin{array}{l}\text { US } 98 \text { WELL NEAR DADE } \\
\text { CITY }\end{array}$ & 281654082065901 & - & Pasco & 281655 & 820658 & $5 / 17 / 2010$ & 77 & \\
\hline FL & USGS-FLWSC & $\begin{array}{l}\text { US Phosphoric 8" UFA, Riv- } \\
\text { erview }\end{array}$ & 275215082201901 & - & Hillsborough & 275216 & 822018 & $5 / 19 / 2010$ & 10 & \\
\hline FL & USGS-FLWSC & $\begin{array}{l}\text { USCE Test TBC-01 6" UFA } \\
\text { near Temple Terrace }\end{array}$ & 280243082203701 & - & Hillsborough & 280244 & 822036 & $5 / 19 / 2010$ & 23 & \\
\hline FL & USGS-FLWSC & $\begin{array}{l}\text { USGS (Morris Bridge) 1" UFA } \\
\quad \# 532 \text { near Branchton }\end{array}$ & 280926082162101 & - & Hillsborough & 280927 & 821620 & $5 / 17 / 2010$ & 44 & \\
\hline
\end{tabular}


Table 1. Water-level measurements in Florida and parts of Georgia, South Carolina, and Alabama, May-June 2010.—Continued

[NGVD 29, National Geodetic Vertical Datum of 1929. Abbreviations for agency database: ACEPD, Alachua County Environmental Protection Department; FGS, Florida Geological Survey; GSA, Geological Survey of Alabama; NWFWMD, Northwest Florida Water Management District; SFWMD, South Florida Water Management District; SRWMD, Suwannee River Water Management District; USGSALWSC, U.S. Geological Survey Alabama Water Science Center; USGS-FLWSC, U.S. Geological Survey Florida Water Science Center; GAWSC, U.S. Geological Survey Georgia Water Science Center; SCWSC, U.S. Geological Survey South Carolina Water Science Center. Abbreviations for Station names: BLVD., boulevard; CO, county; CR, county road; E, east; HS, high school; IFAS, Institute of Food and Agricultural Services; LK, lake; MW, monitoring well; N, north; NE, northeast; NE, northeast; NF, national forest; NW, northwest; PK, park; R., river; RD., road; SE, southeast; SE, southeast; SR, state road; ST., street; TWR, tower; UFA, Upper Floridan aquifer; —, not available; \#, number; “, inches]

\begin{tabular}{|c|c|c|c|c|c|c|c|c|c|c|}
\hline State & $\begin{array}{l}\text { Agency } \\
\text { database }\end{array}$ & Station name & $\begin{array}{c}\text { USGS site } \\
\text { identification } \\
\text { number }\end{array}$ & $\begin{array}{l}\text { Local agency } \\
\text { unique site } \\
\text { identifier }\end{array}$ & County & $\begin{array}{l}\text { Latitude } \\
\text { (degree } \\
\text { minutes } \\
\text { and } \\
\text { seconds) } \\
\text { [DDMMSS] }\end{array}$ & $\begin{array}{l}\text { Longitude } \\
\text { (degree } \\
\text { minutes } \\
\text { and } \\
\text { seconds) } \\
\text { ] [DDMMSS] }\end{array}$ & $\begin{array}{c}\text { Date of } \\
\text { measure- } \\
\text { ment }\end{array}$ & $\begin{array}{l}\text { Water } \\
\text { level } \\
\text { altitude } \\
\text { (feet } \\
\text { above } \\
\text { NGVD 29) }\end{array}$ & Remarks \\
\hline FL & USGS-FLWSC & $\begin{array}{l}\text { USGS 4" UFA near Myakka } \\
\text { Head }\end{array}$ & 272735082083401 & - & Manatee & 272736 & 820833 & $5 / 17 / 2010$ & 4 & \\
\hline FL & USGS-FLWSC & USGS 4" UFA near Osteen & 285221081095002 & - & Volusia & 285222 & 810949 & $5 / 25 / 2010$ & 23 & \\
\hline FL & USGS-FLWSC & USGS 6" UFA, ROSEWOOD & 291414082560901 & - & Levy & 291415 & 825608 & $5 / 26 / 2010$ & 11 & \\
\hline FL & USGS-FLWSC & $\begin{array}{l}\text { USGS CE } 89 \text { US 19-98,S OF } \\
\text { CFBC }\end{array}$ & 290023082393601 & - & Citrus & 290024 & 823935 & $5 / 20 / 2010$ & 14 & \\
\hline FL & USGS-FLWSC & $\begin{array}{l}\text { USGS OB WELL AT L WEO- } \\
\text { HYAKAPKA }\end{array}$ & 274846081262001 & - & Polk & 274847 & 812619 & $5 / 18 / 2010$ & 81 & \\
\hline FL & USGS-FLWSC & $\begin{array}{l}\text { USGS OB WELL GL155 NR } \\
\text { BRIGHTON }\end{array}$ & 271150081054401 & - & Glades & 271151 & 810543 & $5 / 18 / 2010$ & 46 & $\begin{array}{l}\text { Uncorrected hydrau- } \\
\text { lic head reported; } \\
\text { brackish to saline } \\
\text { water; not con- } \\
\text { toured on Potentio- } \\
\text { metric surface map }\end{array}$ \\
\hline FL & USGS-FLWSC & $\begin{array}{l}\text { USGS SH WELL W5470 NR } \\
\text { POLK CITY }\end{array}$ & 281532081493001 & - & Polk & 281533 & 814929 & $5 / 19 / 2010$ & 124 & \\
\hline FL & USGS-FLWSC & $\begin{array}{l}\text { USGS Tampa 2" UFA \#402 } \\
\text { near Tampa }\end{array}$ & 280420082285501 & - & Hillsborough & 280421 & 822854 & $5 / 24 / 2010$ & 39 & \\
\hline FL & USGS-FLWSC & USGS TUCKER T-6 & 275138080491801 & - & Brevard & 275139 & 804924 & $5 / 18 / 2010$ & 42 & \\
\hline FL & USGS-FLWSC & $\begin{array}{l}\text { USGS UFA CE14 NR DUN- } \\
\text { NELLON }\end{array}$ & 290312082250801 & - & Marion & 290313 & 822507 & $5 / 24 / 2010$ & 42 & \\
\hline FL & USGS-FLWSC & $\begin{array}{l}\text { USGS UFA CE23 NR DUN- } \\
\text { NELLON }\end{array}$ & 290106082191001 & - & Marion & 290107 & 821909 & $5 / 24 / 2010$ & 43 & \\
\hline FL & USGS-FLWSC & $\begin{array}{l}\text { USGS WELL CE } 7 \text { NR CRYS- } \\
\text { TAL RIVER }\end{array}$ & 285812082360901 & - & Citrus & 285813 & 823608 & $5 / 20 / 2010$ & 12 & \\
\hline FL & USGS-FLWSC & $\begin{array}{l}\text { USGS WELL NR LAKE } \\
\text { SAWYER }\end{array}$ & 282738081341401 & - & Orange & 282739 & 813413 & $5 / 18 / 2010$ & 81 & \\
\hline
\end{tabular}


Table 1. Water-level measurements in Florida and parts of Georgia, South Carolina, and Alabama, May-June 2010.-Continued

[NGVD 29, National Geodetic Vertical Datum of 1929. Abbreviations for agency database: ACEPD, Alachua County Environmental Protection Department; FGS, Florida Geological Survey; GSA, Geological Survey of Alabama; NWFWMD, Northwest Florida Water Management District; SFWMD, South Florida Water Management District; SRWMD, Suwannee River Water Management District; USGSALWSC, U.S. Geological Survey Alabama Water Science Center; USGS-FLWSC, U.S. Geological Survey Florida Water Science Center; GAWSC, U.S. Geological Survey Georgia Water Science Center; SCWSC, U.S. Geological Survey South Carolina Water Science Center. Abbreviations for Station names: BLVD., boulevard; CO, county; CR, county road; E, east; HS, high school; IFAS, Institute of Food and Agricultural Services; LK, lake; MW, monitoring well; N, north; NE, northeast; NE, northeast; NF, national forest; NW, northwest; PK, park; R., river; RD., road; SE, southeast; SE, southeast; SR, state road; ST., street; TWR, tower; UFA, Upper Floridan aquifer; - - not available; \#, number; “, inches]

\begin{tabular}{|c|c|c|c|c|c|c|c|c|c|c|}
\hline State & $\begin{array}{c}\text { Agency } \\
\text { database }\end{array}$ & Station name & $\begin{array}{c}\text { USGS site } \\
\text { identification } \\
\text { number }\end{array}$ & $\begin{array}{c}\text { Local agency } \\
\text { unique site } \\
\text { identifier }\end{array}$ & County & $\begin{array}{l}\text { Latitude } \\
\text { (degree } \\
\text { minutes } \\
\text { and } \\
\text { seconds) } \\
\text { [DDMMSS] }\end{array}$ & $\begin{array}{l}\text { Longitude } \\
\text { (degree } \\
\text { minutes } \\
\text { and } \\
\text { seconds) } \\
\text { [DDMMSS] }\end{array}$ & $\begin{array}{c}\text { Date of } \\
\text { measure- } \\
\text { ment }\end{array}$ & $\begin{array}{c}\text { Water } \\
\text { level } \\
\text { altitude } \\
\text { (feet } \\
\text { above } \\
\text { NGVD 29) }\end{array}$ & Remarks \\
\hline FL & USGS-FLWSC & $\begin{array}{l}\text { USGS WELL PALM LAKE } \\
\text { DR }\end{array}$ & 282835081305201 & - & Orange & 282839 & 813025 & $5 / 17 / 2010$ & 68 & \\
\hline FL & USGS-FLWSC & $\begin{array}{l}\text { V-0068 4" UFA, } 1 \text { mi. W of } \\
\text { Pierson }\end{array}$ & 291458081294201 & - & Volusia & 291459 & 812941 & $5 / 25 / 2010$ & 18 & \\
\hline FL & USGS-FLWSC & $\begin{array}{l}\text { V-0081 USGS } 04 \text { UFA NR } \\
\text { DELAND }\end{array}$ & 290541081132902 & - & Volusia & 290542 & 811328 & $5 / 27 / 2010$ & 36 & \\
\hline FL & USGS-FLWSC & V-0083 USGS WELL & 285638081203101 & - & Volusia & 285639 & 812030 & $5 / 27 / 2010$ & 8 & \\
\hline FL & USGS-FLWSC & $\begin{array}{l}\text { V-0086 TIGER BAY TEST } \\
\text { WELL }\end{array}$ & 291006081101004 & - & Volusia & 291007 & 811009 & $5 / 27 / 2010$ & 26 & \\
\hline FL & USGS-FLWSC & $\begin{array}{l}\text { V-0096 R NOLAN WELL NR } \\
\text { SEVILLE }\end{array}$ & 291905081251001 & - & Volusia & 291906 & 812509 & $5 / 26 / 2010$ & 18 & \\
\hline FL & USGS-FLWSC & $\begin{array}{l}\text { V-0101 USGS 6" UFA, Ala- } \\
\text { mana }\end{array}$ & 285745081054001 & - & Volusia & 285706 & 810539 & $5 / 25 / 2010$ & 28 & \\
\hline FL & USGS-FLWSC & $\begin{array}{l}\text { V-0110 USGS 3" UFA 10, S. } \\
\text { SAMSULA }\end{array}$ & 285934081041801 & - & Volusia & 285935 & 810417 & $5 / 25 / 2010$ & 24 & \\
\hline FL & USGS-FLWSC & $\begin{array}{l}\text { V-0115 USGS J-2 4" UFA, W. } \\
\text { DELAND }\end{array}$ & 290138081203202 & - & Volusia & 290139 & 812031 & $5 / 26 / 2010$ & 11 & \\
\hline FL & USGS-FLWSC & $\begin{array}{l}\text { V-0117 USGS 3" UFA \#9, N. } \\
\text { SAMSULA }\end{array}$ & 290225081040301 & - & Volusia & 290226 & 810402 & $5 / 25 / 2010$ & 20 & \\
\hline FL & USGS-FLWSC & V-0118 USGS 3" UFA 5 & 290230081123401 & - & Volusia & 290231 & 811233 & $5 / 27 / 2010$ & 36 & \\
\hline FL & USGS-FLWSC & $\begin{array}{l}\text { V-0155 USCE } 426 \text { Stoughton } \\
\text { 6" UFA Artesian, Pine Island }\end{array}$ & 291835081324201 & - & Volusia & 291836 & 813241 & $5 / 26 / 2010$ & 6 & \\
\hline FL & USGS-FLWSC & $\begin{array}{l}\text { V-0162 PT ORANGE 3" UFA } \\
\text { 2, S OF WELLFIELD }\end{array}$ & 290806081013901 & - & Volusia & 290807 & 810138 & $5 / 25 / 2010$ & 1 & \\
\hline FL & USGS-FLWSC & $\begin{array}{l}\text { V-0188 TOMOKA TOWER } \\
\text { OCALA }\end{array}$ & 290834081073802 & - & Volusia & 290835 & 810737 & $5 / 27 / 2010$ & 17 & \\
\hline FL & USGS-FLWSC & V-0196 ORANGE CITY UFA & 285442081181401 & - & Volusia & 285443 & 811813 & $5 / 27 / 2010$ & 21 & \\
\hline
\end{tabular}


Table 1. Water-level measurements in Florida and parts of Georgia, South Carolina, and Alabama, May-June 2010.—Continued

[NGVD 29, National Geodetic Vertical Datum of 1929. Abbreviations for agency database: ACEPD, Alachua County Environmental Protection Department; FGS, Florida Geological Survey; GSA, Geological Survey of Alabama; NWFWMD, Northwest Florida Water Management District; SFWMD, South Florida Water Management District; SRWMD, Suwannee River Water Management District; USGSALWSC, U.S. Geological Survey Alabama Water Science Center; USGS-FLWSC, U.S. Geological Survey Florida Water Science Center; GAWSC, U.S. Geological Survey Georgia Water Science Center; SCWSC, U.S. Geological Survey South Carolina Water Science Center. Abbreviations for Station names: BLVD., boulevard; CO, county; CR, county road; E, east; HS, high school; IFAS, Institute of Food and Agricultural Services; LK, lake; MW, monitoring well; N, north; NE, northeast; NE, northeast; NF, national forest; NW, northwest; PK, park; R., river; RD., road; SE, southeast; SE, southeast; SR, state road; ST., street; TWR, tower; UFA, Upper Floridan aquifer; —, not available; \#, number; “, inches]

\begin{tabular}{|c|c|c|c|c|c|c|c|c|c|c|}
\hline State & $\begin{array}{l}\text { Agency } \\
\text { database }\end{array}$ & Station name & $\begin{array}{c}\text { USGS site } \\
\text { identification } \\
\text { number }\end{array}$ & $\begin{array}{l}\text { Local agency } \\
\text { unique site } \\
\text { identifier }\end{array}$ & County & $\begin{array}{l}\text { Latitude } \\
\text { (degree } \\
\text { minutes } \\
\text { and } \\
\text { seconds) } \\
\text { [DDMMSS] }\end{array}$ & $\begin{array}{l}\text { Longitude } \\
\text { (degree } \\
\text { minutes } \\
\text { and } \\
\text { seconds) } \\
\text { [DDMMSS] }\end{array}$ & $\begin{array}{c}\text { Date of } \\
\text { measure- } \\
\text { ment }\end{array}$ & $\begin{array}{c}\text { Water } \\
\text { level } \\
\text { altitude } \\
\text { (feet } \\
\text { above } \\
\text { NGVD 29) }\end{array}$ & Remarks \\
\hline FL & USGS-FLWSC & $\begin{array}{l}\text { V-0198 LAKE ASHBY TWR } \\
\text { UFA }\end{array}$ & 285419081041001 & - & Volusia & 285420 & 810409 & $5 / 25 / 2010$ & 16 & \\
\hline FL & USGS-FLWSC & $\begin{array}{l}\text { V-0508 NEW SMYRNA } \\
\text { BEACH }\end{array}$ & 290103080551902 & - & Volusia & 290104 & 805518 & $5 / 25 / 2010$ & 4 & \\
\hline FL & USGS-FLWSC & V-0531 PIERSON AP UFA & 291448081274905 & - & Volusia & 291449 & 812748 & $5 / 26 / 2010$ & 24 & \\
\hline FL & USGS-FLWSC & $\begin{array}{l}\text { V-0567 PATTY WIGGINS BR } \\
\text { 6" UFA }\end{array}$ & 292038081315302 & - & Volusia & 292039 & 813152 & $5 / 26 / 2010$ & 30 & \\
\hline FL & USGS-FLWSC & $\begin{array}{l}\text { V-0700 ORMOND BEACH } \\
\text { DAN FORD }\end{array}$ & 291040081143701 & - & Volusia & 291041 & 811436 & $5 / 27 / 2010$ & 32 & \\
\hline FL & USGS-FLWSC & $\begin{array}{l}\text { V-0742 LEE AIRPORT UFA } \\
\text { SITE } 1312\end{array}$ & 290614081183301 & - & Volusia & 290615 & 811832 & $5 / 27 / 2010$ & 33 & \\
\hline FL & USGS-FLWSC & $\begin{array}{l}\text { V-0772 GALAXY MIDDLE } \\
\text { SCHOOL UFA }\end{array}$ & 285524081132403 & - & Volusia & 285544 & 811337 & $5 / 25 / 2010$ & 11 & \\
\hline FL & USGS-FLWSC & V-0777 LK HELEN UFA & 285813081142402 & - & Volusia & 285814 & 811423 & $5 / 25 / 2010$ & 18 & \\
\hline FL & USGS-FLWSC & $\begin{array}{l}\text { V-0808 WL LAWRENCE } \\
\text { 4IN UFA NR LAKE } \\
\text { DAUGHARTY }\end{array}$ & 290550081162601 & - & Volusia & 290551 & 811625 & $5 / 27 / 2010$ & 39 & \\
\hline FL & USGS-FLWSC & V-0818 OSTEEN RANCH & 284840081115701 & - & Volusia & 284841 & 811156 & $5 / 25 / 2010$ & 18 & \\
\hline FL & USGS-FLWSC & V-0840 MIGOR SHILOH RD & 284859080501002 & - & Volusia & 284860 & 805009 & $5 / 25 / 2010$ & 4 & \\
\hline FL & USGS-FLWSC & V-1030 DELEON SP 200 & 290828081215103 & - & Volusia & 290829 & 812150 & $5 / 26 / 2010$ & 18 & \\
\hline FL & USGS-FLWSC & $\begin{array}{l}\text { V-1091 6" UFA S OF BLUE } \\
\text { SPRINGS }\end{array}$ & 285513081202801 & - & Volusia & 285514 & 812027 & $5 / 27 / 2010$ & 20 & \\
\hline FL & USGS-FLWSC & $\begin{array}{l}\text { V-1094 NR ORMOND } \\
\text { BEACH }\end{array}$ & 291705081073502 & - & Volusia & 291706 & 810734 & $5 / 27 / 2010$ & 6 & \\
\hline FL & USGS-FLWSC & $\begin{array}{l}\text { V-4034 SJRWMD 6IN; 2MI W } \\
\text { PIERSON }\end{array}$ & 291509081302601 & - & Volusia & 291510 & 813025 & $5 / 25 / 2010$ & 14 & \\
\hline
\end{tabular}


Table 1. Water-level measurements in Florida and parts of Georgia, South Carolina, and Alabama, May-June 2010.-Continued

[NGVD 29, National Geodetic Vertical Datum of 1929. Abbreviations for agency database: ACEPD, Alachua County Environmental Protection Department; FGS, Florida Geological Survey; GSA, Geological Survey of Alabama; NWFWMD, Northwest Florida Water Management District; SFWMD, South Florida Water Management District; SRWMD, Suwannee River Water Management District; USGSALWSC, U.S. Geological Survey Alabama Water Science Center; USGS-FLWSC, U.S. Geological Survey Florida Water Science Center; GAWSC, U.S. Geological Survey Georgia Water Science Center; SCWSC, U.S. Geological Survey South Carolina Water Science Center. Abbreviations for Station names: BLVD., boulevard; CO, county; CR, county road; E, east; HS, high school; IFAS, Institute of Food and Agricultural Services; LK, lake; MW, monitoring well; N, north; NE, northeast; NE, northeast; NF, national forest; NW, northwest; PK, park; R., river; RD., road; SE, southeast; SE, southeast; SR, state road; ST., street; TWR, tower; UFA, Upper Floridan aquifer; - - not available; \#, number; “, inches]

\begin{tabular}{|c|c|c|c|c|c|c|c|c|c|c|}
\hline State & $\begin{array}{c}\text { Agency } \\
\text { database }\end{array}$ & Station name & $\begin{array}{c}\text { USGS site } \\
\text { identification } \\
\text { number }\end{array}$ & $\begin{array}{l}\text { Local agency } \\
\text { unique site } \\
\text { identifier }\end{array}$ & County & $\begin{array}{l}\text { Latitude } \\
\text { (degree } \\
\text { minutes } \\
\text { and } \\
\text { seconds) } \\
\text { [DDMMS] }\end{array}$ & $\begin{array}{l}\text { Longitude } \\
\text { (degree } \\
\text { minutes } \\
\text { and } \\
\text { seconds) } \\
\text { [DDMMSS] }\end{array}$ & $\begin{array}{l}\text { Date of } \\
\text { measure- } \\
\text { ment }\end{array}$ & $\begin{array}{c}\text { Water } \\
\text { level } \\
\text { altitude } \\
\text { (feet } \\
\text { above } \\
\text { NGVD 29) }\end{array}$ & Remarks \\
\hline FL & USGS-FLWSC & $\begin{array}{l}\text { Venice Gardens 2" UFA near } \\
\text { Venice }\end{array}$ & 270420082230502 & - & Sarasota & 270421 & 822304 & $5 / 21 / 2010$ & 22 & \\
\hline FL & USGS-FLWSC & $\begin{array}{l}\text { Verna Test UFA 0-1 6" UFA } \\
\text { near Verna }\end{array}$ & 272404082161701 & - & Manatee & 272405 & 821616 & $5 / 17 / 2010$ & 1 & \\
\hline FL & USGS-FLWSC & $\begin{array}{l}\text { VISA MONITORING WELL } \\
\text { M-0177 TOM'S PARK }\end{array}$ & 291239082082702 & - & Marion & 291241 & 820826 & $5 / 25 / 2010$ & 44 & \\
\hline FL & USGS-FLWSC & $\begin{array}{l}\text { VISA MONITORING WELL } \\
\text { M-0212 TUSCAWILLA } \\
\text { PARK }\end{array}$ & 291123082075402 & - & Marion & 291125 & 820753 & $5 / 25 / 2010$ & 45 & \\
\hline FL & USGS-FLWSC & $\begin{array}{l}\text { WCRWSA Grassy Gulch 8" } \\
\text { UFA near Lithia }\end{array}$ & 275158082085101 & - & Hillsborough & 275159 & 820850 & $5 / 18 / 2010$ & 26 & \\
\hline FL & USGS-FLWSC & $\begin{array}{l}\text { WCRWSA SC-1 8" UFA near } \\
\text { Lithia }\end{array}$ & 275152082121401 & - & Hillsborough & 275153 & 821213 & $5 / 18 / 2010$ & 16 & \\
\hline FL & USGS-FLWSC & $\begin{array}{l}\text { WCRWSA SCHM-1 8" UFA } \\
\text { near Lithia }\end{array}$ & 275034082134001 & - & Hillsborough & 275035 & 821339 & $5 / 20 / 2010$ & 16 & \\
\hline FL & USGS-FLWSC & $\begin{array}{l}\text { WCRWSA SCHM-11 8" UFA } \\
\text { near Lithia }\end{array}$ & 275323082080601 & - & Hillsborough & 275324 & 820805 & $5 / 18 / 2010$ & 34 & \\
\hline FL & USGS-FLWSC & $\begin{array}{l}\text { WCRWSA SCHM-2 8" UFA } \\
\text { near Lithia }\end{array}$ & 275613082094401 & - & Hillsborough & 275614 & 820943 & $5 / 20 / 2010$ & 49 & \\
\hline FL & USGS-FLWSC & $\begin{array}{l}\text { WCRWSA SCHM-3 8" UFA } \\
\text { near Lithia }\end{array}$ & 275547082044801 & - & Hillsborough & 275548 & 820447 & $5 / 20 / 2010$ & 59 & \\
\hline FL & USGS-FLWSC & $\begin{array}{l}\text { WCRWSA SCHM-4 8" UFA } \\
\text { near Lithia }\end{array}$ & 275146082084301 & - & Hillsborough & 275147 & 820842 & $5 / 18 / 2010$ & 25 & \\
\hline FL & USGS-FLWSC & $\begin{array}{l}\text { WCRWSA SCHM-4 8" UFA } \\
\text { near Lithia }\end{array}$ & 275235082033601 & - & Hillsborough & 275236 & 820335 & $5 / 20 / 2010$ & 52 & \\
\hline FL & USGS-FLWSC & $\begin{array}{l}\text { WCRWSA SCHM-5 8" UFA } \\
\text { near Lithia }\end{array}$ & 275100082042001 & - & Hillsborough & 275101 & 820419 & $5 / 20 / 2010$ & 48 & \\
\hline
\end{tabular}


Table 1. Water-level measurements in Florida and parts of Georgia, South Carolina, and Alabama, May-June 2010.—Continued

[NGVD 29, National Geodetic Vertical Datum of 1929. Abbreviations for agency database: ACEPD, Alachua County Environmental Protection Department; FGS, Florida Geological Survey; GSA, Geological Survey of Alabama; NWFWMD, Northwest Florida Water Management District; SFWMD, South Florida Water Management District; SRWMD, Suwannee River Water Management District; USGSALWSC, U.S. Geological Survey Alabama Water Science Center; USGS-FLWSC, U.S. Geological Survey Florida Water Science Center; GAWSC, U.S. Geological Survey Georgia Water Science Center; SCWSC, U.S. Geological Survey South Carolina Water Science Center. Abbreviations for Station names: BLVD., boulevard; CO, county; CR, county road; E, east; HS, high school; IFAS, Institute of Food and Agricultural Services; LK, lake; MW, monitoring well; N, north; NE, northeast; NE, northeast; NF, national forest; NW, northwest; PK, park; R., river; RD., road; SE, southeast; SE, southeast; SR, state road; ST., street; TWR, tower; UFA, Upper Floridan aquifer; —, not available; \#, number; “, inches]

\begin{tabular}{|c|c|c|c|c|c|c|c|c|c|c|}
\hline State & $\begin{array}{l}\text { Agency } \\
\text { database }\end{array}$ & Station name & $\begin{array}{c}\text { USGS site } \\
\text { identification } \\
\text { number }\end{array}$ & $\begin{array}{l}\text { Local agency } \\
\text { unique site } \\
\text { identifier }\end{array}$ & County & $\begin{array}{l}\text { Latitude } \\
\text { (degree } \\
\text { minutes } \\
\text { and } \\
\text { seconds) } \\
\text { [DDMMSS] }\end{array}$ & $\begin{array}{l}\text { Longitude } \\
\text { (degree } \\
\text { minutes } \\
\text { and } \\
\text { seconds) } \\
\text { [DDMMSS] }\end{array}$ & $\begin{array}{l}\text { Date of } \\
\text { measure- } \\
\text { ment }\end{array}$ & $\begin{array}{c}\text { Water } \\
\text { level } \\
\text { altitude } \\
\text { (feet } \\
\text { above } \\
\text { NGVD 29) }\end{array}$ & Remarks \\
\hline FL & USGS-FLWSC & $\begin{array}{l}\text { WCRWSA SCHM-6 UFA near } \\
\text { Lithia }\end{array}$ & 274925082084301 & - & Hillsborough & 274926 & 820842 & $5 / 20 / 2010$ & 29 & \\
\hline FL & USGS-FLWSC & $\begin{array}{l}\text { WCRWSA SCHM-7 8" UFA } \\
\text { near Lithia }\end{array}$ & 274941082115701 & - & Hillsborough & 274942 & 821156 & $5 / 20 / 2010$ & 18 & \\
\hline FL & USGS-FLWSC & $\begin{array}{l}\text { WCRWSA SCHM-8 8" UFA } \\
\text { near Lithia }\end{array}$ & 275336082125401 & - & Hillsborough & 275337 & 821253 & $5 / 19 / 2010$ & 13 & \\
\hline FL & USGS-FLWSC & $\begin{array}{l}\text { Weeki Wachee 4" UFA \#11 near } \\
\text { Masaryktown }\end{array}$ & 282636082221401 & - & Hernando & 282637 & 822213 & $5 / 18 / 2010$ & 36 & \\
\hline FL & USGS-FLWSC & $\begin{array}{l}\text { Weeki Wachee 4" UFA near } \\
\text { Weeki Wachee }\end{array}$ & 283201082315601 & - & Hernando & 283202 & 823155 & $5 / 17 / 2010$ & 14 & \\
\hline FL & USGS-FLWSC & $\begin{array}{l}\text { Weeki Wachee Springs near } \\
\text { Brooksville }\end{array}$ & 02310500 & - & Hernando & 283103 & 823424 & $5 / 17 / 2010$ & 8 & \\
\hline FL & USGS-FLWSC & $\begin{array}{l}\text { WEEMS 6" UFA NR BRON- } \\
\text { SON }\end{array}$ & 292310082373701 & - & Levy & 292311 & 823736 & $5 / 26 / 2010$ & 52 & \\
\hline FL & USGS-FLWSC & $\begin{array}{l}\text { WILLISTON AIRPORT 6" } \\
\text { UFA }\end{array}$ & 292143082282201 & - & Levy & 292144 & 822821 & $5 / 26 / 2010$ & 44 & \\
\hline FL & USGS-FLWSC & $\begin{array}{l}\text { Wilson Irrig 12" UFA near } \\
\text { Arcadia }\end{array}$ & 271405081453201 & - & De Soto & 271406 & 814531 & $5 / 20 / 2010$ & 46 & \\
\hline FL & USGS-FLWSC & $\begin{array}{l}\text { Winter Quarters RV Park 4" } \\
\text { UFA near Land O' Lakes }\end{array}$ & 281124082274101 & - & Pasco & 281125 & 822740 & $5 / 18 / 2010$ & 61 & \\
\hline FL & USGS-FLWSC & Wire Rd UFA & 281556082104701 & - & Pasco & 281557 & 821046 & $5 / 17 / 2010$ & 66 & \\
\hline FL & USGS-FLWSC & $\begin{array}{l}\text { WITH. ST FOR GR SWP } \\
\text { WELL NR BAY LK }\end{array}$ & 282741081585701 & - & Sumter & 282742 & 815856 & $5 / 17 / 2010$ & 94 & \\
\hline FL & USGS-FLWSC & $\begin{array}{l}\text { Wrw-D 6" UFA near Masaryk- } \\
\text { town }\end{array}$ & 282324082285101 & - & Pasco & 282325 & 822850 & $5 / 18 / 2010$ & 42 & \\
\hline FL & USGS-FLWSC & $\begin{array}{l}\text { WSF-HOLDER MINE CAMP- } \\
\text { GROUND }\end{array}$ & 284805082225701 & - & Citrus & 284806 & 822256 & $5 / 21 / 2010$ & 12 & \\
\hline
\end{tabular}


Table 1. Water-level measurements in Florida and parts of Georgia, South Carolina, and Alabama, May-June 2010.-Continued

[NGVD 29, National Geodetic Vertical Datum of 1929. Abbreviations for agency database: ACEPD, Alachua County Environmental Protection Department; FGS, Florida Geological Survey; GSA, Geological Survey of Alabama; NWFWMD, Northwest Florida Water Management District; SFWMD, South Florida Water Management District; SRWMD, Suwannee River Water Management District; USGSALWSC, U.S. Geological Survey Alabama Water Science Center; USGS-FLWSC, U.S. Geological Survey Florida Water Science Center; GAWSC, U.S. Geological Survey Georgia Water Science Center; SCWSC, U.S. Geological Survey South Carolina Water Science Center. Abbreviations for Station names: BLVD., boulevard; CO, county; CR, county road; E, east; HS, high school; IFAS, Institute of Food and Agricultural Services; LK, lake; MW, monitoring well; N, north; NE, northeast; NE, northeast; NF, national forest; NW, northwest; PK, park; R., river; RD., road; SE, southeast; SE, southeast; SR, state road; ST., street; TWR, tower; UFA, Upper Floridan aquifer; - - not available; \#, number; “, inches]

\begin{tabular}{|c|c|c|c|c|c|c|c|c|c|c|}
\hline State & $\begin{array}{c}\text { Agency } \\
\text { database }\end{array}$ & Station name & $\begin{array}{c}\text { USGS site } \\
\text { identification } \\
\text { number }\end{array}$ & $\begin{array}{l}\text { Local agency } \\
\text { unique site } \\
\text { identifier }\end{array}$ & County & $\begin{array}{l}\text { Latitude } \\
\text { (degree } \\
\text { minutes } \\
\text { and } \\
\text { seconds) } \\
\text { [DDMMSS] }\end{array}$ & $\begin{array}{l}\text { Longitude } \\
\text { (degree } \\
\text { minutes } \\
\text { and } \\
\text { seconds) } \\
\text { ] [DDMMSS] }\end{array}$ & $\begin{array}{c}\text { Date of } \\
\text { measure- } \\
\text { ment }\end{array}$ & $\begin{array}{c}\text { Water } \\
\text { level } \\
\text { altitude } \\
\text { (feet } \\
\text { above } \\
\text { NGVD 29) }\end{array}$ & Remarks \\
\hline FL & USGS-FLWSC & $\begin{array}{l}\text { WSF-PERRYMAN TRACT } \\
\text { WELL }\end{array}$ & 284844082282801 & - & Citrus & 284845 & 822827 & $5 / 20 / 2010$ & 7 & \\
\hline FL & USGS-FLWSC & WYNNHAVEN CAMP WELL & 284317082142601 & - & Sumter & 284318 & 821425 & $5 / 18 / 2010$ & 40 & \\
\hline FL & USGS-FLWSC & Zephyrhills Park UFA & 281353082110401 & - & Pasco & 281354 & 821103 & $5 / 17 / 2010$ & 66 & \\
\hline FL & USGS-FLWSC & Zephyrhills Prison UFA & 281138082120201 & - & Pasco & 281139 & 821201 & $5 / 17 / 2010$ & 61 & \\
\hline GA & USGS-GAWSC & $05 \mathrm{H} 021$ & 311212085003401 & - & Early & 311212 & 850034 & $5 / 20 / 2010$ & 169 & \\
\hline GA & USGS-GAWSC & 05J007 & 311526085000801 & - & Early & 311527 & 850008 & $5 / 20 / 2010$ & 180 & \\
\hline GA & USGS-GAWSC & $06 \mathrm{E} 023$ & 304758084551301 & - & Seminole & 304759 & 845513 & $5 / 22 / 2010$ & 73 & \\
\hline GA & USGS-GAWSC & 06F001 & 305356084534601 & - & Seminole & 305350 & 845355 & $5 / 23 / 2010$ & 86 & \\
\hline GA & USGS-GAWSC & 06F084 & 305657084554801 & - & Seminole & 305658 & 845548 & $5 / 22 / 2010$ & 105 & \\
\hline GA & USGS-GAWSC & 06G006 & 310427084591101 & - & Seminole & 310428 & 845911 & $5 / 23 / 2010$ & 112 & \\
\hline GA & USGS-GAWSC & 06H009 & 311108084564301 & - & Early & 311109 & 845643 & $5 / 21 / 2010$ & 181 & \\
\hline GA & USGS-GAWSC & $06 \mathrm{H} 013$ & 310800084563601 & - & Early & 310801 & 845636 & $5 / 21 / 2010$ & 156 & \\
\hline GA & USGS-GAWSC & 06H022 & 311206084532201 & - & Miller & 311206 & 845322 & $6 / 3 / 2010$ & 171 & \\
\hline GA & USGS-GAWSC & 06J009 & 311733084540101 & - & Early & 311540 & 845329 & $5 / 22 / 2010$ & 166 & \\
\hline GA & USGS-GAWSC & 06J010 & 311608084555101 & - & Early & 311608 & 845551 & $5 / 21 / 2010$ & 213 & \\
\hline GA & USGS-GAWSC & 07D004 & 304351084475701 & - & Decatur & 304352 & 844757 & $5 / 18 / 2010$ & 67 & \\
\hline GA & USGS-GAWSC & 07F003 & 305816084454201 & - & Seminole & 305818 & 844543 & $5 / 21 / 2010$ & 79 & \\
\hline GA & USGS-GAWSC & 07F004 & 305938084465301 & - & Seminole & 305939 & 844653 & $5 / 21 / 2010$ & 88 & \\
\hline GA & USGS-GAWSC & $07 \mathrm{G} 026$ & 310249084504501 & - & Seminole & 310249 & 845045 & $5 / 22 / 2010$ & 117 & \\
\hline GA & USGS-GAWSC & $07 \mathrm{G} 027$ & 310514084512101 & - & Miller & 310514 & 845121 & $5 / 21 / 2010$ & 160 & \\
\hline GA & USGS-GAWSC & 07G028 & 310017084503901 & - & Seminole & 310017 & 845039 & $5 / 22 / 2010$ & 106 & \\
\hline GA & USGS-GAWSC & 07H002 & 311009084495502 & - & Miller & 311009 & 844954 & $5 / 23 / 2010$ & 158 & \\
\hline GA & USGS-GAWSC & 07H012 & 311113084454701 & - & Miller & 311114 & 844546 & $6 / 3 / 2010$ & 148 & \\
\hline GA & USGS-GAWSC & 07H025 & 311459084502501 & - & Miller & 311459 & 845024 & $5 / 21 / 2010$ & 171 & \\
\hline
\end{tabular}


Table 1. Water-level measurements in Florida and parts of Georgia, South Carolina, and Alabama, May-June 2010.—Continued

[NGVD 29, National Geodetic Vertical Datum of 1929. Abbreviations for agency database: ACEPD, Alachua County Environmental Protection Department; FGS, Florida Geological Survey; GSA, Geological Survey of Alabama; NWFWMD, Northwest Florida Water Management District; SFWMD, South Florida Water Management District; SRWMD, Suwannee River Water Management District; USGSALWSC, U.S. Geological Survey Alabama Water Science Center; USGS-FLWSC, U.S. Geological Survey Florida Water Science Center; GAWSC, U.S. Geological Survey Georgia Water Science Center; SCWSC, U.S. Geological Survey South Carolina Water Science Center. Abbreviations for Station names: BLVD., boulevard; CO, county; CR, county road; E, east; HS, high school; IFAS, Institute of Food and Agricultural Services; LK, lake; MW, monitoring well; N, north; NE, northeast; NE, northeast; NF, national forest; NW, northwest; PK, park; R., river; RD., road; SE, southeast; SE, southeast; SR, state road; ST., street; TWR, tower; UFA, Upper Floridan aquifer; - , not available; \#, number; “, inches]

\begin{tabular}{|c|c|c|c|c|c|c|c|c|c|c|}
\hline State & $\begin{array}{l}\text { Agency } \\
\text { database }\end{array}$ & Station name & $\begin{array}{c}\text { USGS site } \\
\text { identification } \\
\text { number }\end{array}$ & $\begin{array}{l}\text { Local agency } \\
\text { unique site } \\
\text { identifier }\end{array}$ & County & $\begin{array}{l}\text { Latitude } \\
\text { (degree } \\
\text { minutes } \\
\text { and } \\
\text { seconds) } \\
\text { [DDMMSS] }\end{array}$ & $\begin{array}{l}\text { Longitude } \\
\text { (degree } \\
\text { minutes } \\
\text { and } \\
\text { seconds) } \\
\text { [DDMMSS] }\end{array}$ & $\begin{array}{c}\text { Date of } \\
\text { measure- } \\
\text { ment }\end{array}$ & $\begin{array}{c}\text { Water } \\
\text { level } \\
\text { altitude } \\
\text { (feet } \\
\text { above } \\
\text { NGVD 29) }\end{array}$ & Remarks \\
\hline GA & USGS-GAWSC & 07H026 & 311430084460701 & - & Miller & 311430 & 844606 & $5 / 21 / 2010$ & 136 & \\
\hline GA & USGS-GAWSC & 07H027 & 310752084503301 & - & Miller & 310752 & 845033 & $5 / 21 / 2010$ & 146 & \\
\hline GA & USGS-GAWSC & 07J012 & 311929084464301 & - & Early & 311930 & 844642 & $5 / 21 / 2010$ & 168 & \\
\hline GA & USGS-GAWSC & $08 \mathrm{D} 006$ & 304247084402001 & - & Decatur & 304248 & 844020 & $5 / 18 / 2010$ & 82 & \\
\hline GA & USGS-GAWSC & 08E038 & 304712084395801 & - & Decatur & 304712 & 843958 & $5 / 23 / 2010$ & 77 & \\
\hline GA & USGS-GAWSC & 08E039 & 304806084404101 & - & Decatur & 304807 & 844041 & $5 / 23 / 2010$ & 78 & \\
\hline GA & USGS-GAWSC & 08F006 & 305848084434801 & - & Decatur & 305849 & 844348 & $5 / 18 / 2010$ & 86 & \\
\hline GA & USGS-GAWSC & 08F018 & 305236084440701 & - & Decatur & 305237 & 844407 & $5 / 18 / 2010$ & 81 & \\
\hline GA & USGS-GAWSC & 08F512 & 305501084411001 & - & Decatur & 305501 & 844110 & $5 / 25 / 2010$ & 81 & \\
\hline GA & USGS-GAWSC & 08F513 & 305706084404401 & - & Decatur & 305706 & 844043 & $5 / 25 / 2010$ & 89 & \\
\hline GA & USGS-GAWSC & 08G001 & 310651084404501 & - & Miller & 310652 & 844044 & $5 / 23 / 2010$ & 131 & \\
\hline GA & USGS-GAWSC & 08G005 & 310136084411701 & - & Decatur & 310137 & 844117 & $5 / 18 / 2010$ & 100 & \\
\hline GA & USGS-GAWSC & 08G013 & 310251084384001 & - & Decatur & 310251 & 843840 & $5 / 25 / 2010$ & 106 & \\
\hline GA & USGS-GAWSC & 08H003 & 311241084442501 & - & Miller & 311242 & 844425 & $6 / 3 / 2010$ & 129 & \\
\hline GA & USGS-GAWSC & 08H009 & 311411084403401 & - & Miller & 311412 & 844033 & $5 / 22 / 2010$ & 174 & \\
\hline GA & USGS-GAWSC & 08H010 & 310952084404801 & - & Miller & 310953 & 844048 & $5 / 21 / 2010$ & 133 & \\
\hline GA & USGS-GAWSC & $08 \mathrm{~K} 001$ & 312232084391701 & - & Early & 312239 & 843917 & $5 / 23 / 2010$ & 225 & \\
\hline GA & USGS-GAWSC & $08 \mathrm{~K} 023$ & 312610084421901 & - & Calhoun & 312610 & 844219 & $5 / 20 / 2010$ & 231 & \\
\hline GA & USGS-GAWSC & 09D002 & 304149084315801 & - & Decatur & 304149 & 843158 & $5 / 25 / 2010$ & 60 & \\
\hline GA & USGS-GAWSC & 09E003 & 305223084351701 & - & Decatur & 305223 & 843517 & $5 / 18 / 2010$ & 85 & \\
\hline GA & USGS-GAWSC & 09E004 & 305223084351301 & - & Decatur & 305224 & 843513 & $5 / 18 / 2010$ & 85 & \\
\hline GA & USGS-GAWSC & 09E009 & 304750084332201 & - & Decatur & 304751 & 843321 & $5 / 18 / 2010$ & 85 & \\
\hline GA & USGS-GAWSC & 09F004 & 305752084302201 & - & Decatur & 305754 & 843022 & $5 / 18 / 2010$ & 83 & \\
\hline GA & USGS-GAWSC & 09F520 & 305736084355801 & - & Decatur & 305743 & 843546 & $5 / 23 / 2010$ & 79 & \\
\hline
\end{tabular}


Table 1. Water-level measurements in Florida and parts of Georgia, South Carolina, and Alabama, May-June 2010.—Continued

[NGVD 29, National Geodetic Vertical Datum of 1929. Abbreviations for agency database: ACEPD, Alachua County Environmental Protection Department; FGS, Florida Geological Survey; GSA, Geological Survey of Alabama; NWFWMD, Northwest Florida Water Management District; SFWMD, South Florida Water Management District; SRWMD, Suwannee River Water Management District; USGSALWSC, U.S. Geological Survey Alabama Water Science Center; USGS-FLWSC, U.S. Geological Survey Florida Water Science Center; GAWSC, U.S. Geological Survey Georgia Water Science Center; SCWSC, U.S. Geological Survey South Carolina Water Science Center. Abbreviations for Station names: BLVD., boulevard; CO, county; CR, county road; E, east; HS, high school; IFAS, Institute of Food and Agricultural Services; LK, lake; MW, monitoring well; N, north; NE, northeast; NE, northeast; NF, national forest; NW, northwest; PK, park; R., river; RD., road; SE, southeast; SE, southeast; SR, state road; ST., street; TWR, tower; UFA, Upper Floridan aquifer; - - not available; \#, number; “, inches]

\begin{tabular}{|c|c|c|c|c|c|c|c|c|c|c|}
\hline State & $\begin{array}{c}\text { Agency } \\
\text { database }\end{array}$ & Station name & $\begin{array}{c}\text { USGS site } \\
\text { identification } \\
\text { number }\end{array}$ & $\begin{array}{l}\text { Local agency } \\
\text { unique site } \\
\text { identifier }\end{array}$ & County & $\begin{array}{l}\text { Latitude } \\
\text { (degree } \\
\text { minutes } \\
\text { and } \\
\text { seconds) } \\
\text { [DDMMS] }\end{array}$ & $\begin{array}{l}\text { Longitude } \\
\text { (degree } \\
\text { minutes } \\
\text { and } \\
\text { seconds) } \\
\text { ] [DDMMSS] }\end{array}$ & $\begin{array}{c}\text { Date of } \\
\text { measure- } \\
\text { ment }\end{array}$ & $\begin{array}{c}\text { Water } \\
\text { level } \\
\text { altitude } \\
\text { (feet } \\
\text { above } \\
\text { NGVD 29) }\end{array}$ & Remarks \\
\hline GA & USGS-GAWSC & 09G001 & 310428084310501 & - & Decatur & 310429 & 843105 & $5 / 20 / 2010$ & 97 & \\
\hline GA & USGS-GAWSC & 09G005 & 310512084353201 & - & Miller & 310513 & 843531 & $6 / 3 / 2010$ & 117 & \\
\hline GA & USGS-GAWSC & 09G010 & 310250084342001 & - & Decatur & 310251 & 843421 & $5 / 18 / 2010$ & 94 & \\
\hline GA & USGS-GAWSC & 09H009 & 311416084335701 & - & Miller & 311417 & 843357 & $5 / 21 / 2010$ & 150 & \\
\hline GA & USGS-GAWSC & 09H012 & 310857084332701 & - & Miller & 310858 & 843327 & $6 / 3 / 2010$ & 111 & \\
\hline GA & USGS-GAWSC & 09J004 & 311823084341801 & - & Baker & 311823 & 843418 & $5 / 20 / 2010$ & 148 & \\
\hline GA & USGS-GAWSC & 09K010 & 312251084371701 & - & Baker & 312252 & 843717 & $5 / 20 / 2010$ & 192 & \\
\hline GA & USGS-GAWSC & 09K012 & 312919084313701 & - & Calhoun & 312919 & 843137 & $5 / 19 / 2010$ & 186 & \\
\hline GA & USGS-GAWSC & 09L029 & 313246084303201 & - & Calhoun & 313246 & 843032 & $5 / 19 / 2010$ & 227 & \\
\hline GA & USGS-GAWSC & 10D015 & 304457084290101 & - & Decatur & 304458 & 842901 & $5 / 18 / 2010$ & 75 & \\
\hline GA & USGS-GAWSC & 10F004 & 305822084263601 & - & Decatur & 305823 & 842636 & $5 / 18 / 2010$ & 97 & \\
\hline GA & USGS-GAWSC & 10G001 & 310117084231501 & - & Decatur & 310123 & 842314 & $5 / 18 / 2010$ & 93 & \\
\hline GA & USGS-GAWSC & 10G313 & 310507084262201 & - & Mitchell & 310508 & 842622 & $5 / 23 / 2010$ & 99 & \\
\hline GA & USGS-GAWSC & 10G317 & 310543084234901 & - & Mitchell & 310543 & 842349 & $5 / 25 / 2010$ & 105 & \\
\hline GA & USGS-GAWSC & $10 \mathrm{H} 004$ & 311243084292601 & - & Baker & 311243 & 842925 & $6 / 3 / 2010$ & 119 & \\
\hline GA & USGS-GAWSC & $10 \mathrm{H} 006$ & 310804084254401 & - & Mitchell & 310805 & 842544 & $6 / 3 / 2010$ & 100 & \\
\hline GA & USGS-GAWSC & $10 \mathrm{H} 009$ & 311400084295502 & - & Baker & 311401 & 842955 & $5 / 23 / 2010$ & 137 & \\
\hline GA & USGS-GAWSC & $10 \mathrm{H} 012$ & 310734084290101 & - & Mitchell & 310734 & 842901 & $6 / 3 / 2010$ & 91 & \\
\hline GA & USGS-GAWSC & 10J003 & 311806084233701 & - & Baker & 311806 & 842336 & $6 / 3 / 2010$ & 138 & \\
\hline GA & USGS-GAWSC & 10J010 & 312040084295301 & - & Baker & 312040 & 842953 & $5 / 25 / 2010$ & 139 & \\
\hline GA & USGS-GAWSC & $10 \mathrm{~K} 005$ & 312853084275101 & - & Calhoun & 312854 & 842751 & $5 / 23 / 2010$ & 167 & \\
\hline GA & USGS-GAWSC & 10L004 & 313532084283501 & - & Calhoun & 313533 & 842835 & $5 / 19 / 2010$ & 224 & \\
\hline GA & USGS-GAWSC & 10M019 & 314238084295301 & - & Terrell & 314238 & 842953 & $5 / 19 / 2010$ & 309 & \\
\hline GA & USGS-GAWSC & 10N024 & 314531084253501 & - & Terrell & 314532 & 842534 & $5 / 18 / 2010$ & 299 & \\
\hline
\end{tabular}


Table 1. Water-level measurements in Florida and parts of Georgia, South Carolina, and Alabama, May-June 2010.—Continued

[NGVD 29, National Geodetic Vertical Datum of 1929. Abbreviations for agency database: ACEPD, Alachua County Environmental Protection Department; FGS, Florida Geological Survey; GSA, Geological Survey of Alabama; NWFWMD, Northwest Florida Water Management District; SFWMD, South Florida Water Management District; SRWMD, Suwannee River Water Management District; USGSALWSC, U.S. Geological Survey Alabama Water Science Center; USGS-FLWSC, U.S. Geological Survey Florida Water Science Center; GAWSC, U.S. Geological Survey Georgia Water Science Center; SCWSC, U.S. Geological Survey South Carolina Water Science Center. Abbreviations for Station names: BLVD., boulevard; CO, county; CR, county road; E, east; HS, high school; IFAS, Institute of Food and Agricultural Services; LK, lake; MW, monitoring well; N, north; NE, northeast; NE, northeast; NF, national forest; NW, northwest; PK, park; R., river; RD., road; SE, southeast; SE, southeast; SR, state road; ST., street; TWR, tower; UFA, Upper Floridan aquifer; —, not available; \#, number; “, inches]

\begin{tabular}{|c|c|c|c|c|c|c|c|c|c|c|}
\hline State & $\begin{array}{c}\text { Agency } \\
\text { database }\end{array}$ & Station name & $\begin{array}{c}\text { USGS site } \\
\text { identification } \\
\text { number }\end{array}$ & $\begin{array}{l}\text { Local agency } \\
\text { unique site } \\
\text { identifier }\end{array}$ & County & $\begin{array}{l}\text { Latitude } \\
\text { (degree } \\
\text { minutes } \\
\text { and } \\
\text { seconds) } \\
\text { [DDMMSS] }\end{array}$ & $\begin{array}{l}\text { Longitude } \\
\text { (degree } \\
\text { minutes } \\
\text { and } \\
\text { seconds) } \\
\text { [DDMMSS] }\end{array}$ & $\begin{array}{c}\text { Date of } \\
\text { measure- } \\
\text { ment }\end{array}$ & $\begin{array}{c}\text { Water } \\
\text { level } \\
\text { altitude } \\
\text { (feet } \\
\text { above } \\
\text { NGVD 29) }\end{array}$ & Remarks \\
\hline GA & USGS-GAWSC & 11D027 & 304159084205501 & - & Grady & 304205 & 842056 & $5 / 17 / 2010$ & 62 & \\
\hline GA & USGS-GAWSC & 11F091 & 305331084163301 & - & Grady & 305331 & 841633 & $5 / 17 / 2010$ & 71 & \\
\hline GA & USGS-GAWSC & $11 \mathrm{G} 021$ & 310226084182801 & - & Grady & 310227 & 841828 & $5 / 17 / 2010$ & 110 & \\
\hline GA & USGS-GAWSC & $11 \mathrm{H} 003$ & 310830084215501 & - & Mitchell & 310831 & 842155 & $6 / 3 / 2010$ & 113 & \\
\hline GA & USGS-GAWSC & $11 \mathrm{~J} 003$ & 312129084201701 & - & Baker & 312131 & 842016 & $5 / 20 / 2010$ & 149 & \\
\hline GA & USGS-GAWSC & 11J012 & 311802084192302 & - & Mitchell & 311803 & 841923 & $5 / 23 / 2010$ & 120 & \\
\hline GA & USGS-GAWSC & $11 \mathrm{~K} 003$ & 312919084153801 & - & Dougherty & 312914 & 841530 & $5 / 23 / 2010$ & 172 & \\
\hline GA & USGS-GAWSC & $11 \mathrm{~K} 015$ & 312709084161701 & - & Dougherty & 312657 & 841603 & $5 / 20 / 2010$ & 169 & \\
\hline GA & USGS-GAWSC & $11 \mathrm{~K} 016$ & 312418084210001 & - & Baker & 312419 & 842060 & $5 / 20 / 2010$ & 149 & \\
\hline GA & USGS-GAWSC & $11 \mathrm{~K} 033$ & 312654084210104 & - & Dougherty & 312655 & 842101 & $5 / 20 / 2010$ & 167 & \\
\hline GA & USGS-GAWSC & $11 \mathrm{~K} 043$ & 312913084192601 & - & Dougherty & 312914 & 841926 & $5 / 20 / 2010$ & 177 & \\
\hline GA & USGS-GAWSC & 11L092 & 313504084165701 & - & Dougherty & 313504 & 841656 & $5 / 20 / 2010$ & 190 & \\
\hline GA & USGS-GAWSC & 11L111 & 313340084220001 & - & Dougherty & 313341 & 842160 & $5 / 20 / 2010$ & 197 & \\
\hline GA & USGS-GAWSC & 11L112 & 313614084203401 & - & Dougherty & 313615 & 842034 & $5 / 20 / 2010$ & 196 & \\
\hline GA & USGS-GAWSC & $11 \mathrm{M} 017$ & 314210084151901 & - & Lee & 314211 & 841519 & $5 / 26 / 2010$ & 247 & \\
\hline GA & USGS-GAWSC & $11 \mathrm{M} 025$ & 313836084210401 & - & Terrell & 313837 & 842104 & $5 / 19 / 2010$ & 247 & \\
\hline GA & USGS-GAWSC & $11 \mathrm{M} 041$ & 314247084212901 & - & Terrell & 314250 & 842128 & $5 / 18 / 2010$ & 276 & \\
\hline GA & USGS-GAWSC & $11 \mathrm{~N} 011$ & 314731084180401 & - & Terrell & 314731 & 841804 & $5 / 18 / 2010$ & 279 & \\
\hline GA & USGS-GAWSC & 12D039 & 304456084104801 & - & Grady & 304456 & 841048 & $5 / 17 / 2010$ & 62 & \\
\hline GA & USGS-GAWSC & 12F036 & 305235084125101 & - & Grady & 305236 & 841252 & $5 / 23 / 2010$ & 67 & \\
\hline GA & USGS-GAWSC & $12 \mathrm{H} 008$ & 311328084130701 & - & Mitchell & 311328 & 841255 & $6 / 3 / 2010$ & 126 & \\
\hline GA & USGS-GAWSC & 12J002 & 311909084111501 & - & Mitchell & 311910 & 841115 & $6 / 3 / 2010$ & 133 & \\
\hline GA & USGS-GAWSC & $12 \mathrm{~K} 014$ & 312617084110701 & - & Baker & 312618 & 841106 & $5 / 23 / 2010$ & 139 & \\
\hline GA & USGS-GAWSC & $12 \mathrm{~K} 141$ & 312950084131801 & - & Dougherty & 312951 & 841317 & $5 / 23 / 2010$ & 170 & \\
\hline
\end{tabular}


Table 1. Water-level measurements in Florida and parts of Georgia, South Carolina, and Alabama, May-June 2010.—Continued

[NGVD 29, National Geodetic Vertical Datum of 1929. Abbreviations for agency database: ACEPD, Alachua County Environmental Protection Department; FGS, Florida Geological Survey; GSA, Geological Survey of Alabama; NWFWMD, Northwest Florida Water Management District; SFWMD, South Florida Water Management District; SRWMD, Suwannee River Water Management District; USGSALWSC, U.S. Geological Survey Alabama Water Science Center; USGS-FLWSC, U.S. Geological Survey Florida Water Science Center; GAWSC, U.S. Geological Survey Georgia Water Science Center; SCWSC, U.S. Geological Survey South Carolina Water Science Center. Abbreviations for Station names: BLVD., boulevard; CO, county; CR, county road; E, east; HS, high school; IFAS, Institute of Food and Agricultural Services; LK, lake; MW, monitoring well; N, north; NE, northeast; NE, northeast; NF, national forest; NW, northwest; PK, park; R., river; RD., road; SE, southeast; SE, southeast; SR, state road; ST., street; TWR, tower; UFA, Upper Floridan aquifer; - - not available; \#, number; “, inches]

\begin{tabular}{|c|c|c|c|c|c|c|c|c|c|c|}
\hline State & $\begin{array}{c}\text { Agency } \\
\text { database }\end{array}$ & Station name & $\begin{array}{c}\text { USGS site } \\
\text { identification } \\
\text { number }\end{array}$ & $\begin{array}{l}\text { Local agency } \\
\text { unique site } \\
\text { identifier }\end{array}$ & County & $\begin{array}{l}\text { Latitude } \\
\text { (degree } \\
\text { minutes } \\
\text { and } \\
\text { seconds) } \\
\text { [DDMMS] }\end{array}$ & $\begin{array}{l}\text { Longitude } \\
\text { (degree } \\
\text { minutes } \\
\text { and } \\
\text { seconds) } \\
\text { ] [DDMMSS] }\end{array}$ & $\begin{array}{c}\text { Date of } \\
\text { measure- } \\
\text { ment }\end{array}$ & $\begin{array}{c}\text { Water } \\
\text { level } \\
\text { altitude } \\
\text { (feet } \\
\text { above } \\
\text { NGVD 29) }\end{array}$ & Remarks \\
\hline GA & USGS-GAWSC & $12 \mathrm{~K} 180$ & 312947084092201 & - & Dougherty & 312947 & 840922 & $5 / 23 / 2010$ & 151 & \\
\hline GA & USGS-GAWSC & 12L029 & 313450084091801 & - & Dougherty & 313451 & 840918 & $5 / 23 / 2010$ & 160 & \\
\hline GA & USGS-GAWSC & 12L030 & 313130084101001 & - & Dougherty & 313134 & 841010 & $5 / 23 / 2010$ & 161 & \\
\hline GA & USGS-GAWSC & 12L277 & 313040084125901 & - & Dougherty & 313042 & 841233 & $5 / 23 / 2010$ & 169 & \\
\hline GA & USGS-GAWSC & 12L311 & 313202084143801 & - & Dougherty & 313202 & 841438 & $5 / 20 / 2010$ & 173 & \\
\hline GA & USGS-GAWSC & $12 \mathrm{~L} 370$ & 313019084104601 & - & Dougherty & 313019 & 841046 & $5 / 23 / 2010$ & 155 & \\
\hline GA & USGS-GAWSC & 12L373 & 313000084100301 & - & Dougherty & 313001 & 841003 & $5 / 23 / 2010$ & 153 & \\
\hline GA & USGS-GAWSC & $12 \mathrm{M} 011$ & 314241084103701 & - & Lee & 314242 & 841037 & $5 / 25 / 2010$ & 206 & \\
\hline GA & USGS-GAWSC & $12 \mathrm{M} 017$ & 313808084093601 & - & Lee & 313809 & 840936 & $5 / 23 / 2010$ & 199 & \\
\hline GA & USGS-GAWSC & $12 \mathrm{M} 027$ & 314153084131101 & - & Lee & 314154 & 841311 & $5 / 25 / 2010$ & 182 & \\
\hline GA & USGS-GAWSC & $12 \mathrm{M} 060$ & 313919084113801 & - & Lee & 313919 & 841138 & $5 / 26 / 2010$ & 178 & \\
\hline GA & USGS-GAWSC & $12 \mathrm{~N} 004$ & 315228084100601 & - & Lee & 315229 & 841005 & $5 / 26 / 2010$ & 274 & \\
\hline GA & USGS-GAWSC & 13F034 & 305436084061901 & - & Grady & 305436 & 840619 & $5 / 17 / 2010$ & 87 & \\
\hline GA & USGS-GAWSC & $13 \mathrm{~F} 035$ & 305943084065001 & - & Thomas & 305943 & 840650 & $5 / 24 / 2010$ & 86 & \\
\hline GA & USGS-GAWSC & 13G006 & 310432084053001 & - & Thomas & 310417 & 840531 & $5 / 19 / 2010$ & 116 & \\
\hline GA & USGS-GAWSC & $13 \mathrm{H} 012$ & 311204084020901 & - & Mitchell & 311204 & 840209 & $5 / 26 / 2010$ & 132 & \\
\hline GA & USGS-GAWSC & 13J001 & 311743084023001 & - & Mitchell & 311730 & 840218 & $6 / 3 / 2010$ & 159 & \\
\hline GA & USGS-GAWSC & 13J004 & 312127084065801 & - & Mitchell & 312130 & 840657 & $5 / 23 / 2010$ & 147 & \\
\hline GA & USGS-GAWSC & 13J014 & 312023084001001 & - & Mitchell & 312023 & 840010 & $5 / 26 / 2010$ & 182 & \\
\hline GA & USGS-GAWSC & 13J015 & 311700084001801 & - & Mitchell & 311700 & 840018 & $5 / 26 / 2010$ & 157 & \\
\hline GA & USGS-GAWSC & $13 \mathrm{~K} 014$ & 312704084071601 & - & Dougherty & 312705 & 840716 & $5 / 23 / 2010$ & 151 & \\
\hline GA & USGS-GAWSC & $13 \mathrm{~K} 022$ & 312531084002201 & - & Mitchell & 312532 & 840022 & $6 / 3 / 2010$ & 180 & \\
\hline GA & USGS-GAWSC & 13L012 & 313105084064302 & - & Dougherty & 313106 & 840643 & $5 / 23 / 2010$ & 153 & \\
\hline GA & USGS-GAWSC & 13L047 & 313640084002101 & - & Dougherty & 313641 & 840021 & $5 / 20 / 2010$ & 198 & \\
\hline
\end{tabular}


Table 1. Water-level measurements in Florida and parts of Georgia, South Carolina, and Alabama, May-June 2010.—Continued

[NGVD 29, National Geodetic Vertical Datum of 1929. Abbreviations for agency database: ACEPD, Alachua County Environmental Protection Department; FGS, Florida Geological Survey; GSA, Geological Survey of Alabama; NWFWMD, Northwest Florida Water Management District; SFWMD, South Florida Water Management District; SRWMD, Suwannee River Water Management District; USGSALWSC, U.S. Geological Survey Alabama Water Science Center; USGS-FLWSC, U.S. Geological Survey Florida Water Science Center; GAWSC, U.S. Geological Survey Georgia Water Science Center; SCWSC, U.S. Geological Survey South Carolina Water Science Center. Abbreviations for Station names: BLVD., boulevard; CO, county; CR, county road; E, east; HS, high school; IFAS, Institute of Food and Agricultural Services; LK, lake; MW, monitoring well; N, north; NE, northeast; NE, northeast; NF, national forest; NW, northwest; PK, park; R., river; RD., road; SE, southeast; SE, southeast; SR, state road; ST., street; TWR, tower; UFA, Upper Floridan aquifer; - , not available; \#, number; “, inches]

\begin{tabular}{|c|c|c|c|c|c|c|c|c|c|c|}
\hline State & $\begin{array}{c}\text { Agency } \\
\text { database }\end{array}$ & Station name & $\begin{array}{c}\text { USGS site } \\
\text { identification } \\
\text { number }\end{array}$ & $\begin{array}{l}\text { Local agency } \\
\text { unique site } \\
\text { identifier }\end{array}$ & County & $\begin{array}{l}\text { Latitude } \\
\text { (degree } \\
\text { minutes } \\
\text { and } \\
\text { seconds) } \\
\text { [DDMMSS] }\end{array}$ & $\begin{array}{l}\text { Longitude } \\
\text { (degree } \\
\text { minutes } \\
\text { and } \\
\text { seconds) } \\
\text { ] [DDMMSS] }\end{array}$ & $\begin{array}{c}\text { Date of } \\
\text { measure- } \\
\text { ment }\end{array}$ & $\begin{array}{c}\text { Water } \\
\text { level } \\
\text { altitude } \\
\text { (feet } \\
\text { above } \\
\text { NGVD 29) }\end{array}$ & Remarks \\
\hline GA & USGS-GAWSC & 13L049 & 313521084051001 & - & Dougherty & 313522 & 840510 & $5 / 23 / 2010$ & 178 & \\
\hline GA & USGS-GAWSC & 13L180 & 313247084005001 & - & Dougherty & 313248 & 840050 & $5 / 23 / 2010$ & 184 & \\
\hline GA & USGS-GAWSC & $13 \mathrm{M} 006$ & 314330084005402 & - & Worth & 314331 & 840051 & $5 / 23 / 2010$ & 226 & \\
\hline GA & USGS-GAWSC & $13 \mathrm{M} 027$ & 314252084060102 & - & Lee & 314253 & 840601 & $5 / 25 / 2010$ & 216 & \\
\hline GA & USGS-GAWSC & 13M056 & 314134084013801 & - & Lee & 314135 & 840138 & $5 / 25 / 2010$ & 232 & \\
\hline GA & USGS-GAWSC & $13 \mathrm{M} 086$ & 314442084034501 & - & Lee & 314443 & 840345 & $5 / 25 / 2010$ & 226 & \\
\hline GA & USGS-GAWSC & $13 \mathrm{~N} 003$ & 314809084071901 & - & Lee & 314810 & 840719 & $5 / 25 / 2010$ & 253 & \\
\hline GA & USGS-GAWSC & 13N009 & 315205084030501 & - & Lee & 315206 & 840305 & $5 / 26 / 2010$ & 293 & \\
\hline GA & USGS-GAWSC & 13N014 & 314932084032401 & - & Lee & 314932 & 840324 & $5 / 25 / 2010$ & 268 & \\
\hline GA & USGS-GAWSC & 13Р019 & 315908084013901 & - & Sumter & 315908 & 840139 & $5 / 20 / 2010$ & 283 & \\
\hline GA & USGS-GAWSC & 14D004 & 304126083533901 & - & Thomas & 304126 & 835339 & $5 / 24 / 2010$ & 64 & \\
\hline GA & USGS-GAWSC & 14E048 & 304652083553601 & - & Thomas & 304652 & 835536 & $5 / 24 / 2010$ & 60 & \\
\hline GA & USGS-GAWSC & 14F006 & 305932083540001 & - & Thomas & 305933 & 835404 & $5 / 19 / 2010$ & 69 & \\
\hline GA & USGS-GAWSC & $14 \mathrm{~F} 010$ & 305238083591301 & - & Thomas & 305251 & 835903 & $5 / 19 / 2010$ & 78 & \\
\hline GA & USGS-GAWSC & 14F013 & 305959083580401 & - & Thomas & 305940 & 835813 & $5 / 19 / 2010$ & 87 & \\
\hline GA & USGS-GAWSC & 14G010 & 310440083534501 & - & Colquitt & 310445 & 835342 & $5 / 21 / 2010$ & 73 & \\
\hline GA & USGS-GAWSC & $14 \mathrm{H} 007$ & 311400083544001 & - & Colquitt & 311401 & 835440 & $5 / 21 / 2010$ & 151 & \\
\hline GA & USGS-GAWSC & $14 \mathrm{H} 009$ & 310815083572401 & - & Colquitt & 310813 & 835729 & $5 / 21 / 2010$ & 94 & \\
\hline GA & USGS-GAWSC & 14J018 & 312100083573401 & - & Worth & 312101 & 835734 & $5 / 27 / 2010$ & 170 & \\
\hline GA & USGS-GAWSC & 14J019 & 312157083530301 & - & Worth & 312158 & 835303 & $5 / 27 / 2010$ & 186 & \\
\hline GA & USGS-GAWSC & 14J021 & 312005083555301 & - & Worth & 312006 & 835553 & $5 / 27 / 2010$ & 181 & \\
\hline GA & USGS-GAWSC & 14J022 & 312223083560301 & - & Worth & 312224 & 835603 & $5 / 25 / 2010$ & 218 & \\
\hline GA & USGS-GAWSC & $14 \mathrm{~K} 013$ & 312550083552901 & - & Worth & 312551 & 835529 & $5 / 27 / 2010$ & 208 & \\
\hline GA & USGS-GAWSC & 14L006 & 313400083555801 & - & Worth & 313401 & 835558 & $5 / 24 / 2010$ & 225 & \\
\hline
\end{tabular}


Table 1. Water-level measurements in Florida and parts of Georgia, South Carolina, and Alabama, May-June 2010.—Continued

[NGVD 29, National Geodetic Vertical Datum of 1929. Abbreviations for agency database: ACEPD, Alachua County Environmental Protection Department; FGS, Florida Geological Survey; GSA, Geological Survey of Alabama; NWFWMD, Northwest Florida Water Management District; SFWMD, South Florida Water Management District; SRWMD, Suwannee River Water Management District; USGSALWSC, U.S. Geological Survey Alabama Water Science Center; USGS-FLWSC, U.S. Geological Survey Florida Water Science Center; GAWSC, U.S. Geological Survey Georgia Water Science Center; SCWSC, U.S. Geological Survey South Carolina Water Science Center. Abbreviations for Station names: BLVD., boulevard; CO, county; CR, county road; E, east; HS, high school; IFAS, Institute of Food and Agricultural Services; LK, lake; MW, monitoring well; N, north; NE, northeast; NE, northeast; NF, national forest; NW, northwest; PK, park; R., river; RD., road; SE, southeast; SE, southeast; SR, state road; ST., street; TWR, tower; UFA, Upper Floridan aquifer; - - not available; \#, number; “, inches]

\begin{tabular}{|c|c|c|c|c|c|c|c|c|c|c|}
\hline State & $\begin{array}{c}\text { Agency } \\
\text { database }\end{array}$ & Station name & $\begin{array}{c}\text { USGS site } \\
\text { identification } \\
\text { number }\end{array}$ & $\begin{array}{l}\text { Local agency } \\
\text { unique site } \\
\text { identifier }\end{array}$ & County & $\begin{array}{l}\text { Latitude } \\
\text { (degree } \\
\text { minutes } \\
\text { and } \\
\text { seconds) } \\
\text { [DDMMS] }\end{array}$ & $\begin{array}{l}\text { Longitude } \\
\text { (degree } \\
\text { minutes } \\
\text { and } \\
\text { seconds) } \\
\text { ] [DDMMSS] }\end{array}$ & $\begin{array}{c}\text { Date of } \\
\text { measure- } \\
\text { ment }\end{array}$ & $\begin{array}{c}\text { Water } \\
\text { level } \\
\text { altitude } \\
\text { (feet } \\
\text { above } \\
\text { NGVD 29) }\end{array}$ & Remarks \\
\hline GA & USGS-GAWSC & 14L011 & 313503083585201 & - & Worth & 313504 & 835852 & $5 / 26 / 2010$ & 218 & \\
\hline GA & USGS-GAWSC & 14L013 & 313027083570901 & - & Worth & 313028 & 835709 & $5 / 26 / 2010$ & 212 & \\
\hline GA & USGS-GAWSC & 14L014 & 313729083550301 & - & Worth & 313730 & 835503 & $5 / 24 / 2010$ & 240 & \\
\hline GA & USGS-GAWSC & 14M006 & 314336083572801 & - & Worth & 314337 & 835728 & $5 / 26 / 2010$ & 234 & \\
\hline GA & USGS-GAWSC & 14N012 & 315024083550801 & - & Worth & 315024 & 835508 & $5 / 21 / 2010$ & 255 & \\
\hline GA & USGS-GAWSC & 15E002 & 305002083481901 & - & Thomas & 305003 & 834823 & $5 / 19 / 2010$ & 68 & \\
\hline GA & USGS-GAWSC & $15 \mathrm{E} 015$ & 304504083485301 & - & Thomas & 304505 & 834853 & $5 / 19 / 2010$ & 70 & \\
\hline GA & USGS-GAWSC & $15 \mathrm{~F} 003$ & 305600083472901 & - & Thomas & 305601 & 834729 & $5 / 19 / 2010$ & 68 & \\
\hline GA & USGS-GAWSC & 15G022 & 310720083505801 & - & Colquitt & 310717 & 835058 & $5 / 21 / 2010$ & 77 & \\
\hline GA & USGS-GAWSC & $15 \mathrm{H} 022$ & 311427083464201 & - & Colquitt & 311428 & 834633 & $5 / 21 / 2010$ & 96 & \\
\hline GA & USGS-GAWSC & $15 \mathrm{~J} 013$ & 311643083490001 & - & Colquitt & 311644 & 834901 & $5 / 21 / 2010$ & 167 & \\
\hline GA & USGS-GAWSC & 15L020 & 313146083491601 & - & Worth & 313147 & 834916 & $5 / 23 / 2010$ & 204 & \\
\hline GA & USGS-GAWSC & 15L052 & 313010083504401 & - & Worth & 313010 & 835044 & $5 / 27 / 2010$ & 219 & \\
\hline GA & USGS-GAWSC & $15 \mathrm{M} 004$ & 314123083495801 & - & Worth & 314124 & 834958 & $5 / 20 / 2010$ & 260 & \\
\hline GA & USGS-GAWSC & $15 \mathrm{M} 005$ & 313909083491201 & - & Worth & 313910 & 834912 & $5 / 19 / 2010$ & 252 & \\
\hline GA & USGS-GAWSC & $15 \mathrm{M} 013$ & 313947083454701 & - & Turner & 313947 & 834547 & $5 / 19 / 2010$ & 244 & \\
\hline GA & USGS-GAWSC & $15 \mathrm{P} 018$ & 315703083493601 & - & Crisp & 315705 & 834936 & $5 / 20 / 2010$ & 276 & \\
\hline GA & USGS-GAWSC & 15Q011 & 320021083473401 & - & Crisp & 320034 & 834731 & $5 / 17 / 2010$ & 298 & \\
\hline GA & USGS-GAWSC & 15Q016 & 320139083511602 & - & Crisp & 320148 & 835108 & $5 / 23 / 2010$ & 280 & \\
\hline GA & USGS-GAWSC & $15 \mathrm{~S} 004$ & 324715083452801 & - & Houston & 322029 & 834714 & $5 / 26 / 2010$ & 458 & \\
\hline GA & USGS-GAWSC & $15 \mathrm{~S} 007$ & 321523083490001 & - & Dooly & 321523 & 834900 & $5 / 26 / 2010$ & 325 & \\
\hline GA & USGS-GAWSC & $16 \mathrm{D} 006$ & 304446083400401 & - & Brooks & 304432 & 833946 & $5 / 26 / 2010$ & 81 & \\
\hline GA & USGS-GAWSC & 16E001 & 304956083430201 & - & Brooks & 304955 & 834302 & $5 / 19 / 2010$ & 70 & \\
\hline GA & USGS-GAWSC & 16F005 & 305512083375101 & - & Brooks & 305513 & 833751 & $5 / 26 / 2010$ & 73 & \\
\hline
\end{tabular}


Table 1. Water-level measurements in Florida and parts of Georgia, South Carolina, and Alabama, May-June 2010.—Continued

[NGVD 29, National Geodetic Vertical Datum of 1929. Abbreviations for agency database: ACEPD, Alachua County Environmental Protection Department; FGS, Florida Geological Survey; GSA, Geological Survey of Alabama; NWFWMD, Northwest Florida Water Management District; SFWMD, South Florida Water Management District; SRWMD, Suwannee River Water Management District; USGSALWSC, U.S. Geological Survey Alabama Water Science Center; USGS-FLWSC, U.S. Geological Survey Florida Water Science Center; GAWSC, U.S. Geological Survey Georgia Water Science Center; SCWSC, U.S. Geological Survey South Carolina Water Science Center. Abbreviations for Station names: BLVD., boulevard; CO, county; CR, county road; E, east; HS, high school; IFAS, Institute of Food and Agricultural Services; LK, lake; MW, monitoring well; N, north; NE, northeast; NE, northeast; NF, national forest; NW, northwest; PK, park; R., river; RD., road; SE, southeast; SE, southeast; SR, state road; ST., street; TWR, tower; UFA, Upper Floridan aquifer; —, not available; \#, number; “, inches]

\begin{tabular}{|c|c|c|c|c|c|c|c|c|c|c|}
\hline State & $\begin{array}{c}\text { Agency } \\
\text { database }\end{array}$ & Station name & $\begin{array}{c}\text { USGS site } \\
\text { identification } \\
\text { number }\end{array}$ & $\begin{array}{l}\text { Local agency } \\
\text { unique site } \\
\text { identifier }\end{array}$ & County & $\begin{array}{l}\text { Latitude } \\
\text { (degree } \\
\text { minutes } \\
\text { and } \\
\text { seconds) } \\
\text { [DDMMSS] }\end{array}$ & $\begin{array}{l}\text { Longitude } \\
\text { (degree } \\
\text { minutes } \\
\text { and } \\
\text { seconds) } \\
\text { [DDMMSS] }\end{array}$ & $\begin{array}{c}\text { Date of } \\
\text { measure- } \\
\text { ment }\end{array}$ & $\begin{array}{c}\text { Water } \\
\text { level } \\
\text { altitude } \\
\text { (feet } \\
\text { above } \\
\text { NGVD 29) }\end{array}$ & Remarks \\
\hline GA & USGS-GAWSC & 16G001 & 310211083405301 & - & Colquitt & 310311 & 834106 & $5 / 27 / 2010$ & 75 & \\
\hline GA & USGS-GAWSC & 16G009 & 310106083432301 & - & Brooks & 310104 & 834323 & $5 / 19 / 2010$ & 73 & \\
\hline GA & USGS-GAWSC & $16 \mathrm{H} 028$ & 311006083402001 & - & Colquitt & 311012 & 834012 & $5 / 27 / 2010$ & 72 & \\
\hline GA & USGS-GAWSC & $16 \mathrm{H} 037$ & 311452083400701 & - & Colquitt & 311457 & 834010 & $5 / 26 / 2010$ & 63 & \\
\hline GA & USGS-GAWSC & 16J009 & 311930083390901 & - & Colquitt & 311933 & 833929 & $5 / 26 / 2010$ & 120 & \\
\hline GA & USGS-GAWSC & 16J019 & 311730083412501 & - & Colquitt & 311734 & 834128 & $5 / 26 / 2010$ & 116 & \\
\hline GA & USGS-GAWSC & 16J040 & 312000083375901 & - & Tift & 311960 & 833759 & $5 / 27 / 2010$ & 93 & \\
\hline GA & USGS-GAWSC & $16 \mathrm{~K} 013$ & 312529083423501 & - & Worth & 312527 & 834235 & $5 / 27 / 2010$ & 181 & \\
\hline GA & USGS-GAWSC & 16L037 & 313525083374801 & - & Tift & 313525 & 833748 & $5 / 27 / 2010$ & 195 & \\
\hline GA & USGS-GAWSC & 16L048 & 313700083410201 & - & Turner & 313700 & 834102 & $5 / 19 / 2010$ & 195 & \\
\hline GA & USGS-GAWSC & 16L049 & 313037083435701 & - & Worth & 313037 & 834357 & $5 / 27 / 2010$ & 202 & \\
\hline GA & USGS-GAWSC & $16 \mathrm{M} 027$ & 313819083432801 & - & Turner & 313819 & 834328 & $5 / 19 / 2010$ & 207 & \\
\hline GA & USGS-GAWSC & $16 \mathrm{M} 047$ & 314125083384201 & - & Turner & 314125 & 833842 & $5 / 18 / 2010$ & 197 & \\
\hline GA & USGS-GAWSC & $16 \mathrm{~N} 013$ & 314618083412702 & - & Turner & 314618 & 834127 & $5 / 18 / 2010$ & 279 & \\
\hline GA & USGS-GAWSC & 16Р022 & 315610083392301 & - & Crisp & 315610 & 833923 & $5 / 18 / 2010$ & 257 & \\
\hline GA & USGS-GAWSC & 16Q022 & 320720083373001 & - & Dooly & 320720 & 833730 & $5 / 26 / 2010$ & 319 & \\
\hline GA & USGS-GAWSC & 16Q023 & 320704083424101 & - & Dooly & 320704 & 834241 & $5 / 26 / 2010$ & 326 & \\
\hline GA & USGS-GAWSC & 17D007 & 304033083310401 & - & Brooks & 304034 & 833104 & $5 / 26 / 2010$ & 87 & \\
\hline GA & USGS-GAWSC & 17E006 & 304510083334801 & - & Brooks & 304508 & 833347 & $5 / 26 / 2010$ & 79 & \\
\hline GA & USGS-GAWSC & 17E031 & 305112083304301 & - & Brooks & 305113 & 833043 & $5 / 25 / 2010$ & 92 & \\
\hline GA & USGS-GAWSC & 17G017 & 310714083324001 & - & Cook & 310715 & 833240 & $5 / 26 / 2010$ & 59 & \\
\hline GA & USGS-GAWSC & $17 \mathrm{H} 007$ & 310931083314401 & - & Cook & 310932 & 833144 & $5 / 26 / 2010$ & 62 & \\
\hline GA & USGS-GAWSC & $17 \mathrm{H} 027$ & 311220083330201 & - & Colquitt & 311223 & 833303 & $5 / 27 / 2010$ & 63 & \\
\hline GA & USGS-GAWSC & 17J009 & 311845083313901 & - & Colquitt & 311843 & 833128 & $5 / 26 / 2010$ & 76 & \\
\hline
\end{tabular}


Table 1. Water-level measurements in Florida and parts of Georgia, South Carolina, and Alabama, May-June 2010.—Continued

[NGVD 29, National Geodetic Vertical Datum of 1929. Abbreviations for agency database: ACEPD, Alachua County Environmental Protection Department; FGS, Florida Geological Survey; GSA, Geological Survey of Alabama; NWFWMD, Northwest Florida Water Management District; SFWMD, South Florida Water Management District; SRWMD, Suwannee River Water Management District; USGSALWSC, U.S. Geological Survey Alabama Water Science Center; USGS-FLWSC, U.S. Geological Survey Florida Water Science Center; GAWSC, U.S. Geological Survey Georgia Water Science Center; SCWSC, U.S. Geological Survey South Carolina Water Science Center. Abbreviations for Station names: BLVD., boulevard; CO, county; CR, county road; E, east; HS, high school; IFAS, Institute of Food and Agricultural Services; LK, lake; MW, monitoring well; N, north; NE, northeast; NE, northeast; NF, national forest; NW, northwest; PK, park; R., river; RD., road; SE, southeast; SE, southeast; SR, state road; ST., street; TWR, tower; UFA, Upper Floridan aquifer; - - not available; \#, number; “, inches]

\begin{tabular}{|c|c|c|c|c|c|c|c|c|c|c|}
\hline State & $\begin{array}{c}\text { Agency } \\
\text { database }\end{array}$ & Station name & $\begin{array}{c}\text { USGS site } \\
\text { identification } \\
\text { number }\end{array}$ & $\begin{array}{l}\text { Local agency } \\
\text { unique site } \\
\text { identifier }\end{array}$ & County & $\begin{array}{c}\text { Latitude } \\
\text { (degree } \\
\text { minutes } \\
\text { and } \\
\text { seconds) } \\
\text { [DDMMSS] }\end{array}$ & $\begin{array}{l}\text { Longitude } \\
\text { (degree } \\
\text { minutes } \\
\text { and } \\
\text { seconds) } \\
\text { ] [DDMMSS] }\end{array}$ & $\begin{array}{c}\text { Date of } \\
\text { measure- } \\
\text { ment }\end{array}$ & $\begin{array}{c}\text { Water } \\
\text { level } \\
\text { altitude } \\
\text { (feet } \\
\text { above } \\
\text { NGVD 29) }\end{array}$ & Remarks \\
\hline GA & USGS-GAWSC & 17J027 & 311531083303901 & - & Cook & 311531 & 833039 & $5 / 26 / 2010$ & 59 & \\
\hline GA & USGS-GAWSC & $17 \mathrm{~K} 052$ & 312949083303201 & - & Tift & 312950 & 833037 & $5 / 27 / 2010$ & 195 & \\
\hline GA & USGS-GAWSC & $17 \mathrm{~K} 112$ & 312310083311202 & - & Tift & 312310 & 833112 & $5 / 27 / 2010$ & 187 & \\
\hline GA & USGS-GAWSC & 17L009 & 313640083321201 & - & Turner & 313645 & 833213 & $5 / 18 / 2010$ & 193 & \\
\hline GA & USGS-GAWSC & 17L014 & 313004083371801 & - & Tift & 313005 & 833718 & $5 / 25 / 2010$ & 197 & \\
\hline GA & USGS-GAWSC & 17L042 & 313349083353601 & - & Tift & 313349 & 833536 & $5 / 27 / 2010$ & 213 & \\
\hline GA & USGS-GAWSC & $17 \mathrm{M} 007$ & 314254083342001 & - & Turner & 314253 & 833417 & $5 / 18 / 2010$ & 210 & \\
\hline GA & USGS-GAWSC & $17 \mathrm{M} 035$ & 314302083361101 & - & Turner & 314302 & 833611 & $5 / 18 / 2010$ & 208 & \\
\hline GA & USGS-GAWSC & 17N011 & 314716083311401 & - & Turner & 314716 & 833114 & $5 / 19 / 2010$ & 209 & \\
\hline GA & USGS-GAWSC & 17N013 & 314700083330501 & - & Turner & 314660 & 833305 & $5 / 19 / 2010$ & 216 & \\
\hline GA & USGS-GAWSC & 17P014 & 315458083361401 & - & Wilcox & 315458 & 833614 & $6 / 3 / 2010$ & 244 & \\
\hline GA & USGS-GAWSC & 17S012 & 321851083300901 & - & Pulaski & 321851 & 833009 & $5 / 26 / 2010$ & 229 & \\
\hline GA & USGS-GAWSC & $18 \mathrm{D} 003$ & 303828083242201 & - & Brooks & 303829 & 832422 & $5 / 26 / 2010$ & 68 & \\
\hline GA & USGS-GAWSC & $18 \mathrm{E} 005$ & 304725083241801 & - & Lowndes & 304726 & 832418 & $5 / 26 / 2010$ & 89 & \\
\hline GA & USGS-GAWSC & $18 \mathrm{~F} 001$ & 305626083240101 & - & Lowndes & 305627 & 832401 & $5 / 26 / 2010$ & 100 & \\
\hline GA & USGS-GAWSC & $18 \mathrm{~F} 013$ & 305949083261401 & - & Lowndes & 305950 & 832614 & $5 / 26 / 2010$ & 98 & \\
\hline GA & USGS-GAWSC & $18 \mathrm{H} 016$ & 310813083260301 & - & Cook & 310814 & 832603 & $5 / 23 / 2010$ & 62 & \\
\hline GA & USGS-GAWSC & $18 \mathrm{H} 042$ & 311255083275801 & - & Cook & 311256 & 832758 & $5 / 26 / 2010$ & 62 & \\
\hline GA & USGS-GAWSC & 18J041 & 311949083282701 & - & Tift & 311949 & 832827 & $5 / 26 / 2010$ & 83 & \\
\hline GA & USGS-GAWSC & 18J051 & 311805083251401 & - & Berrien & 311805 & 832514 & $5 / 26 / 2010$ & 60 & \\
\hline GA & USGS-GAWSC & $18 \mathrm{~K} 049$ & 312712082593301 & - & Tift & 312713 & 832933 & $5 / 23 / 2010$ & 197 & \\
\hline GA & USGS-GAWSC & $18 \mathrm{M} 017$ & 313836083243801 & - & Irwin & 313836 & 832438 & $5 / 25 / 2010$ & 201 & \\
\hline GA & USGS-GAWSC & $18 \mathrm{~N} 003$ & 314745083261401 & - & Ben Hill & 314749 & 832606 & $5 / 28 / 2010$ & 202 & \\
\hline GA & USGS-GAWSC & 18Q005 & 320052083291901 & - & Wilcox & 320052 & 832919 & $5 / 24 / 2010$ & 239 & \\
\hline
\end{tabular}


Table 1. Water-level measurements in Florida and parts of Georgia, South Carolina, and Alabama, May-June 2010.—Continued

[NGVD 29, National Geodetic Vertical Datum of 1929. Abbreviations for agency database: ACEPD, Alachua County Environmental Protection Department; FGS, Florida Geological Survey; GSA, Geological Survey of Alabama; NWFWMD, Northwest Florida Water Management District; SFWMD, South Florida Water Management District; SRWMD, Suwannee River Water Management District; USGSALWSC, U.S. Geological Survey Alabama Water Science Center; USGS-FLWSC, U.S. Geological Survey Florida Water Science Center; GAWSC, U.S. Geological Survey Georgia Water Science Center; SCWSC, U.S. Geological Survey South Carolina Water Science Center. Abbreviations for Station names: BLVD., boulevard; CO, county; CR, county road; E, east; HS, high school; IFAS, Institute of Food and Agricultural Services; LK, lake; MW, monitoring well; N, north; NE, northeast; NE, northeast; NF, national forest; NW, northwest; PK, park; R., river; RD., road; SE, southeast; SE, southeast; SR, state road; ST., street; TWR, tower; UFA, Upper Floridan aquifer; - , not available; \#, number; “, inches]

\begin{tabular}{|c|c|c|c|c|c|c|c|c|c|c|}
\hline State & $\begin{array}{c}\text { Agency } \\
\text { database }\end{array}$ & Station name & $\begin{array}{c}\text { USGS site } \\
\text { identification } \\
\text { number }\end{array}$ & $\begin{array}{l}\text { Local agency } \\
\text { unique site } \\
\text { identifier }\end{array}$ & County & $\begin{array}{l}\text { Latitude } \\
\text { (degree } \\
\text { minutes } \\
\text { and } \\
\text { seconds) } \\
\text { [DDMMSS] }\end{array}$ & $\begin{array}{l}\text { Longitude } \\
\text { (degree } \\
\text { minutes } \\
\text { and } \\
\text { seconds) } \\
\text { ] [DDMMSS] }\end{array}$ & $\begin{array}{c}\text { Date of } \\
\text { measure- } \\
\text { ment }\end{array}$ & $\begin{array}{c}\text { Water } \\
\text { level } \\
\text { altitude } \\
\text { (feet } \\
\text { above } \\
\text { NGVD 29) }\end{array}$ & Remarks \\
\hline GA & USGS-GAWSC & $18 \mathrm{~S} 001$ & 322005083244001 & - & Bleckley & 322003 & 832440 & $5 / 20 / 2010$ & 268 & \\
\hline GA & USGS-GAWSC & 18U004 & 323549083282401 & - & Twiggs & 323550 & 832824 & $5 / 20 / 2010$ & 283 & \\
\hline GA & USGS-GAWSC & 19D010 & 303851083183001 & - & Lowndes & 303852 & 831830 & $5 / 26 / 2010$ & 65 & \\
\hline GA & USGS-GAWSC & 19D044 & 303805083164301 & - & Lowndes & 303806 & 831643 & $5 / 26 / 2010$ & 61 & \\
\hline GA & USGS-GAWSC & 19E009 & 304949083165301 & - & Lowndes & 304952 & 831658 & $5 / 23 / 2010$ & 90 & \\
\hline GA & USGS-GAWSC & 19E024 & 304625083180801 & - & Lowndes & 304626 & 831808 & $5 / 25 / 2010$ & 76 & \\
\hline GA & USGS-GAWSC & 19F034 & 305901083200001 & - & Lowndes & 305902 & 831960 & $5 / 26 / 2010$ & 89 & \\
\hline GA & USGS-GAWSC & 19F048 & 305257083183501 & - & Lowndes & 305260 & 831839 & $5 / 26 / 2010$ & 94 & \\
\hline GA & USGS-GAWSC & 19G011 & 310221083212201 & - & Cook & 310222 & 832122 & $5 / 26 / 2010$ & 68 & \\
\hline GA & USGS-GAWSC & 19H038 & 311028083204901 & - & Cook & 311028 & 832049 & $5 / 26 / 2010$ & 63 & \\
\hline GA & USGS-GAWSC & 19J011 & 311622083192401 & - & Berrien & 311622 & 831924 & $5 / 26 / 2010$ & 60 & \\
\hline GA & USGS-GAWSC & 19J013 & 312147083162101 & - & Berrien & 312147 & 831621 & $5 / 27 / 2010$ & 59 & \\
\hline GA & USGS-GAWSC & 19L026 & 313339083220601 & - & Irwin & 313339 & 832206 & $5 / 25 / 2010$ & 195 & \\
\hline GA & USGS-GAWSC & 19L029 & 313148083175001 & - & Irwin & 313148 & 831750 & $5 / 25 / 2010$ & 194 & \\
\hline GA & USGS-GAWSC & 19M002 & 314118083161101 & - & Ben Hill & 314119 & 831611 & $5 / 28 / 2010$ & 181 & \\
\hline GA & USGS-GAWSC & 19N003 & 314523083174801 & - & Ben Hill & 314524 & 831748 & $5 / 28 / 2010$ & 204 & \\
\hline GA & USGS-GAWSC & 19N007 & 314924083162001 & - & Ben Hill & 314924 & 831620 & $5 / 28 / 2010$ & 168 & \\
\hline GA & USGS-GAWSC & 19Q002 & 320646083192401 & - & Dodge & 320646 & 831924 & $6 / 3 / 2010$ & 188 & \\
\hline GA & USGS-GAWSC & 19Q004 & 320408083151002 & - & Dodge & 320408 & 831510 & $5 / 24 / 2010$ & 182 & \\
\hline GA & USGS-GAWSC & 19R004 & 321445083211501 & - & Dodge & 321445 & 832115 & $5 / 24 / 2010$ & 238 & \\
\hline GA & USGS-GAWSC & $19 \mathrm{~S} 003$ & 321833083192901 & - & Bleckley & 321834 & 831929 & $5 / 20 / 2010$ & 255 & \\
\hline GA & USGS-GAWSC & 19T016 & 322606083163301 & - & Bleckley & 322606 & 831633 & $5 / 20 / 2010$ & 272 & \\
\hline GA & USGS-GAWSC & 19U002 & 323015083180201 & - & Bleckley & 323016 & 831802 & $6 / 2 / 2010$ & 267 & \\
\hline GA & USGS-GAWSC & 19V007 & 323825083221201 & - & Twiggs & 323826 & 832212 & $5 / 20 / 2010$ & 477 & \\
\hline
\end{tabular}


Table 1. Water-level measurements in Florida and parts of Georgia, South Carolina, and Alabama, May-June 2010.—Continued

[NGVD 29, National Geodetic Vertical Datum of 1929. Abbreviations for agency database: ACEPD, Alachua County Environmental Protection Department; FGS, Florida Geological Survey; GSA, Geological Survey of Alabama; NWFWMD, Northwest Florida Water Management District; SFWMD, South Florida Water Management District; SRWMD, Suwannee River Water Management District; USGSALWSC, U.S. Geological Survey Alabama Water Science Center; USGS-FLWSC, U.S. Geological Survey Florida Water Science Center; GAWSC, U.S. Geological Survey Georgia Water Science Center; SCWSC, U.S. Geological Survey South Carolina Water Science Center. Abbreviations for Station names: BLVD., boulevard; CO, county; CR, county road; E, east; HS, high school; IFAS, Institute of Food and Agricultural Services; LK, lake; MW, monitoring well; N, north; NE, northeast; NE, northeast; NF, national forest; NW, northwest; PK, park; R., river; RD., road; SE, southeast; SE, southeast; SR, state road; ST., street; TWR, tower; UFA, Upper Floridan aquifer; - - not available; \#, number; “, inches]

\begin{tabular}{|c|c|c|c|c|c|c|c|c|c|c|}
\hline State & $\begin{array}{c}\text { Agency } \\
\text { database }\end{array}$ & Station name & $\begin{array}{c}\text { USGS site } \\
\text { identification } \\
\text { number }\end{array}$ & $\begin{array}{l}\text { Local agency } \\
\text { unique site } \\
\text { identifier }\end{array}$ & County & $\begin{array}{l}\text { Latitude } \\
\text { (degree } \\
\text { minutes } \\
\text { and } \\
\text { seconds) } \\
\text { [DDMMS] }\end{array}$ & $\begin{array}{l}\text { Longitude } \\
\text { (degree } \\
\text { minutes } \\
\text { and } \\
\text { seconds) } \\
\text { ] [DDMMSS] }\end{array}$ & $\begin{array}{c}\text { Date of } \\
\text { measure- } \\
\text { ment }\end{array}$ & $\begin{array}{c}\text { Water } \\
\text { level } \\
\text { altitude } \\
\text { (feet } \\
\text { above } \\
\text { NGVD 29) }\end{array}$ & Remarks \\
\hline GA & USGS-GAWSC & 20D018 & 304136083095901 & - & Lowndes & 304137 & 830959 & $5 / 20 / 2010$ & 74 & \\
\hline GA & USGS-GAWSC & 20D051 & 304447083112701 & - & Lowndes & 304447 & 831127 & $5 / 26 / 2010$ & 78 & \\
\hline GA & USGS-GAWSC & $20 \mathrm{~F} 027$ & 305310083125201 & - & Lowndes & 305309 & 831256 & $5 / 26 / 2010$ & 81 & \\
\hline GA & USGS-GAWSC & 20G034 & 310603083133302 & - & Berrien & 310603 & 831333 & $5 / 25 / 2010$ & 62 & \\
\hline GA & USGS-GAWSC & $20 \mathrm{H} 003$ & 311237083135201 & - & Berrien & 311235 & 831352 & $5 / 25 / 2010$ & 62 & \\
\hline GA & USGS-GAWSC & 20K019 & 312909083143701 & - & Irwin & 312909 & 831437 & $5 / 25 / 2010$ & 180 & \\
\hline GA & USGS-GAWSC & $20 \mathrm{~K} 020$ & 312810083104701 & - & Berrien & 312810 & 831047 & $5 / 27 / 2010$ & 125 & \\
\hline GA & USGS-GAWSC & 20L002 & 313333083103801 & - & Irwin & 313347 & 831049 & $5 / 25 / 2010$ & 182 & \\
\hline GA & USGS-GAWSC & 20L025 & 313405083143401 & - & Irwin & 313405 & 831434 & $5 / 25 / 2010$ & 199 & \\
\hline GA & USGS-GAWSC & $20 \mathrm{M} 007$ & 314242083100601 & - & Ben Hill & 314243 & 831006 & $5 / 28 / 2010$ & 172 & \\
\hline GA & USGS-GAWSC & 20N016 & 314513083104101 & - & Ben Hill & 314513 & 831041 & $5 / 28 / 2010$ & 161 & \\
\hline GA & USGS-GAWSC & 20N018 & 315144083080101 & - & Telfair & 315144 & 830801 & $5 / 28 / 2010$ & 157 & \\
\hline GA & USGS-GAWSC & 20Q001 & 320652083100201 & - & Dodge & 320652 & 831002 & $5 / 24 / 2010$ & 174 & \\
\hline GA & USGS-GAWSC & 20R002 & 321122083110001 & - & Dodge & 321123 & 831060 & $5 / 24 / 2010$ & 185 & \\
\hline GA & USGS-GAWSC & 20U008 & 323420083100901 & - & Laurens & 323421 & 831009 & $6 / 2 / 2010$ & 251 & \\
\hline GA & USGS-GAWSC & $21 \mathrm{D} 028$ & 304150083015802 & - & Echols & 304150 & 830158 & $5 / 25 / 2010$ & 52 & \\
\hline GA & USGS-GAWSC & 21E002 & 304941083032801 & - & Echols & 304945 & 830321 & $5 / 25 / 2010$ & 75 & \\
\hline GA & USGS-GAWSC & 21E012 & 304610083000502 & - & Echols & 304610 & 830005 & $5 / 25 / 2010$ & 58 & \\
\hline GA & USGS-GAWSC & 21F004 & 305448083044201 & - & Lowndes & 305449 & 830442 & $5 / 25 / 2010$ & 72 & \\
\hline GA & USGS-GAWSC & $21 \mathrm{H} 006$ & 310802083014701 & - & Lanier & 310802 & 830147 & $5 / 25 / 2010$ & 65 & \\
\hline GA & USGS-GAWSC & 21J009 & 311909083054301 & - & Berrien & 311909 & 830543 & $5 / 25 / 2010$ & 53 & \\
\hline GA & USGS-GAWSC & $21 \mathrm{~K} 003$ & 312506083014401 & - & Atkinson & 312506 & 830144 & $5 / 24 / 2010$ & 58 & \\
\hline GA & USGS-GAWSC & $21 \mathrm{M} 020$ & 314244083005201 & - & Ben Hill & 314244 & 830052 & $5 / 27 / 2010$ & 160 & \\
\hline GA & USGS-GAWSC & $21 \mathrm{~N} 009$ & 314631083054802 & - & Ben Hill & 314631 & 830548 & $5 / 28 / 2010$ & 161 & \\
\hline
\end{tabular}


Table 1. Water-level measurements in Florida and parts of Georgia, South Carolina, and Alabama, May-June 2010.—Continued

[NGVD 29, National Geodetic Vertical Datum of 1929. Abbreviations for agency database: ACEPD, Alachua County Environmental Protection Department; FGS, Florida Geological Survey; GSA, Geological Survey of Alabama; NWFWMD, Northwest Florida Water Management District; SFWMD, South Florida Water Management District; SRWMD, Suwannee River Water Management District; USGSALWSC, U.S. Geological Survey Alabama Water Science Center; USGS-FLWSC, U.S. Geological Survey Florida Water Science Center; GAWSC, U.S. Geological Survey Georgia Water Science Center; SCWSC, U.S. Geological Survey South Carolina Water Science Center. Abbreviations for Station names: BLVD., boulevard; CO, county; CR, county road; E, east; HS, high school; IFAS, Institute of Food and Agricultural Services; LK, lake; MW, monitoring well; N, north; NE, northeast; NE, northeast; NF, national forest; NW, northwest; PK, park; R., river; RD., road; SE, southeast; SE, southeast; SR, state road; ST., street; TWR, tower; UFA, Upper Floridan aquifer; —, not available; \#, number; “, inches]

\begin{tabular}{|c|c|c|c|c|c|c|c|c|c|c|}
\hline State & $\begin{array}{c}\text { Agency } \\
\text { database }\end{array}$ & Station name & $\begin{array}{c}\text { USGS site } \\
\text { identification } \\
\text { number }\end{array}$ & $\begin{array}{l}\text { Local agency } \\
\text { unique site } \\
\text { identifier }\end{array}$ & County & $\begin{array}{l}\text { Latitude } \\
\text { (degree } \\
\text { minutes } \\
\text { and } \\
\text { seconds) } \\
\text { [DDMMSS] }\end{array}$ & $\begin{array}{l}\text { Longitude } \\
\text { (degree } \\
\text { minutes } \\
\text { and } \\
\text { seconds) } \\
\text { ] [DDMMSS] }\end{array}$ & $\begin{array}{c}\text { Date of } \\
\text { measure- } \\
\text { ment }\end{array}$ & $\begin{array}{c}\text { Water } \\
\text { level } \\
\text { altitude } \\
\text { (feet } \\
\text { above } \\
\text { NGVD 29) }\end{array}$ & Remarks \\
\hline GA & USGS-GAWSC & 21P002 & 315614083042901 & - & Telfair & 315615 & 830429 & $5 / 28 / 2010$ & 156 & \\
\hline GA & USGS-GAWSC & 21Q008 & 320535083013301 & - & Dodge & 320535 & 830133 & $5 / 27 / 2010$ & 176 & \\
\hline GA & USGS-GAWSC & $21 \mathrm{~S} 008$ & 322001083021501 & - & Laurens & 322001 & 830215 & $5 / 19 / 2010$ & 222 & \\
\hline GA & USGS-GAWSC & $21 \mathrm{~T} 001$ & 322652083033001 & - & Laurens & 322707 & 830328 & $5 / 23 / 2010$ & 228 & \\
\hline GA & USGS-GAWSC & 21U006 & 323243083034301 & - & Laurens & 323244 & 830343 & $5 / 2 / 2010$ & 210 & \\
\hline GA & USGS-GAWSC & $22 \mathrm{H} 003$ & 310751082553302 & - & Clinch & 310751 & 825533 & $5 / 25 / 2010$ & 57 & \\
\hline GA & USGS-GAWSC & $22 \mathrm{M} 003$ & 313903082590501 & - & Coffee & 313856 & 825860 & $5 / 26 / 2010$ & 134 & \\
\hline GA & USGS-GAWSC & $22 \mathrm{M} 009$ & 314053082533001 & - & Coffee & 314053 & 825330 & $5 / 26 / 2010$ & 91 & \\
\hline GA & USGS-GAWSC & $22 \mathrm{M} 010$ & 314200082550001 & - & Coffee & 314204 & 825507 & $5 / 26 / 2010$ & 105 & \\
\hline GA & USGS-GAWSC & $22 \mathrm{~N} 001$ & 314858082573901 & - & Telfair & 314856 & 825743 & $5 / 27 / 2010$ & 154 & \\
\hline GA & USGS-GAWSC & 22Q004 & 320545082531701 & - & Wheeler & 320543 & 825309 & $5 / 18 / 2010$ & 168 & \\
\hline GA & USGS-GAWSC & $22 \mathrm{~S} 024$ & 321616082573901 & - & Laurens & 321616 & 825739 & $5 / 19 / 2010$ & 205 & \\
\hline GA & USGS-GAWSC & $22 \mathrm{~T} 001$ & 322255082594501 & - & Laurens & 322256 & 825933 & $5 / 18 / 2010$ & 221 & \\
\hline GA & USGS-GAWSC & $22 \mathrm{~V} 001$ & 324142082542701 & - & Laurens & 324142 & 825427 & $5 / 19 / 2010$ & 226 & \\
\hline GA & USGS-GAWSC & $23 \mathrm{G} 006$ & 310054082475601 & - & Clinch & 310054 & 824756 & $5 / 25 / 2010$ & 51 & \\
\hline GA & USGS-GAWSC & 23J006 & 311904082452701 & - & Atkinson & 311901 & 824544 & $5 / 25 / 2010$ & 49 & \\
\hline GA & USGS-GAWSC & 23J017 & 311751082492101 & - & Atkinson & 311751 & 824921 & $5 / 25 / 2010$ & 50 & \\
\hline GA & USGS-GAWSC & 23L007 & 313019082505201 & - & Coffee & 313020 & 825052 & $5 / 24 / 2010$ & 49 & \\
\hline GA & USGS-GAWSC & 23L021 & 313325082462801 & - & Coffee & 313325 & 824628 & $5 / 25 / 2010$ & 48 & \\
\hline GA & USGS-GAWSC & 23N001 & 314522082481401 & - & Jeff Davis & 314536 & 824803 & $5 / 26 / 2010$ & 88 & \\
\hline GA & USGS-GAWSC & 23P003 & 315350082484701 & - & Telfair & 315350 & 824847 & $6 / 3 / 2010$ & 140 & \\
\hline GA & USGS-GAWSC & 23U008 & 323312082522501 & - & Laurens & 323316 & 825212 & $5 / 19 / 2010$ & 221 & \\
\hline GA & USGS-GAWSC & $23 \times 035$ & 325614082484301 & - & Washington & 325614 & 824845 & $5 / 28 / 2010$ & 421 & \\
\hline GA & USGS-GAWSC & 24E003 & 305015082395701 & - & Clinch & 305015 & 823957 & $5 / 25 / 2010$ & 49 & \\
\hline
\end{tabular}


Table 1. Water-level measurements in Florida and parts of Georgia, South Carolina, and Alabama, May-June 2010.-Continued

[NGVD 29, National Geodetic Vertical Datum of 1929. Abbreviations for agency database: ACEPD, Alachua County Environmental Protection Department; FGS, Florida Geological Survey; GSA, Geological Survey of Alabama; NWFWMD, Northwest Florida Water Management District; SFWMD, South Florida Water Management District; SRWMD, Suwannee River Water Management District; USGSALWSC, U.S. Geological Survey Alabama Water Science Center; USGS-FLWSC, U.S. Geological Survey Florida Water Science Center; GAWSC, U.S. Geological Survey Georgia Water Science Center; SCWSC, U.S. Geological Survey South Carolina Water Science Center. Abbreviations for Station names: BLVD., boulevard; CO, county; CR, county road; E, east; HS, high school; IFAS, Institute of Food and Agricultural Services; LK, lake; MW, monitoring well; N, north; NE, northeast; NE, northeast; NF, national forest; NW, northwest; PK, park; R., river; RD., road; SE, southeast; SE, southeast; SR, state road; ST., street; TWR, tower; UFA, Upper Floridan aquifer; - - not available; \#, number; “, inches]

\begin{tabular}{|c|c|c|c|c|c|c|c|c|c|c|}
\hline State & $\begin{array}{c}\text { Agency } \\
\text { database }\end{array}$ & Station name & $\begin{array}{c}\text { USGS site } \\
\text { identification } \\
\text { number }\end{array}$ & $\begin{array}{l}\text { Local agency } \\
\text { unique site } \\
\text { identifier }\end{array}$ & County & $\begin{array}{l}\text { Latitude } \\
\text { (degree } \\
\text { minutes } \\
\text { and } \\
\text { seconds) } \\
\text { [DDMMSS] }\end{array}$ & $\begin{array}{l}\text { Longitude } \\
\text { (degree } \\
\text { minutes } \\
\text { and } \\
\text { seconds) } \\
\text { [DDMMSS] }\end{array}$ & $\begin{array}{c}\text { Date of } \\
\text { measure- } \\
\text { ment }\end{array}$ & $\begin{array}{c}\text { Water } \\
\text { level } \\
\text { altitude } \\
\text { (feet } \\
\text { above } \\
\text { NGVD 29) }\end{array}$ & Remarks \\
\hline GA & USGS-GAWSC & $24 \mathrm{G} 005$ & 310058082423601 & - & Clinch & 310058 & 824236 & $5 / 25 / 2010$ & 50 & \\
\hline GA & USGS-GAWSC & $24 \mathrm{G} 006$ & 310254082394201 & - & Clinch & 310254 & 823942 & $5 / 25 / 2010$ & 52 & \\
\hline GA & USGS-GAWSC & 24K001 & 312612082423901 & - & Coffee & 312612 & 824239 & $5 / 24 / 2010$ & 50 & \\
\hline GA & USGS-GAWSC & 24L004 & 313632082412201 & - & Coffee & 313633 & 824122 & $5 / 25 / 2010$ & 55 & \\
\hline GA & USGS-GAWSC & $24 \mathrm{~N} 004$ & 314841082393101 & - & Jeff Davis & 314841 & 823931 & $5 / 26 / 2010$ & 41 & \\
\hline GA & USGS-GAWSC & 24P006 & 315545082410301 & - & Telfair & 315549 & 824105 & $5 / 27 / 2010$ & 129 & \\
\hline GA & USGS-GAWSC & $24 \mathrm{R} 003$ & 320753082422901 & - & Wheeler & 320753 & 824229 & $5 / 27 / 2010$ & 137 & \\
\hline GA & USGS-GAWSC & 25J002 & 312126082362501 & - & Ware & 312126 & 823625 & $5 / 27 / 2010$ & 33 & \\
\hline GA & USGS-GAWSC & $25 \mathrm{~L} 007$ & 313018082320901 & - & Bacon & 313018 & 823209 & $5 / 18 / 2010$ & 45 & \\
\hline GA & USGS-GAWSC & $25 \mathrm{M} 001$ & 314302082371101 & - & Jeff Davis & 314302 & 823711 & $5 / 26 / 2010$ & 54 & \\
\hline GA & USGS-GAWSC & $25 \mathrm{M} 002$ & 313854082353501 & - & Bacon & 313854 & 823535 & $5 / 18 / 2010$ & 52 & \\
\hline GA & USGS-GAWSC & $25 \mathrm{P} 001$ & 315321082345701 & - & Jeff Davis & 315319 & 823504 & $5 / 26 / 2010$ & 58 & \\
\hline GA & USGS-GAWSC & 25Q001 & 320226082301101 & - & Montgomery & 320226 & 823004 & $5 / 23 / 2010$ & 101 & \\
\hline GA & USGS-GAWSC & 25Q003 & 320037082321401 & - & Montgomery & 320038 & 823213 & $5 / 17 / 2010$ & 109 & \\
\hline GA & USGS-GAWSC & $25 \mathrm{~S} 001$ & 321653082320301 & - & Montgomery & 321654 & 823202 & $5 / 17 / 2010$ & 118 & \\
\hline GA & USGS-GAWSC & $25 \mathrm{~S} 002$ & 321711082353501 & - & Montgomery & 321721 & 823530 & $5 / 17 / 2010$ & 115 & \\
\hline GA & USGS-GAWSC & $25 \mathrm{~S} 008$ & 321916082320401 & - & Montgomery & 321916 & 823204 & $5 / 17 / 2010$ & 121 & \\
\hline GA & USGS-GAWSC & $25 \mathrm{U} 003$ & 323145082353201 & - & Emanuel & 323151 & 823527 & $5 / 21 / 2010$ & 198 & \\
\hline GA & USGS-GAWSC & $25 \mathrm{~V} 001$ & 324123082310301 & - & Johnson & 324124 & 823057 & $5 / 19 / 2010$ & 237 & \\
\hline GA & USGS-GAWSC & $25 \mathrm{X} 015$ & 325543082353801 & - & Washington & 325544 & 823537 & $5 / 28 / 2010$ & 331 & \\
\hline GA & USGS-GAWSC & 26L004 & 313242082291601 & - & Bacon & 313240 & 822902 & $5 / 27 / 2010$ & 29 & \\
\hline GA & USGS-GAWSC & 26L011 & 313103082242001 & - & Bacon & 313103 & 822420 & $5 / 27 / 2010$ & 46 & \\
\hline GA & USGS-GAWSC & $26 \mathrm{P} 007$ & 315950082280101 & - & Toombs & 315950 & 822801 & $5 / 17 / 2010$ & 71 & \\
\hline GA & USGS-GAWSC & 26Q002 & 320050082241501 & - & Toombs & 320051 & 822414 & $5 / 17 / 2010$ & 58 & \\
\hline
\end{tabular}


Table 1. Water-level measurements in Florida and parts of Georgia, South Carolina, and Alabama, May-June 2010.—Continued

[NGVD 29, National Geodetic Vertical Datum of 1929. Abbreviations for agency database: ACEPD, Alachua County Environmental Protection Department; FGS, Florida Geological Survey; GSA, Geological Survey of Alabama; NWFWMD, Northwest Florida Water Management District; SFWMD, South Florida Water Management District; SRWMD, Suwannee River Water Management District; USGSALWSC, U.S. Geological Survey Alabama Water Science Center; USGS-FLWSC, U.S. Geological Survey Florida Water Science Center; GAWSC, U.S. Geological Survey Georgia Water Science Center; SCWSC, U.S. Geological Survey South Carolina Water Science Center. Abbreviations for Station names: BLVD., boulevard; CO, county; CR, county road; E, east; HS, high school; IFAS, Institute of Food and Agricultural Services; LK, lake; MW, monitoring well; N, north; NE, northeast; NE, northeast; NF, national forest; NW, northwest; PK, park; R., river; RD., road; SE, southeast; SE, southeast; SR, state road; ST., street; TWR, tower; UFA, Upper Floridan aquifer; - , not available; \#, number; “, inches]

\begin{tabular}{|c|c|c|c|c|c|c|c|c|c|c|}
\hline State & $\begin{array}{c}\text { Agency } \\
\text { database }\end{array}$ & Station name & $\begin{array}{c}\text { USGS site } \\
\text { identification } \\
\text { number }\end{array}$ & $\begin{array}{l}\text { Local agency } \\
\text { unique site } \\
\text { identifier }\end{array}$ & County & $\begin{array}{l}\text { Latitude } \\
\text { (degree } \\
\text { minutes } \\
\text { and } \\
\text { seconds) } \\
\text { [DDMMSS] }\end{array}$ & $\begin{array}{l}\text { Longitude } \\
\text { (degree } \\
\text { minutes } \\
\text { and } \\
\text { seconds) } \\
\text { [DDMMSS] }\end{array}$ & $\begin{array}{c}\text { Date of } \\
\text { measure- } \\
\text { ment }\end{array}$ & $\begin{array}{c}\text { Water } \\
\text { level } \\
\text { altitude } \\
\text { (feet } \\
\text { above } \\
\text { NGVD 29) }\end{array}$ & Remarks \\
\hline GA & USGS-GAWSC & 26R001 & 321302082243601 & - & Toombs & 321303 & 822435 & $5 / 23 / 2010$ & 113 & \\
\hline GA & USGS-GAWSC & $26 \mathrm{~T} 001$ & 322703082274301 & - & Treutlen & 322701 & 822743 & $5 / 18 / 2010$ & 174 & \\
\hline GA & USGS-GAWSC & $26 \times 005$ & 325454082271301 & - & Jefferson & 325458 & 822707 & $5 / 27 / 2010$ & 281 & \\
\hline GA & USGS-GAWSC & 27E004 & 304942082213801 & - & Charlton & 304944 & 822137 & $5 / 23 / 2010$ & 48 & \\
\hline GA & USGS-GAWSC & $27 \mathrm{G} 003$ & 310706082155101 & - & Ware & 310707 & 821555 & $5 / 23 / 2010$ & 49 & \\
\hline GA & USGS-GAWSC & $27 \mathrm{G} 006$ & 310320082161801 & - & Ware & 310321 & 821617 & $5 / 26 / 2010$ & 48 & \\
\hline GA & USGS-GAWSC & $27 \mathrm{H} 001$ & 311242082213901 & - & Ware & 311243 & 822127 & $5 / 17 / 2010$ & 44 & \\
\hline GA & USGS-GAWSC & 27L001 & 313538082162001 & - & Irwin & 313538 & 831620 & $5 / 25 / 2010$ & 193 & \\
\hline GA & USGS-GAWSC & 27L002 & 313223082171201 & - & Bacon & 313223 & 821712 & $5 / 24 / 2010$ & 41 & \\
\hline GA & USGS-GAWSC & 27M001 & 314028082220901 & - & Appling & 314036 & 822202 & $5 / 18 / 2010$ & 43 & \\
\hline GA & USGS-GAWSC & 27N006 & 314613082194801 & - & Appling & 314614 & 821947 & $5 / 18 / 2010$ & 43 & \\
\hline GA & USGS-GAWSC & 27N012 & 314725082152201 & - & Appling & 314725 & 821522 & $5 / 18 / 2010$ & 46 & \\
\hline GA & USGS-GAWSC & 27P004 & 315604082202201 & - & Appling & 315605 & 822021 & $5 / 20 / 2010$ & 61 & \\
\hline GA & USGS-GAWSC & 27Q002 & 320436082185801 & - & Toombs & 320437 & 821857 & $5 / 18 / 2010$ & 50 & \\
\hline GA & USGS-GAWSC & 27R004 & 320827082181101 & - & Toombs & 320829 & 821809 & $5 / 18 / 2010$ & 70 & \\
\hline GA & USGS-GAWSC & $27 \mathrm{~S} 002$ & 321807082182801 & - & Emanuel & 321801 & 821824 & $5 / 21 / 2010$ & 101 & \\
\hline GA & USGS-GAWSC & 27U005 & 323632082201601 & - & Emanuel & 323633 & 822015 & $5 / 20 / 2010$ & 184 & \\
\hline GA & USGS-GAWSC & 28D001 & 304256082092101 & - & Charlton & 304257 & 820920 & $5 / 26 / 2010$ & 43 & \\
\hline GA & USGS-GAWSC & 28J003 & 311820082145701 & - & Pierce & 311843 & 821456 & $5 / 26 / 2010$ & 48 & \\
\hline GA & USGS-GAWSC & $28 \mathrm{~K} 001$ & 312355082084201 & - & Pierce & 312348 & 820841 & $5 / 25 / 2010$ & 46 & \\
\hline GA & USGS-GAWSC & 28L007 & 313425082081201 & - & Appling & 313425 & 820812 & $5 / 24 / 2010$ & 42 & \\
\hline GA & USGS-GAWSC & 28R001 & 321110082131501 & - & Toombs & 321106 & 821313 & $5 / 20 / 2010$ & 63 & \\
\hline GA & USGS-GAWSC & $28 \mathrm{~S} 004$ & 321710082102601 & - & Tattnall & 321711 & 821025 & $5 / 20 / 2010$ & 86 & \\
\hline GA & USGS-GAWSC & 28T001 & 322630082125101 & - & Emanuel & 322631 & 821244 & $5 / 24 / 2010$ & 100 & \\
\hline
\end{tabular}


Table 1. Water-level measurements in Florida and parts of Georgia, South Carolina, and Alabama, May-June 2010.—Continued

[NGVD 29, National Geodetic Vertical Datum of 1929. Abbreviations for agency database: ACEPD, Alachua County Environmental Protection Department; FGS, Florida Geological Survey; GSA, Geological Survey of Alabama; NWFWMD, Northwest Florida Water Management District; SFWMD, South Florida Water Management District; SRWMD, Suwannee River Water Management District; USGSALWSC, U.S. Geological Survey Alabama Water Science Center; USGS-FLWSC, U.S. Geological Survey Florida Water Science Center; GAWSC, U.S. Geological Survey Georgia Water Science Center; SCWSC, U.S. Geological Survey South Carolina Water Science Center. Abbreviations for Station names: BLVD., boulevard; CO, county; CR, county road; E, east; HS, high school; IFAS, Institute of Food and Agricultural Services; LK, lake; MW, monitoring well; N, north; NE, northeast; NE, northeast; NF, national forest; NW, northwest; PK, park; R., river; RD., road; SE, southeast; SE, southeast; SR, state road; ST., street; TWR, tower; UFA, Upper Floridan aquifer; - - not available; \#, number; “, inches]

\begin{tabular}{|c|c|c|c|c|c|c|c|c|c|c|}
\hline State & $\begin{array}{c}\text { Agency } \\
\text { database }\end{array}$ & Station name & $\begin{array}{c}\text { USGS site } \\
\text { identification } \\
\text { number }\end{array}$ & $\begin{array}{l}\text { Local agency } \\
\text { unique site } \\
\text { identifier }\end{array}$ & County & $\begin{array}{l}\text { Latitude } \\
\text { (degree } \\
\text { minutes } \\
\text { and } \\
\text { seconds) } \\
\text { [DDMMSS] }\end{array}$ & $\begin{array}{l}\text { Longitude } \\
\text { (degree } \\
\text { minutes } \\
\text { and } \\
\text { seconds) } \\
\text { [DDMMSS] }\end{array}$ & $\begin{array}{c}\text { Date of } \\
\text { measure- } \\
\text { ment }\end{array}$ & $\begin{array}{c}\text { Water } \\
\text { level } \\
\text { altitude } \\
\text { (feet } \\
\text { above } \\
\text { NGVD 29) }\end{array}$ & Remarks \\
\hline GA & USGS-GAWSC & 28U002 & 323503082084901 & - & Emanuel & 323504 & 820848 & $5 / 20 / 2010$ & 139 & \\
\hline GA & USGS-GAWSC & 28U004 & 323056082095401 & - & Emanuel & 323057 & 820953 & $5 / 24 / 2010$ & 136 & \\
\hline GA & USGS-GAWSC & $28 \mathrm{~V} 001$ & 324115082125301 & - & Emanuel & 324116 & 821252 & $5 / 20 / 2010$ & 177 & \\
\hline GA & USGS-GAWSC & 28W002 & 324940082142601 & - & Burke & 324941 & 821425 & $5 / 26 / 2010$ & 209 & \\
\hline GA & USGS-GAWSC & $28 X 004$ & 325557082084501 & - & Burke & 325558 & 820844 & $5 / 26 / 2010$ & 208 & \\
\hline GA & USGS-GAWSC & $29 \mathrm{H} 004$ & 311116082002101 & - & Brantley & 311116 & 820021 & $5 / 26 / 2010$ & 39 & \\
\hline GA & USGS-GAWSC & 29M002 & 314026082071401 & - & Wayne & 314027 & 820713 & $5 / 19 / 2010$ & 45 & \\
\hline GA & USGS-GAWSC & $29 \mathrm{M} 005$ & 314026082014201 & - & Wayne & 313951 & 820141 & $5 / 19 / 2010$ & 38 & \\
\hline GA & USGS-GAWSC & $29 \mathrm{~N} 003$ & 314813082025201 & - & Wayne & 314825 & 820303 & $5 / 19 / 2010$ & 41 & \\
\hline GA & USGS-GAWSC & 29R001 & 321053082065101 & - & Tattnall & 321057 & 820647 & $5 / 20 / 2010$ & 37 & \\
\hline GA & USGS-GAWSC & 29R007 & 321322082015701 & - & Tattnall & 321322 & 820157 & $5 / 19 / 2010$ & 32 & \\
\hline GA & USGS-GAWSC & 29R008 & 320906082064101 & - & Tattnall & 320906 & 820641 & $5 / 19 / 2010$ & 29 & \\
\hline GA & USGS-GAWSC & 29T009 & 322958082023801 & - & Candler & 322948 & 820221 & $5 / 20 / 2010$ & 113 & \\
\hline GA & USGS-GAWSC & 29T010 & 322316082035701 & - & Candler & 322317 & 820356 & $5 / 20 / 2010$ & 114 & \\
\hline GA & USGS-GAWSC & 29V001 & 323858082051401 & - & Emanuel & 323855 & 820524 & $5 / 20 / 2010$ & 161 & \\
\hline GA & USGS-GAWSC & 30E008 & 304952081541201 & - & Camden & 304953 & 815411 & $5 / 17 / 2010$ & 38 & \\
\hline GA & USGS-GAWSC & $30 \mathrm{H} 005$ & 311233081573301 & - & Brantley & 311232 & 815734 & $5 / 26 / 2010$ & 38 & \\
\hline GA & USGS-GAWSC & 30L003 & 313701081543501 & - & Wayne & 313702 & 815433 & $5 / 23 / 2010$ & 21 & \\
\hline GA & USGS-GAWSC & 30L011 & 313041081594401 & - & Wayne & 313042 & 815943 & $5 / 19 / 2010$ & 31 & \\
\hline GA & USGS-GAWSC & 30R005 & 321032081535001 & - & Evans & 321035 & 815349 & $5 / 24 / 2010$ & 30 & \\
\hline GA & USGS-GAWSC & 30S001 & 322015081540601 & - & Bulloch & 322016 & 815405 & $5 / 19 / 2010$ & 51 & \\
\hline GA & USGS-GAWSC & 30U005 & 323216081560801 & - & Bulloch & 323217 & 815607 & $5 / 19 / 2010$ & 97 & \\
\hline GA & USGS-GAWSC & 30V002 & 323912081555501 & - & Jenkins & 323913 & 815558 & $5 / 26 / 2010$ & 145 & \\
\hline GA & USGS-GAWSC & 30W002 & 325227081571201 & - & Jenkins & 325228 & 815711 & $5 / 26 / 2010$ & 158 & \\
\hline
\end{tabular}


Table 1. Water-level measurements in Florida and parts of Georgia, South Carolina, and Alabama, May-June 2010.—Continued

[NGVD 29, National Geodetic Vertical Datum of 1929. Abbreviations for agency database: ACEPD, Alachua County Environmental Protection Department; FGS, Florida Geological Survey; GSA, Geological Survey of Alabama; NWFWMD, Northwest Florida Water Management District; SFWMD, South Florida Water Management District; SRWMD, Suwannee River Water Management District; USGSALWSC, U.S. Geological Survey Alabama Water Science Center; USGS-FLWSC, U.S. Geological Survey Florida Water Science Center; GAWSC, U.S. Geological Survey Georgia Water Science Center; SCWSC, U.S. Geological Survey South Carolina Water Science Center. Abbreviations for Station names: BLVD., boulevard; CO, county; CR, county road; E, east; HS, high school; IFAS, Institute of Food and Agricultural Services; LK, lake; MW, monitoring well; N, north; NE, northeast; NE, northeast; NF, national forest; NW, northwest; PK, park; R., river; RD., road; SE, southeast; SE, southeast; SR, state road; ST., street; TWR, tower; UFA, Upper Floridan aquifer; —, not available; \#, number; “, inches]

\begin{tabular}{|c|c|c|c|c|c|c|c|c|c|c|}
\hline State & $\begin{array}{c}\text { Agency } \\
\text { database }\end{array}$ & Station name & $\begin{array}{c}\text { USGS site } \\
\text { identification } \\
\text { number }\end{array}$ & $\begin{array}{l}\text { Local agency } \\
\text { unique site } \\
\text { identifier }\end{array}$ & County & $\begin{array}{l}\text { Latitude } \\
\text { (degree } \\
\text { minutes } \\
\text { and } \\
\text { seconds) } \\
\text { [DDMMSS] }\end{array}$ & $\begin{array}{l}\text { Longitude } \\
\text { (degree } \\
\text { minutes } \\
\text { and } \\
\text { seconds) } \\
\text { [DDMMSS] }\end{array}$ & $\begin{array}{c}\text { Date of } \\
\text { measure- } \\
\text { ment }\end{array}$ & $\begin{array}{c}\text { Water } \\
\text { level } \\
\text { altitude } \\
\text { (feet } \\
\text { above } \\
\text { NGVD 29) }\end{array}$ & Remarks \\
\hline GA & USGS-GAWSC & $30 \times 009$ & 325712081582201 & - & Burke & 325712 & 815821 & $5 / 26 / 2010$ & 239 & \\
\hline GA & USGS-GAWSC & 31E001 & 304830081481201 & - & Camden & 304831 & 814811 & $5 / 17 / 2010$ & 40 & \\
\hline GA & USGS-GAWSC & 31F022 & 305627081473101 & - & Camden & 305624 & 814834 & $5 / 17 / 2010$ & 41 & \\
\hline GA & USGS-GAWSC & $31 \mathrm{H} 005$ & 311214081512601 & - & Brantley & 311220 & 815118 & $5 / 26 / 2010$ & 33 & \\
\hline GA & USGS-GAWSC & 31M011 & 313924081501001 & - & Wayne & 313925 & 815009 & $5 / 18 / 2010$ & 0 & \\
\hline GA & USGS-GAWSC & $31 \mathrm{M} 013$ & 313859081495901 & - & Wayne & 313901 & 814958 & $5 / 19 / 2010$ & 6 & \\
\hline GA & USGS-GAWSC & $31 \mathrm{M} 014$ & 313911081502001 & - & Wayne & 313912 & 815019 & $5 / 18 / 2010$ & 1 & \\
\hline GA & USGS-GAWSC & $31 \mathrm{M} 016$ & 313953081502901 & - & Wayne & 313932 & 815050 & $5 / 19 / 2010$ & 4 & \\
\hline GA & USGS-GAWSC & $31 \mathrm{M} 031$ & 313838081493101 & - & Wayne & 313807 & 814938 & $5 / 20 / 2010$ & 12 & \\
\hline GA & USGS-GAWSC & $31 \mathrm{~T} 010$ & 322700081464401 & - & Bulloch & 322701 & 814645 & $5 / 19 / 2010$ & 51 & \\
\hline GA & USGS-GAWSC & 31U008 & 323123081511601 & - & Bulloch & 323124 & 815115 & $5 / 23 / 2010$ & 118 & \\
\hline GA & USGS-GAWSC & $32 \mathrm{E} 031$ & 304922081435501 & - & Camden & 304923 & 814354 & $5 / 17 / 2010$ & 40 & \\
\hline GA & USGS-GAWSC & 32E032 & 304809081404601 & - & Camden & 304808 & 814045 & $5 / 17 / 2010$ & 39 & \\
\hline GA & USGS-GAWSC & 32J003 & 311728081413301 & - & Glynn & 311729 & 814132 & $5 / 18 / 2010$ & 32 & \\
\hline GA & USGS-GAWSC & $32 \mathrm{~K} 014$ & 312555081403901 & - & Wayne & 312556 & 814038 & $5 / 18 / 2010$ & 24 & \\
\hline GA & USGS-GAWSC & 32L015 & 313253081433502 & - & Wayne & 313253 & 814335 & $5 / 23 / 2010$ & 17 & \\
\hline GA & USGS-GAWSC & $32 \mathrm{M} 002$ & 314241081444501 & - & Long & 314233 & 814433 & $5 / 19 / 2010$ & 8 & \\
\hline GA & USGS-GAWSC & $32 \mathrm{P} 007$ & 315753081384301 & - & Liberty & 315753 & 813843 & $5 / 20 / 2010$ & 2 & \\
\hline GA & USGS-GAWSC & $32 \mathrm{~V} 003$ & 324031081404701 & - & Screven & 324031 & 814046 & $5 / 18 / 2010$ & 97 & \\
\hline GA & USGS-GAWSC & 32W014 & 324508081384701 & - & Screven & 324511 & 813837 & $5 / 18 / 2010$ & 103 & \\
\hline GA & USGS-GAWSC & $32 \times 035$ & 325339081400001 & - & Screven & 325341 & 813958 & $5 / 18 / 2010$ & 130 & \\
\hline GA & USGS-GAWSC & $32 \times 040$ & 325649081410601 & - & Screven & 325648 & 814103 & $5 / 18 / 2010$ & 141 & \\
\hline GA & USGS-GAWSC & 33D069 & 304313081330001 & - & Camden & 304314 & 813259 & $5 / 23 / 2010$ & 27 & \\
\hline GA & USGS-GAWSC & 33E027 & 304756081311101 & - & Camden & 304757 & 813110 & $5 / 23 / 2010$ & 36 & \\
\hline
\end{tabular}


Table 1. Water-level measurements in Florida and parts of Georgia, South Carolina, and Alabama, May-June 2010.—Continued

[NGVD 29, National Geodetic Vertical Datum of 1929. Abbreviations for agency database: ACEPD, Alachua County Environmental Protection Department; FGS, Florida Geological Survey; GSA, Geological Survey of Alabama; NWFWMD, Northwest Florida Water Management District; SFWMD, South Florida Water Management District; SRWMD, Suwannee River Water Management District; USGSALWSC, U.S. Geological Survey Alabama Water Science Center; USGS-FLWSC, U.S. Geological Survey Florida Water Science Center; GAWSC, U.S. Geological Survey Georgia Water Science Center; SCWSC, U.S. Geological Survey South Carolina Water Science Center. Abbreviations for Station names: BLVD., boulevard; CO, county; CR, county road; E, east; HS, high school; IFAS, Institute of Food and Agricultural Services; LK, lake; MW, monitoring well; N, north; NE, northeast; NE, northeast; NF, national forest; NW, northwest; PK, park; R., river; RD., road; SE, southeast; SE, southeast; SR, state road; ST., street; TWR, tower; UFA, Upper Floridan aquifer; - - not available; \#, number; “, inches]

\begin{tabular}{|c|c|c|c|c|c|c|c|c|c|c|}
\hline State & $\begin{array}{c}\text { Agency } \\
\text { database }\end{array}$ & Station name & $\begin{array}{c}\text { USGS site } \\
\text { identification } \\
\text { number }\end{array}$ & $\begin{array}{l}\text { Local agency } \\
\text { unique site } \\
\text { identifier }\end{array}$ & County & $\begin{array}{l}\text { Latitude } \\
\text { (degree } \\
\text { minutes } \\
\text { and } \\
\text { seconds) } \\
\text { [DDMMS] }\end{array}$ & $\begin{array}{l}\text { Longitude } \\
\text { (degree } \\
\text { minutes } \\
\text { and } \\
\text { seconds) } \\
\text { ] [DDMMSS] }\end{array}$ & $\begin{array}{c}\text { Date of } \\
\text { measure- } \\
\text { ment }\end{array}$ & $\begin{array}{c}\text { Water } \\
\text { level } \\
\text { altitude } \\
\text { (feet } \\
\text { above } \\
\text { NGVD 29) }\end{array}$ & Remarks \\
\hline GA & USGS-GAWSC & 33G008 & 310707081320001 & - & Glynn & 310708 & 813157 & $5 / 18 / 2010$ & 26 & \\
\hline GA & USGS-GAWSC & $33 \mathrm{H} 133$ & 311007081301702 & - & Glynn & 311008 & 813016 & $5 / 23 / 2010$ & 4 & \\
\hline GA & USGS-GAWSC & $33 \mathrm{H} 141$ & 311045081323301 & - & Glynn & 311045 & 813230 & $5 / 18 / 2010$ & 14 & \\
\hline GA & USGS-GAWSC & $33 \mathrm{H} 177$ & 310740081361301 & - & Glynn & 310740 & 813613 & $5 / 18 / 2010$ & 31 & \\
\hline GA & USGS-GAWSC & $33 \mathrm{H} 193$ & 311345081370402 & - & Glynn & 311346 & 813703 & $5 / 18 / 2010$ & 26 & \\
\hline GA & USGS-GAWSC & $33 \mathrm{H} 324$ & 311022081304601 & - & Glynn & 311022 & 813046 & $5 / 23 / 2010$ & -4 & \\
\hline GA & USGS-GAWSC & 33J027 & 311958081352701 & - & Glynn & 312001 & 813534 & $5 / 19 / 2010$ & 21 & \\
\hline GA & USGS-GAWSC & 33L027 & 313028081354201 & - & Mcintosh & 313021 & 813543 & $5 / 20 / 2010$ & 16 & \\
\hline GA & USGS-GAWSC & 33M004 & 313845081361701 & - & Long & 313855 & 813603 & $5 / 23 / 2010$ & 9 & \\
\hline GA & USGS-GAWSC & $33 \mathrm{~N} 089$ & 314552081372101 & - & Liberty & 314553 & 813720 & $5 / 19 / 2010$ & 5 & \\
\hline GA & USGS-GAWSC & 33Р029 & 315434081364702 & - & Liberty & 315434 & 813647 & $5 / 19 / 2010$ & 8 & \\
\hline GA & USGS-GAWSC & 33U021 & 323525081301101 & - & Screven & 323519 & 813013 & $5 / 18 / 2010$ & 72 & \\
\hline GA & USGS-GAWSC & $33 \mathrm{U} 023$ & 323118081320801 & - & Screven & 323117 & 813203 & $6 / 1 / 2010$ & 79 & \\
\hline GA & USGS-GAWSC & $33 \times 027$ & 325504081300501 & - & Screven & 325505 & 813005 & $5 / 18 / 2010$ & 77 & \\
\hline GA & USGS-GAWSC & $34 \mathrm{G} 033$ & 310418081244701 & - & Glynn & 310423 & 812451 & $5 / 23 / 2010$ & 34 & \\
\hline GA & USGS-GAWSC & $34 \mathrm{H} 062$ & 311005081282701 & - & Glynn & 311006 & 812826 & $5 / 21 / 2010$ & 8 & \\
\hline GA & USGS-GAWSC & $34 \mathrm{H} 328$ & 311319081232901 & - & Glynn & 311320 & 812328 & $5 / 18 / 2010$ & 16 & \\
\hline GA & USGS-GAWSC & $34 \mathrm{H} 334$ & 310938081285301 & - & Glynn & 310938 & 812851 & $5 / 23 / 2010$ & 14 & \\
\hline GA & USGS-GAWSC & $34 \mathrm{H} 371$ & 310818081293701 & - & Glynn & 310819 & 812936 & $5 / 23 / 2010$ & 17 & \\
\hline GA & USGS-GAWSC & $34 \mathrm{H} 504$ & 310810081292801 & - & Glynn & 310811 & 812927 & $5 / 23 / 2010$ & 16 & \\
\hline GA & USGS-GAWSC & $34 \mathrm{H} 514$ & 310931081291002 & - & Glynn & 310931 & 812910 & $5 / 23 / 2010$ & 8 & \\
\hline GA & USGS-GAWSC & $34 \mathrm{~K} 073$ & 312507081281301 & - & Mcintosh & 312507 & 812815 & $5 / 20 / 2010$ & 9 & \\
\hline GA & USGS-GAWSC & $34 \mathrm{~K} 095$ & 312251081244301 & - & Mcintosh & 312255 & 812442 & $5 / 20 / 2010$ & 9 & \\
\hline GA & USGS-GAWSC & 34L048 & 313054081245501 & - & Mcintosh & 313055 & 812454 & $5 / 20 / 2010$ & 12 & \\
\hline
\end{tabular}


Table 1. Water-level measurements in Florida and parts of Georgia, South Carolina, and Alabama, May-June 2010.—Continued

[NGVD 29, National Geodetic Vertical Datum of 1929. Abbreviations for agency database: ACEPD, Alachua County Environmental Protection Department; FGS, Florida Geological Survey; GSA, Geological Survey of Alabama; NWFWMD, Northwest Florida Water Management District; SFWMD, South Florida Water Management District; SRWMD, Suwannee River Water Management District; USGSALWSC, U.S. Geological Survey Alabama Water Science Center; USGS-FLWSC, U.S. Geological Survey Florida Water Science Center; GAWSC, U.S. Geological Survey Georgia Water Science Center; SCWSC, U.S. Geological Survey South Carolina Water Science Center. Abbreviations for Station names: BLVD., boulevard; CO, county; CR, county road; E, east; HS, high school; IFAS, Institute of Food and Agricultural Services; LK, lake; MW, monitoring well; N, north; NE, northeast; NE, northeast; NF, national forest; NW, northwest; PK, park; R., river; RD., road; SE, southeast; SE, southeast; SR, state road; ST., street; TWR, tower; UFA, Upper Floridan aquifer; —, not available; \#, number; “, inches]

\begin{tabular}{|c|c|c|c|c|c|c|c|c|c|c|}
\hline State & $\begin{array}{c}\text { Agency } \\
\text { database }\end{array}$ & Station name & $\begin{array}{c}\text { USGS site } \\
\text { identification } \\
\text { number }\end{array}$ & $\begin{array}{l}\text { Local agency } \\
\text { unique site } \\
\text { identifier }\end{array}$ & County & $\begin{array}{l}\text { Latitude } \\
\text { (degree } \\
\text { minutes } \\
\text { and } \\
\text { seconds) } \\
\text { [DDMMS] }\end{array}$ & $\begin{array}{l}\text { Longitude } \\
\text { (degree } \\
\text { minutes } \\
\text { and } \\
\text { seconds) } \\
\text { ] [DDMMSS] }\end{array}$ & $\begin{array}{c}\text { Date of } \\
\text { measure- } \\
\text { ment }\end{array}$ & $\begin{array}{c}\text { Water } \\
\text { level } \\
\text { altitude } \\
\text { (feet } \\
\text { above } \\
\text { NGVD 29) }\end{array}$ & Remarks \\
\hline GA & USGS-GAWSC & $34 \mathrm{M} 075$ & 313901081234101 & - & Liberty & 313909 & 812343 & $5 / 20 / 2010$ & -3 & \\
\hline GA & USGS-GAWSC & 34N089 & 315214081235301 & - & Liberty & 315215 & 812352 & $5 / 23 / 2010$ & -7 & \\
\hline GA & USGS-GAWSC & 34R039 & 321145081260401 & - & Bryan & 321146 & 812602 & $5 / 19 / 2010$ & 2 & \\
\hline GA & USGS-GAWSC & $34 \mathrm{~S} 011$ & 321742081234904 & - & Effingham & 321743 & 812348 & $5 / 23 / 2010$ & 6 & \\
\hline GA & USGS-GAWSC & $35 \mathrm{H} 070$ & 311456081210503 & - & Glynn & 311454 & 812105 & $5 / 23 / 2010$ & 11 & \\
\hline GA & USGS-GAWSC & $35 \mathrm{M} 013$ & 313823081154201 & - & Mcintosh & 313824 & 811541 & $5 / 23 / 2010$ & -5 & \\
\hline GA & USGS-GAWSC & $35 \mathrm{P} 110$ & 315443081185902 & - & Bryan & 315443 & 811859 & $5 / 23 / 2010$ & -15 & \\
\hline GA & USGS-GAWSC & $35 \mathrm{R} 018$ & 320823081193301 & - & Chatham & 320824 & 811932 & $5 / 20 / 2010$ & -10 & \\
\hline GA & USGS-GAWSC & $35 \mathrm{R} 025$ & 320738081175501 & - & Chatham & 320739 & 811753 & $5 / 21 / 2010$ & -17 & \\
\hline GA & USGS-GAWSC & $35 \mathrm{~T} 003$ & 322236081191001 & - & Effingham & 322237 & 811909 & $5 / 23 / 2010$ & 4 & \\
\hline GA & USGS-GAWSC & $35 \mathrm{~T} 010$ & 322949081173201 & - & Effingham & 322949 & 811731 & $6 / 2 / 2010$ & 30 & \\
\hline GA & USGS-GAWSC & 36Q007 & 320714081090001 & - & Chatham & 320715 & 810859 & $5 / 21 / 2010$ & -68 & \\
\hline GA & USGS-GAWSC & 36Q008 & 320530081085001 & - & Chatham & 320531 & 810849 & $5 / 23 / 2010$ & -69 & \\
\hline GA & USGS-GAWSC & 36Q020 & 320021081124801 & - & Chatham & 320022 & 811247 & $5 / 23 / 2010$ & -31 & \\
\hline GA & USGS-GAWSC & 36Q283 & 320656081145801 & - & Chatham & 320657 & 811458 & $5 / 20 / 2010$ & -20 & \\
\hline GA & USGS-GAWSC & 36Q300 & 320536081121901 & - & Chatham & 320537 & 811218 & $5 / 21 / 2010$ & -50 & \\
\hline GA & USGS-GAWSC & 36R008 & 320920081095201 & - & Chatham & 320921 & 810951 & $5 / 21 / 2010$ & -42 & \\
\hline GA & USGS-GAWSC & $36 \mathrm{~S} 022$ & 321722081135601 & - & Effingham & 321723 & 811401 & $5 / 19 / 2010$ & -3 & \\
\hline GA & USGS-GAWSC & $36 \mathrm{~S} 034$ & 321644081140001 & - & Effingham & 321645 & 811400 & $5 / 19 / 2010$ & -16 & \\
\hline GA & USGS-GAWSC & $37 \mathrm{P} 114$ & 315906081011202 & - & Chatham & 315907 & 810111 & $5 / 23 / 2010$ & -35 & \\
\hline GA & USGS-GAWSC & 37Q016 & 320433081042701 & - & Chatham & 320434 & 810426 & $5 / 23 / 2010$ & -60 & \\
\hline GA & USGS-GAWSC & 37Q185 & 320622081063701 & - & Chatham & 320623 & 810636 & $5 / 23 / 2010$ & -79 & \\
\hline GA & USGS-GAWSC & 39Q003 & 320122080510204 & - & Chatham & 320123 & 805101 & $5 / 23 / 2010$ & -19 & \\
\hline GA & USGS-GAWSC & 39Q025 & 320127080511202 & - & Chatham & 320128 & 805111 & $5 / 23 / 2010$ & -22 & \\
\hline
\end{tabular}


Table 1. Water-level measurements in Florida and parts of Georgia, South Carolina, and Alabama, May-June 2010.—Continued

[NGVD 29, National Geodetic Vertical Datum of 1929. Abbreviations for agency database: ACEPD, Alachua County Environmental Protection Department; FGS, Florida Geological Survey; GSA, Geological Survey of Alabama; NWFWMD, Northwest Florida Water Management District; SFWMD, South Florida Water Management District; SRWMD, Suwannee River Water Management District; USGSALWSC, U.S. Geological Survey Alabama Water Science Center; USGS-FLWSC, U.S. Geological Survey Florida Water Science Center; GAWSC, U.S. Geological Survey Georgia Water Science Center; SCWSC, U.S. Geological Survey South Carolina Water Science Center. Abbreviations for Station names: BLVD., boulevard; CO, county; CR, county road; E, east; HS, high school; IFAS, Institute of Food and Agricultural Services; LK, lake; MW, monitoring well; N, north; NE, northeast; NE, northeast; NF, national forest; NW, northwest; PK, park; R., river; RD., road; SE, southeast; SE, southeast; SR, state road; ST., street; TWR, tower; UFA, Upper Floridan aquifer; - , not available; \#, number; “, inches]

\begin{tabular}{|c|c|c|c|c|c|c|c|c|c|c|}
\hline State & $\begin{array}{c}\text { Agency } \\
\text { database }\end{array}$ & Station name & $\begin{array}{c}\text { USGS site } \\
\text { identification } \\
\text { number }\end{array}$ & $\begin{array}{l}\text { Local agency } \\
\text { unique site } \\
\text { identifier }\end{array}$ & County & $\begin{array}{l}\text { Latitude } \\
\text { (degree } \\
\text { minutes } \\
\text { and } \\
\text { seconds) } \\
\text { [DDMMS] }\end{array}$ & $\begin{array}{l}\text { Longitude } \\
\text { (degree } \\
\text { minutes } \\
\text { and } \\
\text { seconds) } \\
\text { [DDMMSS] }\end{array}$ & $\begin{array}{c}\text { Date of } \\
\text { measure- } \\
\text { ment }\end{array}$ & $\begin{array}{c}\text { Water } \\
\text { level } \\
\text { altitude } \\
\text { (feet } \\
\text { above } \\
\text { NGVD 29) }\end{array}$ & Remarks \\
\hline $\mathrm{SC}$ & USGS-SCWSC & BFT-118 & 322518080445909 & - & Beaufort & 322519 & 804458 & $5 / 27 / 2010$ & -6 & \\
\hline $\mathrm{SC}$ & USGS-SCWSC & BFT-181 & 321818080405700 & - & Beaufort & 321824 & 804047 & $5 / 27 / 2010$ & 6 & \\
\hline $\mathrm{SC}$ & USGS-SCWSC & BFT-315 & 321558080431301 & - & Beaufort & 321546 & 804312 & $5 / 20 / 2010$ & 0 & \\
\hline $\mathrm{SC}$ & USGS-SCWSC & BFT-321 & 321218080411800 & - & Beaufort & 321219 & 804117 & $5 / 20 / 2010$ & -5 & \\
\hline $\mathrm{SC}$ & USGS-SCWSC & BFT-358 & 321454080494309 & - & Beaufort & 321455 & 804942 & $5 / 18 / 2010$ & -9 & \\
\hline $\mathrm{SC}$ & USGS-SCWSC & BFT-452 & 322353080261509 & - & Beaufort & 322354 & 802614 & $5 / 21 / 2010$ & 2 & \\
\hline $\mathrm{SC}$ & USGS-SCWSC & BFT-455 & 321953080280309 & - & Beaufort & 321954 & 802802 & $5 / 21 / 2010$ & 1 & \\
\hline $\mathrm{SC}$ & USGS-SCWSC & BFT-563 & 322228080325000 & - & Beaufort & 322229 & 803249 & $5 / 19 / 2010$ & 3 & \\
\hline $\mathrm{SC}$ & USGS-SCWSC & BFT-570 & 321805080581400 & - & Beaufort & 321806 & 805813 & $5 / 19 / 2010$ & -16 & \\
\hline $\mathrm{SC}$ & USGS-SCWSC & BFT-746 & 320722080484709 & - & Beaufort & 320723 & 804846 & $5 / 17 / 2010$ & -15 & \\
\hline $\mathrm{SC}$ & USGS-SCWSC & BFT-1311 & 323005080421108 & - & Beaufort & 323006 & 804210 & $5 / 28 / 2010$ & 4 & \\
\hline $\mathrm{SC}$ & USGS-SCWSC & BFT-1604 & 322611080282609 & - & Beaufort & 322612 & 802825 & $5 / 21 / 2010$ & -2 & \\
\hline $\mathrm{SC}$ & USGS-SCWSC & BFT-1609 & 322750080333300 & - & Beaufort & 322751 & 803332 & $5 / 21 / 2010$ & 1 & \\
\hline $\mathrm{SC}$ & USGS-SCWSC & BFT-1810 & 321603080432202 & - & Beaufort & 321604 & 804321 & $5 / 20 / 2010$ & 0 & \\
\hline $\mathrm{SC}$ & USGS-SCWSC & BFT-1970 & 322230080413600 & - & Beaufort & 322231 & 804135 & $5 / 27 / 2010$ & 6 & \\
\hline $\mathrm{SC}$ & USGS-SCWSC & BFT-2166 & 321533080455409 & - & Beaufort & 321534 & 804553 & $6 / 1 / 2010$ & -1 & \\
\hline $\mathrm{SC}$ & USGS-SCWSC & BFT-2187 & 321512080421909 & - & Beaufort & 321513 & 804218 & $6 / 1 / 2010$ & 0 & \\
\hline $\mathrm{SC}$ & USGS-SCWSC & BFT-2188 & 321524080430109 & - & Beaufort & 321525 & 804300 & $6 / 1 / 2010$ & 0 & \\
\hline $\mathrm{SC}$ & USGS-SCWSC & BFT-2196 & 321534080431409 & - & Beaufort & 321535 & 804313 & $6 / 1 / 2010$ & -1 & \\
\hline $\mathrm{SC}$ & USGS-SCWSC & BFT-2245 & 320846080501709 & - & Beaufort & 320847 & 805016 & $5 / 26 / 2010$ & -15 & \\
\hline $\mathrm{SC}$ & USGS-SCWSC & BFT-2247 & 320518080522008 & - & Beaufort & 320519 & 805219 & $5 / 26 / 2010$ & -23 & \\
\hline $\mathrm{SC}$ & USGS-SCWSC & BFT-2302 & 321815080474009 & - & Beaufort & 321816 & 804739 & $6 / 7 / 2010$ & -1 & \\
\hline $\mathrm{SC}$ & USGS-SCWSC & BFT-2303 & 321418080483109 & - & Beaufort & 321419 & 804830 & $6 / 7 / 2010$ & -5 & \\
\hline $\mathrm{SC}$ & USGS-SCWSC & BFT-2306 & 321350080502509 & - & Beaufort & 321351 & 805024 & $6 / 7 / 2010$ & -9 & \\
\hline
\end{tabular}


Table 1. Water-level measurements in Florida and parts of Georgia, South Carolina, and Alabama, May-June 2010.—Continued

[NGVD 29, National Geodetic Vertical Datum of 1929. Abbreviations for agency database: ACEPD, Alachua County Environmental Protection Department; FGS, Florida Geological Survey; GSA, Geological Survey of Alabama; NWFWMD, Northwest Florida Water Management District; SFWMD, South Florida Water Management District; SRWMD, Suwannee River Water Management District; USGSALWSC, U.S. Geological Survey Alabama Water Science Center; USGS-FLWSC, U.S. Geological Survey Florida Water Science Center; GAWSC, U.S. Geological Survey Georgia Water Science Center; SCWSC, U.S. Geological Survey South Carolina Water Science Center. Abbreviations for Station names: BLVD., boulevard; CO, county; CR, county road; E, east; HS, high school; IFAS, Institute of Food and Agricultural Services; LK, lake; MW, monitoring well; N, north; NE, northeast; NE, northeast; NF, national forest; NW, northwest; PK, park; R., river; RD., road; SE, southeast; SE, southeast; SR, state road; ST., street; TWR, tower; UFA, Upper Floridan aquifer; —, not available; \#, number; “, inches]

\begin{tabular}{|c|c|c|c|c|c|c|c|c|c|c|}
\hline State & $\begin{array}{c}\text { Agency } \\
\text { database }\end{array}$ & Station name & $\begin{array}{c}\text { USGS site } \\
\text { identification } \\
\text { number }\end{array}$ & $\begin{array}{l}\text { Local agency } \\
\text { unique site } \\
\text { identifier }\end{array}$ & County & $\begin{array}{l}\text { Latitude } \\
\text { (degree } \\
\text { minutes } \\
\text { and } \\
\text { seconds) } \\
\text { [DDMMS] }\end{array}$ & $\begin{array}{l}\text { Longitude } \\
\text { (degree } \\
\text { minutes } \\
\text { and } \\
\text { seconds) } \\
\text { ] [DDMMSS] }\end{array}$ & $\begin{array}{c}\text { Date of } \\
\text { measure- } \\
\text { ment }\end{array}$ & $\begin{array}{c}\text { Water } \\
\text { level } \\
\text { altitude } \\
\text { (feet } \\
\text { above } \\
\text { NGVD 29) }\end{array}$ & Remarks \\
\hline $\mathrm{SC}$ & USGS-SCWSC & BFT-2307 & 321329080494509 & - & Beaufort & 321330 & 804944 & $6 / 7 / 2010$ & -7 & \\
\hline $\mathrm{SC}$ & USGS-SCWSC & BFT-2313 & 321629080452509 & - & Beaufort & 321630 & 804524 & $6 / 1 / 2010$ & 1 & \\
\hline $\mathrm{SC}$ & USGS-SCWSC & BFT-2356 & 321211080524909 & - & Beaufort & 321212 & 805248 & $5 / 19 / 2010$ & -14 & \\
\hline $\mathrm{SC}$ & USGS-SCWSC & BFT-2404 & 322940080443000 & - & Beaufort & 322941 & 804429 & $5 / 19 / 2010$ & 19 & \\
\hline $\mathrm{SC}$ & USGS-SCWSC & HAM-50 & 324048081112009 & - & Hampton & 324049 & 811119 & $5 / 18 / 2010$ & 89 & \\
\hline $\mathrm{SC}$ & USGS-SCWSC & HAM-72 & 325841081064609 & - & Hampton & 325842 & 810645 & $5 / 25 / 2010$ & 102 & \\
\hline $\mathrm{SC}$ & USGS-SCWSC & HAM-73 & 325355081000800 & - & Hampton & 325306 & 810010 & $5 / 28 / 2010$ & 52 & \\
\hline $\mathrm{SC}$ & USGS-SCWSC & HAM-74 & 325242081022409 & - & Hampton & 325243 & 810223 & $5 / 28 / 2010$ & 73 & \\
\hline $\mathrm{SC}$ & USGS-SCWSC & HAM-76 & 324821080543500 & - & Hampton & 324822 & 805434 & $5 / 28 / 2010$ & 34 & \\
\hline $\mathrm{SC}$ & USGS-SCWSC & HAM-77 & 324327080524800 & - & Hampton & 324328 & 805247 & $5 / 17 / 2010$ & 16 & \\
\hline $\mathrm{SC}$ & USGS-SCWSC & HAM-83 & 324143080505900 & - & Hampton & 324153 & 805103 & $5 / 17 / 2010$ & 8 & \\
\hline $\mathrm{SC}$ & USGS-SCWSC & HAM-122 & 323949081193001 & - & Hampton & 323950 & 811929 & $5 / 18 / 2010$ & 89 & \\
\hline $\mathrm{SC}$ & USGS-SCWSC & HAM-174 & 324924081165909 & - & Hampton & 324925 & 811658 & $6 / 4 / 2010$ & 106 & \\
\hline $\mathrm{SC}$ & USGS-SCWSC & HAM-175 & 325530081025809 & - & Hampton & 325531 & 810257 & $6 / 2 / 2010$ & 67 & \\
\hline $\mathrm{SC}$ & USGS-SCWSC & НАM-314 & 324948081095700 & - & Hampton & 324949 & 810957 & $5 / 12 / 2010$ & 85 & \\
\hline $\mathrm{SC}$ & USGS-SCWSC & JAS-397 & 323134080520500 & - & Jasper & 323135 & 805204 & $5 / 17 / 2010$ & 2 & \\
\hline $\mathrm{SC}$ & USGS-SCWSC & JAS-402 & 322749081062700 & - & Jasper & 322750 & 810626 & $5 / 17 / 2010$ & 9 & \\
\hline $\mathrm{SC}$ & USGS-SCWSC & JAS-406 & 323536081033100 & - & Jasper & 323537 & 810330 & $5 / 19 / 2010$ & 59 & \\
\hline $\mathrm{SC}$ & USGS-SCWSC & JAS-425 & 323704080594509 & - & Jasper & 323705 & 805944 & $6 / 2 / 2010$ & 12 & \\
\hline $\mathrm{SC}$ & USGS-SCWSC & JAS-491 & 322853080582200 & - & Jasper & 322853 & 805822 & $5 / 12 / 2010$ & 1 & \\
\hline $\mathrm{SC}$ & USGS-SCWSC & JAS-0499 & 321022081043400 & - & Jasper & 321022 & 810434 & $5 / 18 / 2010$ & -44 & \\
\hline
\end{tabular}




\section{Prepared by:}

USGS Science Publishing Network

Raleigh Publishing Service Center

3916 Sunset Ridge Road

Raleigh, NC 27607

USGS Publishing Service Center staff:

Edited by Teresa L. Embry

Layout by James A. Tomberlin

For additional information regarding this publication, contact:

Director

USGS Florida Water Science Center

10500 University Center Drive, Suite 215

Tampa, FL 33612

email: GS-W-FL_center_director@usgs.gov

Or visit USGS Florida Water Science Center at:

http://fl.water.usgs.gov/ 

